Florida International University FIU Digital Commons

10-16-2015

\title{
Heat-Driven Self-Cooling System Based On Thermoelectric Generation Effect
}

Robel Kiflemariam

rkifl001@fiu.edu

DOI: $10.25148 /$ etd.FIDC000179

Follow this and additional works at: https://digitalcommons.fiu.edu/etd

Part of the Energy Systems Commons

\section{Recommended Citation}

Kiflemariam, Robel, "Heat-Driven Self-Cooling System Based On Thermoelectric Generation Effect" (2015). FIU Electronic Theses and Dissertations. 2281.

https://digitalcommons.fiu.edu/etd/2281

This work is brought to you for free and open access by the University Graduate School at FIU Digital Commons. It has been accepted for inclusion in FIU Electronic Theses and Dissertations by an authorized administrator of FIU Digital Commons. For more information, please contact dcc@fiu.edu. 


\title{
FLORIDA INTERNATIONAL UNIVERSITY
}

Miami, Florida

\section{HEAT-DRIVEN SELF-COOLING SYSTEM BASED ON THERMOELECTRIC GENERATION EFFECT}

\author{
A dissertation submitted in partial fulfillment of \\ the requirements for the degree of \\ DOCTOR OF PHILOSOPHY \\ in \\ MECHANICAL ENGINEERING \\ by
}

Robel Kiflemariam

2015 
To: Interim Dean Ranu Jung

College of Engineering and Computing

This dissertation, written by Robel Kiflemariam, and entitled, Heat-Driven SelfCooling System Based on Thermoelectric Generation Effect having been approved in respect to style and intellectual content, is referred to you for judgment.

We have read this thesis and recommend that it be approved.

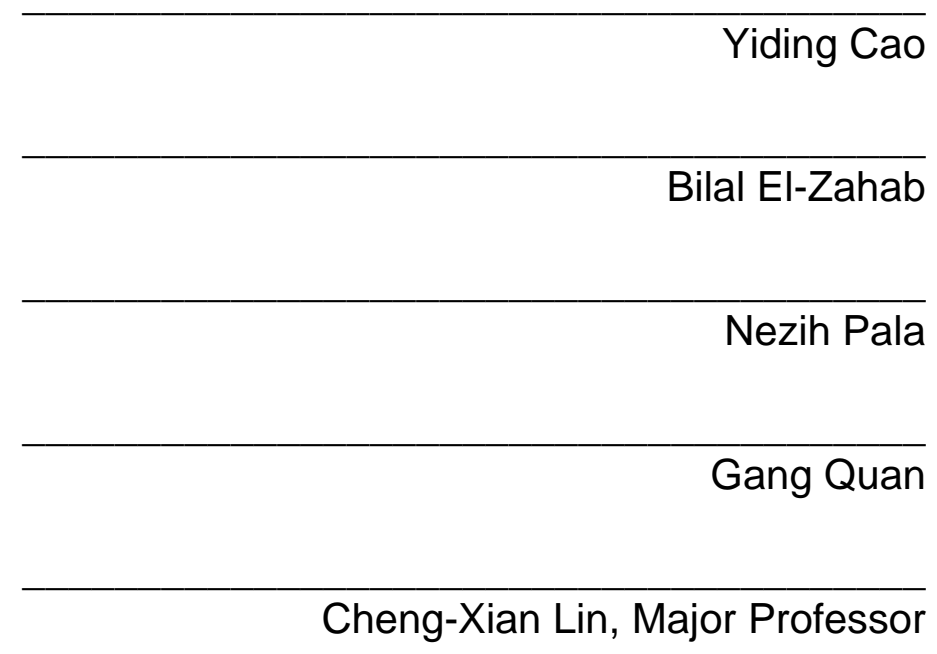

Date of Defense: October 16, 2015

The dissertation of Robel Kiflemariam is approved.

Interim Dean Ranu Jung College of Engineering and Computing

Dean Lakshmi N. Reddi University Graduate School

Florida International University, 2015 
(C) Copyright 2015 by Robel Kiflemariam

All rights reserved. 


\section{DEDICATION}

I dedicate my thesis to my family, my dad, mom and my sister and brothers who have always been to my side from the beginning to the end. 


\section{ACKNOWLEDGMENTS}

First I would like to thank my advisor Prof. Cheng-Xian Lin for his continuous support of my PhD study and invaluable advice and discussions. My thesis committee has given me guidance thought my research. I would like to thank Prof. Yiding Cao for his support and insightful advices. My sincere thanks also goes to Prof. Bilal El-Zahab for his important and insightful comments. I would also like to thank Prof. Nezih Pala and Prof Gang Quan for valuable comments and suggestions. I gratefully acknowledge my research group members and friends in Computational lab: Long Phan, Soheil Soleimanikutanaei, Esmail Ghasemi, Mack Telusma for their encouragement and assistance. 


\title{
ABSTRACT OF THE DISSERTATION \\ HEAT-DRIVEN SELF-COOLING SYSTEM BASED ON THERMOELECTRIC GENERATION EFFECT
}

\author{
by
}

Robel Kiflemariam

Florida International University, 2015

Miami, Florida

Professor Cheng-Xian Lin, Major Professor

This research entails the first comprehensive and systematic study on a heatdriven, self-cooling application based on the thermoelectric generation effect. The system was studied using the first and second laws of thermodynamics to provide a solid and basic understanding of the physical principles governing the system. Multiphysics equations that relate heat transfer, fluid dynamics and thermoelectric generation are derived. The equations are developed with increasing complexity, from the basic Carnot heat engine to externally and internally irreversible engines. A computational algorithm to systematically use the fundamental equations has been presented and computer code is implemented based on the algorithm.

Experiments were conducted to analyze the geometric and system parameters affecting the application of thermoelectric based self-cooling in devices. Experimental results show that for the highest heat input studied, the temperature of the device has been reduced by $20-40 \%$ as compared to the natural convection case. In addition, it has been found that in the self-cooling 
cases studied, convection thermal resistance could account for up to $60 \%$ of the total thermal resistance.

A general numerical methodology was developed to predict steady as well as transient thermal and electrical behavior of a thermoelectric generation-based self-cooling system. The methodology is implemented by using equation modeling capabilities to capture the thermo-electric coupled interaction in TEG elements, enabling the simulation of major heating effects as well as temperature and spatial dependent properties. An alternative methodology was also presented, which integrates specialized ANSI-C code to integrate thermoelectric effects, temperature-dependent properties and transient boundary conditions. It has been shown that the computational model is able to predict the experimental data with good accuracy (within 5\% error). A parametric study has been done using the model to study the effect of heat sink geometry on device temperature and power produced by TEG arrays.

In addition, a dynamic model suited for integration in control systems is developed. Therefore, the study has shown the potential for a heat driven selfcooling system and provides a comprehensive set of tools for analysis and design of thermoelectric generation. 


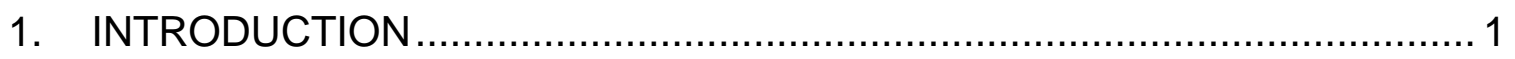

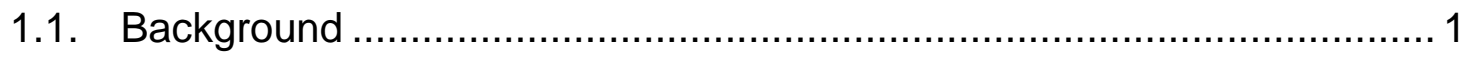

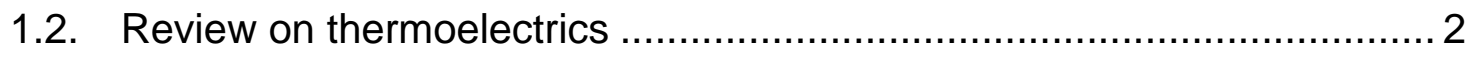

1.3. Review on Waste Heat Scavenging …........................................ 10

1.4. Review on Thermoelectric Generator-Based Self-cooling Applications. 17

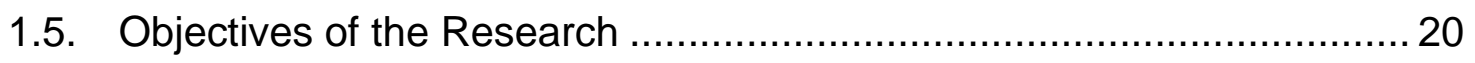

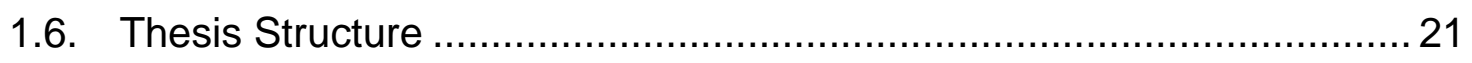

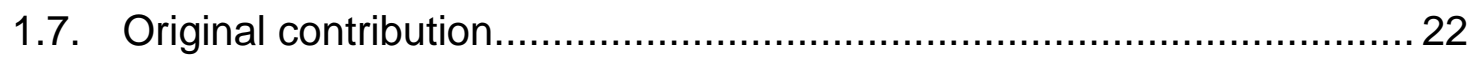

2. THERMODYNAMIC ANALYSIS OF SELF-COOLING SYSTEM ................ 24

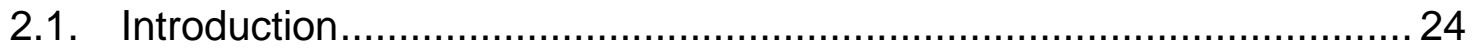

2.2. Thermoelectric couple governing equations …................................ 31

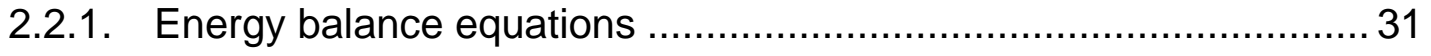

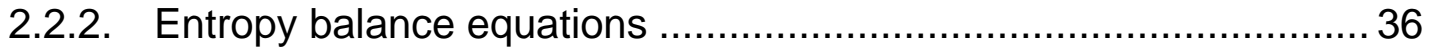

2.2.3. Heat transfer for thermoelectric couple ...................................... 38

2.3. Governing equations for TEG module ............................................ 40

2.4. TEG module with hot and cold reservoir.......................................... 43

2.5. Analysis of performance of TEG based self-cooling system................ 45

2.5.1. Overall heat transfer coefficient on hot side ............................... 46

2.5.2. Overall heat transfer coefficient on cold side ............................... 47

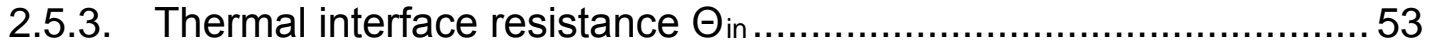

2.5.4. Pressure drop in cold side heat exchanger ................................... 55

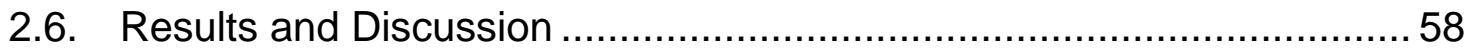

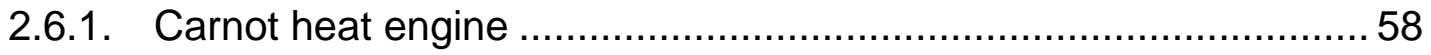

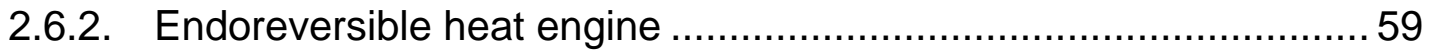

2.6.3. Externally and internally irreversible system ................................ 62

3. EXPERIMENTAL INVESTIGATION ON SELF-COOLING SYSTEM ...........83

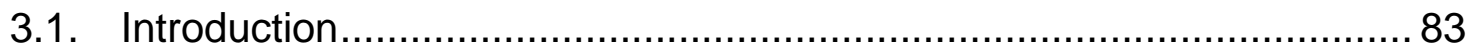

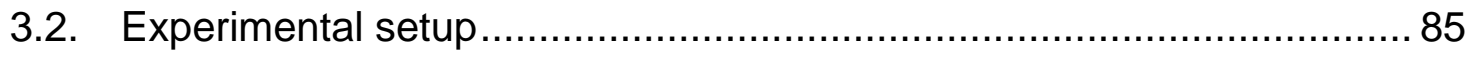

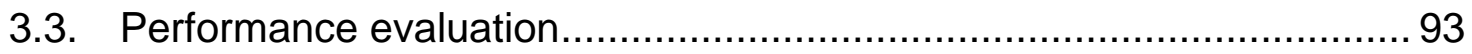

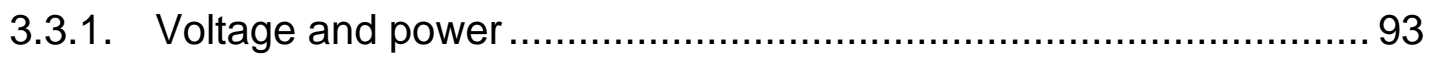




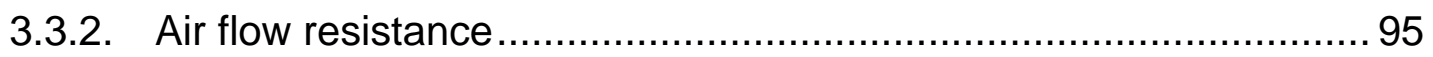

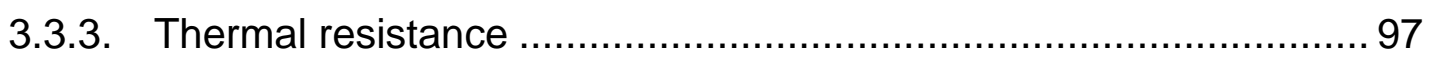

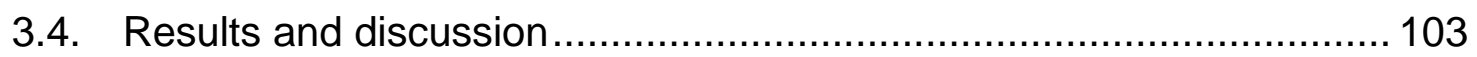

3.4.1. Fan performance in cold-side heat sink ..................................... 103

3.4.2. Voltage and power in case $A$ and $B$.......................................... 105

3.4.3. Dynamic thermal response case $A$ and $B$................................. 109

3.4.4. Application of varying load in case $B$....................................... 113

3.4.5. Comparison between case a-c .............................................. 117

4. NUMERICAL SIMULATION METHODS ON SELF-COOLING SYSTEM .. 123

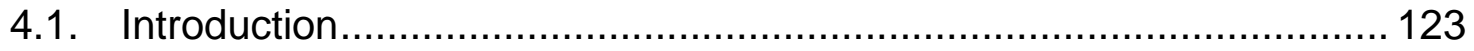

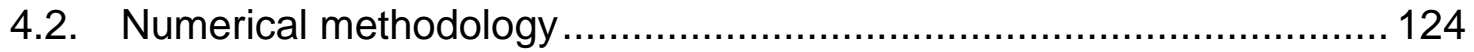

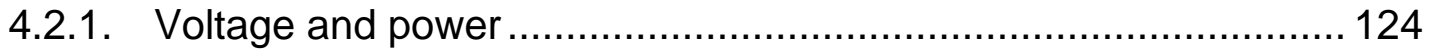

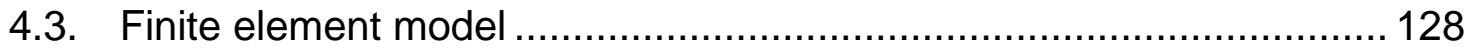

4.3.1. Physical configuration of model ............................................... 128

4.3.2. Equation based modeling in FEM model ................................. 128

4.3.3. Validation of numerical model ................................................. 129

4.3.4. Parametric study using FEM model ......................................... 131

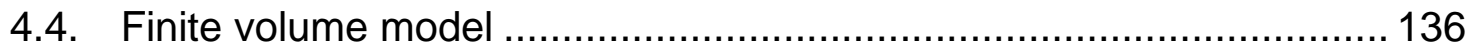

4.4.1. User defined functions based modeling in FVM model ............... 136

4.4.2. Validation of the numerical model.......................................... 145

4.4.3. Parametric study using FVM model ......................................... 153

5. NUMERICAL SIMULATION OF LIQUID COOLING SYSTEM ................. 158

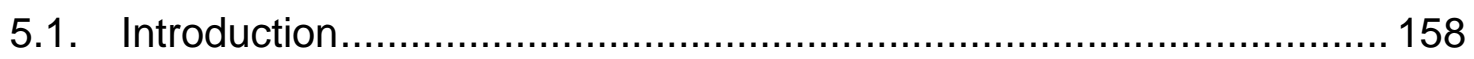

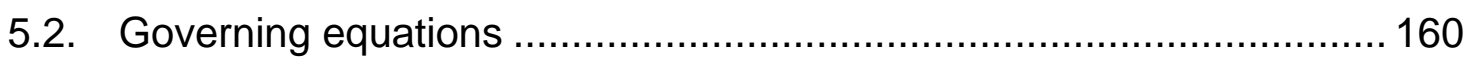

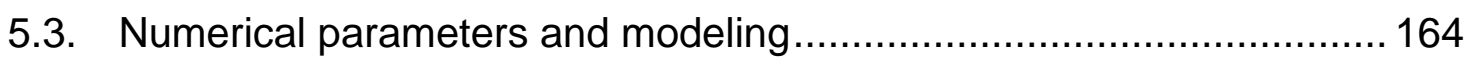

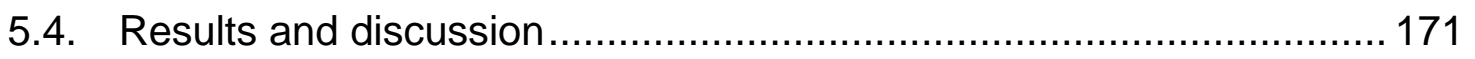

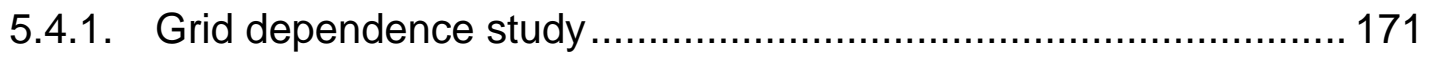

5.4.2. Comparison with experimental data........................................ 174

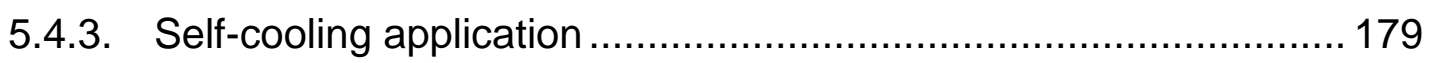

6. DYNAMIC MODELING AND ANALYSIS OF SELF-COOLING SYSTEM.. 185

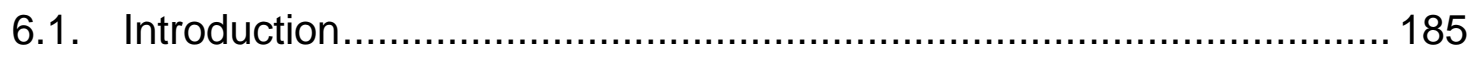

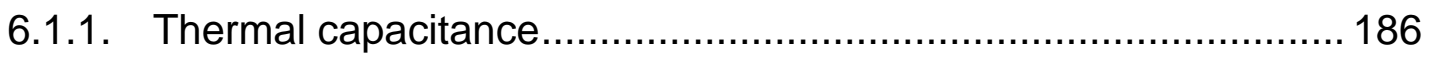




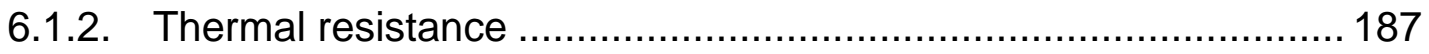

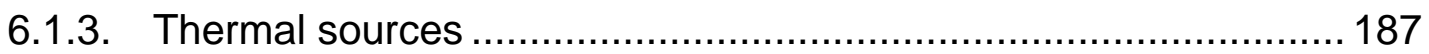

6.2. Electric circuit analogy for lumped body analysis ............................ 188

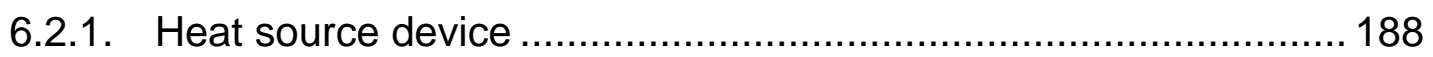

6.2.2. Thermoelectric module (lumped model)................................... 192

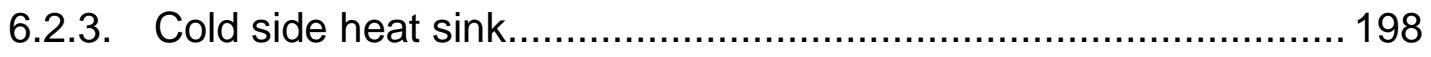

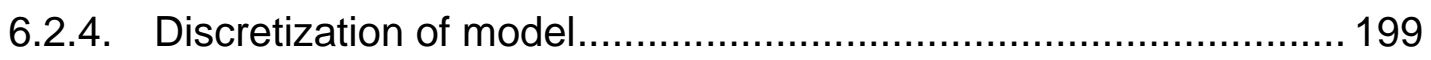

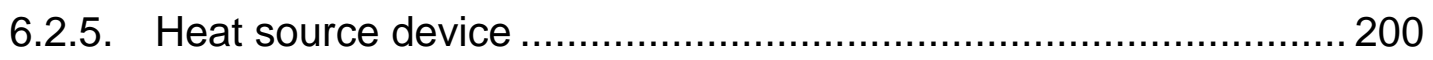

6.2.6. Bottom ceramic plate of TEG module ........................................ 201

6.2.7. TEG cuboid (P-N pellets and conductor) .................................... 202

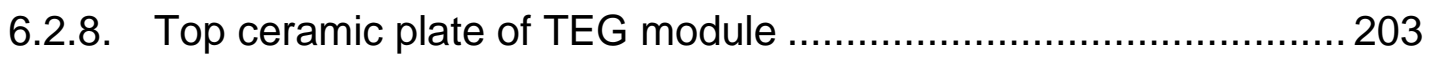

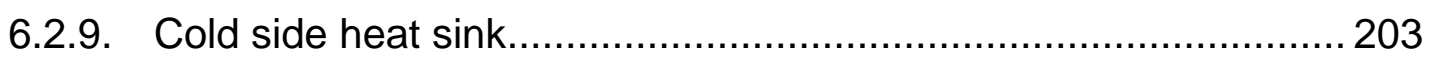

6.3. Modeling electrical system of TEG module ..................................... 204

6.4. Dynamic model implementation in MATLAB/Simulink .......................210

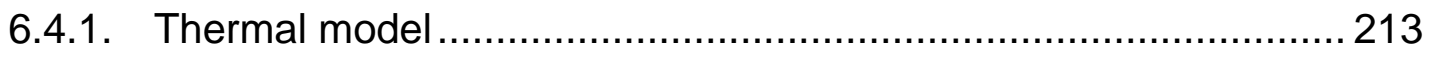

6.4.2. Electrical model.............................................................. 215

6.4.3. Coupling of Cold side heat sink with Electrical model .................. 215

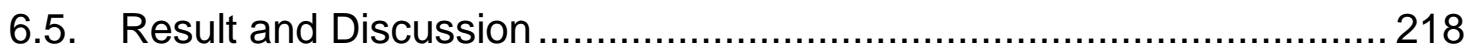

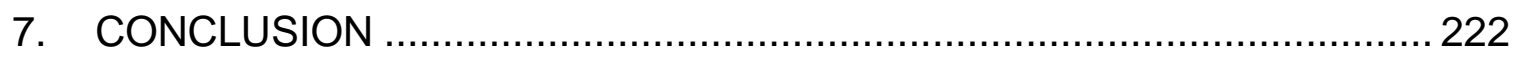

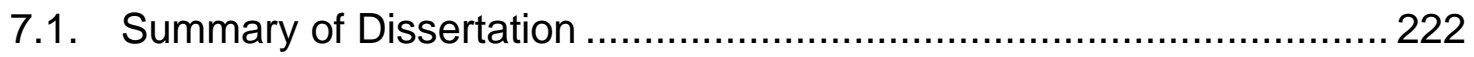

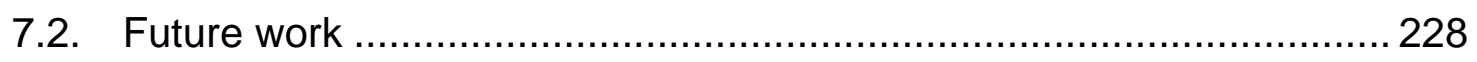

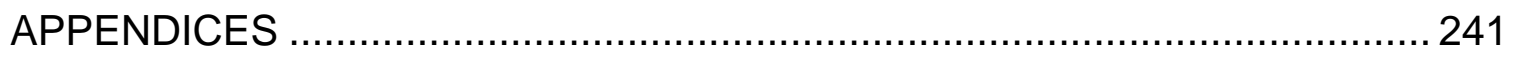

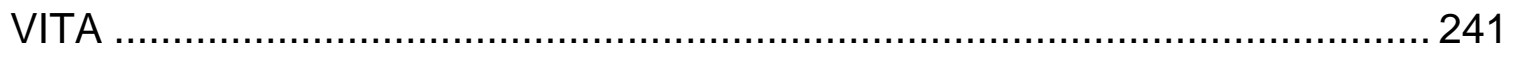




\section{LIST OF TABLES}

TABLE

PAGE

Table 1-1 NSF/DOE Partnership on thermoelectric device application for Vehicles from 2010-2013[16].

Table 3-1 Specification of thermoelectric generator module ( model TEG241-1.0-1.2, China EVERREDTRONICS)

Table 3-2 Specification of DC brushless Fan

( model SUNON EE92251S1-0000-A99).

Table 3-3. Precision of instruments used in the experiments .......................... 92

Table 3-4 Uncertainty in the experimental measurement................................ 93

Table 3-5. Description and expressions for thermal resistances..................... 100

Table 4-1 Physical properties of aluminum, copper and ceramic .................... 139

Table 5-1 Geometrical and modeling input parameter................................. 170

Table 5-2 Comparison between heat applied to the heat sink and heat absorbed by the fluid for different grid sizes for $\Delta$ Tteg $=40^{\circ} \mathrm{C}$ 


\section{LIST OF FIGURES}

FIGURE

PAGE

Figure 1-1 Figure showing a thermoelectric couple in power generation mode.... 4

Figure 1-2 a) Comparison of a) power produced b) cost per kg of thermoelectric material.

Figure 1-3 Comparison of a) material efficiency

b) maximum of hot side temperature. 8

Figure 1-4 Figure showing a thermoelectric generator module 11

Figure 1-5 Graph showing the worldwide funding for

Thermoelectric in 2012[15]

Fig. 1-6.TEG based Self-Cooling Concept ................................................... 17

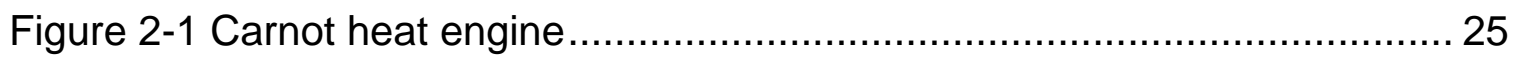

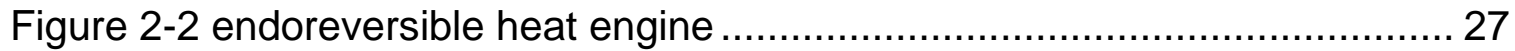

Figure 2-3 Externally and internally irreversible heat engine .......................... 30

Figure 2-4 Parallel plate-fin heat exchanger ................................................. 57

Figure 2-5 variation of $\eta_{c}$ with the ratio of heat sink to heat source temperature 59

Figure 2-6 Comparison of Carnot heat engine and endoreversible heat engine 61

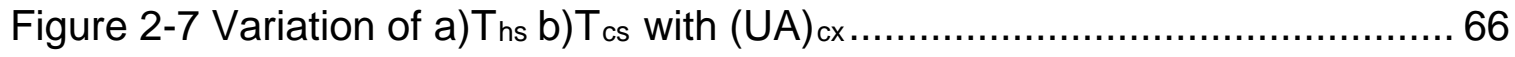

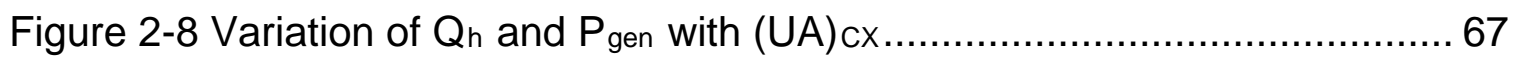

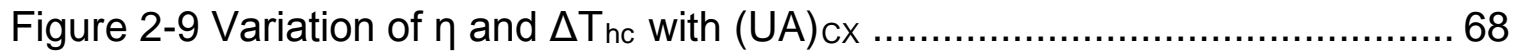

Figure 2-10 Comparison of $V_{o c}$ and I for single TEG module and array of

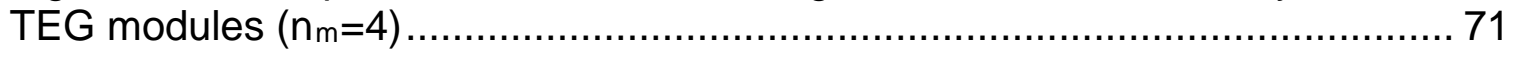

Figure 2-11 Comparison of $\mathrm{Q}_{\mathrm{h}}$ and $\mathrm{Pgen}$ for single TEG module and

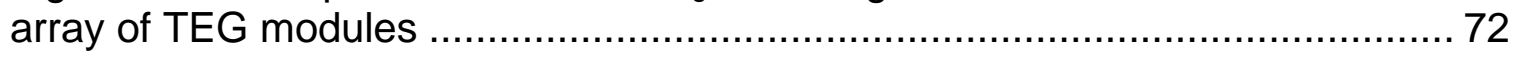

Figure 2-12 Comparison of single TEG module and array of modules for a) $T_{h s}$ b) $T_{c s}$ 
Figure 2-13 Variation of Reynolds number $(\operatorname{Re})$ and pressure drop $(\Delta \mathrm{P})$ with $(\mathrm{UA}) \mathrm{cx}$ 77

Figure 2-14 Variation of $T_{h s}$ and $T_{c s}$ with (UA)cx 77

Figure 2-15 Variation of Voltage and Current produced by array of modules with $(\mathrm{UA}) \mathrm{cx}$ 78

Figure 2-16 Variation of power generated by TEG arrays $\left(P_{\text {gen }}\right)$, power consumed by fluid mover $\left(P_{\text {con }}\right)$ and net power in the system $\left(P_{\text {net }}\right)$..... 80

Figure 2-17 Self-cooling window. 81

Figure 3-1 Geometrical configurations of cases A-C 86

Figure 3-2 a) picture of experimental setup and b) schematics of the investigation system 88

Figure 3-3 Thermal resistance diagram. 99

Figure 3-4 Fan-heat sink operation curve for case A and B 104

Figure 3-5 Temperature variations as a function of time for case $A$ and Qin of a) $70 \mathrm{~W} \mathrm{b)} 80 \mathrm{~W} \mathrm{c)} 90 \mathrm{~W}$ and d) $100 \mathrm{~W}$ 106

Figure 3-6 a) Total array Voltage and b) Power per unit area of TEG (PIATEG) and I as a function of Qin for case A .

Figure 3-7 a) Total array Voltage and b) Power per unit area of TEG (P/ATEG) and Current as a function of Qin for case B

Figure 3-8 Temperature difference $\left(\Delta T_{\text {de }}\right)$ between the device temperature $\left(T_{d}\right)$ and environment temperature $\left(T_{e}\right)$ for case $A$ at varying heat input $\left(Q_{\text {in }}\right)$ of a) $\left.50-80 \mathrm{~W} \mathrm{~b}\right) 90-120 \mathrm{~W}$.

Figure 3-9 Temperature difference $\left(\Delta T_{\text {de }}\right)$ between the device temperature $\left(T_{d}\right)$ and environment temperature $\left(T_{e}\right)$ for case $B$ at varying heat input $Q_{\text {in }}$ of a) $60-90 \mathrm{~W} \mathrm{b)} \mathrm{100-130} \mathrm{W}$ 116

Figure 3-10 Variation of device temperature (Td) for varying heat input load (Qin) a) S1 b) S2 c) S3...

Figure 3-11 Variation of a) Total thermal resistance, $\Theta_{\mathrm{th}, \mathrm{T}}$ b) $\Delta \mathrm{T}_{\mathrm{de}} \ldots \ldots \ldots \ldots \ldots \ldots . . . . . .121$

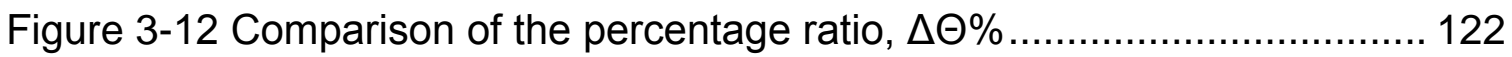


Figure 4-1 Numerical Methodology

Figure 4-2 Comparison between numerical simulation model and experimental data

Figure 4-3 . Cooling fan performance and power consumed by the fan as a function of volume rate of air in the fan for different fin densities.

Figure 4-4 Maximum temperature of the heated plate, $\mathrm{Tg}$ and power produced by the TEG, Pgen as a function of heating power supplied to the heater.

Figure 4-5 Cooling fan performance and power consumed by the fan as a function of volume rate of air in the fan for different fin densities.

Figure 4-6 Maximum temperature of the heated plate and power produced by the TEG, $P_{g e n}$, as a function of heating power supplied to the heater. ........ 135

Figure 4-7 Computational domain A and B in ANSYS ................................. 141

Figure 4-8 a) Schematic diagram of numerical model b) computational grid of TEG module

Figure 4-9 Fan performance data and system operation curve for case $A$ and $B$

Figure 4-10 Average convection heat transfer coefficient ( $h_{\text {av }}$ ) as a function of voltage

Figure 4-11 a) Total array voltage ( $\mathrm{V}$ ) and Power produced by TEG arrays per area of TEG arrays (PTEG/ATEG,a) for a) Case A and b) Case B ....... 149

Figure 4-12 Transient simulation of a) voltage supplied to fan b) temperature difference between device and ambient temperature

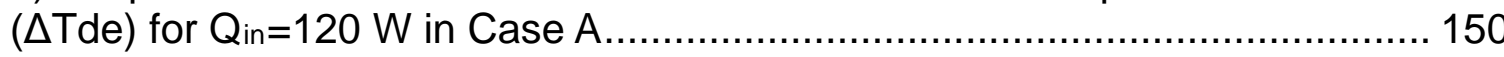

Figure 4-13 Temperature distribution $Q=120 \mathrm{~W}$ a) case $\mathrm{A}$ b) case $\mathrm{B}$ after 60 minutes

Figure 4-14 Temperature difference between hot and cold side of TEG modules $(\Delta \mathrm{T}$ de $)$.

Figure 4-15 Effect of load electrical resistance (Rel,L) a) case A b) case B..... 154

Figure 4-16 Variation of $\mathrm{P}_{\mathrm{TEG}} / \mathrm{A}_{\mathrm{TEG}, \mathrm{a}}$ and total thermal resistance $\left(\Theta_{\text {th }}\right)$ as a function of fin height $\left(\mathrm{H}_{\text {fin }}\right)$. 155 
Figure 4-17 Variation of $\mathrm{P}_{\mathrm{TEG}} / \mathrm{A}_{\mathrm{TEG}, \mathrm{a}}$ and total thermal resistance $\left(\Theta_{\mathrm{th}}\right)$ as a function of fin density $\left(\mathrm{H}_{\text {fin }}\right)$......

Figure 5-1. A schematic diagram of a microchannel heat sink based self-cooling system

Figure 5-2 Equivalent a) Electrical resistance model of TEG and Pump

b) hydraulic resistance model c) thermal resistance model 165

Figure 5-3. Computational model parts and configurations 169

Figure 5-4. a) Computational grid b) Channel designation for half part of the microchannel heat sink

Figure 5-5. The variation of temperature as a function of $D C L$ for channel \#6 . 174

Figure 5-6. Average pressure loss $\left(\Delta P_{a v}\right)$ for the microchannel as a function of coolant flow rate.

Figure 5-7. Comparison between numerical modelling and experimental data for the coolant temperature difference in channel \#6 between the inlet and outlet port as a function of cooling flow rate.

Figure 5-8. The variation of net power with coolant flow rate for different $\Delta \mathrm{T}_{\text {teg }} 178$

Figure. 5-9. Maximum generated power $\left(\mathrm{P}_{\text {gen }}\right)$, pumping power $\left(\mathrm{P}_{\text {pump }}\right)$ and net power $\left(P_{n e t}\right)$ for q" $=20 \mathrm{~W} / \mathrm{cm}^{2}$ 180

Figure 5-10. Variation of net power and junction temperature with heat flux and cooling flow rates

Figure. 5-11. Variation of thermal and hydraulic resistance with cooling flow rate

Figure. 5-12. Comparison of modeled Pressure flow characteristic and power requirement with commercial micropump 184

Figure. 6-1 Heat transfer in heat source device 190

Figure. 6-2 Electrical circuit analogy of heated device. 191

Figure. 6-3 TEG module compact model 193

Figure. 6-4 Electrical circuit analogy of bottom ceramic plate 194

Figure. 6-5 Electrical circuit analogy of TEG module 
(P-N pellets and conductor) 196

Figure. 6-6 Electrical circuit analogy of top ceramic plate............................ 198

Figure. 6-7 Electrical circuit analogy of cold side heat sink ......................... 200

Figure. 6-8 Discretized form of heat source device .................................... 201

Figure. 6-9 Discretized form of bottom ceramic plate .................................. 202

Figure. 6-10 Discretized form of TEG module (P-N pellets and conductor) ...... 203

Figure. 6-11 Discretized form of top ceramic plate ...................................... 204

Figure. 6-12 Discretized form of cold side heat sink .................................... 204

Figure. 6-13Coupling of electrical and thermal model ................................. 205

Figure. 6-14 P and N type legs with air gap................................................ 208

Figure. 6-15 Main workspace for Simscape modeling ................................. 214

Figure. 6-16 a) Device subsystem divided in to 5 subsystems (Dev1-Dev 5).. 216

Figure. 6-17 TEG thermal subsystem ................................................... 217

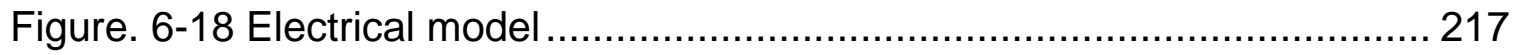

Figure. 6-19 Comparison of Dynamic model result for $\Delta T_{\text {de }}\left(T_{d}-T_{\text {env }}\right)$

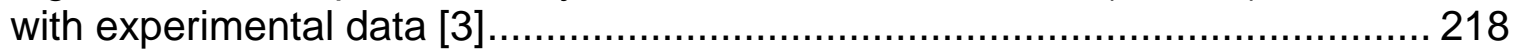

Figure. 6-20 Comparison of Dynamic model result for $\Delta T_{\text {de }}\left(T_{d}-T_{\text {env }}\right)$ with ....... 219

Figure. 6-21 Comparison of Dynamic model result for $\Delta T_{\text {de }}\left(T_{d}-T_{\text {env }}\right)$

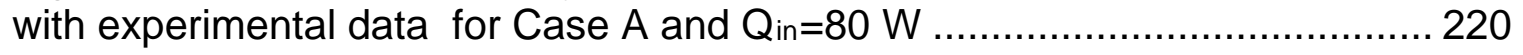

Figure 6-22 Transient variation of voltage for Case A in Qin=80 W................ 220 


\section{INTRODUCTION}

\subsection{Background}

The rising cost of energy and increasing environmental awareness have given prominence to research focusing on the use of thermoelectric generator (TEG) modules for various waste heat to electricity applications [1]. These include aerospace, automobiles, solar energy systems, wireless sensors and wood stoves [2], [3], [4], [5], [6], [7].

Thermoelectric generation is a promising technology that cleanly converts waste heat into electricity. A TEG (thermoelectric generator) module consists of a number of semi-conductor pairs connected in series by copper solders and contained between thermally conductive and electrically insulated ceramic plates. They enable the change of a temperature difference across the hot side and cold side to be directly converted to electricity using the Seebeck effect.

TEG modules could also be applied in self-driven cooling loops by using waste heat from a hot device. The heat, which is normally removed from hot surfaces, can be converted to an electrical power using TEG modules. The power could then be used to self-sustain a cooling system for the hot bodies. This helps save power by reducing the need for external power and is also suitable for remote area application where autonomous and reliable power supply is essential. 
A self-cooling system could be thought of as a forced convection cooling system without an external power supply. It could potentially be a useful cooling mechanism for applications where the system (device) may not be adequately cooled by natural convection. Thus, there is a motivation to replace external power sources or utilize self-cooling where external power sources are not available or feasible.

This chapter discusses the fundamental concepts of thermoelectric generation, followed by a review of the uses of thermoelectric generation application in a waste heat scavenging focusing on uses that pertain to self-cooling.

\subsection{Review on thermoelectrics}

Thomas Seebeck, a German physicist, discovered in 1821 that a magnetic field is developed between two dissimilar electrical conducting materials when they are connected at their legs and a temperature difference is maintained between the ends. He did not, however, explain the cause of the phenomenon. It is presently known as the Seebeck effect.

A thermoelectric material contains free electrons or holes that carry charge and heat. When one end of the material is maintained at a higher temperature, the charge carriers move faster and migrate to the cold side. This in turn creates high density of charge carriers at the cold side, which forces them to diffuse back to 
the hot end. The build-up of charge carriers at the cold side creates an electric potential difference in what is commonly known as the Seebeck effect.

A proportionally constant between temperature difference $(\Delta T)$ and electric potential $(V)$ is known as the Seebeck coefficient $(\alpha)$. If the material builds up positive charges (hole charge carriers) at the cold side and has positive potential, it is called p-type material. On the other hand, n-type material carries negative free charges (electron charge carriers), exhibits build-up negative charges at the cold side and has negative potential. In pure semiconductor materials, at higher temperature, there will be n-type carries in the conduction band and p-type accrues in the valence band. The presence of both p-type and n-type carries reduces the resultant Seebeck coefficient, as both effects cancel each other. Thus, materials are usually heavily doped to produce more of one of the carriers (p-type or n-type).

The Seebeck effect can be expressed by the equation:

$$
V_{o c}=\left(\alpha_{p}-\alpha_{n}\right)\left(T_{h s}-T_{c s}\right)
$$

where $V_{o c}$ is the open circuit voltage produced due to the Seebeck effect, $\alpha_{p}$ and $\alpha_{n}$ are the Seebeck coefficients of p-type and n-type legs, respectively, and $T_{h s}$ and $T_{c s}$ are the hot and cold side temperatures of the end of the semiconductor 
legs. The unit of the Seebeck coefficient is usually given as $\mathrm{mV} / \mathrm{K}$. It should be noted that the Seebeck coefficient is usually temperature dependent and the derivation of a more comprehensive thermoelectric is described in the next chapter.

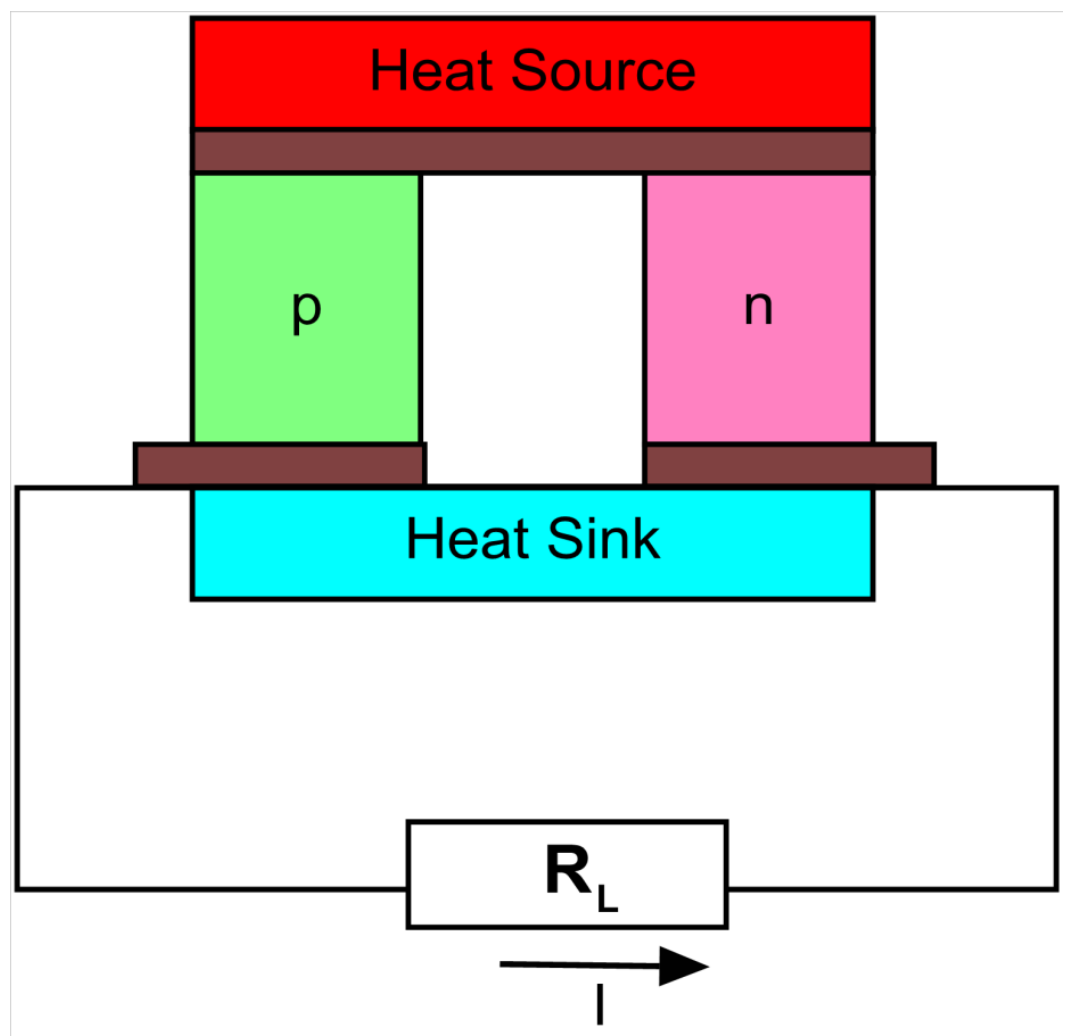

Figure 1-1 Figure showing a thermoelectric couple in power generation mode

Thermoelectric couples when connected to a load with electrical resistance of $R \mathrm{~L}$, as shown in Figure 1-1. It produces power, which is given as:

$$
P_{L}=I^{2} R_{L}
$$


There is also an opposite effect that is known as a Peltier effect, which was discovered by a French physicist Jean-Charles-Athanase in 1834 . He noted that a current passing through a pair of dissimilar conducting materials creates heat flow from one side to another and also observed that the heat flow was more than what is expected from Ohmic heating. His observation was later named as the Peltier effect.

A figure of merit is a common term that is used to describe the performance of a thermoelectric material. The figure of merit, $\mathrm{z}$ (unit $\mathrm{K}^{-1}$ ), of a thermoelectric material is defined as:

$$
z=\frac{\alpha^{2} \sigma}{k}
$$

It is also common to find it in a literature defined as dimensionless by multiplying with temperature:

$$
z T=\frac{\alpha^{2} \sigma}{k} T
$$

It is a function of three parameters: electrical conductivity $\sigma$, Seebeck coefficient $\alpha$ and thermal conductivity $k$. The figure of merit indicates that a material 
produces more electrical potential between the ends with an increase in the Seebeck coefficient. However, $\mathrm{z}$ is also indirectly proportional to the conductivity of the material. This is due to the need to maintain a larger temperature difference between the ends of the thermoelectric material. However, many electrical conductive materials also have high thermal conductivity.

Figure 1-2 and Figure 1-3 (adapted from [8]) show the comparison between different materials according to cost and performance. The data was compiled for the DOE workshop in 2009. As could be observed from Figure 1-2a, $\mathrm{Bi}_{2} \mathrm{Te}_{3}$ had the highest power output per $\mathrm{kg}$ of mass of a material $\left(221 \mathrm{~W} / \mathrm{kg}\right.$ when $10 \mathrm{~W} / \mathrm{cm}^{2}$ of heat flux is used). For the cost of the materials (Figure 1-2b), Skutterdites had the least cost (12 dollars per $\mathrm{kg}$ ), which comes to 0.2 dollars per watt. $\mathrm{Bi}_{2} \mathrm{Te}_{3}$ had around the cost of 0.8 dollars per watt of power produced. When it comes to material efficiency (Figure 1-3a), Segmented materials had the highest efficiency compared to the other materials in the figure. $\mathrm{Bi}_{2} \mathrm{Te}_{3}$ has an average efficiency of around 7.1 with maximum temperature of around $525 \mathrm{~K}\left(252^{\circ} \mathrm{C}\right)$.

In this thesis, the thermoelectric effect is applied mainly in relation to the cooling of electronic devices. With the ambient temperature of around $25{ }^{\circ} \mathrm{C}$, the temperature range under consideration is in the range of 25 to $200{ }^{\circ} \mathrm{C}$. A most common and efficient material for the temperature range under consideration is Bismuth Telluride $\left(\mathrm{Bi}_{2} \mathrm{Te}_{3}\right)[9]$. 


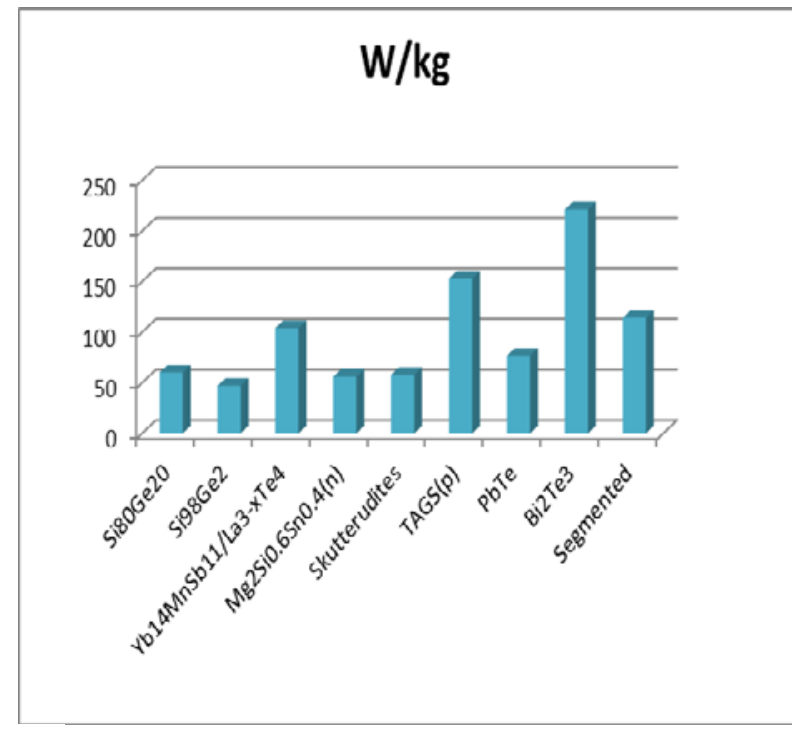

a)

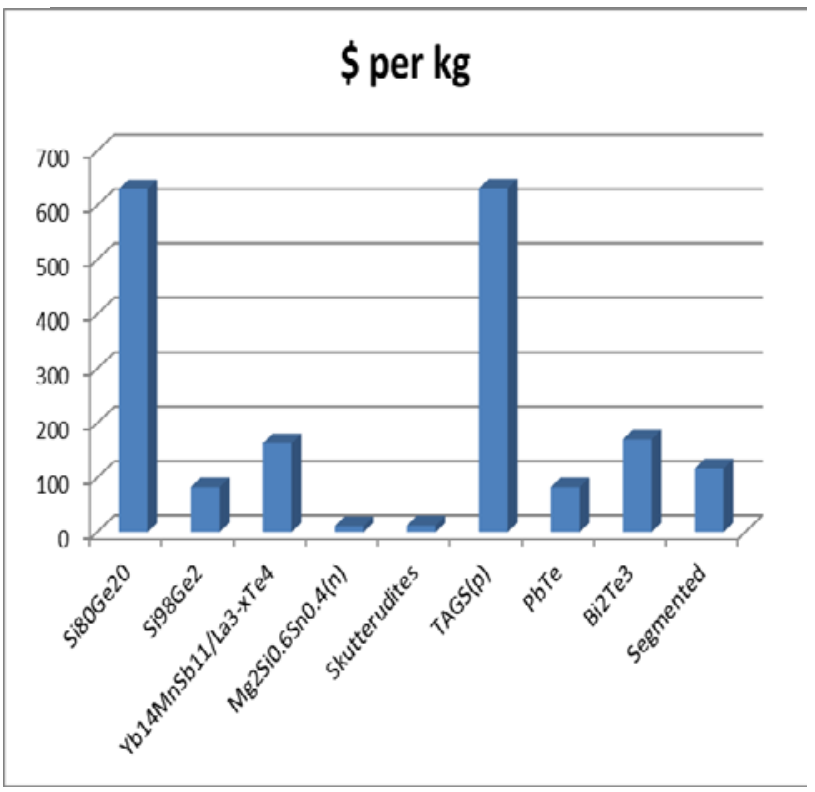

b)

Figure 1-2 a) Comparison of a) power produced b) cost per ka of thermoelectric material. 


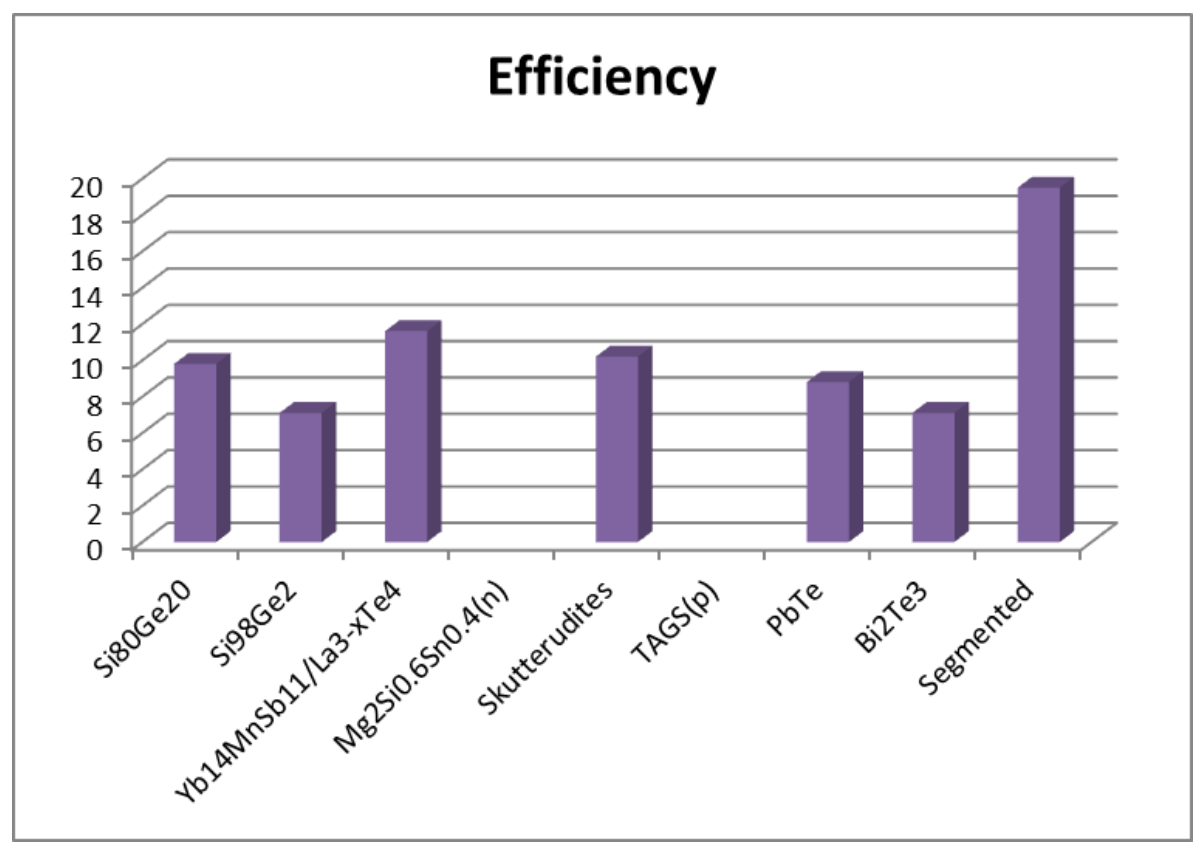

a)

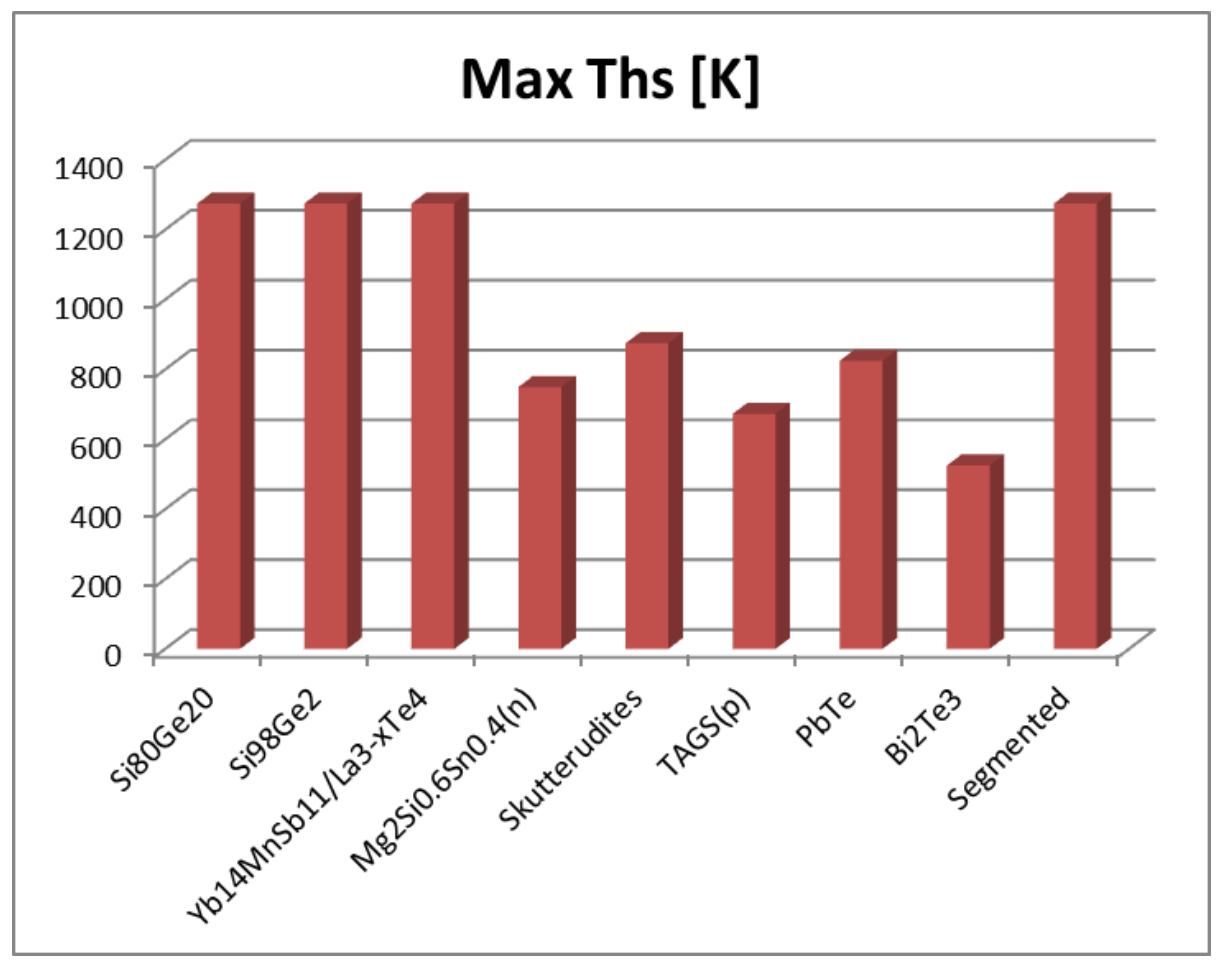

b)

Figure 1-3 Comparison of a) material efficiency b) maximum of hot side temperature. 
For a thermoelectric couple, the expression for figure of merit for a single leg (Eq. 1.2.4) could be rewritten as:

$$
z T=\frac{\left(\alpha_{p}-\alpha_{n}\right)^{2}}{\left(\sigma_{p}^{-1} k_{p}\right)^{1 / 2}+\left(\sigma_{n}^{-1} k_{n}\right)^{1 / 2}} T
$$

where the properties at the respective legs are denoted by the subscripts of $p$ and $\mathrm{n}$.

The efficiency of thermoelectric couple ( $\left.\eta_{T E}\right)$ is described by the power produced at the load per unit of heat flow rate into the thermoelectric couple. It could be written as:

$$
\eta_{T E}=\frac{P_{L}}{Q_{i n}}
$$

where $\mathrm{P}_{\mathrm{L}}$ is the power input to a load and $\mathrm{Qi}_{\mathrm{n}}$ is the heat flow into the thermoelectric couple.

Eq. 1.2.6 could also be rewritten in terms of figure of merit $(\mathrm{zT})[10]$ as:

$$
\eta_{T E}=\frac{T_{h s}-T_{c s}}{T_{h s}}\left(\frac{\left(1+z T_{m}\right)^{1 / 2}-1}{\left(1+z T_{m}\right)^{1 / 2}+T_{c s} / T_{h s}}\right)
$$


where $T_{m}$ is the average temperature of the semiconductor legs. The first term in the right-hand side of the equation is actually the Carnot efficiency of an equivalent heat engine.

Solid state thermoelectric generation (TEG) modules don't have any moving parts or working fluid inside the parts and have high reliability, compact design and noiseless operation. As shown in Figure 1-4, a thermoelectric module consists of thermoelectric semiconductor pairs connected in series electrically and in parallel thermally. The semiconductor pairs are connected at the ends using a conductor. A thermally conductive but electrically insulating material is placed on the top and bottom of the thermoelectric legs.

The solid state TEG module is highly scalable and modular. It has no vibrations and can outperform competition for small-scale applications. Thus, there is a potential for utilization of waste heat by system integration or interfacing with the industrial process. These may include the single-phase Rankine cycle, mixedfluid cycle, or combined cycles.

\subsection{Review on Waste Heat Scavenging}

Waste heat recovery had its early uses in space in what are known as radioisotope thermoelectric generators (RTGs), where the waste heat from nuclear decay of radioactive isotopes was used [11]. 


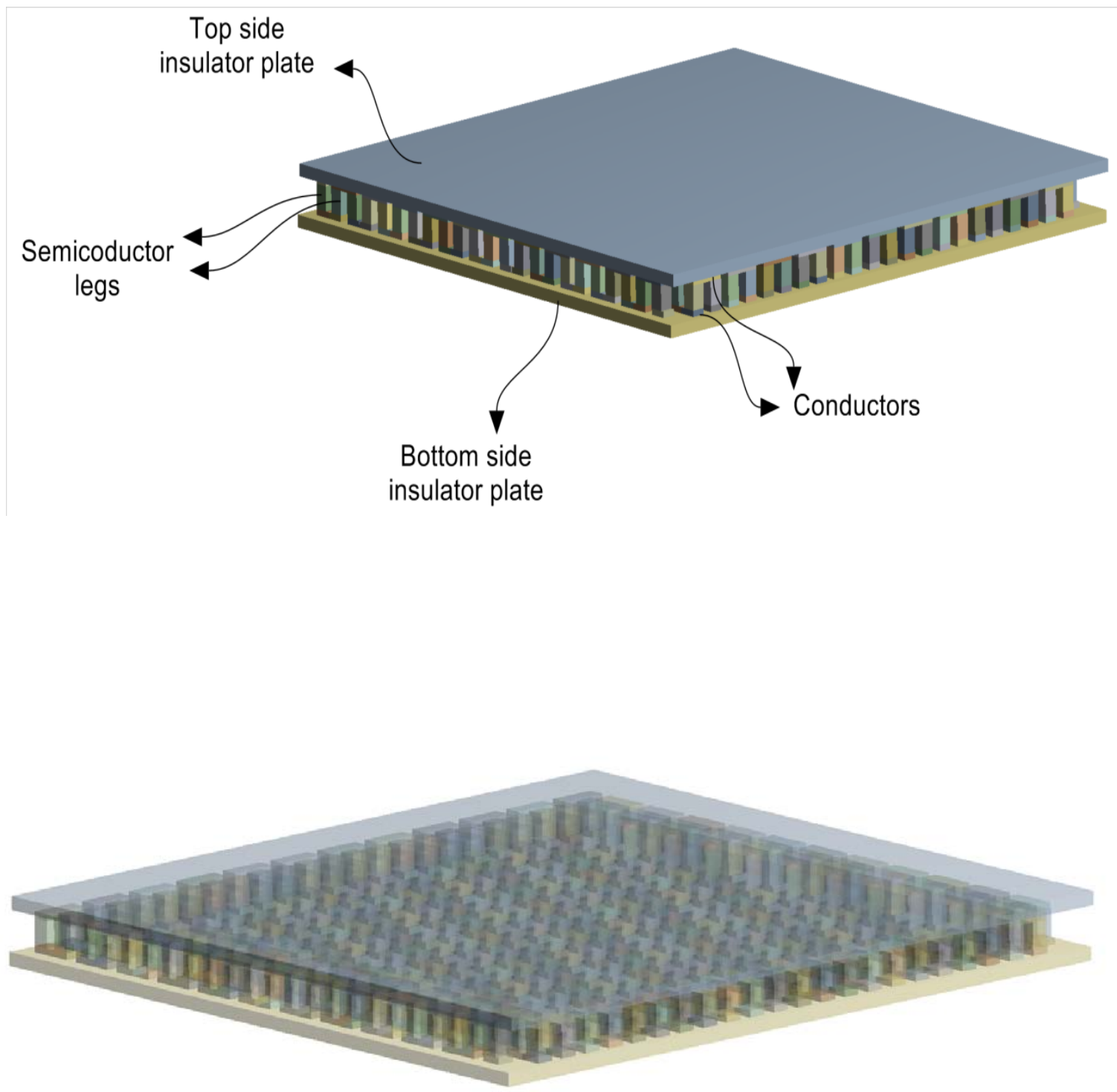

Figure 1-4 Figure showing a thermoelectric generator module

The applications in space had been in the high temperature range, which is usually around $1000{ }^{\circ} \mathrm{C}$. Thus, high temperature thermoelectric materials like 
Silicon Germanium were typically used. There are also numerous heat sources for terrestrial applications, which could be low-level heat sources $\left(<250^{\circ} \mathrm{C}\right)$, midlevel heat sources $\left(\sim 250-650{ }^{\circ} \mathrm{C}\right)$ and high-level heat sources $\left(>650{ }^{\circ} \mathrm{C}\right)$ [12]. Applications that are suitable for waste heat recovery include vehicles, industrial processes, and renewable energy sources like Photovoltaic cells, human body and electronic devices. Thermoelectric application for reducing the environmental impact of other power sources has also been demonstrated [13][14].

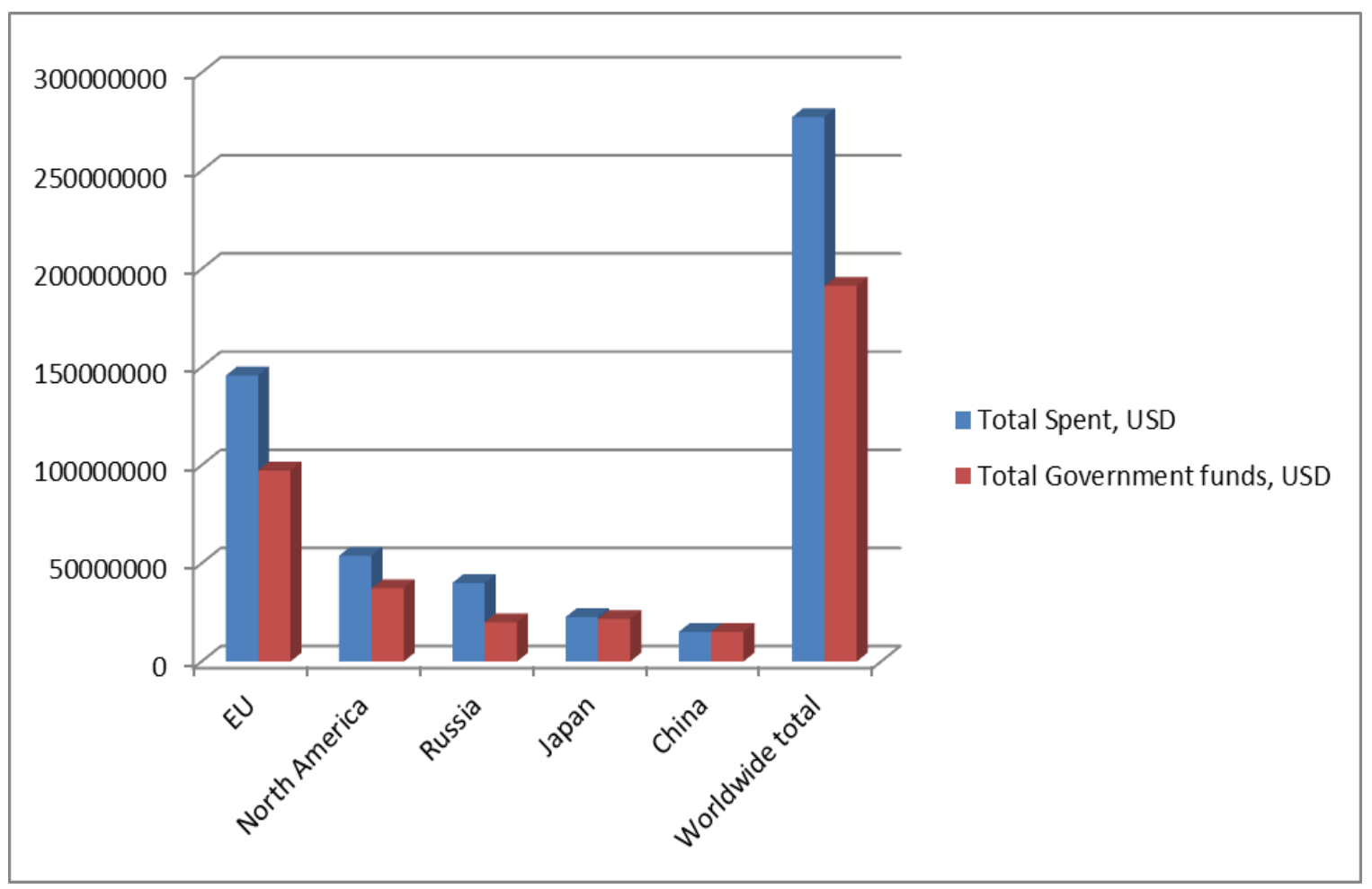

Figure 1-5 Graph showing the worldwide funding for Thermoelectric in 2012[15] 
A report by Gentherm[15] showed that the worldwide expenditure on research on thermoelectric was around 277 million in 2012 and of that, almost half of the funding came from the European Union followed by North America (20\%).

US Department of Energy (DOE)[16] detailed projects done with the NSF/DOE partnership to study the application of thermoelectric devices for vehicle applications. The details of projects funded by DOE for the time period of 201013 is shown in Table 1-1.

Hendricks et al[17] demonstrated the TEG system for heat recovery from diesel generators' exhaust. Liu et al. [18] experimentally and numerically analyzed an energy-harvesting system for automotive exhaust pipes using TEG modules. Hsu et al [19] experimentally and numerically studied waste heat harvesting by TEG modules from an automobile exhaust pipe. For an engine boosts of 3500 RPM, it was claimed that a maximum power output of $12.41 \mathrm{~W}$ was obtained for an average temperature difference of $30 \mathrm{~K}$.

Kuroki et al[20] described a TEG system using waste heat from the steel-making processes. Their system consisted of 16 TEG modules with each module capable of generating $18 \mathrm{~W}$ with a hot side temperature of $523 \mathrm{~K}$. Thermoelectric generators have also been integrated with photovoltaic cells in a solar-driven hybrid generation system to convert the heat collected by thin-film solar cells to electricity [21]. 
Table 1-1 NSF/DOE Partnership on thermoelectric device application for Vehicles from 2010-2013[16]

\begin{tabular}{|c|c|c|}
\hline Project Title & $\begin{array}{l}\text { Academic PIs/ } \\
\text { Universities }\end{array}$ & $\begin{array}{l}\text { Industrial } \\
\text { collaborator }\end{array}$ \\
\hline $\begin{array}{l}\text { Automotive Thermoelectric Modules } \\
\text { with Scalable Thermo- and Electro- } \\
\text { Mechanical Interfaces }\end{array}$ & $\begin{array}{l}\text { K.E. Goodson (Stanford University), } \\
\text { G. Nolas (University of South } \\
\text { Florida) }\end{array}$ & $\begin{array}{l}\text { B. Kozinsky (Robert } \\
\text { Bosch LLC) }\end{array}$ \\
\hline $\begin{array}{l}\text { An integrated approach towards efficient, } \\
\text { scalable, and low cost thermoelectric } \\
\text { waste heat recovery devices for vehicles }\end{array}$ & $\begin{array}{l}\text { Scott T. Huxtable, Srinath V. Ekkad, } \\
\text { and Shashank Priya, Virginia Tech }\end{array}$ & $\begin{array}{l}\text { Andrew Miner, Romny } \\
\text { Scientific, San Bruno, } \\
\text { CA }\end{array}$ \\
\hline $\begin{array}{l}\text { High-Performance Thermoelectric } \\
\text { Devices Based on Abundant Silicide } \\
\text { Materials for Vehicle Waste Heat } \\
\text { Recovery }\end{array}$ & $\begin{array}{l}\text { Li Shi, John B. Goodenough, Matt J. } \\
\text { Hall, Jianshi Zhou (U. of Texas at } \\
\text { Austin); Song Jin (U. of Wisconsin- } \\
\text { Madison) }\end{array}$ & \\
\hline $\begin{array}{l}\text { High performance TE system based on } \\
\text { Zintl phase materials with embedded } \\
\text { nanoparticles }\end{array}$ & $\begin{array}{l}\text { A. Shakouri (Purdue); Z. Bian (UC } \\
\text { Santa Cruz); S. M Kauzlarich (UC } \\
\text { Davis) }\end{array}$ & NASA JPL \\
\hline $\begin{array}{l}\text { Integration of Advanced Materials and } \\
\text { Interfaces for Durable Thermoelectric } \\
\text { Automobile Exhaust Waste Heat } \\
\text { Harvesting Devices }\end{array}$ & $\begin{array}{l}\text { Y. Sungtaek Ju and Bruce Dunn } \\
\text { (UCLA) }\end{array}$ & JPL \\
\hline $\begin{array}{l}\text { GM Partnership on Thermoelectrics for } \\
\text { Automotive Waste Heat Recovery }\end{array}$ & $\begin{array}{l}\text { Xianfan Xu, Timothy S. Fisher, } \\
\text { Steven D. Heister, Yue Wu, } \\
\text { Timothy D. Sands, Purdue } \\
\text { University }\end{array}$ & $\begin{array}{l}\text { Gregory Meisner, } \\
\text { James Salvador, } \\
\text { General Motors R\&D }\end{array}$ \\
\hline
\end{tabular}

He et al. [22] showed that TEG modules could be directly incorporated with evacuated-tube heat-pipe solar collectors to get a higher heat flux into the modules. Sodano et al[23] studied a Seebeck heat pump based on TEG module to convert the thermal gradient by solar radiation and waste heat. The hot side of the module was placed on a greenhouse and the cold side on a highway bridge. 
The system was used to recharge a battery. Chen[24] investigated the theoretical efficiency of solar thermoelectric generators (STEG), which included a model with thermal and optical concentration.

Muhtaroglu et al[25] proposed the application of TEG and Photovoltaic (PV )as a viable power source for a mobile computing system. The system benefited from extended battery life. They mentioned that using energy sources in the vicinity of a computing system was a viable method to extend on-board power. However, their research mentioned the challenges associated with stepping up the lowvoltage output to enable integration with the system battery. It was, however, indicated that the future power management breakthroughs would be related to scavenging energy to enhance power of on-board electronics systems. Kim et al[26] studied the recovery of waste heat from power amplifier transistors in telecommunication networks.

Tritt et al[27] described a direct solar thermal conversion method in which concentrated solar rays could be separated into different spectrums. According to the research, the UV spectrum ( $200-800 \mathrm{~nm})$ could be used for PV applications while the IR spectrum ( $800-3000 \mathrm{~nm})$ could be utilized in TEG modules. TEG modules have also been utilized for remote area applications that include weather stations, oil drilling platforms (cathodic corrosion protection) and oil and gas pipelines[28]. 
Thermoelectric generators could also be used in "self-powered" applications in which the system drives its power from TEG modules. Carmo et al[29] demonstrated the application of a TEG microconverter to scavenge temperature difference from body heat and ambient to power electroencephalogram (EEG) modules. The power consumption for EEG modules was up to a few milliwatts. Wang et al [30] demonstrated miniaturized wearable TEG modules for application using the human body. The wearable TEGs were able to produce an open-circuit voltage of $12.5 \mathrm{~V} /\left(\mathrm{K} \mathrm{cm}^{2}\right)$ and power of $0.026 \mu \mathrm{W} /\left(\mathrm{K}^{2} \mathrm{~cm}^{2}\right)$. Torfs et al[31] manufactured an autonomous wireless EEG powered by TEG modules working between the human body and ambient. The whole system consumed less than $0.8 \mathrm{~mW}$ and TEG modules produced over $2 \mathrm{~mW}$ at $23^{\circ} \mathrm{C}$. There has also been other research on wearable wireless self-powered devices [32][33].

Shi et al. [6] experimentally studied the performance of the TEG-based selfpowered wireless temperature sensor, which enables the sensor to function without batteries or other power sources. The Seiko theramic wristwatch utilizes the heat from human wrist to run a wrist watch using ten TEG modules[34]. However, the temperature difference between the human skin and the atmosphere was so low that TEG modules needed to cover a large area to produce an output voltage of more than $1 \mathrm{~V}$. The typical voltage produced by a TEG module working between the human skin and the atmosphere is in the range of micro to milliwatts. 


\subsection{Review on Thermoelectric Generator-Based Self-cooling Applications}

One ingenious application of thermoelectric technology is to integrate thermoelectric generation and electronic device cooling into one system in what it known as thermoelectric-based self-cooling application. Fig. 1-6 illustrates the self-cooling concept. The basic idea is to convert the heat, which is normally removed from hot surfaces to an electrical power. This could then be used to selfsustain a cooling system for the hot bodies via a thermoelectric generation technology. This helps save power by reducing the need for external power and is also suitable for remote area applications where autonomous and reliable power supply is essential.

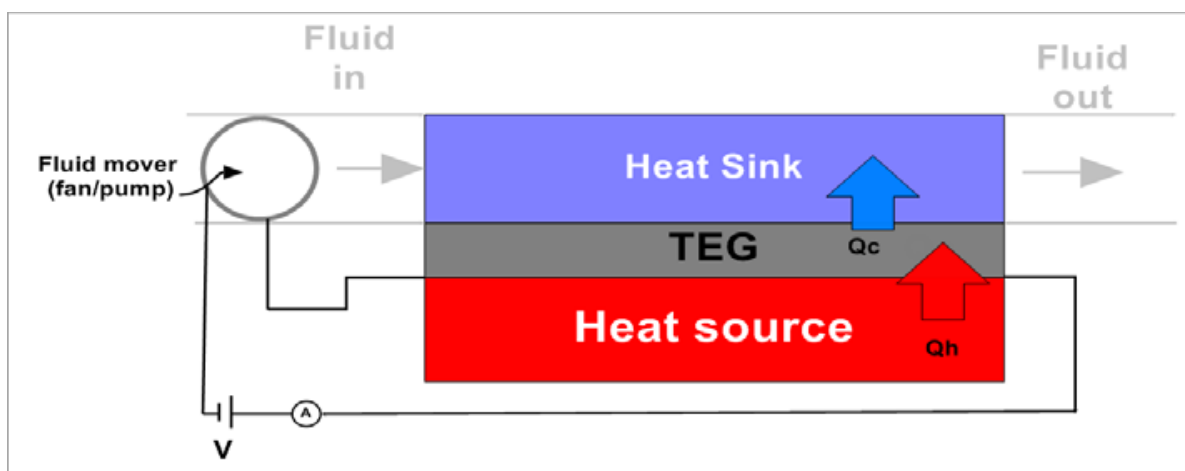

Fig. 1-6.TEG based Self-Cooling Concept

The self-cooling system could be thought of as forced convection cooling system without an external power supply. Thus, the self-cooling system fills the gap between natural convection and forced convection from external power sources. 
It is generally a useful cooling mechanism for applications, where the system (device) may not be adequately cooled by natural convection. In addition, it is also useful for replacing external power sources, where external power sources are not available or feasible.

Typical application areas:

$\checkmark$ Remote area devices

$\checkmark$ Autonomous data acquisition devices

$\checkmark$ Devices with intermittent heating and cooling, where a dedicated cooling system may not be feasible. The self-cooling system allows for the cooling system to kick-in only as needed.

$\checkmark$ Systems where an external power source is not feasible or practical.

$\checkmark$ Back-up systems where an external power source fails or the battery runs out.

There are two main objectives in the thermoelectric generation-based selfcooling concept. The first one is to keep the device at an optimum operating temperature range $\left(\Delta \mathrm{T}_{\mathrm{opt}}\right)$, as prescribed for the proper functioning of the device in a certain environment. The second objective is to generate enough power to run a cooling medium inside the cold-side heat sink. These two objectives are not necessarily mutually inclusive..

For a certain heat input $\mathrm{Q}_{\mathrm{j}}$, there is a maximum allowable device temperature 
$\left(T_{d, \max }\right)$ and corresponding thermal resistance $\left(R_{t h, \max }\right)$ that enables the temperature to be controlled below $\mathrm{T}_{\mathrm{d} \text {,max. }}$.

Despite the huge significance of energy-saving measures for cooling electronic devices and parts, such as microprocessors, there has been very limited research on the concept of heat-driven self-cooling of electronic devices. Solbrekken et al [35]) modeled and demonstrated the application of forced convection cooling of a personal computer microprocessor using power generated by TEG. The same authors [36] also discussed the concept of heatdriven cooling for portable electronics and their results showed that it would be possible to convectively cool an electronic chip using a low-voltage fan.

Martinez et al. [37] also performed an experimental demonstration and numerical study of TEG driven self-cooling configuration. In their experiments, an array of TEG modules were placed directly on top of the heat source plate, which enables the application of a more uniform and maximum temperature at the hot side of the TEG modules. The same authors [23] also presented a dynamic model based on their experimental model.

Zhou et al. [38] studied the effectiveness of harvesting thermoelectric energy from waste heat in Pentium III microprocessors using a commercial TEG module. Gould et al. [39] applied micro-power generation in a standard personal computer using the thermoelectric module. Kiflemariam et al. [40] [41] [42] simulated and 
conducted a parametric study of thermoelectric generator-based self-cooling of devices.

\subsection{Objectives of the Research}

The concept of TEG based self-cooling has not been systematically studied except for few exploratory studies. Thus, the general objective of the PhD thesis work is to develop systematic and comprehensive analytical and numerical models of TEG-based self-cooling systems and validate the results with experimental work. The general objective is achieved through the specific objectives of:

$\checkmark$ Develop a first and second principles-based finite time thermodynamics model of self-cooling systems from basic units' thermoelectric couples to an array of TEG modules.

$\checkmark$ Develop a program based on the thermodynamic principles, which aids in developing a self-cooling system in a given hot side and cold heat sink environment. The program helps set the limits of several parameters of the self-cooling application and is useful in the preliminary design and analysis stage.

$\checkmark$ Conduct an experimental study to validate numerical models as well as study the performance of the self-cooling system.

$\checkmark$ Develop a general numerical methodology to systematically integrate three dimensional computational fluid dynamics (CFD) software with one 
dimensional analysis. This enables detailed design and complex geometrical configuration studies. A parametrical study has been conducted based on the numerical model.

$\checkmark$ Analyze an application of self-cooling liquid cooling-based systems using numerical methods.

$\checkmark$ Develop a dynamic model of self-cooling systems suited for analysis in control system and circuit analysis software, such as DYMOLA, MATLAB/Simulink. The model is implemented in MATLAB/Simulink.

\subsection{Thesis Structure}

- Chapter 2 outlines finite time thermodynamics model of self-cooling system. A program written based on the concepts of chapter 2 is given in Appendix A.

- Chapter 3 demonstrates the experimental procedures and results, which are used to validate numerical methods, and describes the performance of the system.

- Chapter 4 describes a numerical methodology, which could be used for detailed study of self-cooling systems. The methodology is demonstrated by integrating one dimensional models and user-defined functions and equations into existing CFD packages. The numerical results have been extensively validated using experimental data from chapter 4. 
- Chapter 5 shows the application of numerical methods to study liquid based self-cooling systems.

- Chapter 6 outlines a dynamic modeling and analysis of self-cooling system, which is suited for integration in control systems. The model has been validated using experimental data.

\subsection{Original contribution}

The original contribution from this study has been outlined below, which has been included in the thesis and been published in peer-reviewed journal publications and conference proceedings.

- A first principles-based theoretical model of a thermoelectric generationbased self-cooling system (Chapter 2)

- A computer program, which analyzes the feasibility of a self-cooling system and outputs the values and limits of all pertinent parameters (Chapter 2 and Appendix A)

- Perform an experimental study on different configurations of a TEG-based self-cooling system (Chapter 3)

- A numerical methodology to analyze a self-cooling system numerically (Chapter 4)

- Demonstrate the application of numerical methods in popular CFD packages (Chapter 4 and Appendix B) 
- Demonstrate the feasibility of the application of a self-cooling system for a liquid based system (Chapter 5)

- Develop and demonstrate a dynamic model of a self-cooling system suited for control system integration (Chapter 6) 


\section{THERMODYNAMIC ANALYSIS OF SELF-COOLING SYSTEM}

\subsection{Introduction}

In this chapter, TEG based self-cooling system is studied using basic thermodynamic principles. The first law of thermodynamic which is based on the law of conservation of energy is applied to study the energy exchange of the system within the components and outside environment. In addition, second law of thermodynamic is applied to investigate the performance limits or bottlenecks in the application of TEG based self-cooling systems.

The first and second laws of thermodynamics provide basic tools to study a performance of heat engines[43]. Complex thermal systems could be studied by simplifying the system into an equivalent heat engine working between a certain temperature difference defined by heat source and heat sink. The simplified

analysis of the system provide crucial information which helps determine the range of performance of the system before detailed analysis is made.

In classical thermodynamics, the reversible classical heat engines (Figure 2-1) producing a new work of $\mathrm{W}$ and having a heat input of $\mathrm{Q}_{1}$ are assumed to have an efficiency of:

$$
\eta=\frac{W}{Q_{1}}
$$


Or in terms of Carnot efficiency, $\eta_{c}$

$$
\eta_{c}=1-\frac{T_{2}}{T_{1}}
$$

where $T_{2}$ and $T_{1}$ are the temperature of cold and hot reservoirs. However, the idealized form of Carnot heat engine is far from realistic operation of engines. In addition, the conditions of infinite time needed for reversible heat exchange with the environment makes the system impractical for engines which have to provide power.

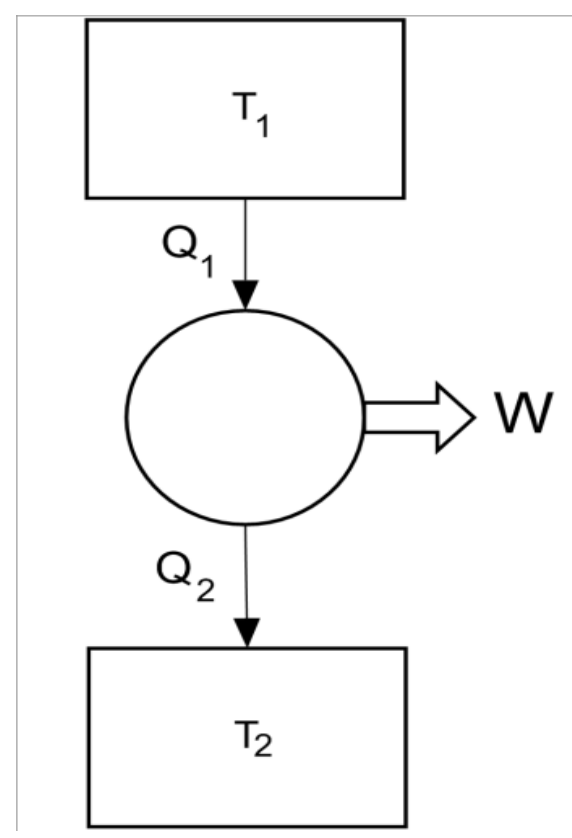

Figure 2-1 Carnot heat engine

Thus, to analyze a system which delivers work at finite time (power), finite time thermodynamic principles are used. The principle of finite time thermodynamics is based on the fact that there is a need for finite temperature difference between 
working fluid and substance or source/ heat sink it is in contact with such that finite amount of heat is transferred in finite time. One of the earliest and important works that deals with finite time thermodynamics is by Curzon and Ahlborn [44]. The studies in finite time thermodynamics were mainly on endoreversible heat engines which are internally reversible but externally irreversible heat engines[45]. The irreversibility is assumed to arise from the heat exchange with the surrounding environment. In this analysis, irreversibilities associated with friction, heat leaks, turbulence and so on are not considered. The external irreversibility due to resistance to the heat flow is considered as shown in Figure 2-2.

The heat engine has hot side and cold side thermal resistances of $\Theta_{1}$ and $\Theta_{2}$ respectively. Curzon and Ahlborn[44] mathematically analyzed the efficiency of endoreversible heat engine operating to be:

$$
\eta_{c, \text { endo }}=1-\left(\frac{T_{2}}{T_{1}}\right)^{n}
$$

Where $\eta_{c, \text { endo is }}$ Carnot efficiency for endoreversible engine. They also determined that maximum power is transferred when $n=1 / 2$. Many studies have then been conducted applying finite time thermodynamic principles to endoreversible heat engines[46][47][48][49][50][51][52]. 


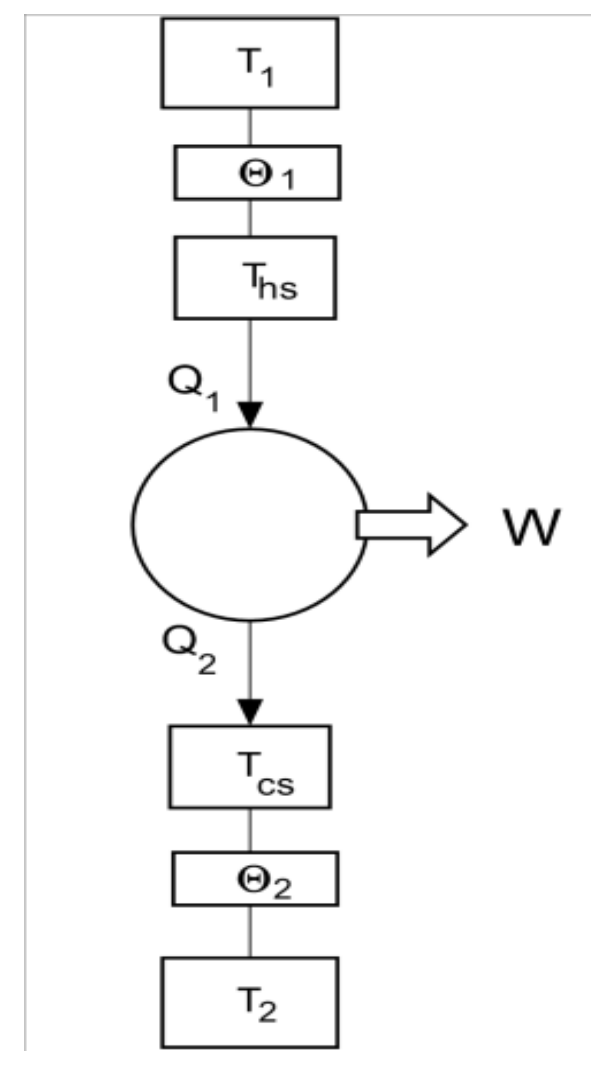

Figure 2-2 endoreversible heat engine

The overall thermal resistances at the hot side and cold side are given by $\Theta_{1}$ and $\Theta_{2}$ respectively. The overall heat transfer coefficients at the hot side (UA) HX and cold side (UA)cx are the reciprocals of $\Theta_{1}$ and $\Theta_{2}$ respectively. In endoreversible heat engine, external irreversibilities associated with cold side and hot side heat exchangers are considered. There have been different models suggested to describe endoreversible heat engines. One of the models was proposed by Gordon[53] in which infinite heat capacity heat reservoirs are assumed.

For the hot side: 


$$
Q_{H}=(U A)_{H X}\left(T_{d}^{n}-T_{h s}^{n}\right)
$$

For the cold side:

$$
Q_{C}=(U A)_{C X}\left(T_{c s}^{n}-T_{f}^{n}\right)
$$

The model assumes non-linear functions of temperature difference. When $n=1$, it coincides with the relation given by Curzon and Ahlborn[44]. When $\mathrm{n}$ approaches infinity, the solution represents Carnot formula. De Vos [45] have suggested $n$ values for different cases such as radiative heat transfer where $n=4$.

Lee and Kim[54] and $\mathrm{Wu}$ [55] have modified the endoreversible model considering finite capacity heat reservoirs. The derivations for the equations are given in the respective papers. The equations for the maximum power conditions are given as:

The hot side temperature of TEG module is given by:

$$
T_{h s}=\left[\frac{\left((U A)_{H X} T_{d}\right)^{0.5}+\left((U A)_{C X} T_{f}\right)^{0.5}}{\left((U A)_{H X}\right)^{0.5}+\left((U A)_{C X}\right)^{0.5}}\right]\left(T_{d}\right)^{0.5}
$$


The cold side temperature of TEG module can be written as:

$$
\begin{aligned}
& T_{c s}=\left[\frac{\left((U A)_{H X} T_{d}\right)^{0.5}+\left((U A)_{C X} T_{f}\right)^{0.5}}{\left((U A)_{H X}\right)^{0.5}+\left((U A)_{C X}\right)^{0.5}}\right]\left(T_{f}\right)^{0.5} \\
& \eta_{c, \text { endo }}=1-\frac{T_{c S}}{T_{h s}} \\
& Q_{H}=(U A)_{H X}\left(T_{d}-T_{h s}\right)
\end{aligned}
$$

However, real engines have both internal and external irreversibilities. There are dissipative processes and heat leaks and other irreversibilities in addition to irreversibilities of finite heat transfer as assumed by endoreversible heat engines.

As shown in Figure 2-3, the heat engine has heat leaks between the reservoirs ( $\mathrm{Q}\llcorner, \mathrm{ex})$, internal irreversibilities inside the heat engine and het leak to the cold reservoir $\left(Q_{L, \text { in }}\right)$ in addition to the irreversibilities of heat transfer between the heat engine and reservoirs. Thermoelectric generator based self-cooling system fundamentally consists of TEG module, heat source and cold side heat exchanger. TEG modules are direct conversion heat engines with internal as well as external irreversibilities. 


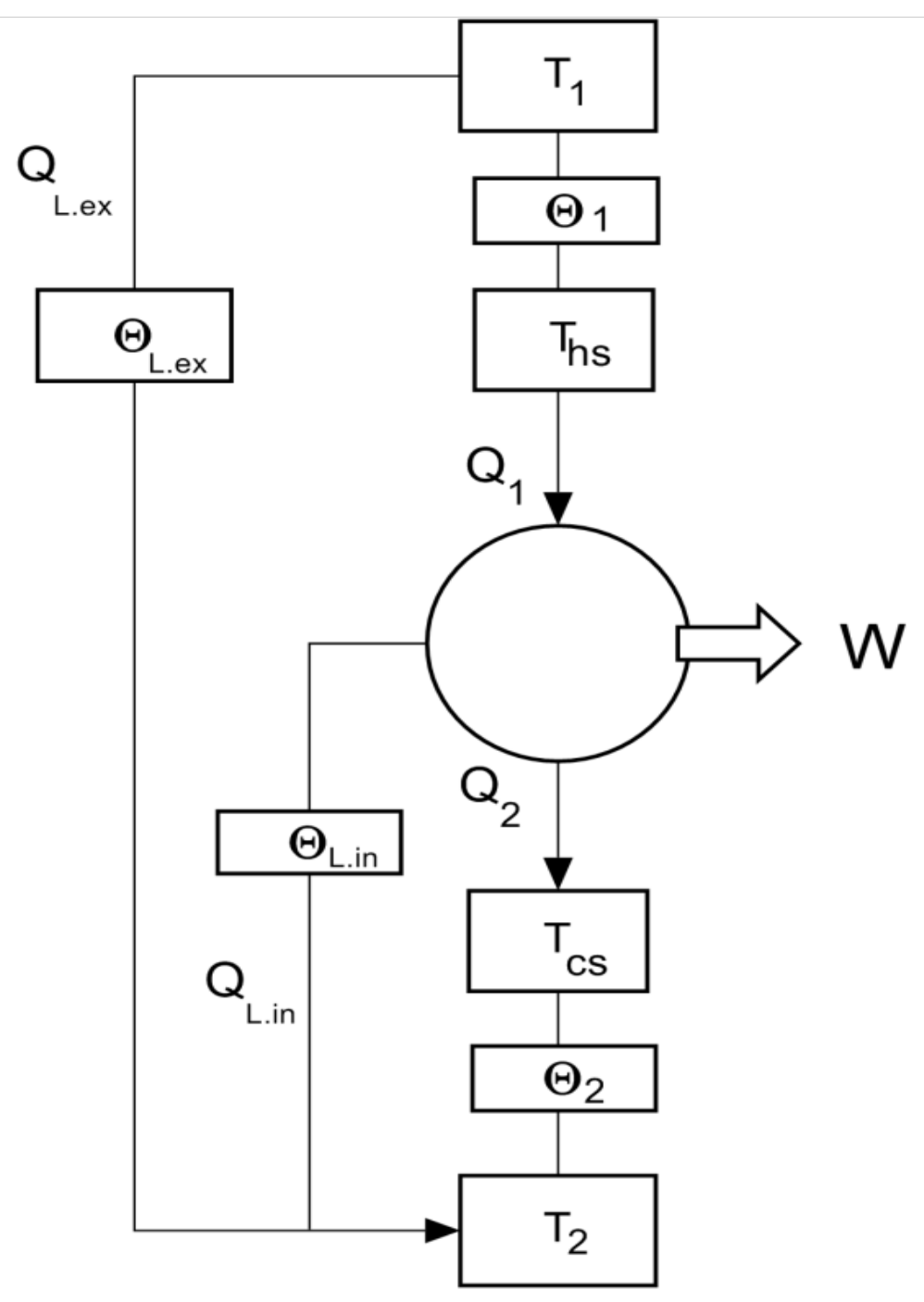

Figure 2-3 Externally and internally irreversible heat engine

There are internal irreversibilities due to joule heating and thermal conduction in addition to heat leak to the cold side reservoirs. In addition, there is heat leak between reservoirs and external irreversibilities for heat exchange between TEG module and reservoirs. Moreover, there are internal irreversibilities in the 
reservoir working fluid. Thus, TEG based self-cooling system could be more realistically modeled as internally and externally irreversible heat engine.

\subsection{Thermoelectric couple governing equations}

A thermoelectric couple could be considered as a fundamental unit of TEG module. It consists of $\mathrm{p}$ and $\mathrm{n}$ legs connected by a conductor.

\subsubsection{Energy balance equations}

The change conservation equation can be written as:

$$
\nabla \cdot \vec{J}+\frac{\partial \rho}{\partial t}=0
$$

where $\vec{J}$ is the current density.

The heat flux conservation equation is written as

$$
\nabla \cdot q+\rho C_{v} \frac{\partial T}{\partial t}=\dot{g}
$$

Where $\nabla \cdot \mathrm{q}$ consists of Peltier heat and the heat conducted from hot side to the cold side of the leg and $\dot{g}$ represents the internal heat generation due to Joule and Thomson heat effects. Eq. 2.2.2 can be rewritten as:

$$
\nabla \cdot(\pi J-k \nabla T)+\rho C_{v} \frac{\partial T}{\partial t}=E \cdot J
$$


But $J$ is given by:

$$
J=\sigma E-\sigma(\alpha \nabla T)
$$

Substituting Eq. 2.2.4 in Eq. 2.2.3:

$$
\nabla \cdot(\pi J-k \nabla T)+\rho C_{v} \frac{\partial T}{\partial t}=\sigma E^{2}-\sigma(\alpha \nabla T) E
$$

And the electrical conductivity of materials $(\sigma(T))$ can be expressed as:

$$
\sigma(T)=\frac{J}{E}
$$

And the Seebeck Coefficient $\alpha(T)$ :

$$
\alpha(T)=\frac{\pi(T)}{T}
$$

Substituting for Seebeck coefficient:

$$
\begin{aligned}
& \nabla \cdot(\alpha T J-k \nabla T)+\rho C_{v} \frac{\partial T}{\partial t}=\sigma E^{2}-\sigma(\alpha \nabla T) E \\
& \rho C_{v} \frac{\partial T}{\partial t}=\nabla \cdot(\alpha T J)+\nabla \cdot(k \nabla T)+\frac{J^{2}}{\sigma}-\left(T \frac{\partial \alpha}{\partial T} \nabla T\right) J \quad 2.2 .9
\end{aligned}
$$


For thermoelectric modules, it can be assumed that a one-dimensional energy balance equation could be used to represent the heat flows inside the $p$ and $n$ legs:

$$
\begin{aligned}
& 0 \leq y \leq l_{p} \\
& 0 \leq y \leq l_{n}
\end{aligned}
$$

Where $I_{p}$ and $I_{n}$ are the lengths of $p$ and $n$ legs.

$$
\rho C_{v} \frac{\partial T(y, t)}{\partial t}=\frac{\partial \alpha}{\partial y} T(y, t) J+k \frac{\partial^{2} T(x, t)}{\partial x^{2}}+\frac{J^{2}}{\sigma}-T(x, t) \frac{\partial \alpha}{\partial T} J \frac{\partial T(x, t)}{\partial x}
$$

The general equation could be approximated with several assumptions.

a) If the temperature difference across the elements is not large, the value of $\mathrm{T}(\mathrm{x}, \mathrm{t}) \partial \alpha / \partial T$ could be neglected.

b) The variation of seebeck coefficient with position $\partial \alpha / \partial y$ can be neglected with isotropic property assumption.

$$
k \frac{\partial^{2} T(x, t)}{\partial x^{2}}=-\frac{J^{2}}{\sigma}
$$

The boundary conditions at the ends of thermodynamic legs are: 


$$
\begin{aligned}
& T(y=0)=T_{h} \\
& T\left(y=l_{p} / l_{n}\right)=T_{c}
\end{aligned}
$$

Integrating the equation and applying the boundary conditions:

$$
\frac{\partial^{2} T(y, t)}{\partial x^{2}}=-\frac{J^{2}}{\sigma k}
$$

Integrating twice results in equations with unknown constant $C_{1}$ and $C_{2}$ :

$$
\begin{aligned}
& \frac{\partial T(y, t)}{\partial x}=-\frac{J^{2}}{\sigma k} y+C_{1} \\
& T(y, t)=-\frac{J^{2}}{\sigma k} \frac{y^{2}}{2}+C_{1} y+C_{2}
\end{aligned}
$$

Applying boundary equations and solving for $\mathrm{C}_{2}$ :

$$
T_{h}=C_{2}
$$




$$
T_{c}=-\frac{J^{2}}{\sigma k} \frac{l^{2}}{2}+C_{1} l+T_{h}
$$

The value of $C_{1}$ is given by:

$$
\begin{aligned}
& C_{1}=\frac{T_{c}-T_{h}}{l}+\frac{J^{2}}{\sigma k} \frac{l}{2} \\
& \frac{\partial T(y, t)}{\partial x}=-\frac{J^{2}}{\sigma k} y-\frac{T_{h}-T_{c}}{l}+\frac{J^{2}}{\sigma k} \frac{l}{2}
\end{aligned}
$$

The variation of temperature could then be expressed as:

$$
\begin{aligned}
& T(y, t)=T_{h}-\left(\frac{T_{h}-T_{c}}{l}-\frac{J^{2}}{\sigma k} \frac{l}{2}\right) y-\frac{J^{2}}{\sigma k} \frac{y^{2}}{2} \\
& T(y, t)=T_{h}-\frac{\left(T_{h}-T_{c}\right)}{l} y+\frac{J^{2}}{\sigma k} \frac{l}{2} y-\frac{J^{2}}{\sigma k} \frac{y^{2}}{2} \\
& T(y, t)=T_{h}-\frac{\left(T_{h}-T_{c}\right)}{l} y+\frac{J^{2}}{2 \sigma k}\left(y l-y^{2}\right)
\end{aligned}
$$




\subsubsection{Entropy balance equations}

The rate of change of internal energy is given as:

$$
\begin{aligned}
& \frac{\partial u}{\partial t}=C_{v} \frac{\partial T}{\partial t} \\
& \rho \frac{\partial u}{\partial t}=\nabla \cdot(\alpha T J)+\nabla \cdot(k \nabla T)+\frac{J^{2}}{\sigma}-\left(T \frac{\partial \alpha}{\partial T} \nabla T\right) J
\end{aligned}
$$

Using the Gibbs free energy relation between internal energy and entropy flux, s

$$
\partial u=-T \partial s
$$

$$
\rho \frac{(T \partial s)}{\partial t}=\nabla \cdot(\alpha T J)+\nabla \cdot(k \nabla T)+\frac{J^{2}}{\sigma}-\left(T \frac{\partial \alpha}{\partial T} \nabla T\right) J \quad 2.2 .29
$$

$$
\rho \frac{\partial s}{\partial t}=\nabla \alpha \cdot J+\frac{\nabla \cdot(k \nabla T)}{T}+\frac{J^{2}}{T \sigma}-\left(\frac{\partial \alpha}{\partial T} \nabla T\right) J
$$

$$
\frac{\nabla \cdot(k \nabla T)}{T}=\nabla \cdot\left(\frac{k \nabla T}{T}\right)-k \nabla T \cdot \nabla\left(\frac{1}{T}\right)
$$

$$
\rho \frac{\partial s}{\partial t}=\nabla \alpha \cdot J+\nabla \cdot\left(\frac{k \nabla T}{T}\right)-k \nabla T \cdot \nabla\left(\frac{1}{T}\right)+\frac{J^{2}}{T \sigma}-\left(\frac{\partial \alpha}{\partial T} \nabla T\right) J \quad 2.2 .32
$$


The first terms in indicate entropy terms due to heat conduction and peltier heat which is expressed as entropy flux $\mathrm{J}^{\mathrm{s}}$

$$
J^{s}=\alpha \cdot J+\nabla \cdot\left(\frac{k \nabla T}{T}\right)
$$

The other terms are associated with irreversibility in the thermoelectric modules. These include internal heat losses, Joule heat and Thomson heat losses.

$$
\dot{S}_{T E G}=-k \nabla T \cdot \nabla\left(\frac{1}{T}\right)+\frac{J^{2}}{T \sigma}-\left(\frac{\partial \alpha}{\partial T} \nabla T\right) J
$$

According to the assumption of one dimensional analysis in the y direction:

For the p-leg

$$
\begin{aligned}
& J^{s}(y)=\alpha \cdot J+\frac{k}{T}\left(-\frac{J^{2}}{\sigma k} y-\frac{T_{h}-T_{c}}{l}+\frac{J^{2}}{\sigma k} \frac{l_{p, n}}{2}\right) \\
& J^{s}(y)_{-} p=\alpha_{p} \cdot J+\frac{J^{2}}{\sigma T(y)}\left(y-\frac{l_{p, n}}{2}\right)+\frac{T_{h}-T_{c}}{l}
\end{aligned}
$$

For the n-leg 


$$
J^{s}(y)_{-} n=-\alpha_{n} \cdot J-\frac{J^{2}}{\sigma T(y)}\left(y-\frac{l_{p, n}}{2}\right)-\frac{T_{h}-T_{c}}{l}
$$

2.2.3. Heat transfer for thermoelectric couple

The heat transfer at the hot side of thermoelectric couple is given as:

$$
\begin{aligned}
& q_{h}=T_{h} \cdot\left(J^{s}(0)_{-} p-J^{s}\left(l_{p, n}\right)_{-} n\right) \\
& q_{h}=\alpha_{p}-\left(-\alpha_{n}\right) \cdot J T_{h}+2 k \frac{T_{h}-T_{c}}{l_{p, n}}-\frac{J^{2}}{\sigma} l_{p, n}
\end{aligned}
$$

The heat transfer at the cold side of thermoelectric couple is expressed as:

$$
\begin{aligned}
& q_{c}=T_{c} \cdot\left(J^{s}\left(l_{p, n}\right)_{-} p-J^{s}(0)_{-} n\right) \\
& q_{c}=\alpha_{p}-\left(-\alpha_{n}\right) \cdot J T_{c}+2 k \frac{T_{h}-T_{c}}{l_{p, n}}+\frac{J^{2}}{\sigma} l_{p, n}
\end{aligned}
$$

The Seebeck coefficient for a thermoelectric couple $\alpha_{\mathrm{p}, \mathrm{n}}$ is defined as:

$$
\alpha_{p n}=\alpha_{p}-\left(-\alpha_{n}\right)
$$




$$
\begin{aligned}
& q_{h}=\alpha_{p n} \cdot J T_{h}+2 k \frac{T_{h}-T_{c}}{l_{p, n}}-\frac{J^{2}}{\sigma} l_{p, n} \\
& q_{c}=\alpha_{p n} \cdot J T_{c}+2 k \frac{T_{h}-T_{c}}{l_{p, n}}+\frac{J^{2}}{\sigma} l_{p, n}
\end{aligned}
$$

For thermoelectric pairs with dissimilar conductivity $k_{p}$ and $k_{n}$ :

$$
\begin{aligned}
& q_{h}=\alpha_{p n} \cdot J T_{h}+\left(k_{p}+k_{n}\right) \frac{T_{h}-T_{c}}{l_{p, n}}-\frac{J^{2}}{\sigma} l_{p, n} \\
& q_{c}=\alpha_{p n} \cdot J T_{c}+\left(k_{p}+k_{n}\right) \frac{T_{h}-T_{c}}{l_{p, n}}+\frac{J^{2}}{\sigma} l_{p, n}
\end{aligned}
$$

The power produced $P_{\text {gen }}$ per area is the difference between $q_{h}$ and $q_{c}$ :

$$
P_{g e n}=q_{h}-q_{c}=\alpha_{p, n} J\left(T_{h}-T_{c}\right)-\frac{J^{2}}{\sigma} l_{p, n}
$$

The efficiency of thermoelectric generator, $\eta_{g}$ is given as the ratio $\mathrm{Pgen}_{\mathrm{g}} / \mathrm{q}_{\mathrm{h}}$ : 


$$
\eta_{g}=\frac{\alpha_{p, n} J\left(T_{h}-T_{c}\right)-\frac{J^{2}}{\sigma} l_{p, n}}{\alpha_{p, n} \cdot J T_{h}+2 k \frac{T_{h}-T_{c}}{l_{p, n}}-\frac{J^{2}}{\sigma} l_{p, n}}
$$

\subsection{Governing equations for TEG module}

A TEG module is formed by connection of number of thermoelectric pairs connected in series electrically and in parallel thermally. For a number of thermoelectric pairs, $n_{p}$, the thermal conductance can be defined as:

$$
\begin{aligned}
& K=n_{p}\left(\frac{k_{p} A_{p}}{l_{p, n}}+\frac{k_{n} A_{n}}{l_{p, n}}\right) \\
& R_{T E G}=n_{p}\left(\frac{l_{p, n}}{A_{p} \sigma_{p}}+\frac{l_{p, n}}{A_{n} \sigma_{n}}\right) \\
& \alpha_{m}=n_{p}\left(\alpha_{p n}\right)
\end{aligned}
$$

And defining the average temperature of the hot side and cold side of TEG module as: $T_{h s}$ and $T_{c s}$ :

$$
q_{h}=\alpha_{m} \cdot I T_{h s}+K\left(T_{h s}-T_{c s}\right)-0.5 R_{T E G} I^{2}
$$




$$
\begin{aligned}
& q_{c}=\alpha_{m} \cdot I T_{c s}+K\left(T_{h s}-T_{c s}\right)+0.5 R_{T E G} I^{2} \\
& P_{g e n}=\alpha_{m} \cdot I\left(T_{h s}-T_{c s}\right)-R_{T E G} I^{2}
\end{aligned}
$$

The maximum generated power could be determined by derivation of Eq.2.3.6 with respect to I:

$$
\frac{d P_{g e n}}{d I}=\alpha_{m}\left(T_{h s}-T_{c s}\right)-2 R_{T E G} I
$$

By setting the equation to zero, current at maximum power, $I_{p, \text { max }}$ could be determined:

$$
I_{p, \max }=\frac{\alpha_{m}\left(T_{h s}-T_{c s}\right)}{2 R_{T E G}}
$$

The open circuit voltage is voltage produced by TEG module when no current is flowing (open circuit) and is given by:

$$
V_{o c}=\alpha_{m}\left(T_{h s}-T_{c s}\right)
$$


The voltage at maximum power, $\mathrm{V}_{\mathrm{p}, \max }$ is:

$$
V_{p, \max }=\frac{V_{o c}}{2}
$$

The current in a TEG module, I is given as:

$$
I=\frac{\alpha_{m}\left(T_{h s}-T_{c s}\right)}{R_{T E G}+R_{L}}
$$

Defining the ratio between $R_{L}$ and $R_{T E G}$ to be $R_{L} / R_{T E G}=\varsigma$ :

$$
I=\frac{\alpha_{m}\left(T_{h s}-T_{c s}\right)}{R_{T E G}(1+\varsigma)}
$$

The maximum power from TEG module could thus be derived as:

$$
\begin{aligned}
& P_{g e n, \max }=\alpha_{m} \cdot I_{p, \max }\left(T_{h s}-T_{c s}\right)-R_{T E G} I_{p, \max }^{2} \\
& P_{g e n, \max }=\frac{\alpha_{m}^{2}\left(T_{h s}-T_{c s}\right)^{2}}{4 R_{T E G}}
\end{aligned}
$$




\subsection{TEG module with hot and cold reservoir}

The heat transfer inside the TEG modules at the hot side could be described as:

$$
Q_{h}=I T_{h s} \alpha_{m}+\frac{\left(T_{h s}-T_{c s}\right)}{\Theta_{T E G}}-\frac{R_{T E G}}{2} I^{2}
$$

And at the cold side of the modules:

$$
Q_{c}=I T_{c s} \alpha_{m}+\frac{\left(T_{h s}-T_{c s}\right)}{\Theta_{T E G}}+\frac{R_{T E G}}{2} I^{2}
$$

The heat transfer at the hot side can be modeled as:

$$
Q_{H X}=U_{H X} A_{H X}\left(T_{d}-T_{h s}\right)
$$

where $U_{H X}$ is the overall heat transfer coefficient between the hot side of TEG module and heat source at temperature of $T_{d} . A_{H x}$ is the area of heat exchanger between TEG module and heat source.

Similarly, the heat transfer the cold side also be expressed as:

$$
Q_{C X}=U_{C X} A_{C X}\left(T_{c s}-T_{f}\right)
$$


where $U_{c x}$ is the overall heat transfer coefficient between the hot side of TEG module and heat sink at temperature of $T_{f}$. Acx is the area of heat exchanger between TEG module and heat sink.

For a condition where all the heat from device is transferred into the thermoelectric generator module, it can be assumed that $Q_{H X}=q_{h}$ :

$$
\begin{aligned}
& Q_{h}=Q_{H X}=I T_{h s} \alpha_{m}+\frac{\left(T_{h s}-T_{c s}\right)}{\Theta_{T E G}}-\frac{R_{T E G}}{2} I^{2}=U_{H X} A_{H X}\left(T_{d}-T_{h s}\right) \quad 2.4 .5 \\
& T_{h s}=\frac{U_{H X} A_{H X} T_{d}-\frac{\left(T_{h s}-T_{c s}\right)}{\Theta_{T E G}}+\frac{R_{T E G}}{2} I^{2}}{U_{H X} A_{H X}+\alpha_{m} I}
\end{aligned}
$$

Similarly for a condition where all the heat into rejected to the heat sink, it can be assumed that

$$
\begin{gathered}
Q_{C}=Q_{C X}=I T_{c s} \alpha_{m}+\frac{\left(T_{h s}-T_{c s}\right)}{\Theta_{T E G}}+\frac{R_{T E G}}{2} I^{2}=U_{C X} A_{C X}\left(T_{c s}-T_{f}\right) \\
T_{c s}=\frac{U_{C X} A_{C X} T_{f}+\frac{\left(T_{h s}-T_{c s}\right)}{\Theta_{T E G}}+\frac{R_{T E G}}{2} I^{2}}{U_{C X} A_{C X}-\alpha_{m} I}
\end{gathered}
$$




\subsection{Analysis of performance of TEG based self-cooling system}

The performance of the system including determining the maximum amount heat that could be transferred between the heat source device at fixed temperature of $T_{d}$ and the heat sink temperature of $T_{f}$ is analyzed. Thus, the simultaneous solution of Eq. 2.3.11, Eq. 2.4.6 and Eq. 2.4.8 yields the values of heat transfer, temperature at the TEG modules, voltage, current and power from TEG module.

Rewriting the equations on a thermocouple basis:

$$
\begin{aligned}
& \left((U A)_{H X}+K_{p n}\right) T_{h s}+\alpha_{p n} I T_{h s}-(U A)_{H X} T_{d}-K_{p n} T_{c s}-0.5 R_{p n} I^{2}=0 \quad 2.5 .1 \\
& \left((U A)_{C X}+K_{p n}\right) T_{c s}-\alpha_{p n} I T_{c s}-(U A)_{C X} T_{f}-K_{p n} T_{h s}-0.5 R_{p n} I^{2}=0 \quad 2.5 .2
\end{aligned}
$$

Rewriting the equations on a TEG module basis:

$$
\begin{gathered}
\left((U A)_{H X}+K_{T E G}\right) T_{h s}+\alpha_{m} I T_{h s}-(U A)_{H X} T_{d}-K_{T E G} T_{c s}-0.5 R_{T E G} I^{2}=0 \quad 2.5 .3 \\
\left((U A)_{C X}+K_{T E G}\right) T_{c S}-\alpha_{m} I T_{c s}-(U A)_{C X} T_{f}-K_{T E G} T_{h s}-0.5 R_{T E G} I^{2}=0 \quad 2.5 .4
\end{gathered}
$$




\subsubsection{Overall heat transfer coefficient on hot side}

The overall heat transfer coefficient the hot side $(U A) \mathrm{Hx}$ is given by the sum resistances between the heat source device and hot side of the thermoelectric generator module.

$$
(U A)_{H X}=U_{H} A_{H}=\frac{1}{\Theta_{H X}}
$$

where $\Theta_{\mathrm{HX}}$ is the overall thermal resistance on the hot side. The overall thermal resistance on the hot side $\left(\Theta_{\mathrm{HX}}\right)$ consists of material thermal resistance inside the device and interface thermal resistance between device and TEG module. Depending on the relative area of the device to the spreader, constriction or spreading thermal resistance heat spreader and material heat spreader may also account for the total thermal resistance. If the device and/or the spreader have exposed area to the environment, convection resistance could also exist.

In this section, for the sake of brevity in the fundamental thermodynamic analysis of self-cooling system, the device is assumed to have same area the TEG module. It is also assumed that primary heat path is conduction to the TEG module and convection heat transfer to the surrounding is neglected.

The material thermal resistance of the device is expressed as: 


$$
\Theta_{d}=\frac{t_{d}}{k_{d} A_{d}}
$$

\subsubsection{Overall heat transfer coefficient on cold side}

The overall heat transfer coefficient the cold side (UA)cx is given by the sum resistances between the cold side of TEG module and cold reservoir (heat sink).

$$
(U A)_{C X}=U_{C} A_{C}=\frac{1}{\Theta_{C X}}
$$

where $\Theta_{c x}$ is the overall thermal resistance on the cold side. For the fundamental thermodynamic analysis, $\Theta_{c x}$ consists of spreading thermal resistance from cold side of TEG module to the base of heat sink $\left(\Theta_{\mathrm{sp}}\right)$, material thermal resistance inside the cold side heat exchanger $\left(\Theta_{b}\right)$ and convection heat coefficient from the heat exchanger to heat sink $\left(\Theta_{\text {conv }}\right)$.

The spreading thermal resistance $\Theta_{\text {sp }}$ from smaller area $\left(A_{1}\right)$ to the bigger area $\left(A_{2}\right)$ could be calculated using the equation [56]:

$$
\Theta_{s p}=\Theta_{s p, b}-\Theta_{s p, a}
$$

where: 


$$
\begin{aligned}
& \Theta_{s p, a}=\frac{\sqrt{A_{2}}-\sqrt{A_{1}}}{k_{b} \sqrt{\pi A_{1} A_{2}}} \Omega \\
& \Omega=\frac{\lambda k_{2} A_{2} R_{\text {conv }}+\tanh \left(\lambda t_{2}\right)}{1+\lambda k_{2} A_{2} R_{\text {conv }} \tanh \left(\lambda t_{2}\right)} \\
& \lambda=\frac{\pi^{3 / 2}}{\sqrt{A_{2}}}+\frac{1}{\sqrt{A_{1}}} \\
& \Theta_{s p, b}=\frac{1}{2 k_{b} \sqrt{A_{1}}}\left(1-\frac{\sqrt{A_{1}}}{\sqrt{A_{2}}}\right)^{3 / 2} \Omega
\end{aligned}
$$

The material thermal resistance of the base of cold side heat exchanger is expressed as:

$$
\Theta_{b, c x}=\frac{t_{b, c x}}{k_{b, c x} A_{b, c x}}
$$

The convection heat transfer from the surfaces of cold side heat exchanger to the heat sink can be expressed as: 


$$
\Theta_{\text {conv }}=\frac{1}{h A_{c x}}
$$

Where $\mathrm{h}$ is the convection heat transfer coefficient and $A_{c x}$ is the effective area of cold side heat exchanger.

In the present analysis, parallel plate-fin heat exchanger is considered as it is commonly used of heat exchanger for air-side heat exchangers. The effective area of cold-side heat exchanger is given as:

$$
A_{c x}=A_{\text {base }}+N_{f} \eta_{f} A_{f}
$$

where $N_{\text {fin }}$ is the number of fins, $\eta_{f}$ is the fin efficiency, $A_{\text {base }}$ is the exposed area of the heat exchanger and $A_{f}$ is area of a single fin:

$$
A_{\text {base }}=\left(N_{f}-1\right) b L
$$

$$
\begin{aligned}
& A_{f}=2 H_{f} L \\
& n_{f}=\frac{\tanh \left(m H_{f}\right)}{m H_{f}}
\end{aligned}
$$

where $m$ is expressed as: 


$$
m=\sqrt{\frac{2 h}{k_{b} t_{f}}}
$$

where $k_{b}$ is the thermal conductivity of the fin material.

The convection heat transfer coefficient for forced flow, $h$ could be estimated by an analytical model for both developing and fully developed laminar flow regimes developed by Teertstra et al[57].

$$
N u_{b}=\left[\frac{1}{\left[\frac{\mathrm{RePr}}{2}\right]^{3}}+\frac{1}{\left[0.644 \sqrt{\operatorname{Re} \operatorname{Pr}^{0.33}} \sqrt{1+\frac{3.65}{\sqrt{\mathrm{Re}}}}\right]^{3}}\right]^{-0.33}
$$

The modified Reynolds number, Re is given as:

$$
\operatorname{Re}=\frac{\rho_{f} U b}{\mu_{f}} \frac{b}{L}
$$

Where $\rho_{f}$ and $\mu f$ are the density and dynamic viscosity of the fluid and $U$ is the velocity of fluid.

The Prandtl number, Pr could be expressed as: 


$$
\operatorname{Pr}=\frac{\mu c_{p}}{k_{f}}
$$

Where $\mathrm{k}$ is the thermal conductivity of the fluid

And convection heat transfer coefficient, $\mathrm{h}_{\text {conv }}$ is:

$$
h_{c o n v}=\frac{N u_{b} k}{b}
$$

It is also of interest to investigate the natural convection heat transfer coefficient which allows the comparison of self-cooling system with naturally cooled systems. Culham et al[58] proposed an analytical model to estimate heat transfer coefficient from rectangular heat sinks cooled by natural convection. They proposed a Nusselt number $\left(\mathrm{Nu}_{\varpi}\right)$ based on characteristic length, $\varpi$ which is a square root of total wetted area of the finned heat sink :

$$
N u_{\sigma}=N u_{\varpi}^{\infty}+f(p r) g_{\varpi} R a_{\varpi}^{0.25}
$$

where $N u_{\varpi}^{\infty}$ is diffusive limit of the cuboid which is defined as:

$$
N u_{\sigma}^{\infty}=\frac{3.192+1.868\left(H_{f} / L\right)^{0.76}}{\left.\sqrt{1+1.189\left(H_{f}\right.} / L\right)}
$$


In Eq. 2.5.29 $g_{\varpi}$ and $f(p r)$ are the body gravity function, Prandtl number function respectively which are given by.

$$
\begin{gathered}
g_{\sigma}=2^{1 / 8}\left[\frac{H_{f}\left(n_{f} H_{f}+t_{f}+W\right)^{2}}{\left(n_{f} t_{f} L+t_{f} W+H_{f}\left(n_{f} H_{f}+t_{f}+W\right)\right)^{3 / 2}}\right]^{1 / 4} 2.5 .26 \\
N u_{\sigma}^{\infty}=\frac{3.192+1.868\left(H_{f} / L\right)^{0.76}}{\sqrt{1+1.189\left(H_{f} / L\right)}} \\
f(p r)=\frac{0.670}{\left[1+(0.5 / p r)^{9 / 16}\right]^{4 / 9}}
\end{gathered}
$$

The Rayleigh number is defined as a function of wetted area given as:

$$
R a_{\varpi}=G r \cdot \operatorname{Pr}=\frac{\operatorname{Pr} g \beta\left(T_{s}-T_{\infty}\right) \varpi^{3}}{v^{2}}
$$

Thus, the natural convection coefficient is estimated by

$$
h=\frac{N u_{\varpi} k_{f}}{\varpi}
$$




\subsubsection{Thermal interface resistance $\Theta_{\text {in }}$}

In this section, the thermal contact resistance between surfaces is discussed. For Self-cooling applications, as TEG module is sandwiched between the hot-side and cold-side heat exchangers, it is important to understand the effect of thermal resistance between two conforming surfaces. The total thermal interface resistance is due to the combined effect of micro-contact thermal resistance $\left(\Theta_{c}\right)$ and micro-gap thermal resistance $\left(\Theta_{g}\right)$. Usually thermal interface materials (TIM) are used to reduce the micro-gap thermal resistances by filling out the interstitial air gaps between the surfaces.

The total micro contact resistance $\left(\Theta_{c}\right)$ between two surfaces is given by [59]:

$$
\Theta_{c}=\sum_{j=1}^{n_{c}} \frac{F_{a} k_{s}}{0.565 c_{1}(o / m)}\left(\frac{o}{o_{c} m}\right)^{-c_{2}}
$$

Where for two contacting surfaces represented by subscripts 1 and 2, o is the RMS surface roughness: $o=\sqrt{0^{1}+o^{2}}, \mathrm{~m}$ is the absolute surface slope $\mathrm{m}=$ $\sqrt{\mathrm{m}^{1}+\mathrm{m}^{2}}$ and $\mathrm{ks}$ is described as $\mathrm{k}_{\mathrm{s}}=0.5\left(\mathrm{k}_{1}^{-1}+\mathrm{k}_{2}^{-1}\right) . \mathrm{c} 1$ and $\mathrm{c} 2$ are the applied force on the surfaces, Vickers hardness correlation coefficient of surface 1 and 2 respectively. $\mathrm{Fa}$ is the applied force. The total number of micro contacts $\mathrm{n}_{\mathrm{c}}$ are given by the equation: 


$$
n_{c}=0.0625\left(\frac{m}{o}\right)^{2} \frac{\exp \left(-\mathrm{X}^{2} / o^{2}\right)}{\operatorname{erf}(\mathrm{X} / \sqrt{2 o})} A_{a p p}
$$

Where $A_{p p}$ is the apparent are of contact between the surfaces and the mean plane separation of the surfaces $\mathrm{X}$ is given as:

$$
\mathrm{X}=o \sqrt{2} \operatorname{erfc^{-1}}\left(\frac{2 F_{a}}{A_{n} \vartheta}\right)
$$

Where $\vartheta$ is the micro hardness of softer material and $A_{n}$ is the normal surface where force is applied.

The surface gap heat transfer is modeled as thermal conduction inside the interstitial gas/air [60]. The analysis of thermal conduction in the gas is a complex phenomenon heat flow regimes consisting of temperature jump, temperature slip, free molecule and transition continuum. Song et al [61] presented a simplified kinetic theory model based on Maxwell velocity distribution law. The thermal resistance for the interstitial gap is given as:

$$
\Theta_{g}=\sum_{j=1}^{n_{c}}\left(\frac{k_{g} A_{g}}{\mathrm{X}+\Lambda}\right)^{-1}-
$$

Where $X$ is approximated to represent the mean gap height and $\Lambda$ is given as: 


$$
\Lambda=\left(\frac{w_{2}\left(2-w_{1}\right)+w_{1}\left(2-w_{2}\right)}{w_{1} w_{2}}\right)\left(\frac{2 \gamma}{1+\gamma}\right)\left(\frac{C_{g} T_{g}}{P_{g}}\right)
$$

where $C_{g}, T_{g}, P_{g}$ are the specific gas constant, temperature and pressure of the gas, $\mathrm{Y}$ is the ratio of specific gas constants and $w_{1}$ and $w_{2}$ are the thermal accommodation coefficients for the solid bodies describing their interaction with the gas.

The total thermal interface resistance $\left(\Theta_{i n}\right)$ is thus given as the sum of the resistances:

$$
\Theta_{i n}=\left(\frac{1}{\Theta_{c}}+\frac{1}{\Theta_{g}}\right)^{-1}
$$

\subsubsection{Pressure drop in cold side heat exchanger}

The pressure drop in the cold side heat exchanger is considered for parallel-plate fin heat exchanger which is the most fundamental and widely used type of heat exchanger.

For a pressure drop at the cold side heat exchanger between points 1 and 2: 


$$
\Delta P_{12}=P_{2}-P_{1}
$$

The pressure drop for fully developed flow inside parallel plates could be estimated using the expression from [62] and [63].

$$
\Delta P_{12}=\frac{\rho_{f} U^{2}}{2}\left[K_{c}+4 f \frac{L}{D_{h}}+K_{e}\right]
$$

Where $f$ is a friction factor and $\mathrm{K}_{\mathrm{c}}$ and $\mathrm{K}_{\mathrm{e}}$ are pressure losses associated with contraction and expansion losses respectively. $D_{h}$ is the hydraulic diameter. The values for $\mathrm{K}_{\mathrm{c}}$ and $\mathrm{K}_{\mathrm{e}}$ could be substituted in the previous equation and the resulting expression is:

$$
\Delta P_{12}=\frac{\rho_{f} U^{2}}{2}\left[0.42\left(1-\vartheta^{2}\right)+4 f \frac{L}{D_{h}}+\left(1-\vartheta^{2}\right)^{2}\right]
$$

where $f$ is given by:

$$
f=\frac{\left(24-32,527 \theta+46.721 \theta^{2}-40.829 \theta^{3}+22.954 \theta^{4}-6.089 \theta^{5}\right)}{\operatorname{Re}} \quad 2.5 .40
$$

where 


$$
\theta=\frac{h}{H_{f}}
$$

And

$$
\vartheta=1-\frac{N_{\text {fin }} t_{f}}{W}
$$

Power consumed by a flow mover, $P_{\text {con }}$ to move a fluid from 1 to 2 could be expressed by:

$$
P_{\text {con }}=\Delta P_{12} \cdot \operatorname{Vol}_{12}
$$

where $\mathrm{Vol}_{12}$ is the volume flow rate inside a heat exchanger.

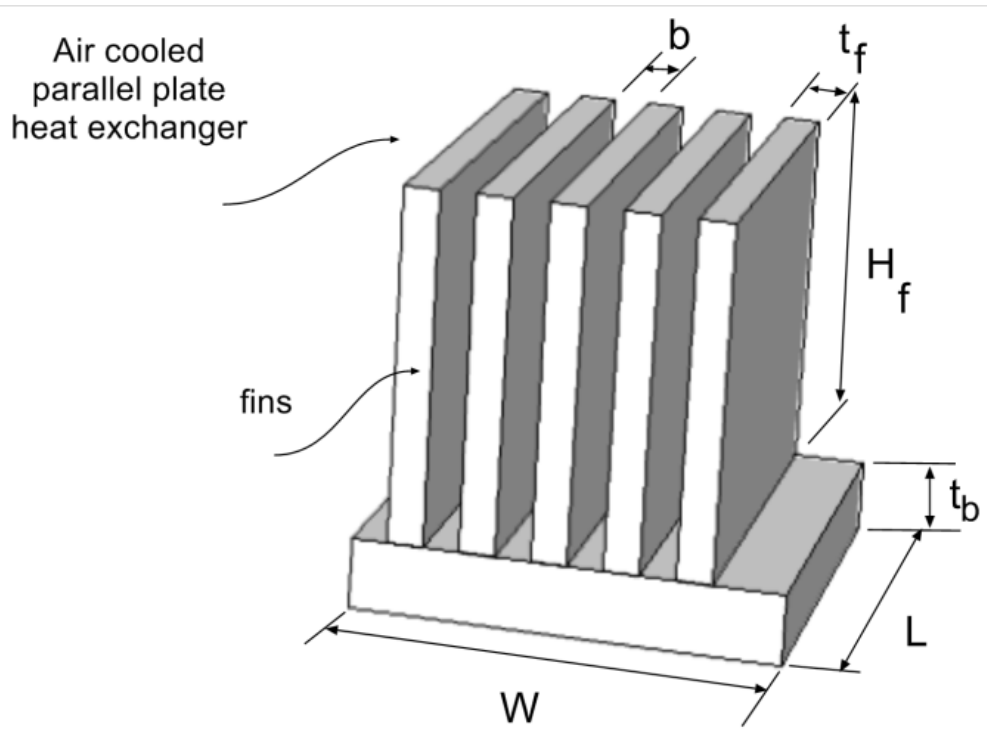

Figure 2-4 Parallel plate-fin heat exchanger 


\subsection{Results and Discussion}

To illustrate the application of the method described above, a TEG based selfcooling system is analyzed using certain defined parameters. For the sake of comparison, the heat engine working as internally and externally reversible Carnot engine and endoreversible heat engine are also simulated.

\subsubsection{Carnot heat engine}

The TEG based self-cooling system could be thought of as internally and externally reversible heat engine operating between heat source at $T_{d}$ and heat sink at $T_{f}$. Due to the assumption of reversibility, the hot side of TEG module is equal to the heat source temperature $\left(T_{h s}=T_{d}\right)$ and cold side temperature of TEG module is equal to heat sink temperature $\left(T_{c s}=T_{f}\right)$.

Figure 2-5 shows the variation of $\eta_{c}$ with the ratio of heat sink to heat source temperature. The heat sink temperature is fixed at $298 \mathrm{~K}$ and heat source temperature is varied from 298 to $596 \mathrm{~K}$. It is evident that as the temperature difference between the heat sink and source increases, the efficiency also increases. In addition, the heat input needed to produce a unit of work output $\left(Q_{H}^{\prime}\right)$ decreases as the efficiency of the Carnot heat engine increases. 


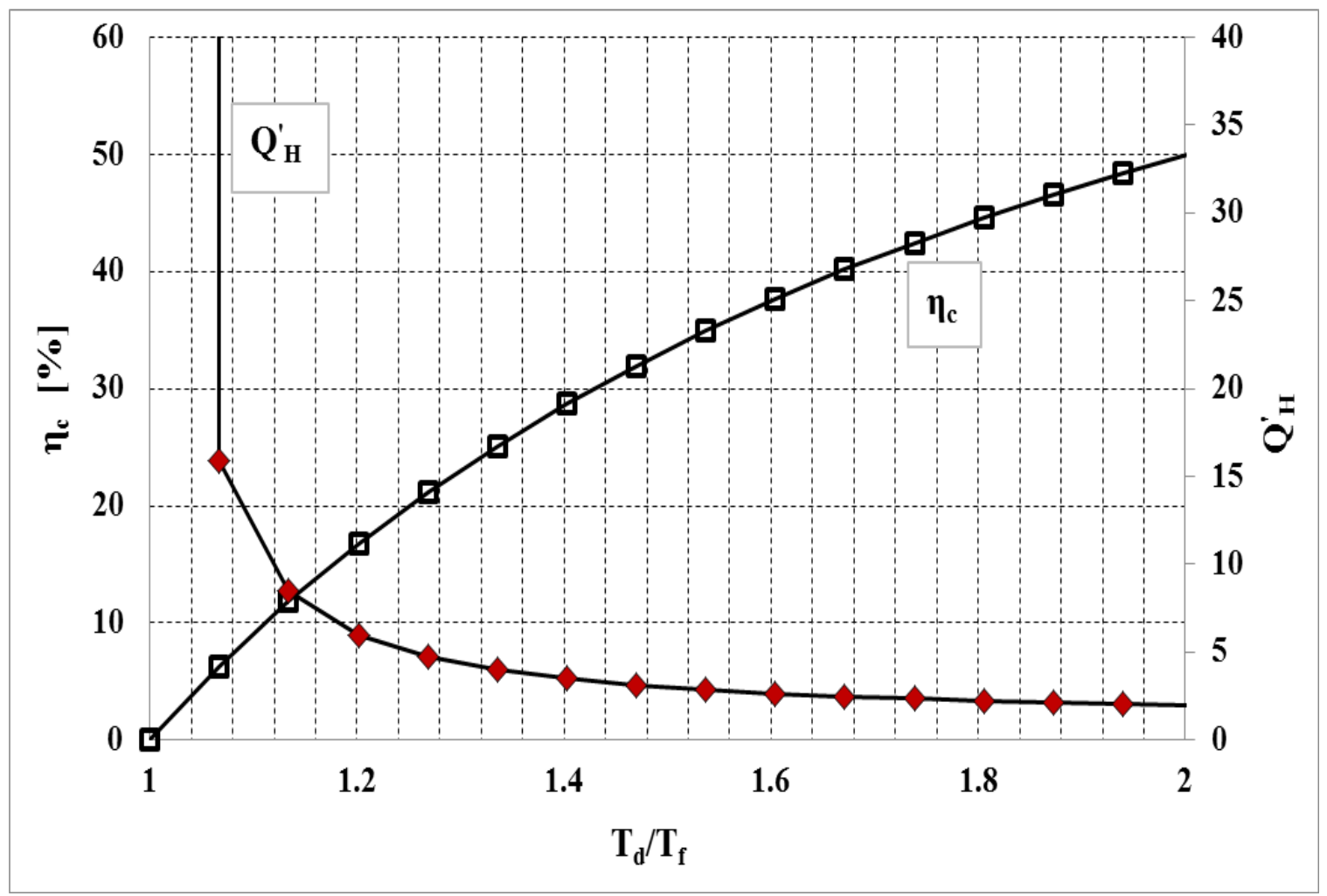

Figure 2-5 variation of $\eta_{c}$ with the ratio of heat sink to heat source temperature

However, as mentioned above, the analysis of Carnot heat engine is only useful to indicate the maximum efficiency and heat input required per unit of useful work for a system working between two temperatures. It needs an infinite amount of time and heat exchanger area to achieve the performance of Carnot heat engine. In addition, all internal and external irreversibilities are neglected.

\subsubsection{Endoreversible heat engine}

The performance of endoreversible heat engine is also studied to provide another bench mark for comparing the performance of real engines with idealized 
models. The heat sink temperature is fixed at $298 \mathrm{~K}$ and heat source temperature is varied from 298 to $596 \mathrm{~K}$.

The equations 2.1.6 - 2.1.9 are applied to investigate the efficiency $\eta_{c, \text { endo }}$ and heat input per unit of work produced by the heat engine $\left(Q_{H, \text { endo }}^{\prime}\right)$. The values of $(\mathrm{UA}) \mathrm{Hx}$ and $(\mathrm{UA}) \mathrm{cx}$ are fixed at $2.78 \mathrm{~W} / \mathrm{K}$ and $36 \mathrm{~W} / \mathrm{K}$. The comparison shows that the consideration of external irreversibility has decreased the efficiencies by as much as $66 \%$ as the temperature ratio $T_{f} / T_{d}$ of 0.5 . In addition for the temperature ratio of 0.5 , Carnot heat engine predicted that $3.5 \mathrm{~W}$ of heat is needed per $1 \mathrm{~W}$ of work produced while endoreversible heat engine model suggests that for an engine working between $598 \mathrm{~K}$ and $298 \mathrm{~K}$, the maximum heat released would be $1398 \mathrm{~W}$ and maximum power that could be produced would be $410 \mathrm{~W}$.

To put the results in the context of TEG based self-cooling system, it could be compared with an equivalent endoreversible heat engine as shown in Figure 2-6. In a typical self-cooling operation of thermoelectric generator, we can for instance assume the highest temperature at the device to be around $373 \mathrm{~K}$. For the type of heat exchangers specified (i.e. using the specified overall heat transfer coefficient), it could be inferred that (the heat that could be dissipated at maximum power) is around $336.58 \mathrm{~W}$. The maximum power from TEG module is $37 \mathrm{~W}$. 


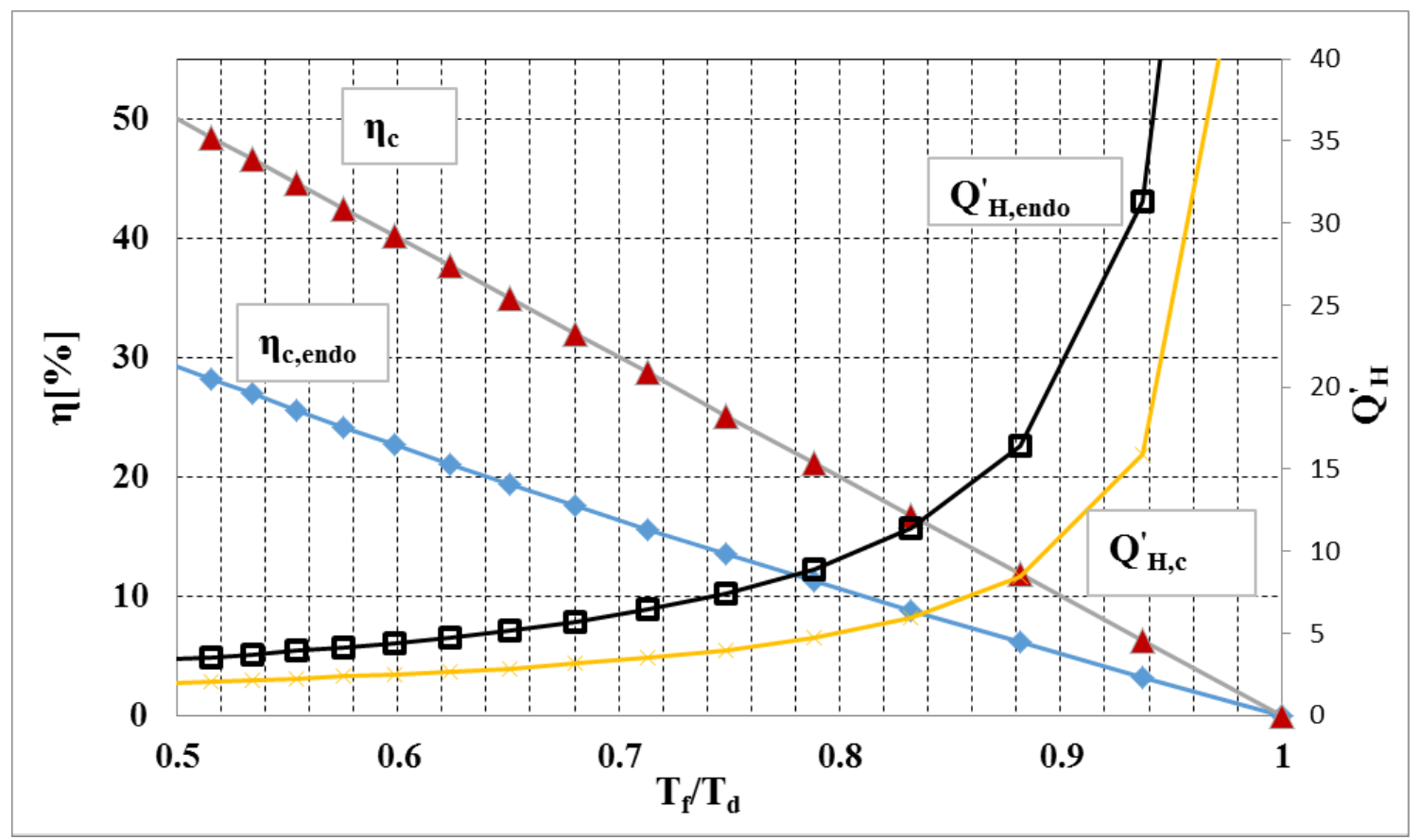

Figure 2-6 Comparison of Carnot heat engine and endoreversible heat engine

Endoreversible heat engine analysis does not contain any information about the type of process inside the main conversion engine. Moreover, it does not specify the size and internal conversion efficiency. It assumes internally reversible engine and the performance indicated is based on the interaction of the engine with the surrounding environment (reservoirs). Thus, it is obvious that the power from real self-cooling system would be much less than predicted by the endoreversible assumption. However, the endoreversible heat engine analysis provides useful information about the maximum possible power, heat input, and efficiency. It could serve as an important guide for comparison of real systems. 


\subsubsection{Externally and internally irreversible system}

The analysis of externally and internally reversible TEG based self-cooling system contains information about heat transfer which causes internal reversibility. These include Joule heating and conduction heat transfer. In addition, external irreversibility due to the interaction of TEG module with the hot and cold reservoirs has been represented. The following assumptions are also made.

a) Heat leakage from heat source (hot reservoir) to heat sink (cold reservoir) is not included. This assumption hold true for heat source with good insulation and/or high contact surface area of heat source with TEG module as compared to the sides. In these assumptions, the heat from heat source could be assumed to be completely transferred to the hot side of the TEG module.

b) Heat leakage from TEG module is not also considered. This is mostly a valid assumption considering that the thickness of TEG module is much less than (1/40) compared to the width or length of the modules.

c) Heat leakage from cold side heat sink to the cold reservoir is not included. The heat transfer is assumed to be from forced convection via the fin plates. 
d) The values of convection heat transfer coefficients and pressure losses are calculated using well documented analytical models. However, analytical models of convection heat transfer and pressure losses have errors and should be considered in context of providing a general guidance values rather than exact values of physical systems.

e) The analysis is based on Steady state assumption with isotopic and temperature independent properties.

The assumptions included above help in simplifying the model so that the major parameters are considered. The equations described in the previous sections are coded into Python and MATLAB programming scripts and the results are presented below. The code could be found in Appendix A

\subsubsection{Effect of varying size of cold side heat exchanger (UA) $c x$}

The system which depicts a TEG based self-cooling system has a heat source block with high conductivity. The contact size of heat source is fixed at $0.0016 \mathrm{~m}^{2}$ with overall heat transfer coefficient between $(U A)_{H X}$ between the heat source to the hot side of TEG module being $36 \mathrm{~W} / \mathrm{K}$. Therefore, the present system has a relatively low thermal resistance $(0.027 \mathrm{~K} / \mathrm{W})$ for heat transfer from heat source to the TEG module. 
For the cold side heat exchanger, a parallel plate-finned type heat dissipater with forced convection in air is considered. In this analysis, the size of the cold side heat exchanger is varied. A square profile is assumed for the heat exchanger. The base of the heat exchanger is varied from $0.0016 \mathrm{~m}^{2}$ to $0.0256 \mathrm{~m}^{2}$ corresponding to a side length of $40 \mathrm{~mm}$ to $160 \mathrm{~mm}$ respectively. Accordingly the value of $(U A)_{c x}$ ranges from $0.77 \mathrm{~W} / \mathrm{K}$ to $2.32 \mathrm{~W} / \mathrm{K}$. The cold side heat exchanger has a much higher thermal resistance than the hot side thermal resistance.

A TEG module of Kyrotherm TGM-287-1.0-1.5[64] is modeled and the values of parameters corresponding to the TEG model are considered. The smaller size of the heat exchanger corresponds to the size of TEG module $\left(0.0016 \mathrm{~m}^{2}\right)$. For the larger size of heat exchangers, a spreading resistance is considered.

As observed from Figure $2-7 a$ and $b$, for irreversible model both the hot side (Ths) and cold side (Tcs) temperatures of TEG module have decreased with an increase with $(U A) c x$. This is due to a decrease in thermal resistance in the cold side heat exchanger allowing more heat to be dissipated to the cold reservoir. For Ths, the decrease in temperature is only around $0.16 \%$ for a $66 \%$ increase in $(U A) c x$. This could be attributed to the high value of the ratio between $((U A) \mathrm{HX} /(U A) \mathrm{cx})$. The ratio ranges from 15.51 to 46 for $(\mathrm{UA}) \mathrm{Cx}$ value of 2.32 to $0.77 \mathrm{~W} / \mathrm{K}$ respectively. On the other hand, Tcs has decreased from close to 340 $\mathrm{K}$ to $318 \mathrm{~K}$. 
It is also evident from Figure 2-7a that the variation of $(\mathrm{UA})_{\mathrm{cx}}$ has also shown little effect on Ths for endoreversible model. As both endoreversible and irreversible models have same external irreversibilities on the hot side, they exhibit the same behavior in terms of variation of Ths. However, the effect of (UA)cx is markedly different between the endoreversible and irreversible models.

The endoreversible model has shown little sensitivity for a change in (UA)cx which may indicate its limits for evaluating the effect of cold side heat exchanger on $T_{\text {cs. }}$ From Figure 2-8, it is evident that endoreversible model has overestimated the power production from the heat engine and heat input for maximum power. TEG module heat engine is a direct heat to electricity heat conversion with finite heat transfer area. The endoreversible model does not make restriction on the area of the heat engine and thus indicate maximum positive heat transfer within the constraints of external irreversibility and the temperatures of the reservoirs. Thus, when the heat transfer area is made finite, the maximum heat transfer is also limited. At $(\mathrm{UA}) \mathrm{cx}$ of $2.32 \mathrm{~W} / \mathrm{K}$, only $1 / 6$ of the possible heat input is used in the reversible heat engine.

It is also possible to see from Figure 2-9 that irreversible model has a low efficiency $(\eta)$ reaching a maximum of $2.5 \%$ for the set of parameters tested. This is due to the inherent nature of TEG module which is direct heat to electricity conversion engines which has low conversion efficiency. The values depend on 
the Seebeck coefficients which basically relate temperature difference with voltage.

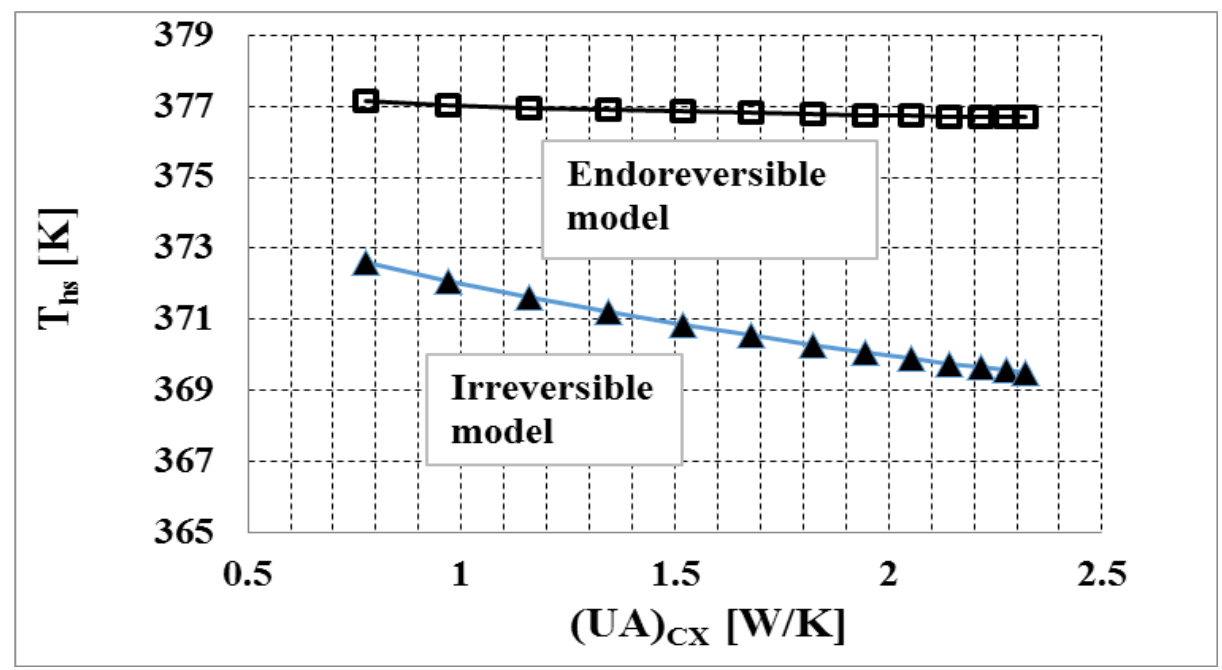

a)

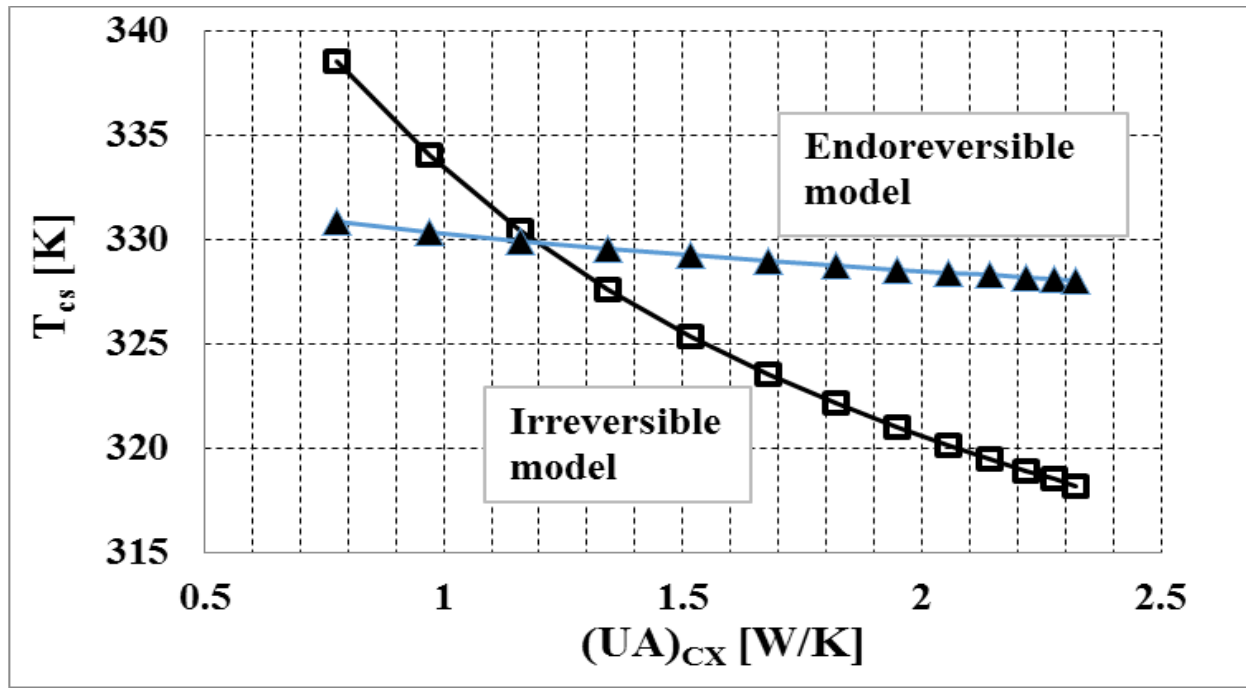

b)

Figure 2-7 Variation of a)Ths b) Tcs with (UA)cx 


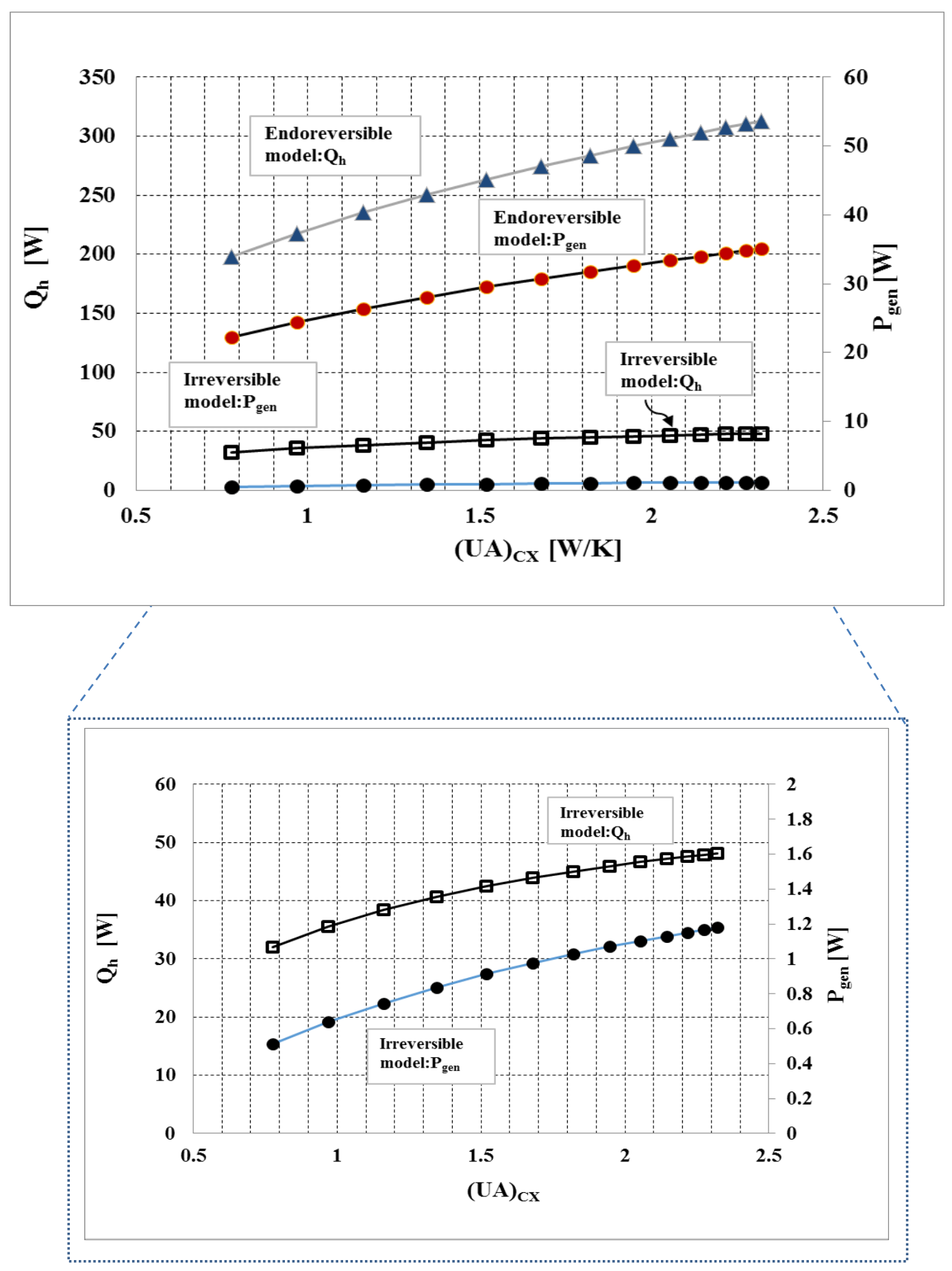

Figure 2-8 Variation of $Q_{h}$ and $P_{g e n}$ with $(U A) c x$ 


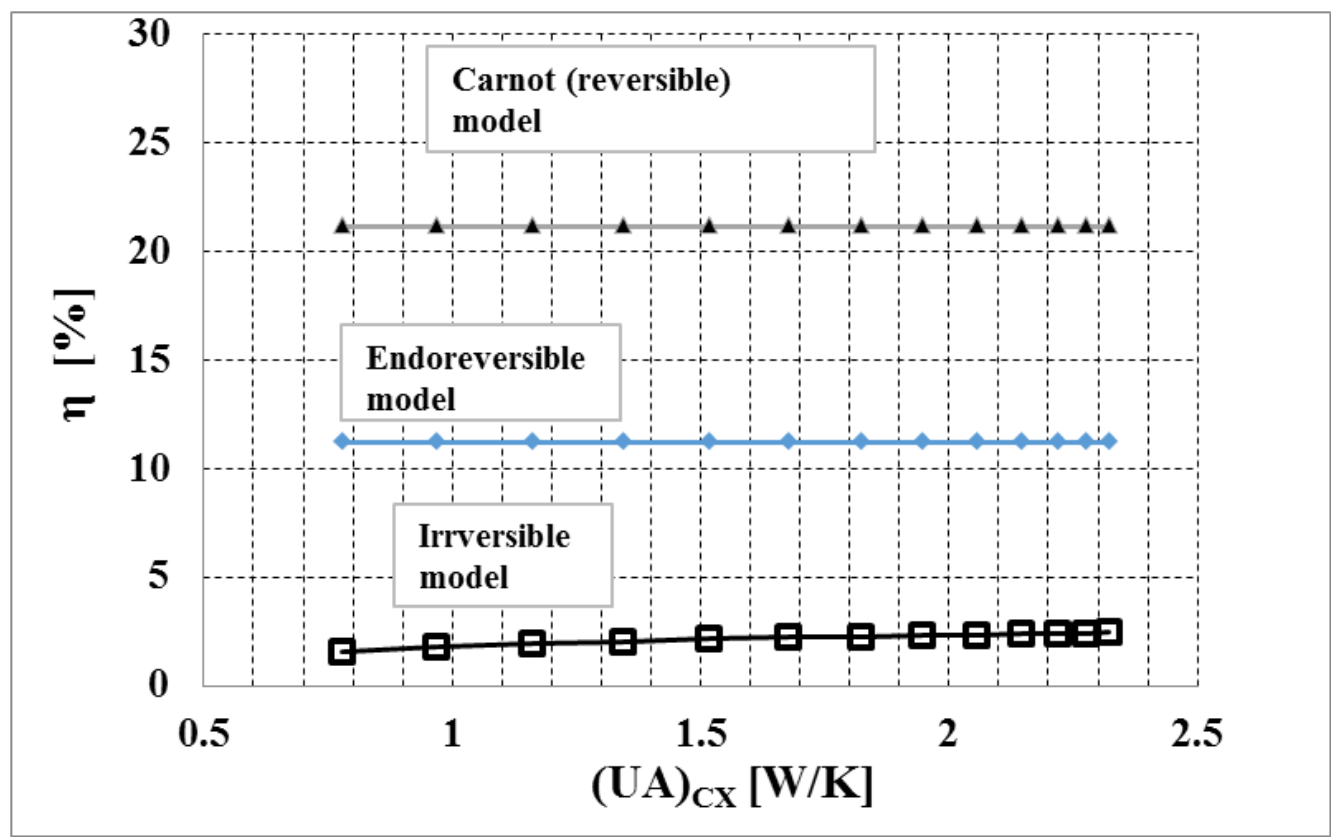

Figure 2-9 Variation of $\eta$ and $\Delta T_{h c}$ with $(U A) c x$

Presently, for the commercial solid material based TEG modules, the efficiency remains low. The endoreversible model and Carnot (internally and externally reversible) models have efficiency values of $11 \%$ and $21 \%$ respectively. The efficiency values in those models apply for any type of heat engine working under the parameters specified. One observes that the efficiency values are not affected much by the variation of $(\mathrm{UA}) \mathrm{cx}$. This is because both heat input and power change at the similar rates. It is also possible to see that TEG module engine has a more room for improvement to achieve performance that closely resembles the endoreversible or Carnot engines. 


\subsubsection{Effect of size of heat engine}

The effect of the size of heat engine could be investigated by replacing the heat engine by arrays of heat engines connected in series. This is equivalent to using array of TEG module connected in series electrically and in parallel thermally.

Equations 2.4.5 to 2.4.8 are again modified to account for the presence of multiple TEG modules.

$$
\begin{gathered}
Q_{h}=Q_{H X}=N_{m} I T_{h s} \alpha_{m}+\frac{\left(T_{h s}-T_{c s}\right)}{\Theta_{T E G, a r r a y}}-\frac{N_{m} R_{T E G}}{2} I^{2}=U_{H X} A_{H X}\left(T_{d}-T_{h s}\right) \quad 2.6 .1 \\
T_{h s}=\frac{U_{H X} A_{H X} T_{d}-\frac{N_{m}\left(T_{h s}-T_{c s}\right)}{\Theta_{T E G}}+\frac{N_{m} R_{T E G}}{2} I^{2}}{U_{H X} A_{H X}+N_{m} \alpha_{m} I} \quad 2.6 .2 \\
Q_{C}=Q_{C X}=N_{m} I T_{c s} \alpha_{m}+\frac{N_{m}\left(T_{h s}-T_{c s}\right)}{\Theta_{T E G}}+\frac{N_{m} R_{T E G}}{2} I^{2}=U_{C X} A_{C X}\left(T_{c s}-T_{f}\right) \quad 2.6 .3 \\
T_{c s}=\frac{U_{C X} A_{C X} T_{f}+\frac{N_{m}\left(T_{h s}-T_{c s}\right)}{\Theta_{T E G}}+\frac{N_{m} R_{T E G}}{2} I^{2}}{U_{C X} A_{C X}-N_{m} \alpha_{m} I}
\end{gathered}
$$

Where $\mathrm{N}_{\mathrm{m}}$ is the number of TEG modules connected in series electrically and in parallel thermally. 
Rewriting the equations on a TEG array basis:

$$
\begin{aligned}
& \left((U A)_{H X}+N_{m} K_{\text {TEG }}\right) T_{h s}+N_{m} \alpha_{m} I T_{h s}-(U A)_{H X} T_{d}-N_{m} K_{\text {TEG }} T_{c s}-0.5 N_{m} R_{T E G} I^{2}=0 \quad 2.6 .5 \\
& \left((U A)_{C X}+N_{m} K_{T E G}\right) T_{c s}-N_{m} \alpha_{m} I T_{c s}-(U A)_{C X} T_{f}-N_{m} K_{\text {TEG }} T_{h s}-0.5 N_{m} R_{T E G} I^{2}=0 \quad 2.6 .6 \\
& I=\frac{N_{m} \alpha_{m}\left(T_{h s}-T_{c s}\right)}{N_{m} R_{T E G}+R_{L}}
\end{aligned}
$$

The results from the analysis based on TEG array connected in series is shown in Figure 2-11. It can be observed from Figure 2-11 that increasing the area of TEG modules has resulted in an increase in open source voltage $\left(V_{o c}\right)$. Increasing the number of modules and hence the area of power conversion unit by $400 \%$ has resulted in more than $145 \%$ increase in voltage. This is due to an increase in the number of thermoelectric couples. However, the value of $V_{o c}$ has not increased by $400 \%$ in line with the increase in the number of thermoelectric couples. 


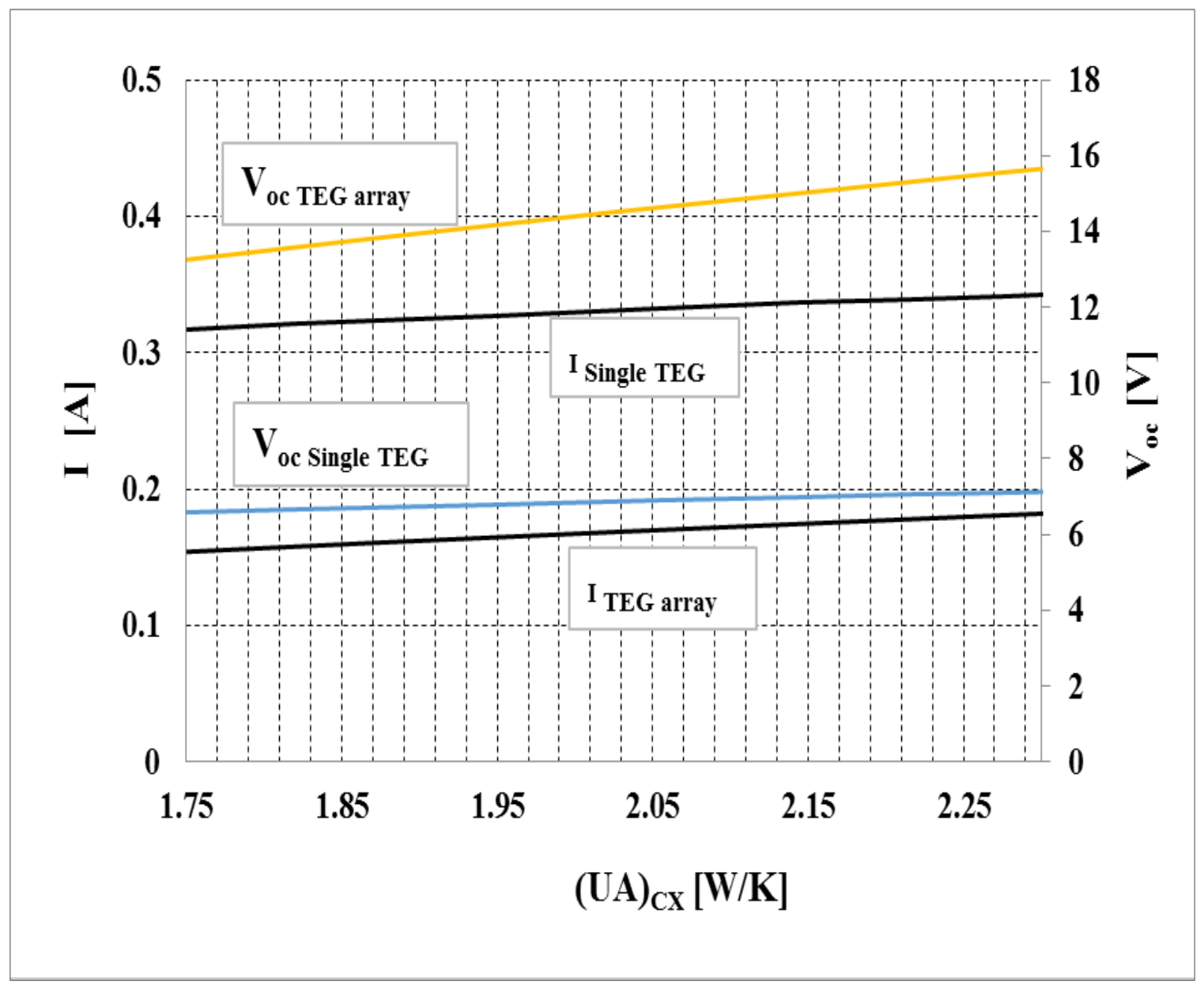

Figure 2-10 Comparison of $\mathrm{V}_{\mathrm{oc}}$ and I for single TEG module and array of TEG modules $\left(\mathrm{n}_{\mathrm{m}}=4\right)$

The current from TEG module array, on the other hand, has been halved as compared to single module. To investigate the reasons, it would be more helpful to look at the heat transfer in an array of modules as compared to a single module. 


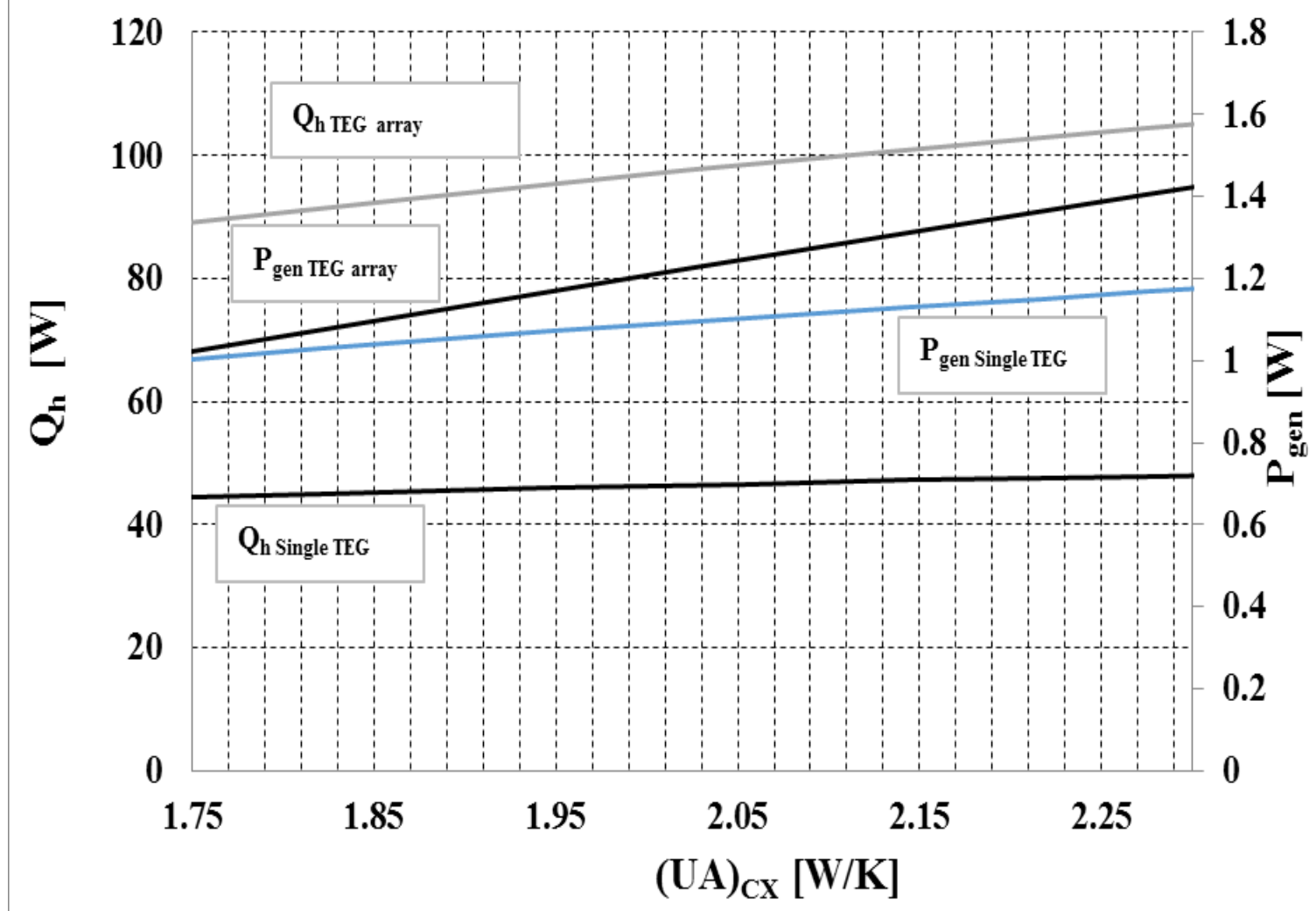

Figure 2-11 Comparison of $\mathrm{Q}_{\mathrm{h}}$ and $\mathrm{P}_{\mathrm{gen}}$ for single TEG module and array of TEG modules

The heat input to the TEG module increases by $250 \%$ for the TEG array. This could be attributed to the fact that as the area of TEG module reducing the thermal resistance to the heat flow.

However, the power rises by only $25 \%$ for (UA)cx equal to $2.6 \mathrm{~W} / \mathrm{K}$ for the TEG array as compared to single TEG module. 


$$
P_{\text {gen,max_array }}=\frac{N_{m}^{2} \alpha_{m}^{2}\left(T_{h s}-T_{c s}\right)^{2}}{4 R_{T E G, \text { array }}}
$$

If a TEG array could maintain the same temperature difference with a single module $\Delta T_{h s, \text { single }}=\Delta T_{\text {hs, array, }}$ then the equation reduces to:

$$
\begin{gathered}
P_{\text {gen,max_array }}=\frac{N_{m}^{2} \alpha_{m}^{2}\left(T_{h s}-T_{c S}\right)^{2}}{4 R_{T E G} N_{m}} \\
P_{\text {gen,max_array }}=\frac{N_{m} \alpha_{m}^{2}\left(T_{h s}-T_{c S}\right)^{2}}{4 R_{T E G}}
\end{gathered}
$$

This indicates that we expect the power to be multiple of the number of TEG modules in the array. However, $\Delta \mathrm{Ths,array}$ is a function of heat input into the TEG modules:

$$
\Delta T_{h s, \text { array }}=\Theta_{T E G, \text { array }} Q_{\text {in,array }}
$$




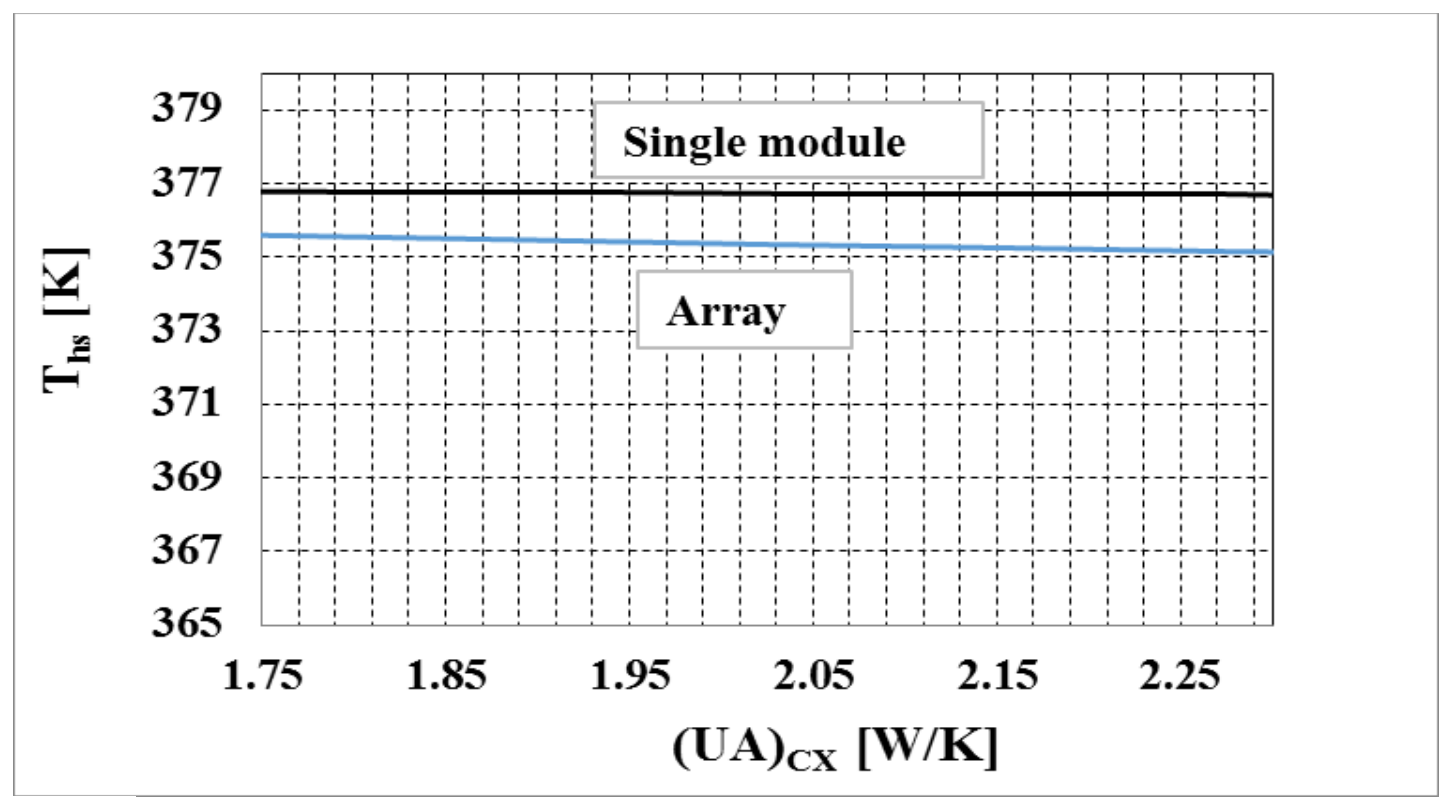

a)

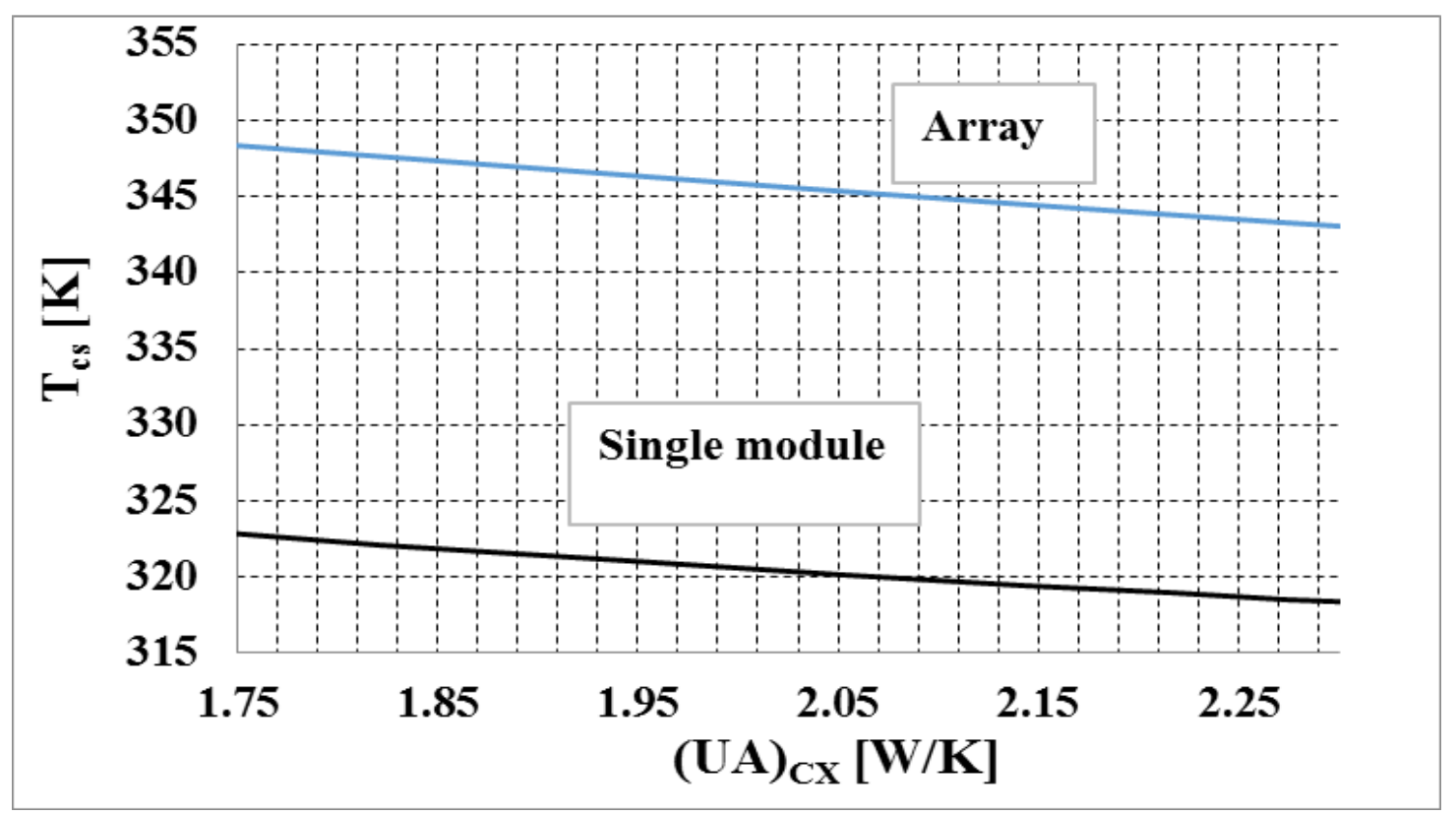

b)

Figure 2-12 Comparison of single TEG module and array of modules for a) Ths b) $T_{c s}$ 
As the TEG modules are connected in series and assuming the same thermal resistance for all the modules:

$$
\begin{gathered}
\Theta_{T E G, \text { array }}=\frac{\Theta_{T E G}}{N_{m}} \\
\Delta T_{h s, \text { array }}=\frac{\Theta_{T E G}}{N_{m}} Q_{i n, \text { array }}
\end{gathered}
$$

Thus, to maintain the same temperature difference, the heat input into the array of modules needs to be multiple of number of modules and heat input per module. If the heat input is less than that, then the temperature difference would also be less than the value for single module. The hot side temperature ( $T_{\text {hs }}$ ) of TEG modules is lower than a single array which allows for more heat to be transferred into the TEG module. (Figure 2-12). However, due to low thermal resistance as compared to single module, the cold side temperature of the array ( $\left.T_{c s}\right)$ is higher than single module.

\subsubsection{Self-cooling analysis}

The pressure loss and power consumption by the fan is integrated with the heat engine model to analyze the self-cooling potential of the whole system. For the previous analysis, the volume of air into the heat exchanger has been limited to $2.77 \times 10^{-3} \mathrm{~m}^{3} / \mathrm{s}$. In the present section for self-cooling, the volume of the air is 
varied from $0.34 \times 10^{-3} \mathrm{~m}^{3} / \mathrm{s}$ to $24.65 \times 10^{-3} \mathrm{~m}^{3} / \mathrm{s}$ or $1.22 \mathrm{~m}^{3} / \mathrm{h}$ to $88.7 \mathrm{~m}^{3} / \mathrm{h}$. The values correspond to $(\mathrm{UA}) \mathrm{cx}$ ranging from 0.44 to $6.28 \mathrm{~W} / \mathrm{K}$. It is to be noted that in self-cooling application, the volume of the fluid passing through the cold side heat exchanger is by itself a function of the power input into the fluid mover. Thus, it is the function of the power produced by TEG module arrays.

However, one could examine an independently varying volume flow rate to study the effect of volume flow rate of the fluid on different key parameters. The variation of $(U A) c x$ in the present analysis is only the function of the volume flow rate. The geometrical parameters of the cold side heat exchanger are kept constant at $\left(L=W=80 \mathrm{~mm}, \mathrm{H}_{\mathrm{f}}=10 \mathrm{~mm}, \mathrm{t} \_\mathrm{f}=1.5 \mathrm{~mm}, \mathrm{~N}_{-} \mathrm{f}=25, \mathrm{~b}=5 \mathrm{~mm}\right)$.

It can be observed from Figure 2-13 that with an increase in volume flow rate, the Reynolds number and pressure drop across the cold side heat exchanger increases exponentially. As the flow regime transforms from laminar to turbulent region, the flow would experience more pressure drop as it is commonly noted. However, it is interesting to see from Figure 2-14 that the hot side temperature of the TEG module does not change appreciably while the cold side has been cooled significantly. 


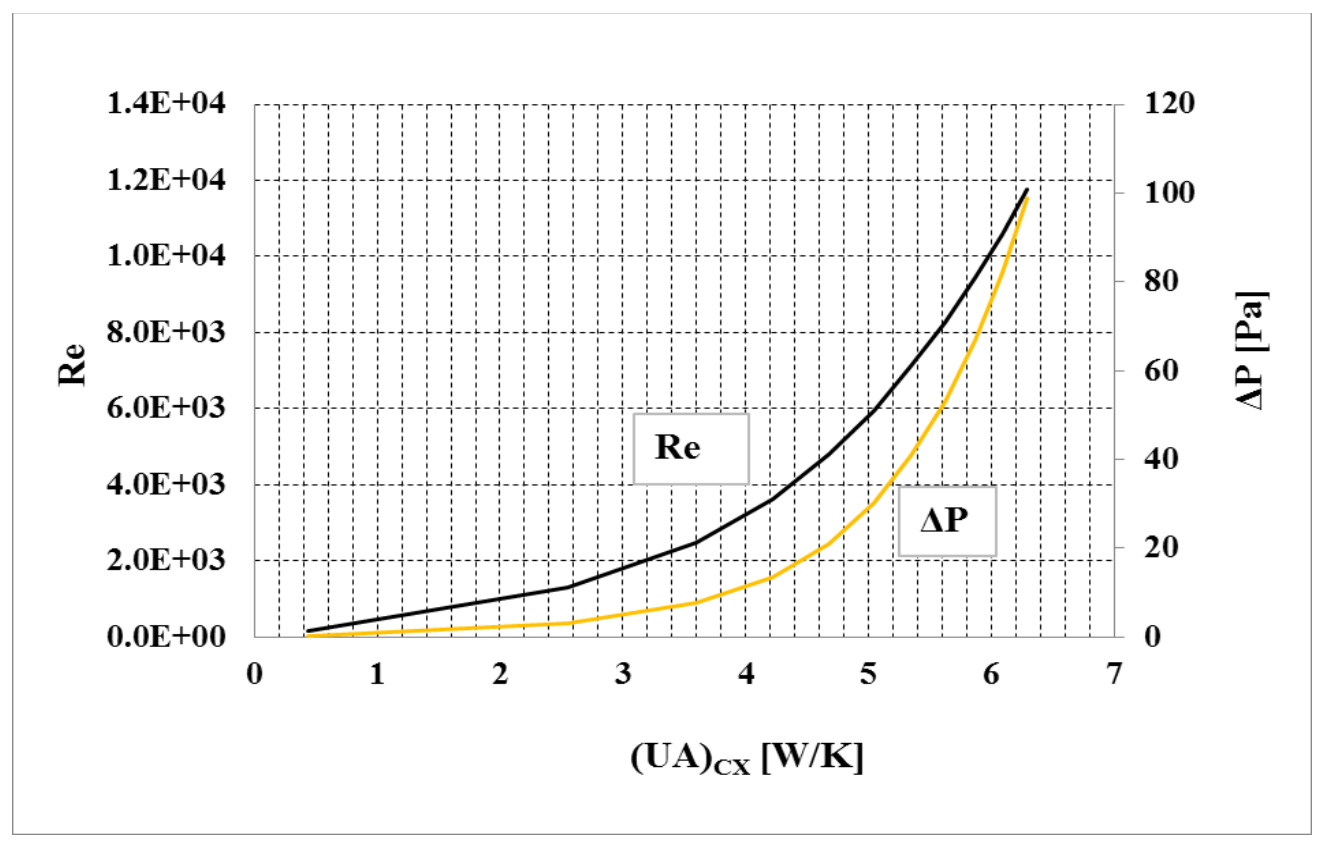

Figure 2-13 Variation of Reynolds number $(\mathrm{Re})$ and pressure drop $(\Delta \mathrm{P})$ with $(U A) c x$

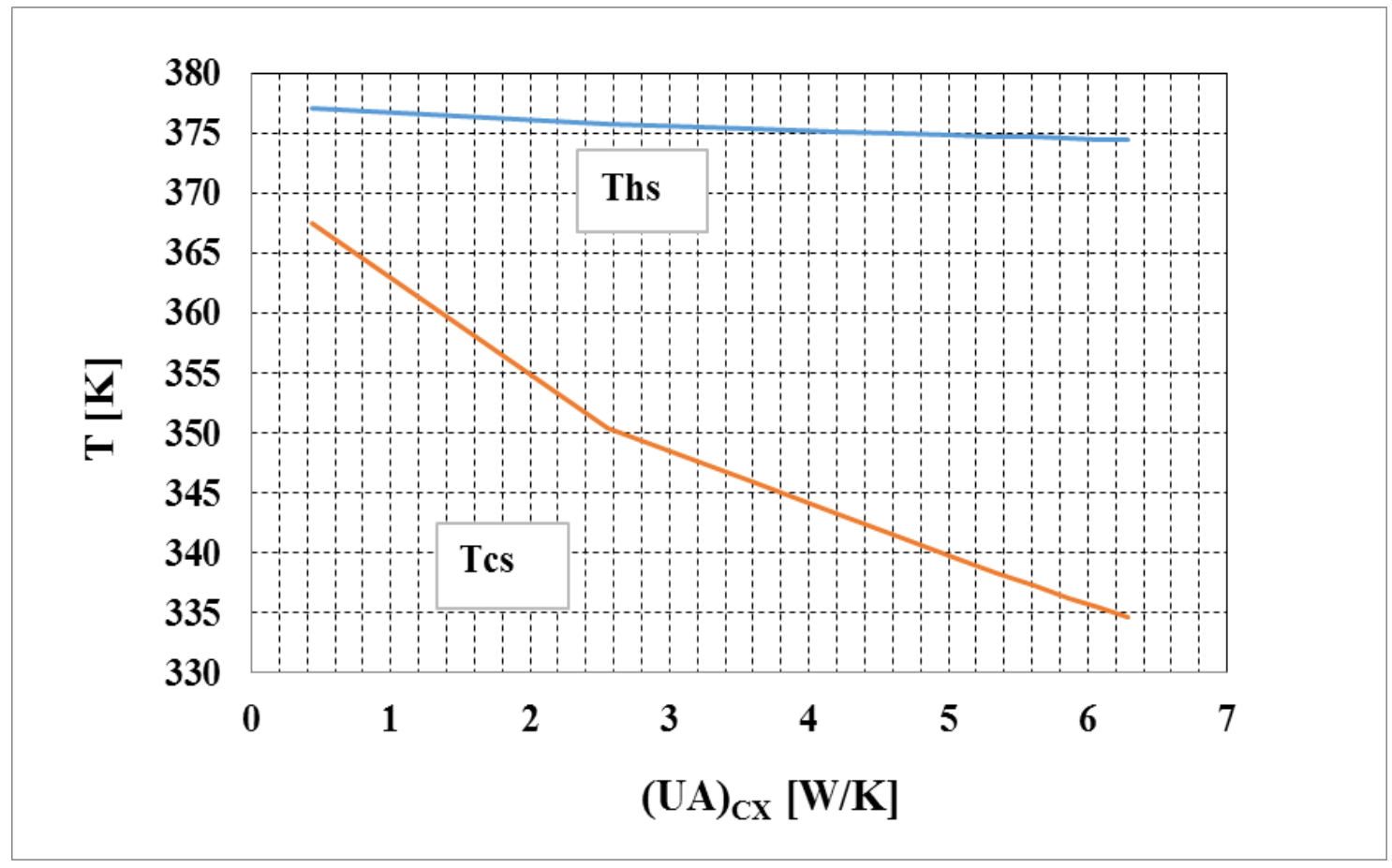

Figure 2-14 Variation of $T_{h s}$ and $T_{c s}$ with (UA)cx 
This is due to more flow and high velocity at the cold side heat exchanger resulting in high convection heat transfer. The temperature difference across the TEG modules also increased by close to $300 \%$ resulting in an equivalent percentage increases in $V_{o c}$ and I produced by TEG array (Figure 2-15). From power generation point of view, this could seem as a favorable aspect.

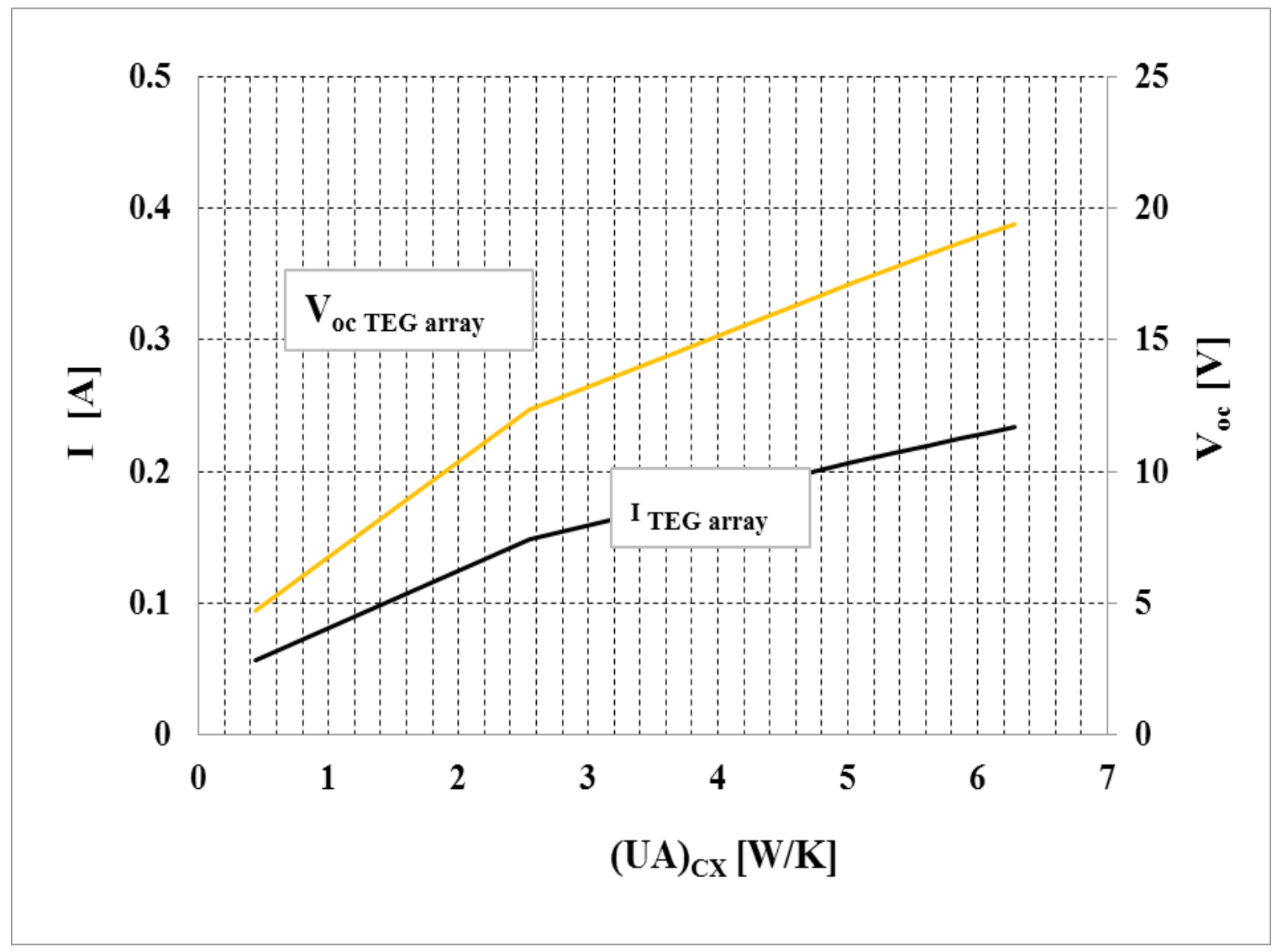

Figure 2-15 Variation of Voltage and Current produced by array of modules with $(\mathrm{UA})_{\mathrm{cx}}$

However, the fluid mover has to also consume power to move the fluid. Figure 2-16 shows that the power generated by TEG array $\left(P_{\text {gen }}\right)$ increases with $(U A) c x$ as it is expected due to more volume flow resulting in lower $T_{c s}$. But due to a rise 
in pressure drop in the cold side heat exchanger, the power consumed by the fluid mover also grows exponentially at high volume flow rate. As a result the net power from the system also drops and the system consumes more power when the volume flow rate reaches critical value. If the figure has been zoomed, it can easily be seen that the critical value of (UA)cx which resulted in zero net power is around $5.3 \mathrm{~W} / \mathrm{K}$ or Volume flow rate $(\mathrm{Vol})$ of $0.014 \mathrm{~m}^{3} / \mathrm{s}$ for the type of configuration used in this study.

From self-cooling point of view, higher power consumption does not necessarily result in lower device temperature as could be seen from the variation of $T$ hs. Thus it is imperative to stay within the range where net power is positive so that the system is able to run autonomously.

The model is very important to define the upper boundaries for parameters for fluid flow inside the cold side heat exchanger in terms of volume flow rate. But for the lowest boundaries of volume flow rates, it is important to look at factors like lowest voltage or power needed by a fluid mover such as fan to start. It is normally called a starting voltage or power. Thus, while it is tempting to look for the lowest possible power generation which could give net positive power, it should be at least higher than the lowest power or voltage requited to move the blades of the fluid mover. In addition, the fluid mover also has a unique performance curve and operates where the system curve intersects with fan 
performance curve which is also by itself a function of voltage input to the fluid mover.
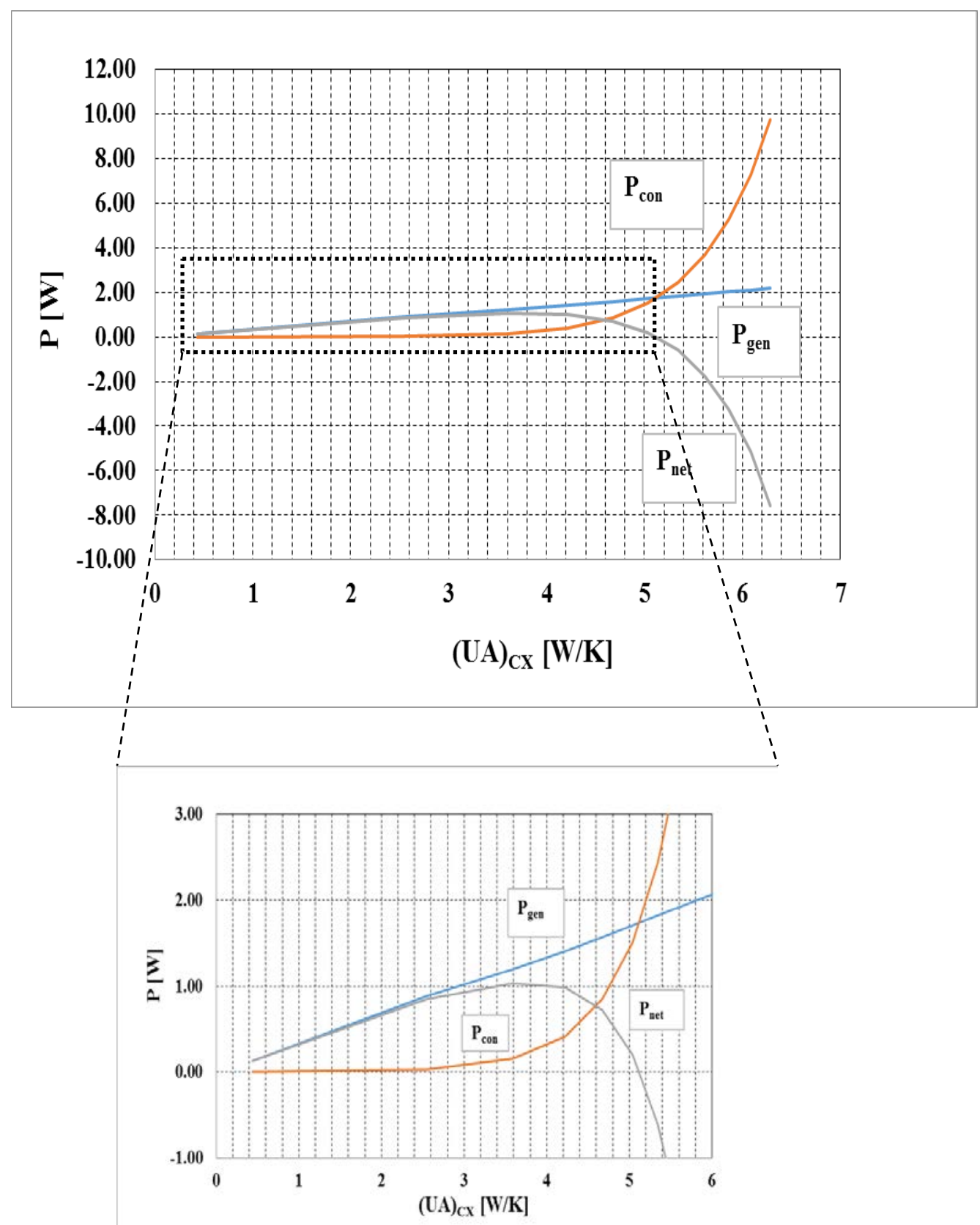

Figure 2-16 Variation of power generated by TEG arrays $\left(P_{g e n}\right)$, power consumed by fluid mover ( $P_{\text {con }}$ and net power in the system $\left(P_{\text {net }}\right)$ 
The fan performance curve is usually given at rated voltage. But it is also possible to construct the curves at various voltages. Once the range of volume flow rate which produces positive net power is identified, the fan-cold side heat sink system operation curve is superimposed on the fan performance curve. The combined curve shown in Figure 2-17 helps to identify system operation point. The system curve is extracted from manufactures data for SUNON Fan model EE92251S1-0000-A99[65].

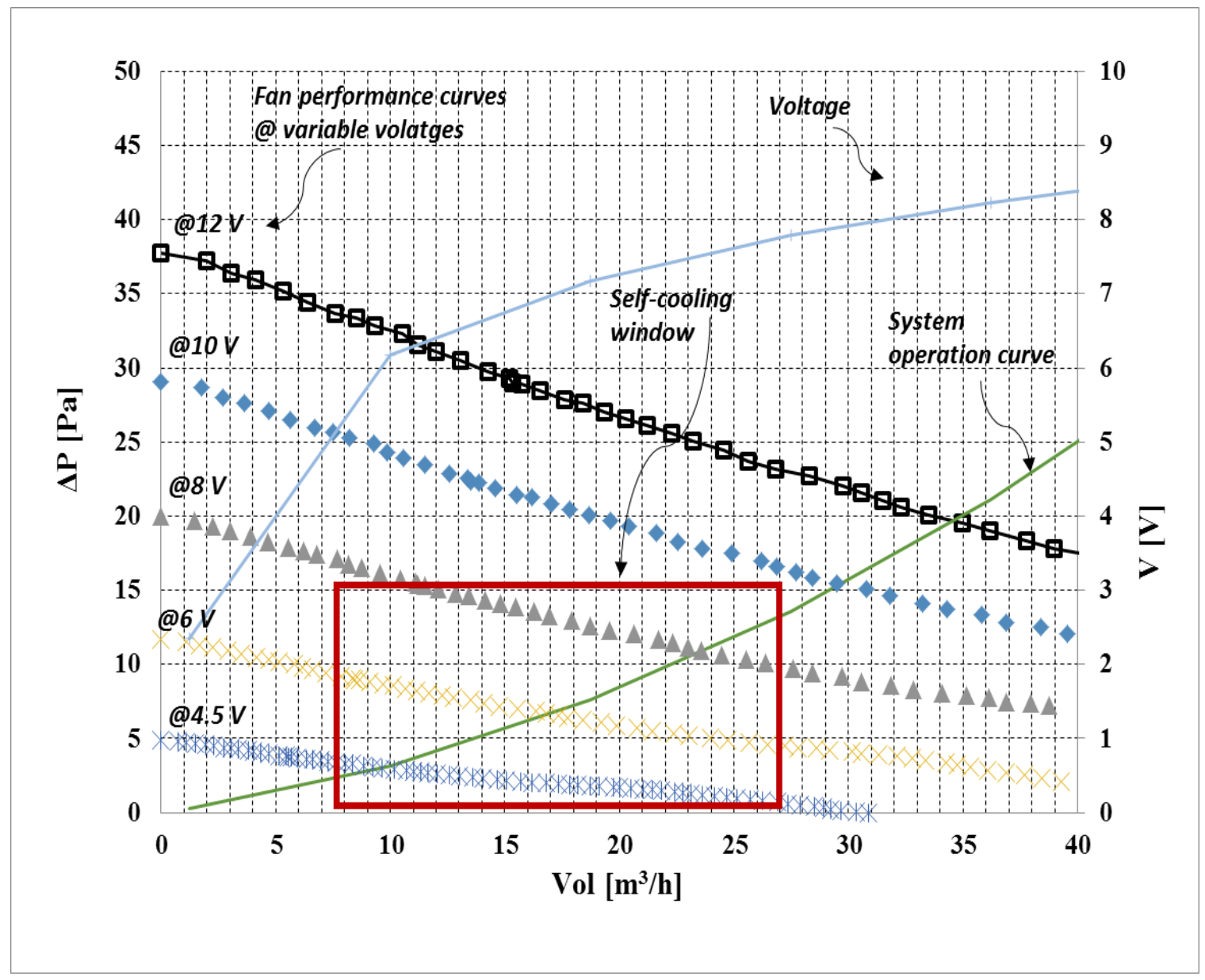

Figure 2-17 Self-cooling window 
It is evident from the figure that for volume flow rate more than $27 \mathrm{~m}^{3} / \mathrm{h}$, the system operation points lie below the operation point (for a particular input voltage).

However, a system operation points below $8 \mathrm{~V}$ are within the voltage provided by the TEG arrays. The lowest operation voltage (starting voltage) for the particular fan is $4.5 \mathrm{~V}$. Thus, the minimum volume flow rate is around $10 \mathrm{~m}^{3} / \mathrm{h}$.

Therefore we can define what is called "self-cooling window" which describes the range of volume flow rate possible for self-cooling for the particular geometrical configuration and type of fan used. Following the same procedures, the selfcooling window can be constructed for different geometrical configurations of the system and type of fluid mover (fan). 


\section{EXPERIMENTAL INVESTIGATION ON SELF-COOLING SYSTEM}

\subsection{Introduction}

There has only been very few experimental studies on TEG based self-cooling applications. Martinez et al. [37] performed an experimental demonstration of TEG driven self-cooling system. In their experiments, an array of TEG modules were placed directly on top of the heat source plate which enables the application of a more uniform and maximum temperature at the hot side of the TEG modules.

As the arrays are directly attached to the heat source, there is only a single thermal path. Therefore, the location of TEG modules in the primary heat path adds thermal resistance to the heat flow limiting the application of the system. In addition, in many practical applications a heat sink larger than the area of the heat source is utilized to provide more area for convection cooling. A heat spreader enables the distribution of the heat from a heat source to base of a larger heat sink area. Thus, in the present study a new arrangement of selfcooling in which an array of TEG module is placed between a heat spreader and cold side heat sink is investigated. Due to the position of TEG modules, the system has both primary and secondary heat paths. Moreover a new model is proposed where the TEG modules are placed only in the secondary heat paths reducing thermal resistance between the heat source and heat sink. 
The arrangement of the models is depicted in Figure $3-1$. The first case is a baseline model designated as case A. It consists of six TEG modules situated between the heat source device and heat sink. A heat spreader is used between the device and number of TEG modules arranged in rectangular array. The TEG modules have been labeled from TEG\# 1 to TEG\# 6. All the TEG modules are placed on top of a spreader.

For case A (Figure 3-1b), two TEG modules (TEG\# 3 and TEG\# 4) are placed centrally (primary heat path) while four TEG modules (TEG\# 1, TEG\# 2, TEG\# 5 and TEG\# 6) are placed on the sides (secondary heat path). TEG\# 1, TEG\# 3 and TEG\# 5 are on the front side with respect to the air flow from the fan while the rest are placed on the back side. For case B experiments (Figure 3-1c), TEG\# 3 and TEG\# 4 has been replaced by aluminum block of same size. The rest of the TEG modules have the same arrangement as case A.

This arrangement where all the TEG modules are placed on secondary heat path enables control of the temperature at a higher heat input by reducing thermal resistance. Case $\mathrm{C}$ represents a device set up where natural convection is used to cool the heat source without TEG modules. It functions as control model and is utilized to compare the performance of self-cooling in case A and case B. 


\subsection{Experimental setup}

An experimental set up (Figure 3-2) has been built to investigate the performance of self-cooling applications in case A, B and C. The heat source device is represented in the experiment by an aluminum plate with cartridge heater. The plate has a dimension of $80 \times 40 \times 20 \mathrm{~mm}^{3}$. A hole has been drilled in the aluminum plate equivalent to the dimensions of the cartridge heater of model Omega CSH-303535 with $12.7 \mathrm{~mm}$ diameter and around $76 \mathrm{~mm}$ in length. The heater has been tightly fitted inside the plate providing a uniform volumetric heating to the plate and has a maximum heating capacity and sheath temperature of $535 \mathrm{~W}\left(21 \mathrm{~W} / \mathrm{cm}^{2}\right)$ and $760{ }^{\circ} \mathrm{C}$ respectively

Thermal insulation material is wrapped around all exposed surfaces of the heated plate to prevent heat loss to the environment. An adjustable DC power source of MASTECH Model with variable DC output voltage from $0 \mathrm{~V}$ to $250 \mathrm{~V}$ and current up to $5 \mathrm{~A}$ is used to provide heating power to the heater. A digital meter of Model GPM-8212 from GW Instek provides a digital display of Voltage/Current/Wattage for precise control of the heating power input into the heating element.

An aluminum heat spreader of $3.8 \mathrm{~mm}$ thickness and an area of $120 \times 80 \mathrm{~mm} 2$ is used to conduct heat from the device to the array of TEG modules. 
Thermoelectric modules are sandwiched between the spreader and cold side heat sink.
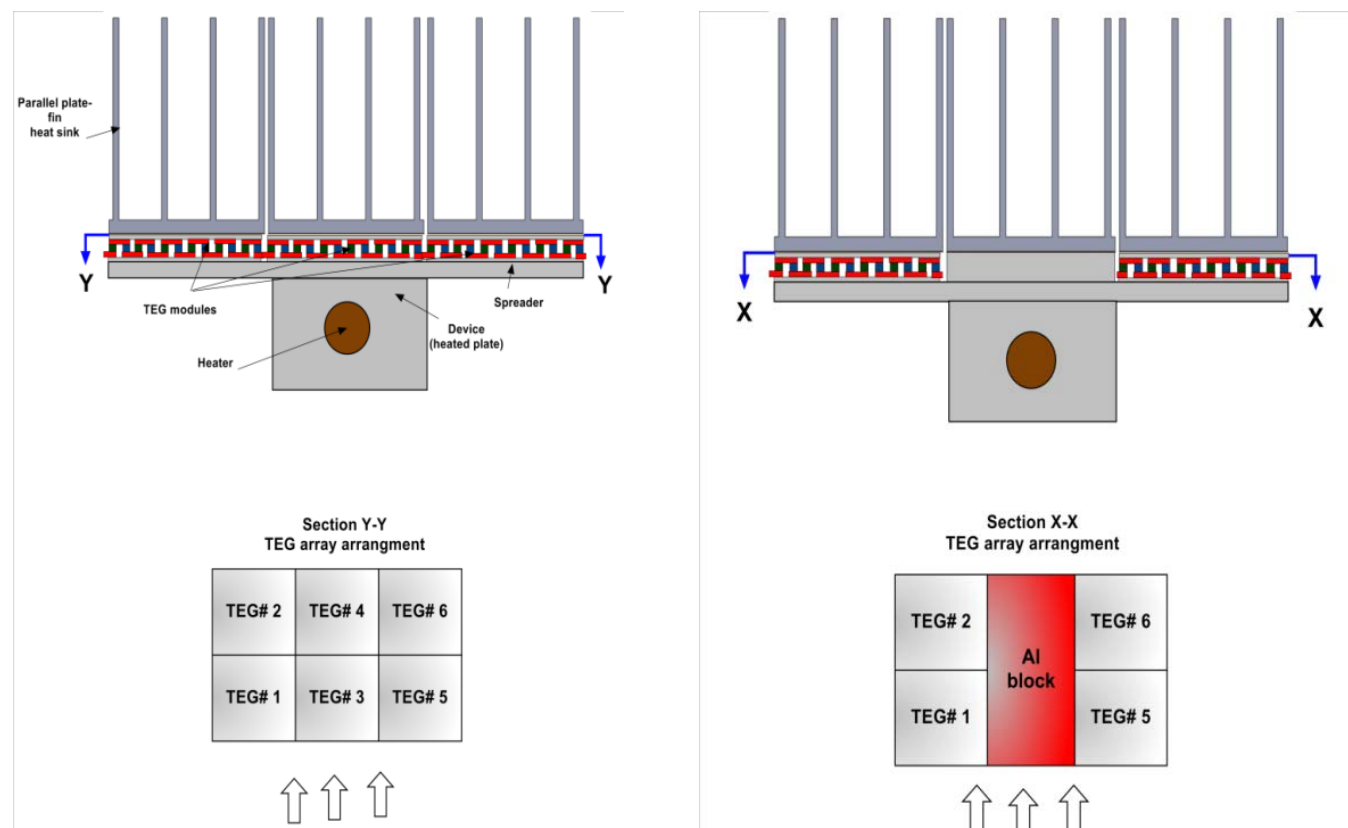

Air flow from fan

Case A

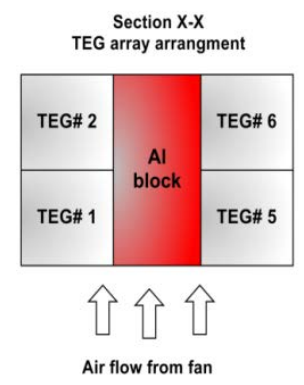

Case B

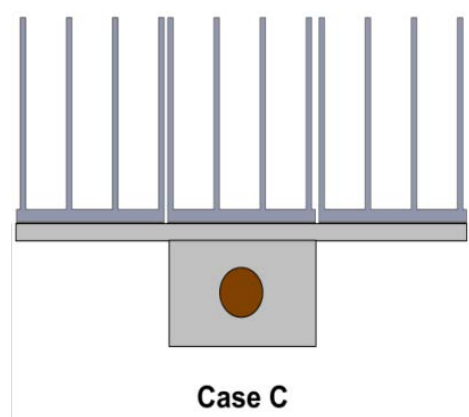

Figure 3-1 Geometrical configurations of cases A- 
An array of six TEG modules of EVERREDTRONICS TEG241-1.0-1.2 model has been used. Each module has an equal cold side and hot side area of $40 \times 40$ mm2 with 241 Bismuth Telluride thermoelectric couples inside each module. The detailed specification from the manufactures data is given in Table 3-1. The TEG modules have a maximum working temperature of $210^{\circ} \mathrm{C}$.

Three separate parallel plate fin heat sinks are placed on the cold side of the TEG modules. Each dissipater has a base area of $150 \times 40 \mathrm{~mm}^{2}$ and consists of 4 fins with fin height of $20 \mathrm{~mm}$ and a fin thickness of $1.5 \mathrm{~mm}$. The fin base is around $2 \mathrm{~mm}$ thick.

An OMEGATHERM high temperature and high thermal conductivity paste is used as an interface between all contacting surfaces to decrease contact resistance. A wind tunnel made of plexiglass and cross-sectional area $100 \times 120$ $\mathrm{mm}^{2}$ and $500 \mathrm{~mm}$ depth is used to direct air flow over the dissipater. A cooling fan of SUNON EE92251S1 model with dimensions of $92 \times 92 \times 25 \mathrm{~mm}$ is placed inside the wind tunnel at $40 \mathrm{~mm}$ upstream of the dissipater. The detailed specification for the fan is given in Table 3-2. 


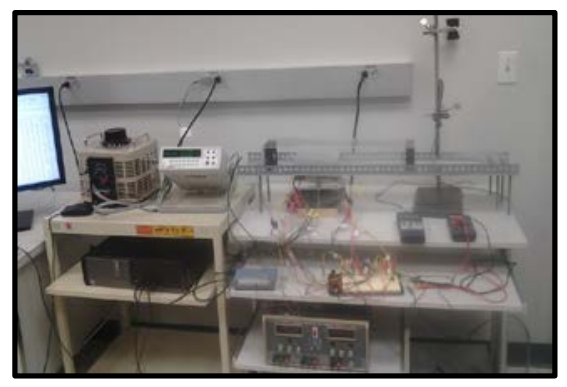

a)

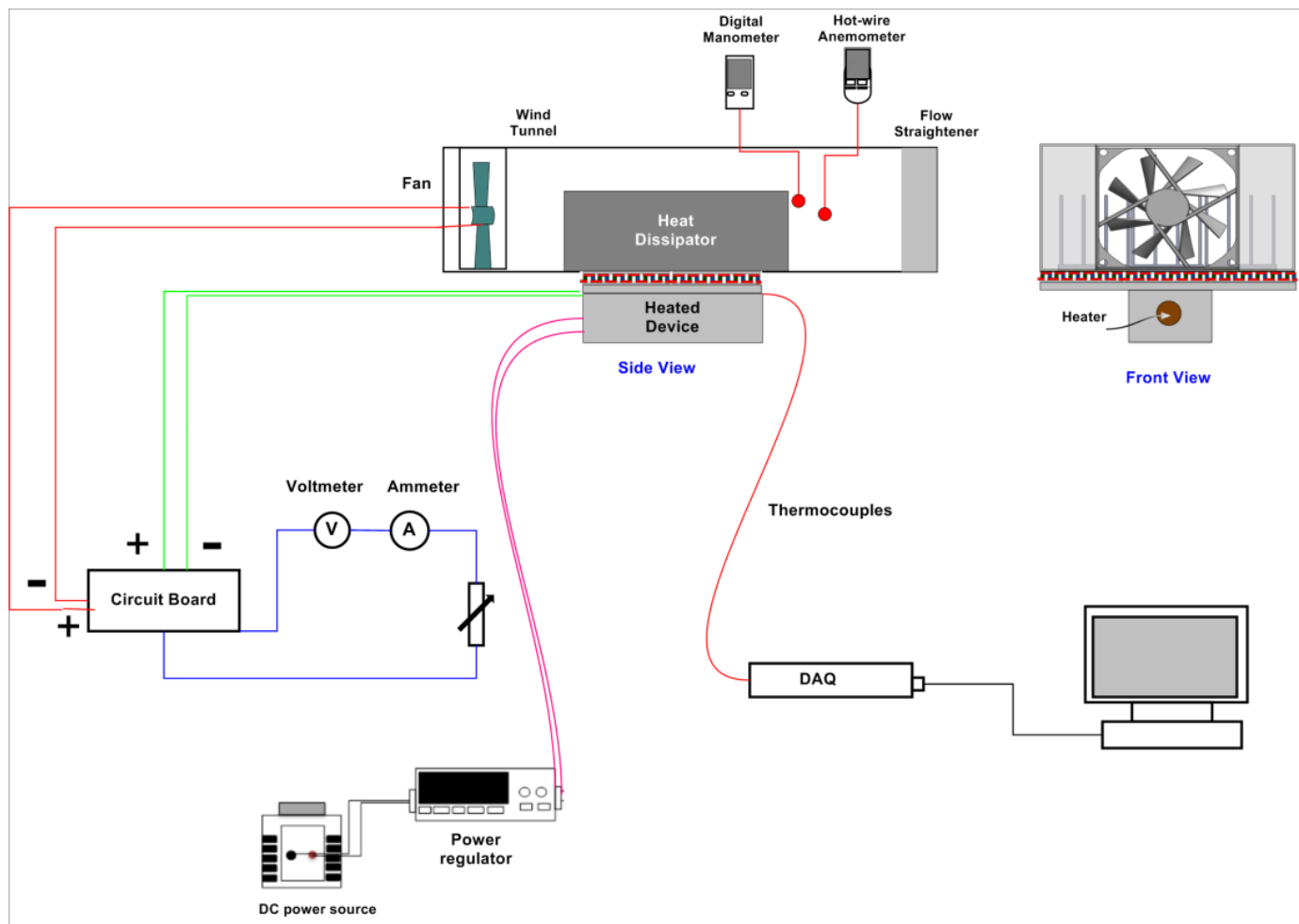

b)

Figure 3-2 a) picture of experimental setup and b) schematics of the investigation system 
Table 3-1 Specification of thermoelectric generator module ( model TEG241-1.01.2, China EVERREDTRONICS)

Dimensions $40 \mathrm{~mm} \times 40 \mathrm{~mm} \times 3.8 \mathrm{~mm}$

Based on hot side temperature of $160{ }^{\circ} \mathrm{C}$ and cold side of 50 ${ }^{\circ} \mathrm{C}$

\begin{tabular}{lllll}
\hline Open circuit & Matched & Matched & Electrical & Thermal \\
Voltage $\left(\mathrm{V}_{\text {oc }}\right)$ & Voltage & Power & resistance & Resistance \\
& & & & $\left({ }^{\circ} \mathrm{C} / \mathrm{W}\right)$ \\
$12.1 \mathrm{~V}$ & $6 \mathrm{~V}$ & $3.6 \mathrm{~W}$ & $10 \Omega$ & 1.5 \\
\hline
\end{tabular}

K-type glass-braided insulated thermocouples from OMEGA Engineering are used to measure temperatures at different sections of the experimental setup. A data acquisition module USB $1008 \mathrm{G}$ from MC Computing is utilized to log temperature data from the thermocouples and is connected to computer for data storage and analysis. 
An electrical circuit breadboard from JAMECO of JE25 model provides the platform for the TEG modules to be connected in a series arrangement electrically and at the same time allows for easier access for measurement of voltage and current coming from each module. A digital multimeter from WAVETEK (30XL model) is used to measure voltage and current from the TEG modules.

The air speed and temperature at the wind tunnel outlet is measured by a hot wire anemometer/thermometer (Control Company 4330 model). A digital manometer (OMEGA) measures the pressure drop in the heat sink. All tests were repeated three times to test the accuracy of the test data. The precision of the equipment and sample of uncertainty of the measured values are given in Table 3-3 and Table 3-4 respectively.

For all measured data, the mean value $(\bar{x})$ can be given as:

$$
\bar{x}=\frac{1}{m} \sum_{i=1}^{m} x_{i}
$$

The standard deviation $\left(S_{x}\right)$ and uncertainty of experimental data $(\sigma)$ could be expressed as[66]: 


$$
\begin{aligned}
& S_{x}=\left[\frac{1}{m-1} \sum_{i=1}^{m}\left(x_{i}-\bar{x}\right)^{2}\right]^{1 / 2} \\
& \sigma(\%)=\frac{S_{x}}{\bar{x}} \times 100
\end{aligned}
$$

For all measured data, the mean value $(\overline{\mathrm{x}})$ can be given as:

$$
\bar{x}=\frac{1}{m} \sum_{i=1}^{m} x_{i}
$$

The standard deviation $\left(\mathrm{S}_{\mathrm{x}}\right)$ and uncertainty of experimental data $(\sigma)$ could be expressed as[66]:

$$
\begin{aligned}
& S_{x}=\left[\frac{1}{m-1} \sum_{i=1}^{m}\left(x_{i}-\bar{x}\right)^{2}\right]^{1 / 2} \\
& \sigma(\%)=\frac{S_{x}}{\bar{x}} \times 100
\end{aligned}
$$

Table 3-2 Specification of DC brushless Fan ( model SUNON EE92251S1- 


\begin{tabular}{|c|c|c|c|c|c|}
\hline 0000-A & & & & & \\
\hline $\begin{array}{l}\text { Rated } \\
\text { Voltage }\end{array}$ & $\begin{array}{l}\text { Rated } \\
\text { Current }\end{array}$ & $\begin{array}{l}\text { Rated } \\
\text { Power }\end{array}$ & $\begin{array}{l}\text { Operating } \\
\text { Voltage } \\
\text { Range }\end{array}$ & Starting Voltage & $\begin{array}{l}\text { Electrical } \\
\text { Resistance }\end{array}$ \\
\hline $12 \mathrm{~V}$ & $165 \mathrm{~mA}$ & $2.0 \mathrm{~W}$ & $\begin{array}{l}4.5 \sim 13.8 \\
V\end{array}$ & $4.5 \mathrm{~V}$ & $72 \Omega$ \\
\hline
\end{tabular}

\begin{tabular}{|c|c|c|}
\hline Instrument & Parameter & Instrument precision \\
\hline \multirow{3}{*}{ GW Instek GPM-8213 Power meter } & Voltage & $\pm 0.1 \%$ of reading \\
\hline & Current & $\pm 0.1 \%$ of reading \\
\hline & W & $\pm 1 \%$ of reading \\
\hline \multirow{2}{*}{ Wavetek Digital multimeter(DC) } & Voltage & $\pm 0.15 \%$ of reading \\
\hline & Current & $\pm 0.5 \%$ of reading \\
\hline Omega K-type thermocouples & Temperature & $\pm 1 \%$ of reading \\
\hline \multirow[b]{2}{*}{ Control company Hotwire anemometer } & Velocity & $\pm 1 \%$ of reading \\
\hline & Temperature & $\pm 0.8 \%$ of reading \\
\hline
\end{tabular}




\begin{tabular}{|c|c|c|c|c|c|c|c|}
\hline $\begin{array}{l}\text { Temperature }\left({ }^{\circ} \mathrm{C}\right) \\
T_{d}\end{array}$ & & & & & & & \\
\hline$Q($ in) (W) & 50 & 60 & 70 & 80 & 90 & 100 & 110 \\
\hline Std.devav & 1.8 & 1.29 & 2.11 & 1.16 & 2.32 & 1.59 & 2.19 \\
\hline Uncertaintyav & $2.50 \%$ & $1.54 \%$ & $2.60 \%$ & $1.39 \%$ & $2.56 \%$ & $1.72 \%$ & $2.12 \%$ \\
\hline \multicolumn{8}{|l|}{$\begin{array}{l}\text { Voltage (V) } \\
\text { Case A }\end{array}$} \\
\hline Qin(W) & 70 & 70 & 70 & 70 & 70 & 70 & \\
\hline & TEG\# 1 & TEG\# 2 & TEG\# 3 & TEG\# 4 & TEG\# 5 & TEG\# 6 & \\
\hline Std.devav & 0.010 & 0.001 & 0.002 & 0.006 & 0.011 & 0.009 & \\
\hline Uncertaintyav & 1.77 & 0.10 & 0.32 & 0.87 & 1.00 & 1.32 & \\
\hline \multicolumn{8}{|l|}{$\begin{array}{l}\text { Current (A) } \\
\text { Case A }\end{array}$} \\
\hline$Q($ in) $(W)$ & 50 & 60 & 70 & 80 & 90 & 100 & 110 \\
\hline Std.devav & 3.055 & 1.527 & 2.516 & 3.055 & 4.163 & 3.055 & 2.081 \\
\hline Uncertaintyav & 5.72 & 2.29 & 3.05 & 3.29 & 4.02 & 2.71 & 1.71 \\
\hline
\end{tabular}

\subsection{Performance evaluation}

\subsubsection{Voltage and power}

The TEG modules are arranged in rectangular array with series electrical interconnections which enables the generation of larger values of voltage and hence power. Due to the position of TEG Modules, each experience an unequal amount of $\Delta T$ which results in different voltage output from each module. The total open circuit voltage $V_{\text {oc }}$ is expressed as: 


$$
V_{o c}=\sum_{i=1}^{n} V_{i}
$$

where $\mathrm{Vi}$ is the voltage from the $\mathrm{i}^{\text {th }}$ module in the series configuration and $\mathrm{n}$ is the number of TEG modules which is $n=6$ and 4 for case $A$ and $B$ configuration respectively.

The voltage flowing through the load, VL is given by:

$$
V_{L}=V_{o c}-V_{c c}
$$

where Vcc is the voltage at the array terminals and is measured by a digital multimeter.

The current flowing into the load I can be expressed as:

$$
I=\frac{V_{L}}{R_{L}}=\frac{V_{c c}}{n R_{T E G}}=\frac{V_{o c}}{n R_{T E G}+R_{L}}
$$

where RTEG and RL is the electrical resistance of a TEG module and load (fan) respectively. The manufactures reported value of RTEG and $R L$ are given in Table 3-1andTable 2 respectively. It is to be noted than the fan has a smaller electrical resistance of around $20 \Omega$ when stationary. 
The power supplied to the load (fan), PL is calculated by:

$$
P_{L}=V_{L} I_{L}
$$

The environment temperature, $\mathrm{Te}_{\mathrm{e}}$ is kept constant at $25^{\circ} \mathrm{C} \pm 0.3^{\circ} \mathrm{C}$ for all the experiment.

\subsubsection{Air flow resistance}

There are some important characteristics to consider when pressure drop for self-cooling applications are considered. The fan operating points are defined at the intersection points between the fan performance curves and system resistance curves. Fan manufactures usually test and provide performance curves at a rated voltage. However, the characteristic of self-cooling applications is that the voltage supplied to the fan is variable and could be different from rated voltage. It is, therefore, necessary to construct a pressure drop/ volume flow rate performance curve for the fan at different voltages. Moreover, in many applications of cooling devices using a fan, the devices are located inside an enclosure or compartment with inlet and outlet openings as they need to be shielded from the environment or outside interferences. Thus, it is vital to study total pressure drops inside an enclosure. 
For a constant impeller diameter and assuming constant fan efficiency in the frequency range, the following fan law [67] could be used to relate volume flow rate, $\dot{U}$, with the fan rotational speed, $\omega$, at different voltage input.

$$
\frac{\dot{U}_{x V}}{\dot{U}_{r V}}=\frac{\omega_{x V}}{\omega_{r V}}
$$

where $\dot{U} x V$ and $\dot{\omega} x V$ are the volume flow rate and fan rotational speed at a given voltage while ÜrV and $\dot{\omega} \mathrm{rV}$ are volume flow rate and fan rotational speed at the rated voltage. Another fan law equation which relates the pressure drop at any given voltage $(\triangle \mathrm{PxV})$ to the pressure drop at rated voltage $(\Delta \mathrm{PrV})$ is given by:

$$
\frac{\Delta P_{x V}}{\Delta P_{r V}}=\frac{\left(\omega_{x V}\right)^{2}}{\left(\omega_{r V}\right)^{2}}
$$

The ratio of fan supply power at a certain voltage $P_{x v}$ to the power at rated voltage $P_{r v}$ relates in cubic power to the fan rotational speed by:

$$
\frac{P_{x V}}{P_{r V}}=\frac{\left(\omega_{x V}\right)^{3}}{\left(\omega_{r V}\right)^{3}}
$$

The fan supply power is also related to the fan supply voltage $V x$ by the equation: 


$$
P_{x V}=\frac{\left(V_{x}\right)^{2}}{R_{L}}
$$

where $\mathrm{RL}$ is the internal electrical resistance of the fan

Combining the above two equations:

$$
\left(\omega_{x V}\right)^{3}=\frac{\left(V_{x}\right)^{2}}{R_{L} P_{r V}}\left(\omega_{r V}\right)^{3}
$$

Equations 3.3.5 and 3.3.6 could be written in terms of input voltage by substituting the relation from 3.3.8.

$$
\begin{aligned}
& \left(\frac{\dot{U}_{x V}}{\dot{U}_{r V}}\right)=\left(\frac{\left(V_{x}\right)^{2}}{R_{L} P_{r V}}\right)^{1 / 3} \\
& \left(\frac{\Delta P_{x V}}{\Delta P_{r V}}\right)=\left(\frac{\left(V_{x}\right)^{2}}{R_{L} P_{r V}}\right)^{2 / 3}
\end{aligned}
$$

\subsubsection{Thermal resistance}

The performance of self-cooling system could also be described in terms of thermal resistance for the heat flow inside the parts making up the whole 
system. A one dimensional thermal model is used to study the heat transfer process in the system by using network of thermal resistances as shown in Figure 3-3.

The description of the equations representing the thermal resistance in each part is given in Table 3-5. The heat from the device is represented by Qin. It passes from the device to the array of TEG modules via a heat spreader after crossing the conduction thermal resistance inside the device $\left(\Theta_{\mathrm{dc}}\right)$ and contact and spreading thermal resistance between device and spreader $\left(\Theta_{\mathrm{sp}}\right)$ and spreader conduction thermal resistance $\left(\Theta_{\mathrm{sc}}\right)$. After the heat enters the spreader, it could take three different paths before the heat is rejected to the ambient.

The first path is the primary heat path represented by $\mathrm{Q}_{h, 3}$ and $\mathrm{Qh}_{\mathrm{h}, 4}$ through thermal resistance of $\Theta_{T E G, 3}$ and $\Theta_{T E G, 4}$ respectively. In case $A, \Theta_{T E G, 3}$ and $\Theta_{T E G, 4}$ are the thermal resistance of TEG modules while in case B they are replaced by aluminum blocks. The second and third heat paths are secondary or shunt heat paths. These are $\mathrm{Q}_{h, 1}, \mathrm{Qh}_{\mathrm{h}, 2}, \mathrm{Q}_{\mathrm{h}, 5}$ and $\mathrm{Q}_{\mathrm{h}, 6}$. For both cases $\mathrm{A}$ and $\mathrm{B}, \Theta_{\mathrm{TEG}, 1}$, $\Theta_{\mathrm{TEG}, 2,}, \Theta_{\mathrm{TEG}, 5}$ and $\Theta_{\mathrm{TEG}, 6}$ are thermal resistances of TEG modules. Finally, the heat passes through the heat sinks before it is rejected to the ambient air at temperature of $\mathrm{Ta}$. There are three separate heat sinks for the primary heat path and two symmetric secondary heat paths. 
The thermal resistance could also be grouped into three major parts. The first part is the thermal resistance network from the device to the hot side of the array of TEG modules is:

$$
\Theta_{d-T E G}=\Theta_{d c}+\Theta_{s p}+\Theta_{s c}=\frac{\left(T_{d}-T_{h, a v}\right)}{Q_{i n}}
$$

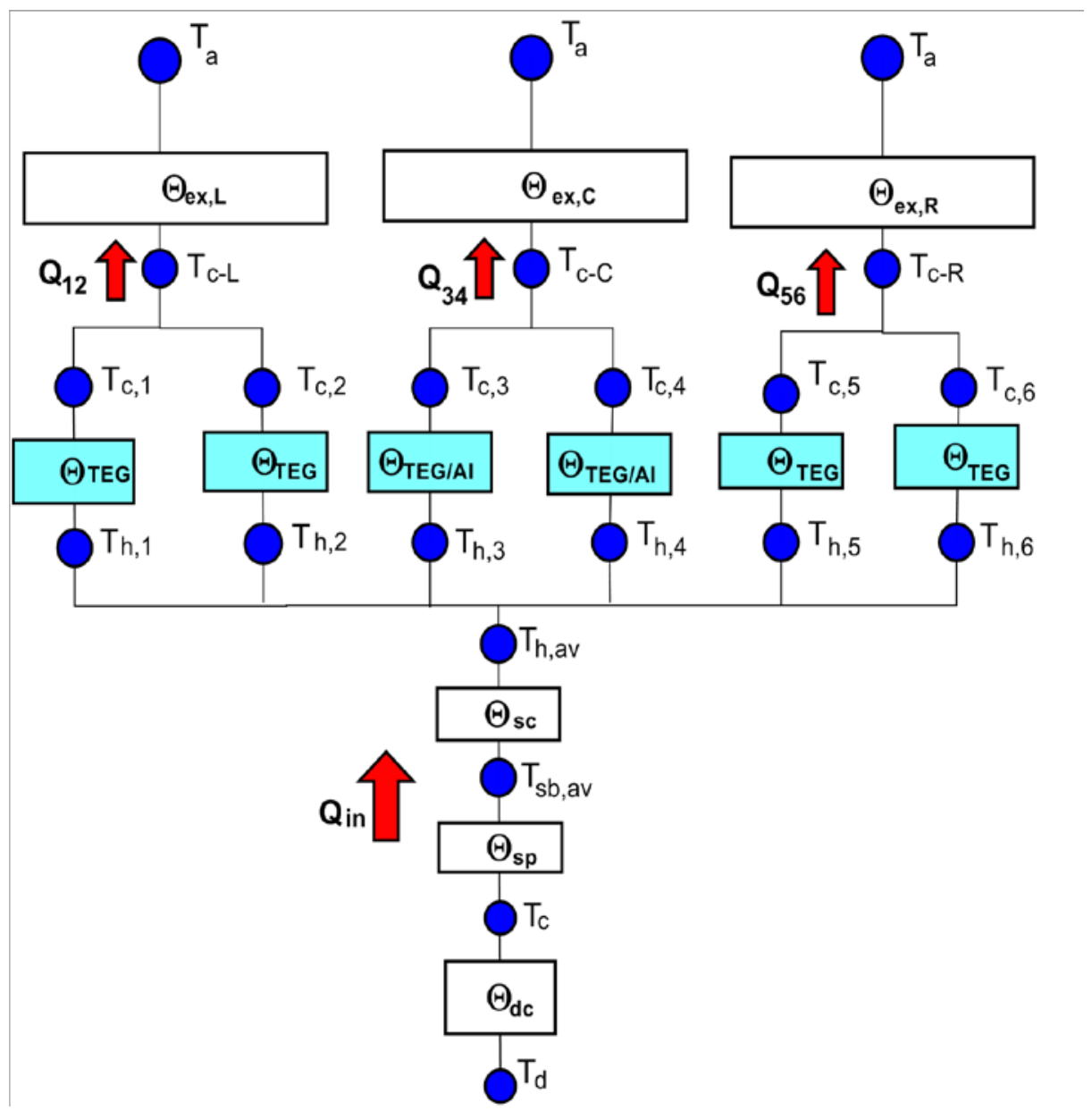

Figure 3-3 Thermal resistance diagram 


\section{Table 3-5. Description and expressions for thermal resistances}

\section{Symbol Description Equation}

$\Theta_{\mathrm{dc}} \quad$ Conduction: Inside the device $\quad \frac{T_{d}-T_{c}}{Q_{i n}}$

\begin{tabular}{lll}
\hline & Contact: $\mathrm{b} / \mathrm{n}$ device and heat \\
$\Theta_{\mathrm{sp}}$ & spreader + Spreading: $\mathrm{b} / \mathrm{n}$ device and $\frac{T_{c}-T_{\text {sb.av }}}{Q_{\text {in }}}$ \\
& heat spreader
\end{tabular}

$\Theta_{\mathrm{sc}} \quad$ Conduction: Inside spreader $\quad \frac{T_{s b, a v}-T_{h, a v}}{Q_{i n}} \quad \begin{aligned} & \mathrm{Th}, \mathrm{av}=\operatorname{avg}\left(\mathrm{T}_{\mathrm{h}, \mathrm{j}}\right) \\ & \mathrm{j}=1-6 \text { (case A) } \\ & \mathrm{j}=1-4 \text { (case B) }\end{aligned}$

\begin{tabular}{|c|c|c|c|}
\hline$\Theta_{\mathrm{TEG}, \mathrm{j}}$ & $\begin{array}{l}\text { Contact: } \mathrm{b} / \mathrm{n} \text { spreader and TEG } \\
\text { module + Conduction: Inside TEG } \\
\text { module+ Contact: } \mathrm{b} / \mathrm{n} \text { TEG module } \\
\text { and heat dissipaters }\end{array}$ & $\frac{T_{h, j}-T_{c, j}}{Q_{h, j}}$ & $\begin{array}{l}j=1-6(\text { case } A) \\
j=1-4(\text { case } B)\end{array}$ \\
\hline$\Theta_{e x, L}$ & $\begin{array}{l}\text { Conduction: Inside LSD }+ \\
\text { Convection: b/n LSD and surrounding } \\
\text { air }\end{array}$ & $\frac{T_{c-L}-T_{a}}{Q_{c, 12}}$ & $T_{C-L}=\operatorname{avg}\left(T_{c, 1}\right.$ and $\left.T_{c, 2}\right)$ \\
\hline$\Theta_{\mathrm{ex}, \mathrm{C}}$ & $\begin{array}{l}\text { Conduction: Inside CSD +Convection: } \\
\text { b/n CSD and surrounding air }\end{array}$ & $\frac{T_{c-C}-T_{a}}{Q_{c, 34}}$ & $T_{c-c=} \operatorname{avg}\left(T_{c, 3}\right.$ and $\left.T_{c, 4}\right)$ \\
\hline$\Theta_{\text {ex,R }}$ & $\begin{array}{l}\text { Conduction: Inside RSD + Convection } \\
\text { b/n RSD and surrounding air }\end{array}$ & $\frac{T_{c-R}-T_{a}}{Q_{c, 56}}$ & $T_{c-R}=\operatorname{avg}\left(T_{c, 5}\right.$ and $\left.T_{c, 6}\right)$ \\
\hline
\end{tabular}

LSD=Left-hand side heat dissipater; $C S D=$ Central heat dissipater; RSD=Right hand side heat dissipater 
The second part is thermal resistance inside the array of TEG modules, OTEGarray which could be described by the analysis of heat transfer in the TEG modules. The heat transfer inside the TEG modules at the hot side could be described as:

$$
Q_{h, j}=I T_{h, j} \alpha_{m}+\frac{\left(T_{h, j}-T_{c, j}\right)}{\Theta_{T E G, j}}-\frac{R_{T E G}}{2} I^{2}
$$

And at the cold side of the modules:

$$
Q_{c, j}=I T_{c, j} \alpha_{m}+\frac{\left(T_{h, j}-T_{c, j}\right)}{\Theta_{T E G, j}}+\frac{R_{T E G}}{2} I^{2}
$$

where $\alpha_{m}$ is the Seebeck coefficient for a TEG module. The first terms in Eq. 16 and $17 \mathrm{ITh,jam}$ and I $\mathrm{T}_{\mathrm{c}, \mathrm{jam}}$ are the Peltier heat terms at the hot side and cold side of TEG module respectively. Second term in both equations is the conduction heat terms and the last term represent Joule heating inside the modules. The difference between the heat transfer in hot side and cold side is the power generated from a TEG module which is given by the power generated due to Peltier effect minus the parasitic power loss in the conductor due to joule heating:

$$
Q_{h, j}-Q_{c, j}=I\left(T_{h, j}-T_{c, j}\right) \alpha_{m}-R_{T E G} I^{2}=P_{j}
$$


The TEG modules are connected in parallel thermally and the overall resistance of the TEG modules, $\Theta_{\text {TEG-array }}$ is given in case A Eq. 3.3.16 and case B Eq.3.3.17

$$
\begin{gathered}
\Theta_{\text {TEG-array }}=\left(\sum_{j=1}^{m}\left(\frac{1}{\Theta_{T E G, j}}\right)^{-1}\right)^{-1}=\left(\sum_{j=1}^{m}\left(\frac{1}{Q_{h_{j}}-I T_{h, j} \alpha_{m}+\frac{R_{\text {TEG }} I^{2}}{2}}\right)^{-1}\right)^{-1}=\frac{T_{h, a v}-T_{c, a v}}{Q_{i n}-\sum_{j=1}^{m}\left(I T_{h, j} \alpha_{m}+\frac{R_{T E G} I^{2}}{2}\right)} \\
\Theta_{\text {TEG-array }}=\left(\sum_{j=1}^{m}\left(\frac{1}{\Theta_{\text {TEG }, j}}\right)^{-1}+\sum_{j=1}^{m c}\left(\frac{1}{\Theta_{c o n, j}}\right)\right)^{-1}=\frac{T_{h, a v}-T_{c, a v}}{Q_{\text {in }}-\sum_{j=1}^{m}\left(I T_{h, j} \alpha_{m}+\frac{R_{T E G} I^{2}}{2}\right)}
\end{gathered}
$$

where $\Theta_{\text {con,j }}$ represents the aluminum conductors that replace the TEG modules in the primary heat path and $\mathrm{m}$ is the number of conductors in the array.

The third part is the thermal resistance in the cold side heat sink. The heat sinks dissipate heat from cold side of the array of TEG modules and are connected in parallel thermally

$$
\Theta_{e x}=\left(\frac{1}{\Theta_{e x, L}}+\frac{1}{\Theta_{e x, C}}+\frac{1}{\Theta_{e x, R}}\right)^{-1}=\left(\frac{1}{\frac{T_{c-L}-T_{a}}{Q_{c, 12}}}+\frac{1}{\frac{T_{c-C}-T_{a}}{Q_{c, 34}}}+\frac{1}{\frac{T_{c-R}-T_{a}}{Q_{c, 56}}}\right)^{-1}=\frac{T_{c, a v}-T_{a}}{Q_{i n}}
$$


The total resistance $\Theta_{\mathrm{th}, \mathrm{T}}$ is the sum of the thermal resistance between the device and ambient air:

$$
\Theta_{t h, T}=\Theta_{d-T E G}+\Theta_{T E G-a r a y}+\Theta_{e x}=\frac{T_{d}-T_{a}}{Q_{i n}}
$$

\subsection{Results and discussion}

\subsubsection{Fan performance in cold-side heat sink}

The pressure drop versus volume flow rate relation at a rated voltage of $12 \mathrm{~V}$ has been extracted from the data provided by the manufacturer[68]. Eq. 3.3.5 and 3.3.6 are used to construct a pressure drop versus volume flow rate curve at different voltage input to the fan.

The minimum voltage for the fan performance curve is constructed at $4.5 \mathrm{~V}$ which is the minimum voltage at which the fan moves. The maximum pressure (pressure at zero volume flow rate) decreases from around $38 \mathrm{~Pa}$ at $12 \mathrm{~V}$ to close to $5 \mathrm{~Pa}$ at $4.5 \mathrm{~V}$. Similarly, the maximum volume flowrate (flow rate at zero static pressure) decreases by an amount close to $65 \%$ as the voltage is reduced to $4.5 \mathrm{~V}$ from maximum rated voltage $(12 \mathrm{~V})$. To put the fan performance in context, the pressure drop at different volume flow rate has been simulated for the parallel plate-fin heat sink used in the experiment. The curve is superimposed on the fan performance curve to identify the fan operation point as shown in Figure 3-4. An experimentally derived equation relating the 
pressure drop at the heat sink, $\Delta \mathrm{Phk}$, with volume flow rate at the heat sink, $\dot{\mathrm{U}}_{\mathrm{hk}}$, for the parallel plate-fin heat sink in the wind tunnel is:

$$
\Delta P_{h k}=0.0015 \dot{U}_{h k}^{2}+0.0145 \dot{U}_{h k}+0.4714
$$

where $x_{l}$ is the length along the channel and $L_{c h}$ is the total length of the channel.

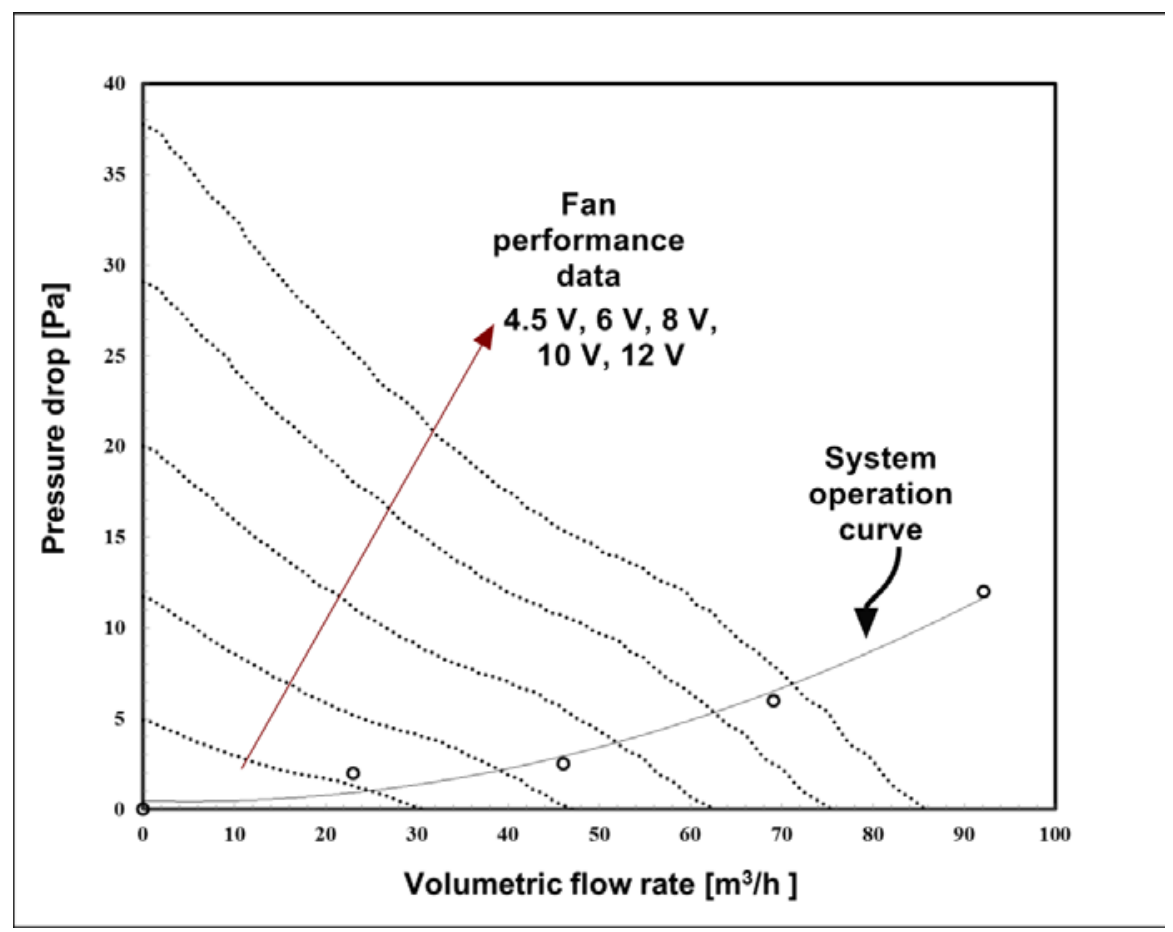

Figure 3-4 Fan-heat sink operation curve for case A and B

From Eq. 3.3.8, it could be determined that the fan operates at the minimum voltage of around $4.5 \mathrm{~V}$ and the minimum power supplied is around $0.4 \mathrm{~W}$. The maximum rated supply power to the fan at $12 \mathrm{~V}$ is around $1.7 \mathrm{~W}$. The internal resistance of the fan is approximately $72 \Omega$. The data helps in choosing the 
number and type of TEG modules which would be required to supply the required voltage and power to move the fan. For an array of TEG modules connected in series, the combined electrical resistance needs to be close to fan (load) internal resistance for optimal power transfer [69]. In the experimental set up the combined electrical resistance of the six TEG modules is $60 \Omega$ which is close to the fan's internal electrical resistance. Thus, the fan performance curve at variable voltage is important tool to analyze how the fan behaves in the selfcooling mode.

\subsubsection{Voltage and power in case $A$ and $B$}

As the voltage produced by TEG modules is directly proportional to the temperature difference across the TEG modules, it is worthwhile to examine the temperature variations at the hot and cold side of the TEG modules. The temperature variation at the hot side and cold side of the TEG module is shown in Figure 3-5. It could be observed that for all the heat inputs (70 -100 W), the hot side of the TEG at the primary heat path $(T C-h, 3)$ is higher that hot side of TEG module at the secondary heat path $(T C-h, 1)$. Similarly, the cold side of the TEG module at the primary heat path $(\mathrm{TC}-\mathrm{C}, 3)$ is also higher than cold side of the TEG module at the secondary heat path $(\mathrm{TC}-\mathrm{C}, 1)$. However, the temperature difference across the TEG modules in the primary heat path is on average 45-65 $\%$ higher for 70 to $100 \mathrm{~W}$ heat input respectively. Thus, the voltage produced at 
the TEG modules in secondary heat path would be less than TEG modules at the primary heat path in case $A$.
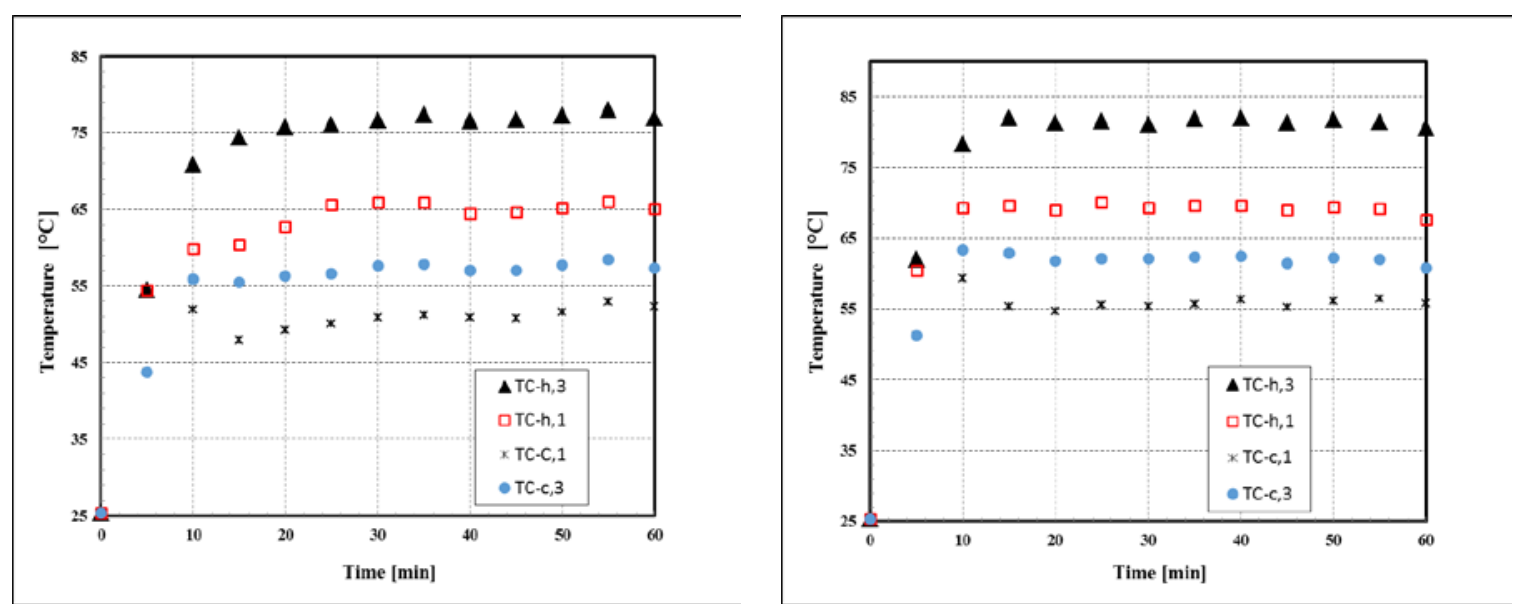

a)

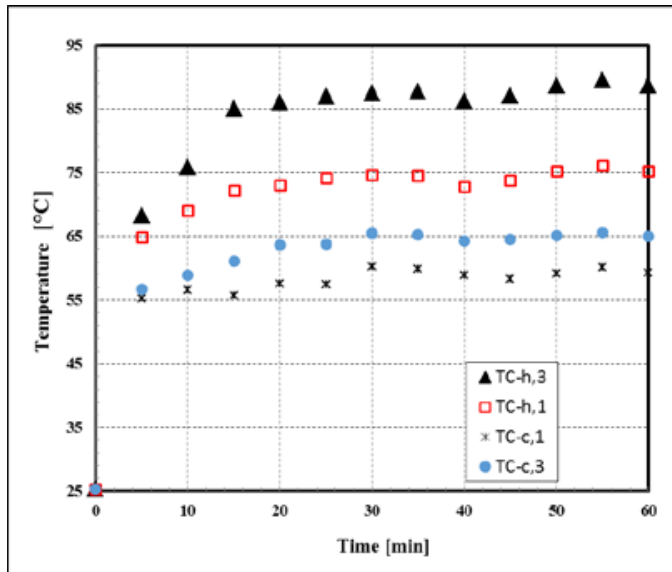

b)

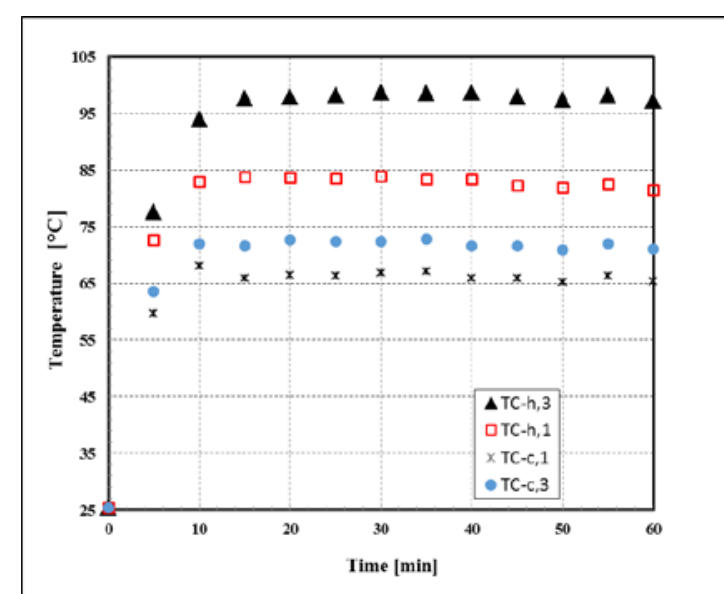

c)

d)

Figure 3-5 Temperature variations as a function of time for case A and Qin of a) $70 \mathrm{~W} \mathrm{b)} 80 \mathrm{~W} \mathrm{c)} 90 \mathrm{~W}$ and d) $100 \mathrm{~W}$

Figure 3-6 shows the variation of voltage $(V)$ as a function of heat input $Q_{\text {in }}$ at the device for case $A$. The voltage produced by the TEG \#1 and \#5 is represented by $V_{-} T_{E G}{ }_{1 / 5}$ while the sum of voltage generated by TEG \#2 and \#6 is given as 
$V_{-} T E G_{2 / 6}$. Both indicate the voltage produced temperature difference in the secondary heat path. The sum of voltage by TEG \#3 and \#4 (V_TEG $\left.{ }_{3 / 4}\right)$ represents the voltage from TEGs in the primary heat path. The total voltage from all the TEGs in the array is depicted $V_{-} T E G$ total.

It could be observed that for voltage has increased steadily with heat input both in primary and secondary heart paths. As heat input in the device rises, the temperature difference across the TEG module surfaces grows. This, in turn, results in rise in voltage generation. However, the voltages in $V_{-} T E G_{1 / 5}$ and $\mathrm{V}_{-} \mathrm{TEG}_{2 / 6}$ are on average $55-65 \%$ of $\mathrm{V}_{-} \mathrm{TEG}_{3 / 4}$. This is due to decreases in hot side temperature on TEG surfaces on the secondary heat path. The major part of the heat transfer occurs in the primary heat path. Thus, the estimation of voltage produced by TEG needs to consider the relative area of heat source with the TEG array surface area. Although increasing the number of TEGs results in more voltage generation, the effectiveness of the method decreases as the area of TEG increases relative to the heat source area. The magnitude of $V_{-} T E G_{1 / 5}$ is slightly more than $\mathrm{V}_{-} T E G_{2 / 6}$ as the TEG are located on the front side with respect to air flow which increases the temperature difference across the TEG surfaces.

Generally, it is observed from Figure 3-6a that for a given heat input, the $V_{-}{ } G_{\text {total }}$ rises until it achieves the fan's start-up voltage $\left(V_{s-u p}\right)$. For the fan used in the experiment, $V_{\text {s-up }}$ is experimentally determined to be around 4.5 $\pm 0.05 \mathrm{~V}$. Once the fan starts rotating, the voltage required to sustain the 
minimum rotating speed decreases. The fan runs as long as its voltage is higher than minimum (stall) voltage. In case A, the experimental result indicated that the minimum heat input $\left(\mathrm{Q}_{\mathrm{in}, \mathrm{cr}}\right)$ to achieve $\mathrm{V}_{\mathrm{s} \text {-up }}$ is about $\mathrm{Q}_{\mathrm{in}}=60 \mathrm{~W}$. The time taken to attain $\mathrm{V}_{\text {s-up }}\left(\mathrm{t}_{\mathrm{min}}\right)$ is recorded to be 11 minutes. At $\mathrm{Q}_{\text {in }}=120 \mathrm{~W}$, $\mathrm{t}_{\text {min }}$ decreases to 2 minutes. For $\mathrm{Q}_{\text {in }}$ less than $60 \mathrm{~W}$, the system could potentially develop voltage that sustains minimum speed but falls short of achieving $V_{\text {s-up. }}$. As the heat is augmented from $60 \mathrm{~W}$ to $130 \mathrm{~W}$, the steady state value of $V_{-} T E G_{\text {total }}$ increases by around $89 \%$. As shown in Figure 3-6, the current produced by the TEG array rose from $69.50 \mathrm{~mA}$ to close to $130 \mathrm{~mA}$ for the heat increase from $60 \mathrm{~W}$ to $130 \mathrm{~W}$ while the power produced per area of TEG array (P/ATEG) also increased by almost $300 \%$ to close to $10.7 \mathrm{~W} / \mathrm{cm}^{2}$.

For case B (Figure 3-7), all the TEGs are placed on secondary heat path and the magnitude of $V_{-} T_{E G} / 5$ is slightly higher (around $7 \%$ ) than $V_{-} T E G_{2 / 6}$. This is again due to placement of $V_{-} T E G_{1 / 5}$ at the front side with the air flow which helps with temperature difference across the modules. For the same heat input, the total voltage produced in Case $B$ is $50-70 \%$ of the values in Case $A$. The decreases could be attributed to the fact that, in case B, the voltage is only produced by the TEG in the secondary heat path.

As $Q_{\text {in }}$ is increased from $60 \mathrm{~W}$ to $130 \mathrm{~W}, \mathrm{~V}_{-}$TEG total rise by around $160 \%$. However, the percentage increase for $V_{-} T E G_{\text {total }}$ for a rise in $Q_{\text {in }}$ from $90 \mathrm{~W}$ to $130 \mathrm{~W}$ is only $20 \%$ as compared to $115 \%$ for $\mathrm{Q}_{\text {in }}$ from $60 \mathrm{~W}$ to $90 \mathrm{~W}$. When self- 
cooling starts at $90 \mathrm{~W}$, forced convection decreases the cold side temperature of the TEG. This results in a sudden climb of the value of $\mathrm{V}$. For a rise in $\mathrm{Q}_{\text {in }}$ after the $\mathrm{Q}_{\mathrm{in}, \mathrm{cr}}$, the system shows a steady increase in $\mathrm{V}_{-} T E \mathrm{G}_{\text {total }}$ which results in a steady temperature rise in the device. At $\mathrm{Q}_{\text {in }}=130 \mathrm{~W}$, case B produces $33 \%$ and $56 \%$ less voltage and power than case A Nevertheless, it is effective in power production per area of array of TEG modules. For the same heat input, case B generates P/A teg $\left(9.61 \mathrm{~mW} / \mathrm{cm}^{2}\right)$ which is only slightly lower than case A (10.79 $\left.\mathrm{mW} / \mathrm{cm}^{2}\right)$.

\subsubsection{Dynamic thermal response case $A$ and $B$}

It could be inferred from Figure 3-8 that at $\mathrm{Q}_{\text {in }}=50 \mathrm{~W}$, the temperature of device above room temperature $\left(\Delta \mathrm{T}_{\text {de }}\right)$ climbs rapidly to around $63.29{ }^{\circ} \mathrm{C}$ within 60 minutes. As the fan is not rotating below $\mathrm{Q}_{\mathrm{in}, \mathrm{cr}}$ of $60 \mathrm{~W}$, the heat loss mechanism is natural convection via the dissipater. However, once the fan starts rotating at $60 \mathrm{~W}$, the temperature drops by close to $17^{\circ} \mathrm{C}$ from its value at $50 \mathrm{~W}$. In addition, the slope of rise of temperature versus time also decreases enabling the system to stay at steady temperature after 12 minutes. 
a)
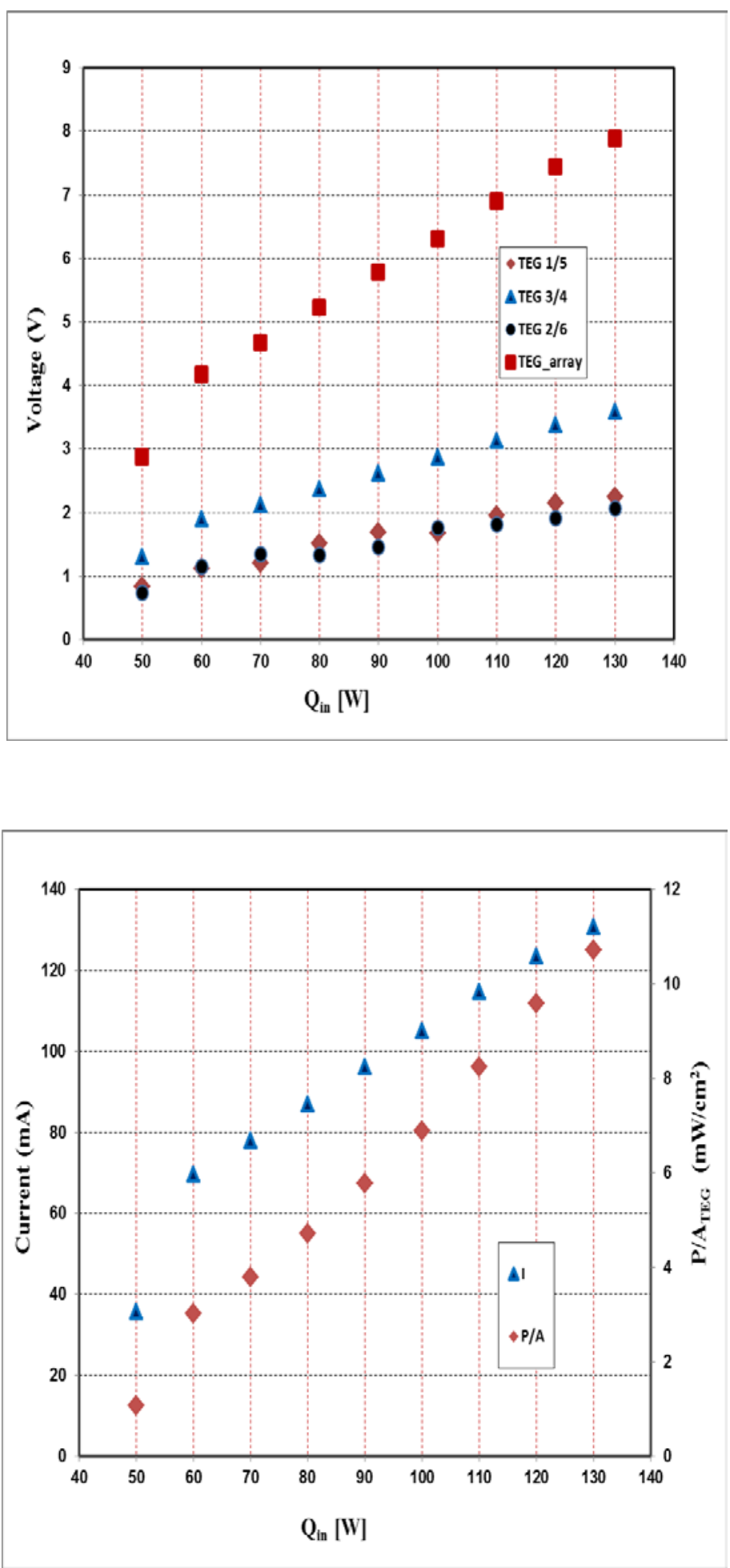

Figure 3-6 a) Total array Voltage and b) Power per unit area of TEG (PIATEG) and I as a function of Qin for case A 


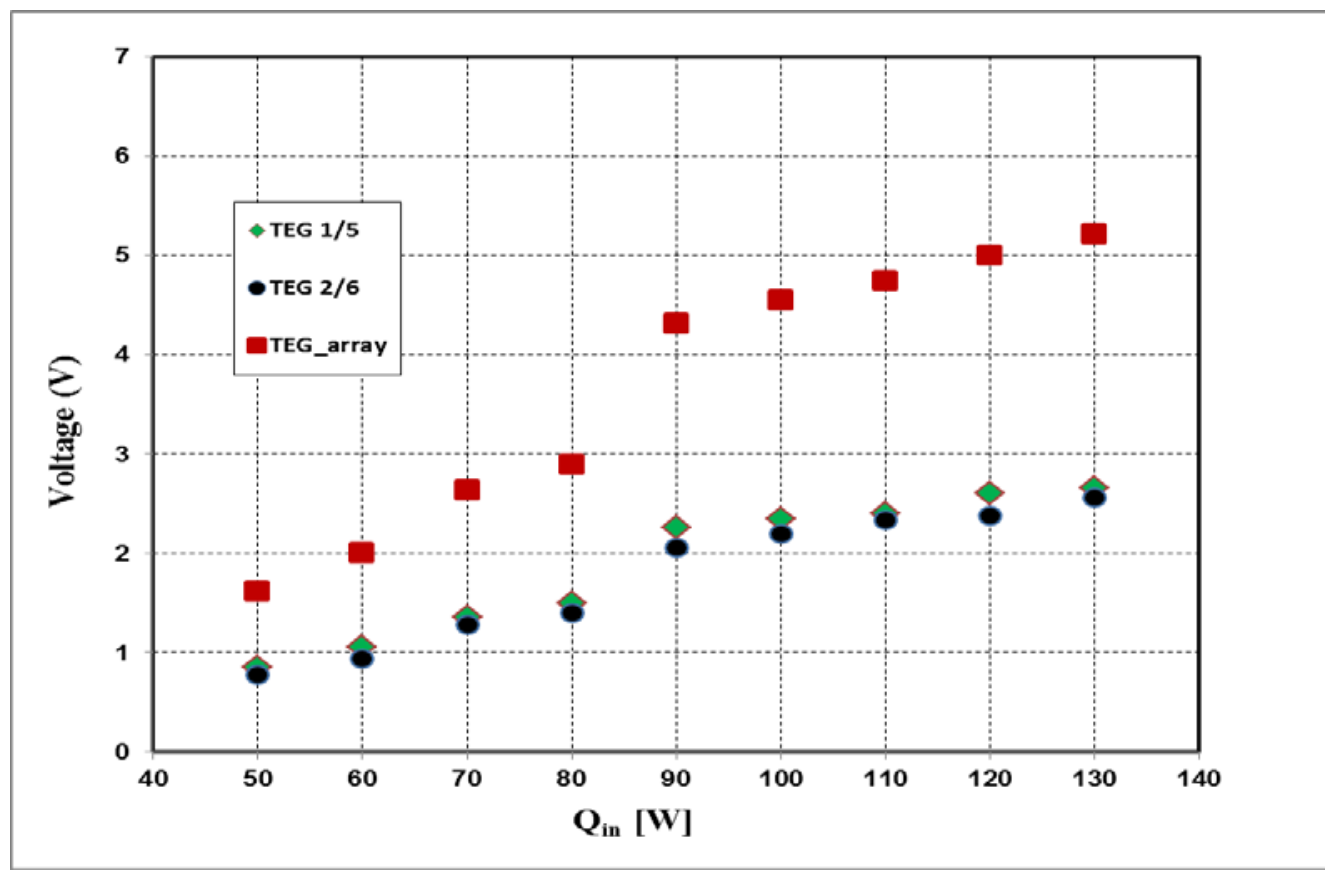

\section{a)}

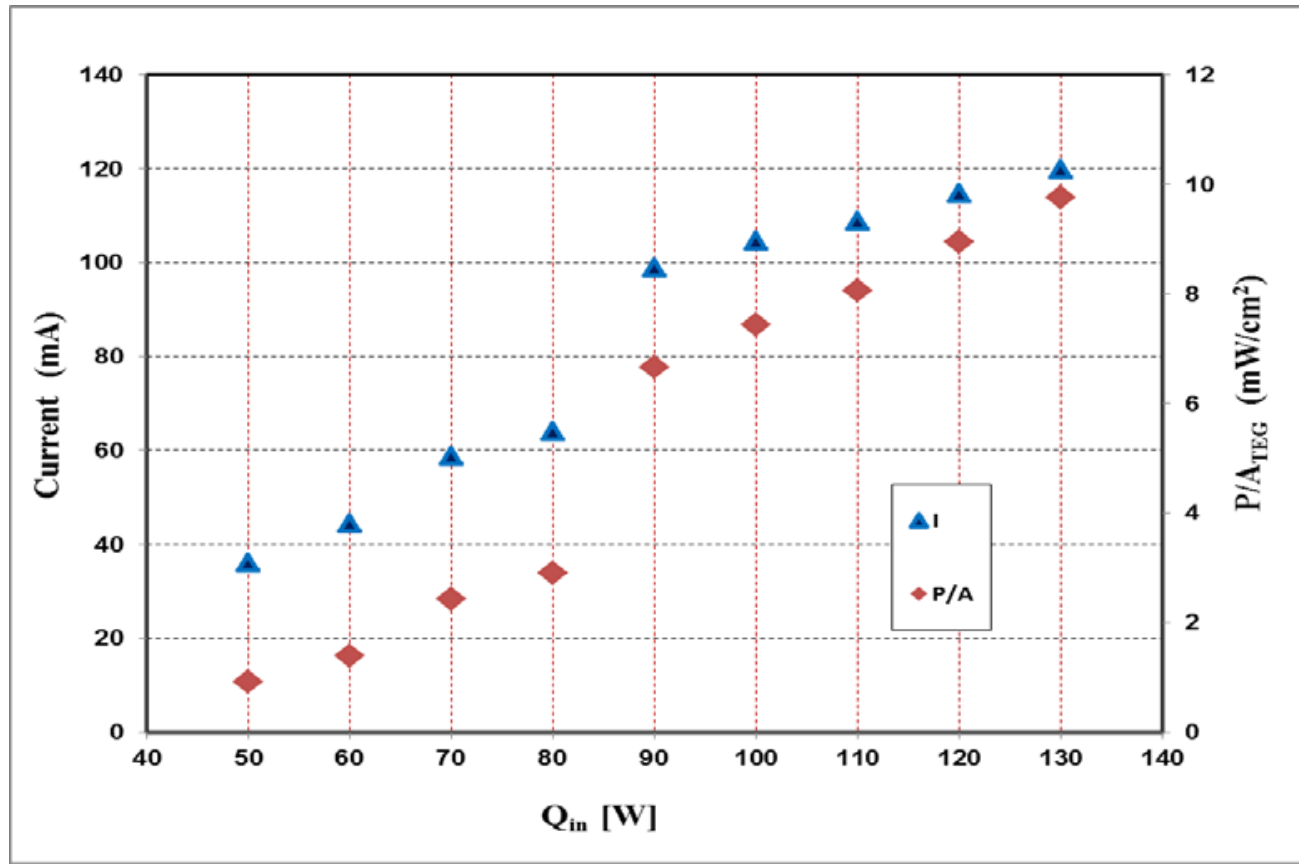

b)

Figure 3-7 a) Total array Voltage and b) Power per unit area of TEG (P/ATEG) and Current as a function of Qin for case B 
Figure 3-8 also shows that at $\mathrm{Q}_{\text {in }}$ of $80 \mathrm{~W}$, the maximum temperature of $\mathrm{T}_{d}$ after an hour is $4{ }^{\circ} \mathrm{C}$ less than $\mathrm{Q}_{\text {in }}(50 \mathrm{~W})$. For $\mathrm{Q}_{\text {in }}$ ranging from $90-120 \mathrm{~W}, \Delta \mathrm{T}_{\text {de }}$ rises only by $24^{\circ} \mathrm{C}$. As self-cooling allows for steady forced air convection cooling, the rise of temperature has been steady.

As shown in Figure $3-9, \Delta \mathrm{T}_{\text {de }}$ after one hour climbs to $60^{\circ} \mathrm{C}$ and $79.8^{\circ} \mathrm{C}$ for heat input of $60 \mathrm{~W}$ and $80 \mathrm{~W}$. In case $\mathrm{B}$, the system achieves $\mathrm{Q}_{c r, i n}$ at $90 \mathrm{~W}$. At $\mathrm{Q}_{\text {cr, in }}$ ,the fan starts to move in around 16 minutes from rest and $\Delta T_{\text {de }}$ decreases to around $64^{\circ} \mathrm{C}$.

For heat input more than $\mathrm{Q}_{c r, i n}$, the system functions in self-cooling mode with the fan rotating in less than 8 minutes from rest. The steady state temperature $T_{d}$ increases only by $11.8^{\circ} \mathrm{C}$ for $30 \mathrm{~W}$ rises in $\mathrm{Q}$ in from $100 \mathrm{~W}$. The rate of increases is almost $50 \%$ less than case A. This is due to combined effect of self-cooling (less convection resistance) and reduced conduction resistance.

In addition, the slope of rise of temperature versus time also decreases enabling the system to stay at steady temperature after 12 minutes. Figure 3-8 also shows that at $\mathrm{Q}_{\text {in }}$ of $80 \mathrm{~W}$, the maximum temperature of $\mathrm{T}_{\mathrm{d}}$ after an hour is $4{ }^{\circ} \mathrm{C}$ less than $\mathrm{Q}_{\text {in }}(50 \mathrm{~W})$. For $\mathrm{Q}_{\text {in }}$ ranging from $90-120 \mathrm{~W}, \Delta \mathrm{T}_{\text {de }}$ rises only by $24^{\circ} \mathrm{C}$. As self-cooling allows for steady forced air convection cooling, the rise of temperature has been steady. 
As shown in Figure $3-9, \Delta \mathrm{T}_{\text {de }}$ after one hour climbs to $60^{\circ} \mathrm{C}$ and $79.8^{\circ} \mathrm{C}$ for heat input of $60 \mathrm{~W}$ and $80 \mathrm{~W}$. In case $\mathrm{B}$, the system achieves $\mathrm{Q}_{\mathrm{cr}, \mathrm{in}}$ at $90 \mathrm{~W}$. At $\mathrm{Q}_{\mathrm{cr}, \mathrm{in}}$ ,the fan starts to move in around 16 minutes from rest and $\Delta T_{\text {de }}$ decreases to around $64{ }^{\circ} \mathrm{C}$. For heat input more than $\mathrm{Q}_{\text {cr,in, }}$, the system functions in self-cooling mode with the fan rotating in less than 8 minutes from rest.

The steady state temperature $T_{d}$ increases only by $11.8^{\circ} \mathrm{C}$ for $30 \mathrm{~W}$ rises in $Q_{\text {in }}$ from $100 \mathrm{~W}$. The rate of increases is almost $50 \%$ less than case $\mathrm{A}$. This is due to combined effect of self-cooling (less convection resistance) and reduced conduction resistance.

\subsubsection{Application of varying load in case $B$}

In practical applications, the heat generation in a device could vary as a function of time. Thus varying heat loading scenarios has been tested and their effect on the device temperature and voltage produced by array of TEG modules is observed as shown in Figure 3-10.

The first scenario (S1) is gradual heating in which heat input is increased by 10 W every 15 minutes starting from $100 \mathrm{~W}$. In the second scenario (S2), the heat input is decreased from $130 \mathrm{~W}$ by $10 \mathrm{~W}$ every 15 minutes to represent gradually decreasing thermal loading. 
Third scenario (S3) is a case in which the system enters no thermal loading cases at varying instants. In this scenario,150 $\mathrm{W}$ is applied in the first 10 minutes, and a brief no thermal loading for the next 2 minutes followed by another 10 minutes of heating at $150 \mathrm{~W}$. This is again followed by 5 minutes of no loading, 10 minutes of $150 \mathrm{~W}$ loading and 10 minutes of no thermal load.

In S1, the fan starts to turn approximately around 9 minutes and the system achieves a steady temperature increase to $98^{\circ} \mathrm{C}$ at the15th minute. When the heat input is ramped up by $10 \mathrm{~W}, \mathrm{Td}$ rose by $9{ }^{\circ} \mathrm{C}$ in the next 15 minutes. For the heat load increase of $10 \mathrm{~W}$ from $110 \mathrm{~W}$ to $120 \mathrm{~W}$ and $120 \mathrm{~W}$ to $130 \mathrm{~W}$, the temperature increases was only $7^{\circ} \mathrm{C}$ and $6^{\circ} \mathrm{C}$ at the third rise to $130^{\circ} \mathrm{C}$.

This indicates that once the system achieves a steady run, a gradual intensification of heat input resulted only with slight increases in temperature. Similarly with S2, with higher input of $130 \mathrm{~W}$, the temperature starts to rise steeply after which, the slope decreases and temperature stabilizes. After that a slow fall in temperature is observed with a gradual decrease in heat input. 


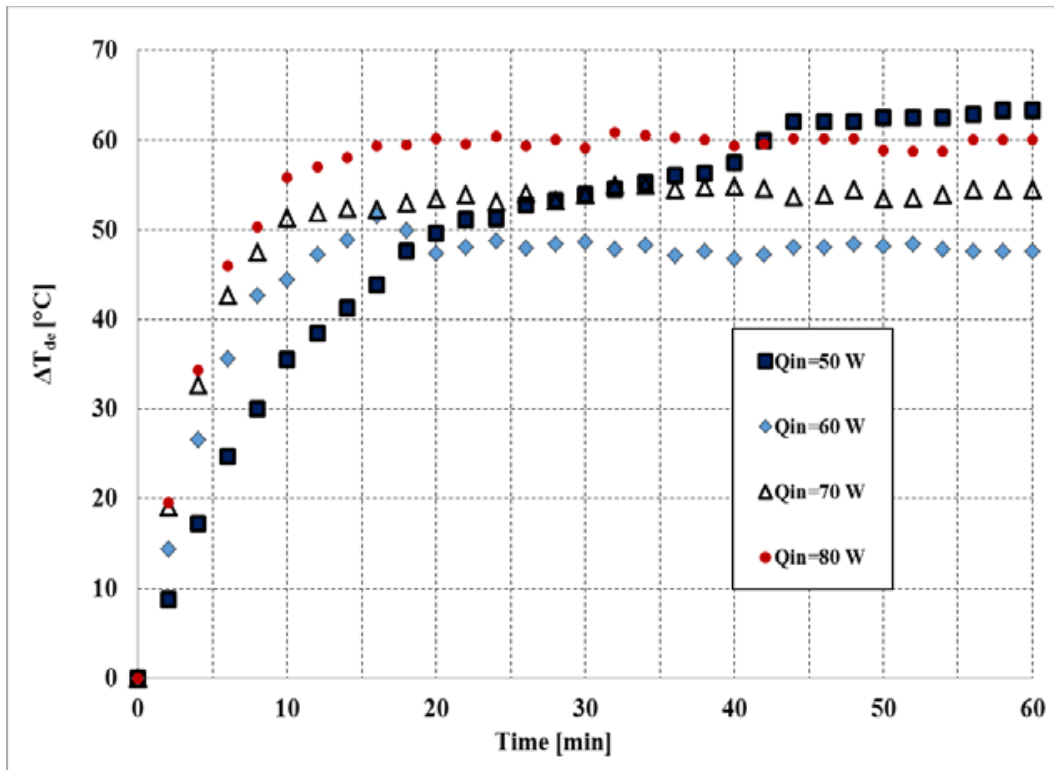

a)

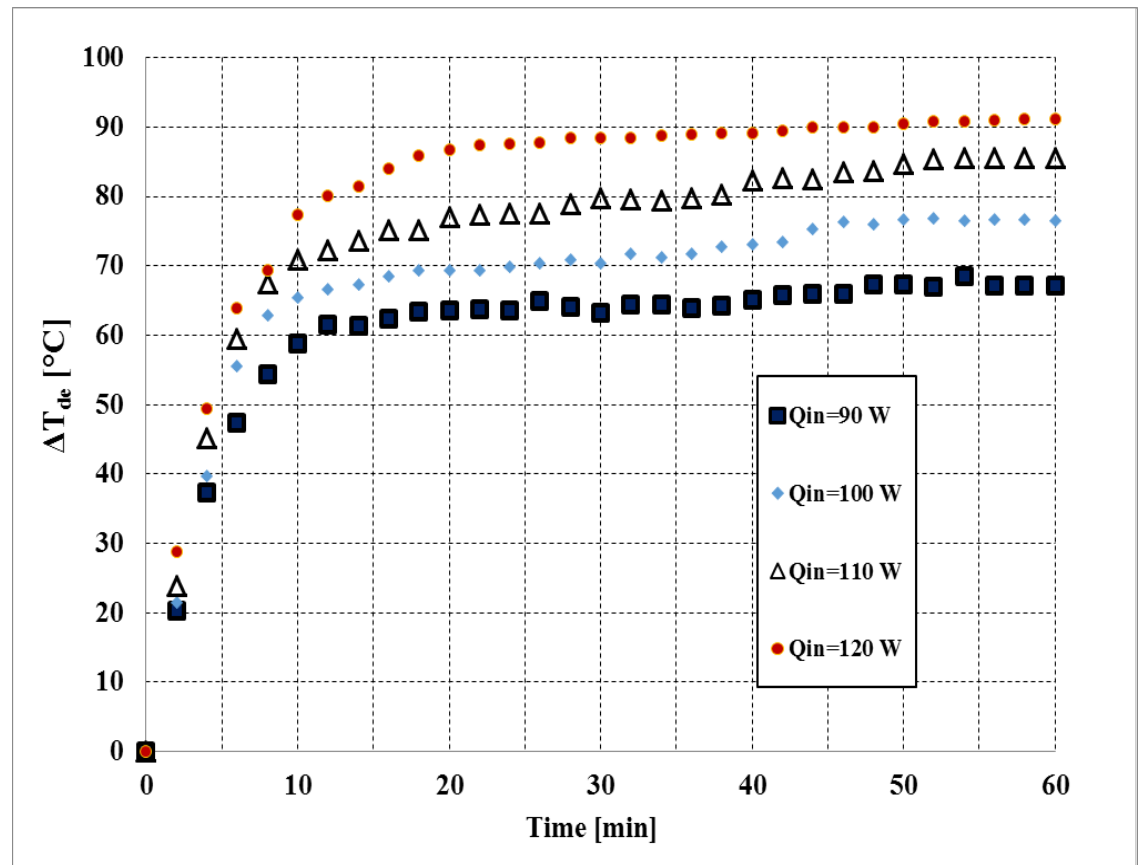

b)

Figure 3-8 Temperature difference $\left(\Delta T_{d e}\right)$ between the device temperature $\left(T_{d}\right)$ and environment temperature $\left(\mathrm{Te}_{\mathrm{e}}\right)$ for case $\mathrm{A}$ at varying heat input $\left(\mathrm{Q}_{\text {in }}\right)$ of $\left.a\right)$ $50-80 \mathrm{~W}$ b) $90-120 \mathrm{~W}$ 


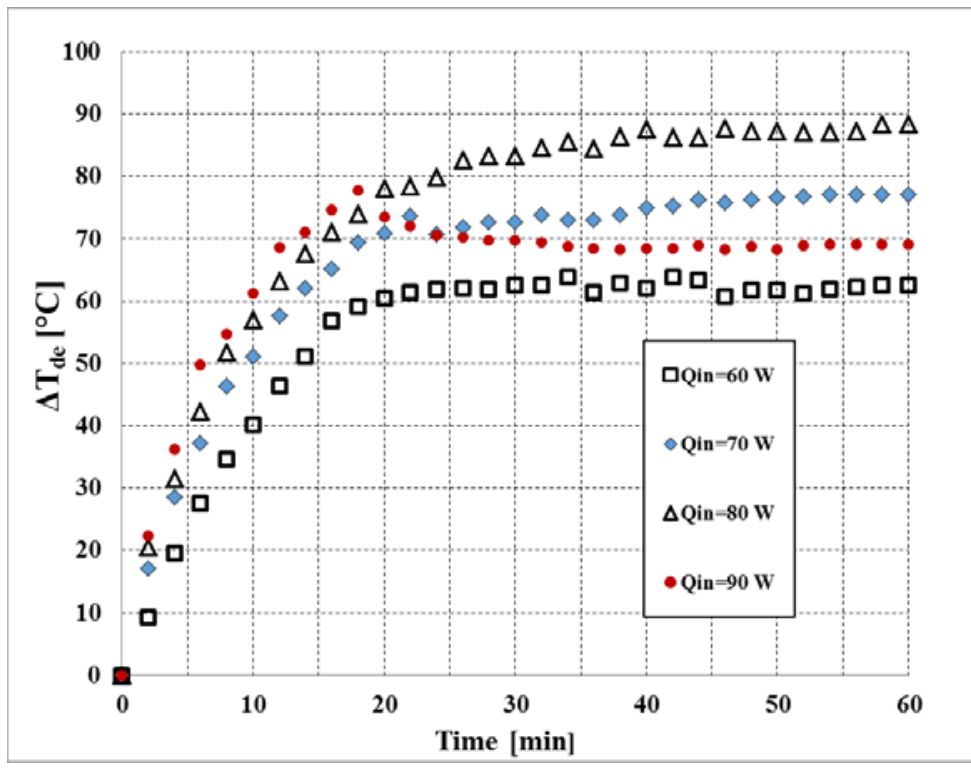

a)

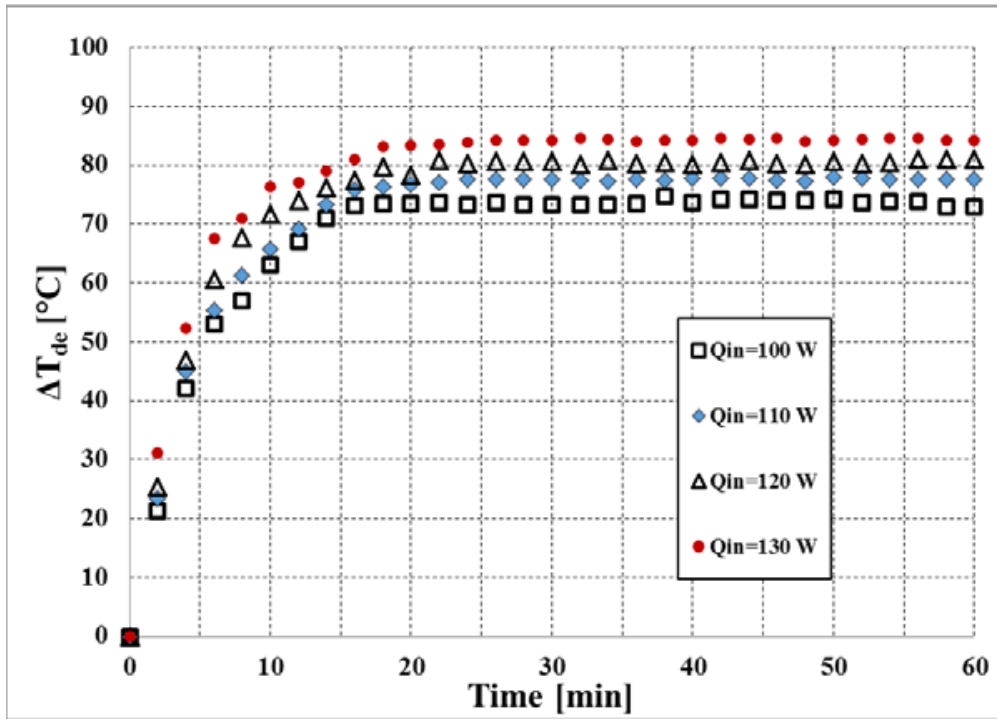

b)

Figure 3-9 Temperature difference $\left(\Delta T_{d e}\right)$ between the device temperature $\left(T_{d}\right)$ and environment temperature $\left(T_{e}\right)$ for case $B$ at varying heat input $Q_{\text {in }}$ of a) $60-90 \mathrm{~W}$ b) 100-130 W 
S3 represents a scenario where there is a sudden steep fall or surge in thermal loading. In the first 10 minutes $\mathrm{Td}$ reaches a steady state within 10 minutes as the fan starts to turn at 3 minutes and temperature stabilizes shortly after. In the second stage, the power input has been throttled to zero and the temperature decreases due to no thermal loading as well as forced convection from the fan. The fan continues to rotate due to the residual temperature difference in the system.

A sudden ramp up of heat input to $150 \mathrm{~W}$ in the third and fifth stage has been handled well with the system achieving a steady temperature in 5 minutes. As the load is suddenly augmented, the voltage input to the fan grows resulting in increased forced convection.

\subsubsection{Comparison between case a-c}

The thermal performance of different cases could be analyzed by analyzing the total device to ambient thermal resistance $\left(\Theta_{\mathrm{th}, \mathrm{T}}\right)$. As Figure $3-11 \mathrm{a}$ shows, the total thermal resistance changes markedly for all the cases when the heat input reaches $\mathrm{Q}_{\mathrm{cr}}$. For case $\mathrm{A}, \Theta_{\mathrm{th}, \mathrm{T}}$ reduces by $40 \%$ for $\mathrm{Qin}$ reached $60 \mathrm{~W}$ due to the increase in convection heat transfer coefficient and the temperature of the device decreased by $18 \%$.

After $60 \mathrm{~W}$, the total thermal resistance only slightly decreases as the system has achieved forced convection and the fan velocity only changed marginally. The 
temperature of the device only increases by $61 \%$ for a rise in heat input from 60 W to $120 \mathrm{~W}$ (Figure $3-11 \mathrm{~b}$ ). In case A, device temperature was $25^{\circ} \mathrm{C}$ less than case C for Qin=100 W which increases to over $34{ }^{\circ} \mathrm{C}$ at $130 \mathrm{~W}$.

For heat input of $130 \mathrm{~W}$, case $\mathrm{A}$ has $30 \%$ less thermal resistance as compared to case C.Similar effect was observed for case B after $90 \mathrm{~W}$. From $80 \mathrm{~W}$ to $90 \mathrm{~W}$, there is a $15 \%$ drop of temperature while there is only a $16 \%$ increase in temperature as the heat input is increased by $40 \mathrm{~W}$ from $90 \mathrm{~W}$. Case B has been able to reduce $\mathrm{Td}$ by $16{ }^{\circ} \mathrm{C}$ and $40{ }^{\circ} \mathrm{C}$ for the same heat input of $120 \mathrm{~W}$ as compared to case A and C respectively. Case B has $40 \%$ less thermal resistance than case $\mathrm{C}$ for heat input of $130 \mathrm{~W}$ at the device.

Case $\mathrm{C}$ has more or less constant thermal resistance which is mainly due to natural convection from surrounding air. For heat input less than Qcr, Case A has $18 \%$ more resistance due to the presence of TEG modules in the heat path while case $B$ has comparable thermal resistance with case $C$ even when the fan is not moving. Therefore case B has dual advantages as compared to case A by reducing the thermal resistance when the system is both in self-cooling and natural convection mode. 

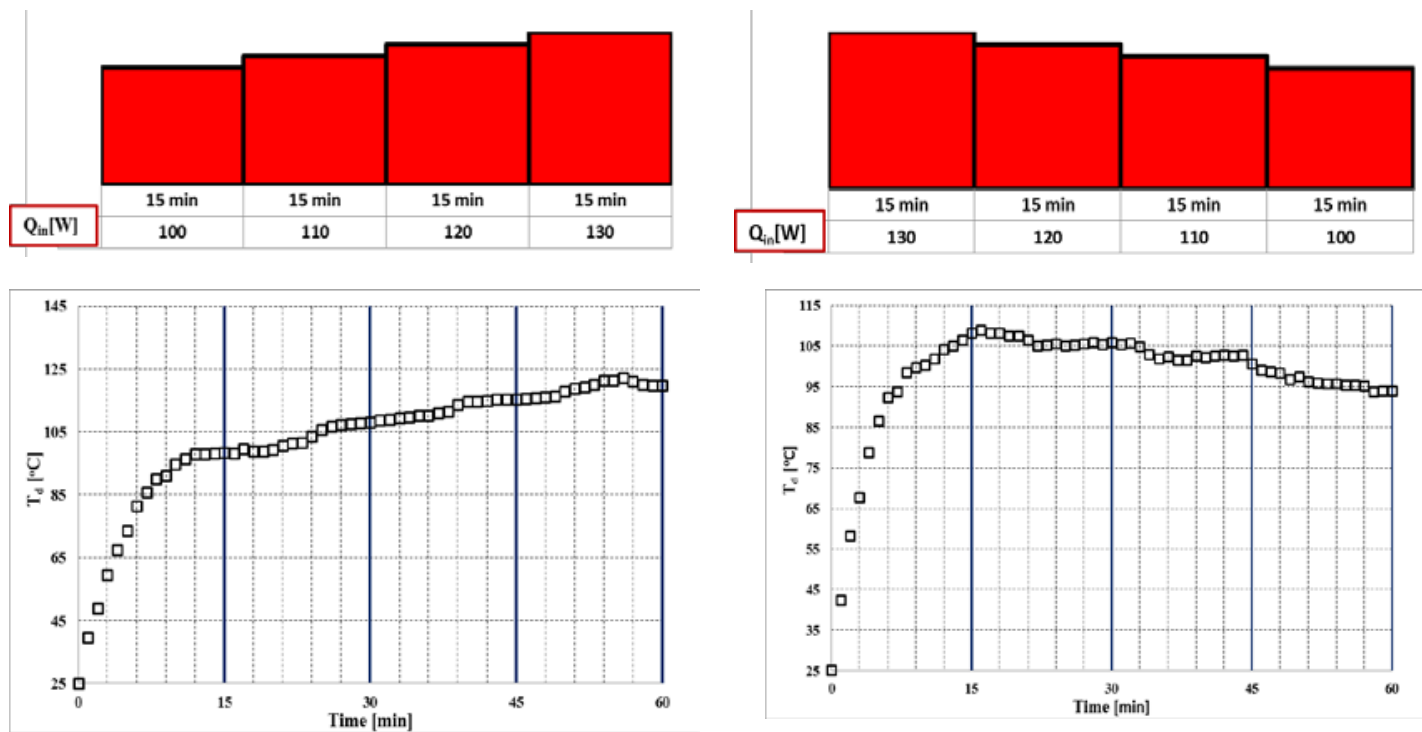

a)

b)
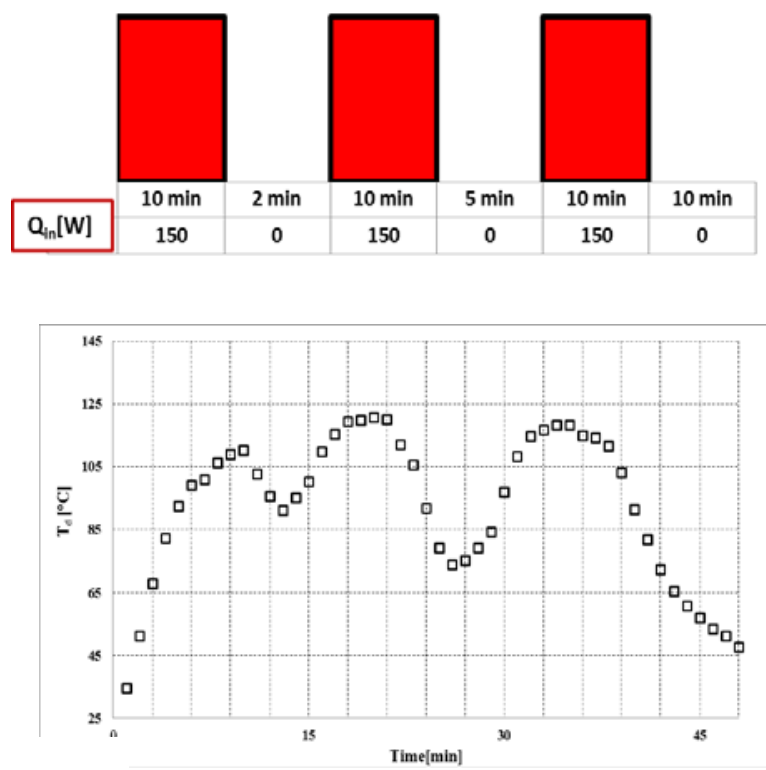

c)

Figure 3-10 Variation of device temperature (Td) for varying heat input load (Qin) a) S1 b) S2 c) S3 
Figure 3-12 displays the percentage of magnitude of contribution of thermal resistance of parts of the total device set-up as compared to the total resistance of the whole system for case A and B. It could be expressed by the equation:

$$
\Delta \Theta \%=\frac{\Theta_{i}}{\Theta_{t h, T}}
$$

where $\Theta_{i}$ is the thermal resistance of part of the device and $\Theta_{\mathrm{th}, \mathrm{T}}$ is the total resistance of the model. The figure represents the three major parts $\Theta_{d-T E G}$ in Eq.3.3.12, $\Theta_{\text {TEG-array }}$ in Eq.3.3.16, Eq.3.3.17and $\Theta_{e x}$ in Eq. 3.3.18.

It could be inferred from Fig. 12 that the $\Theta_{\mathrm{d}-\mathrm{TEG}}$ is responsible on average for onequarter of the total thermal resistance for both case $A$ and $B$. In case A, $\Theta_{\text {TEG-array }}$ amounted to about $30 \%$ of the total resistance from $60 \mathrm{~W}$ to $120 \mathrm{~W}$.

Its effect is almost equivalent to $\Theta_{d-\text { TEG }}$ in case $A$ which is indicative of the fact

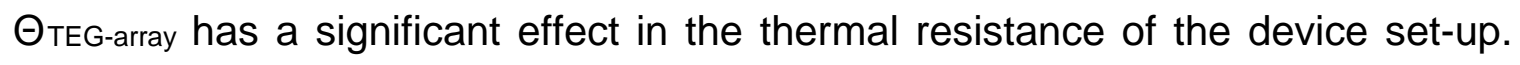
While for case $B$, the ratio of $\Theta_{d-T E G}$ in $\Theta_{t h, T}$ has been significantly reduced. Replacing the TEG modules by aluminum block in the primary heat path has resulted in such important improvement. 


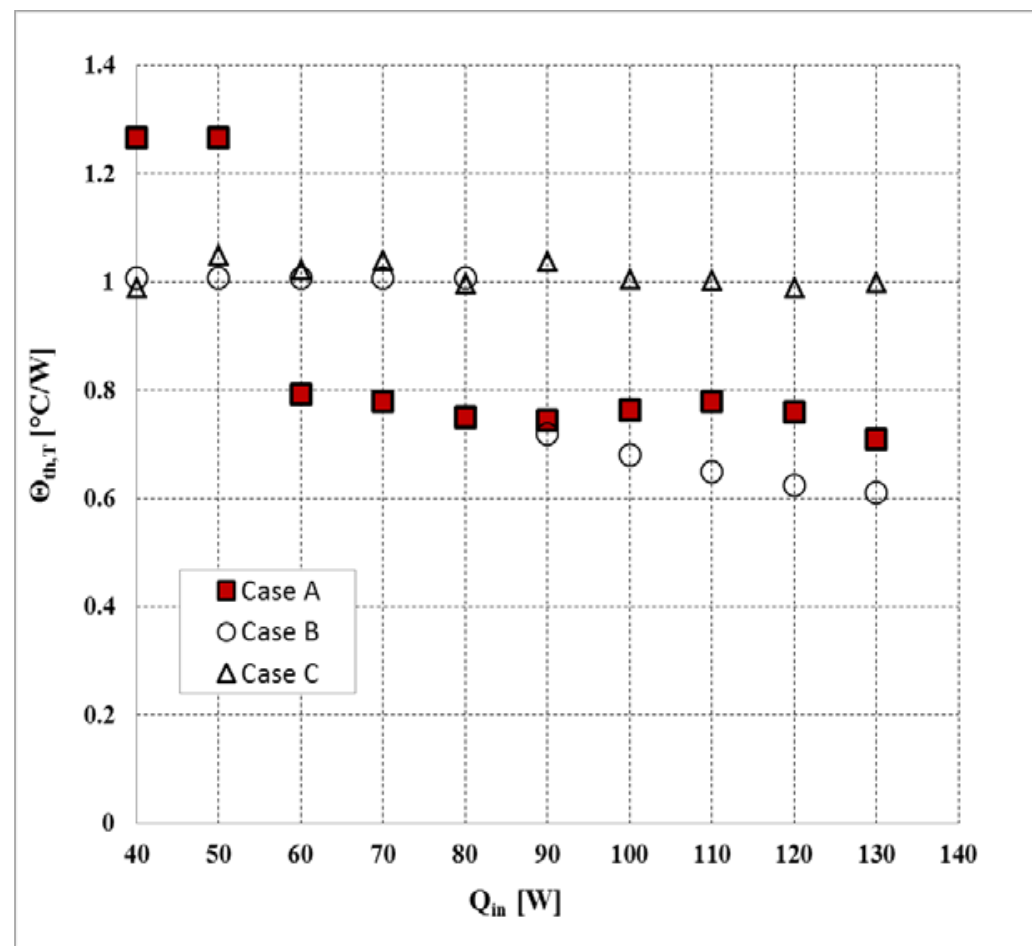

a)

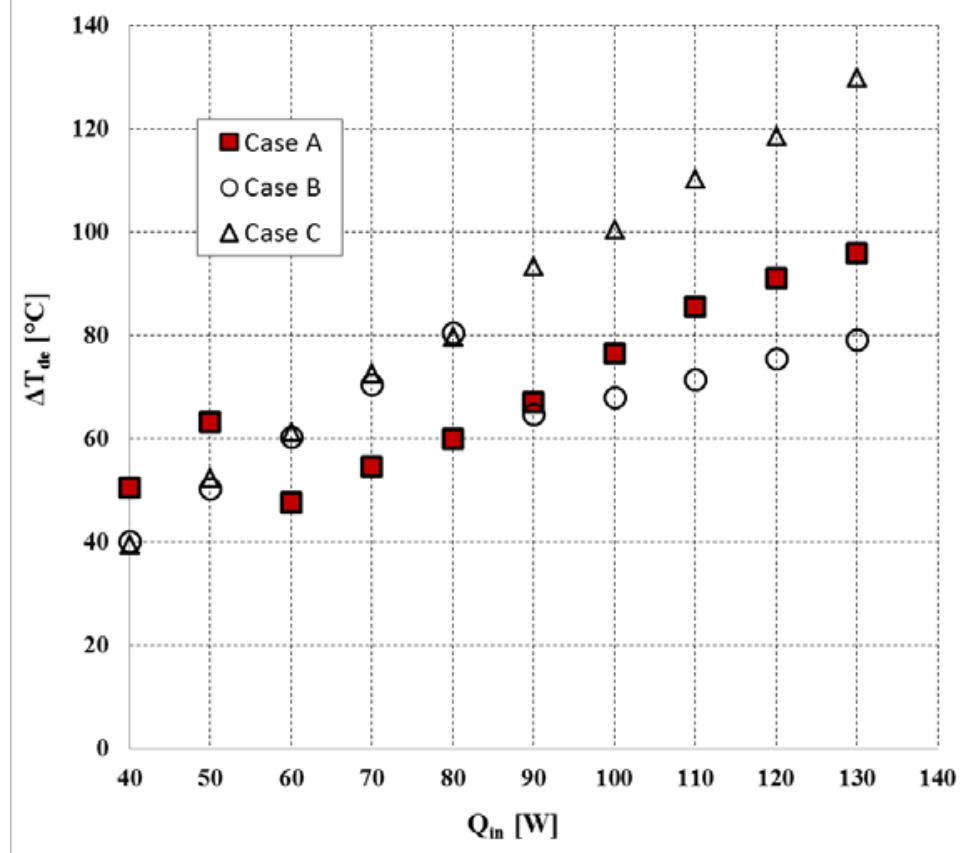

b)

Figure 3-11 Variation of a) Total thermal resistance, $\Theta_{\mathrm{th}, T}$ b) $\Delta \mathrm{T}_{\mathrm{de}}$ 


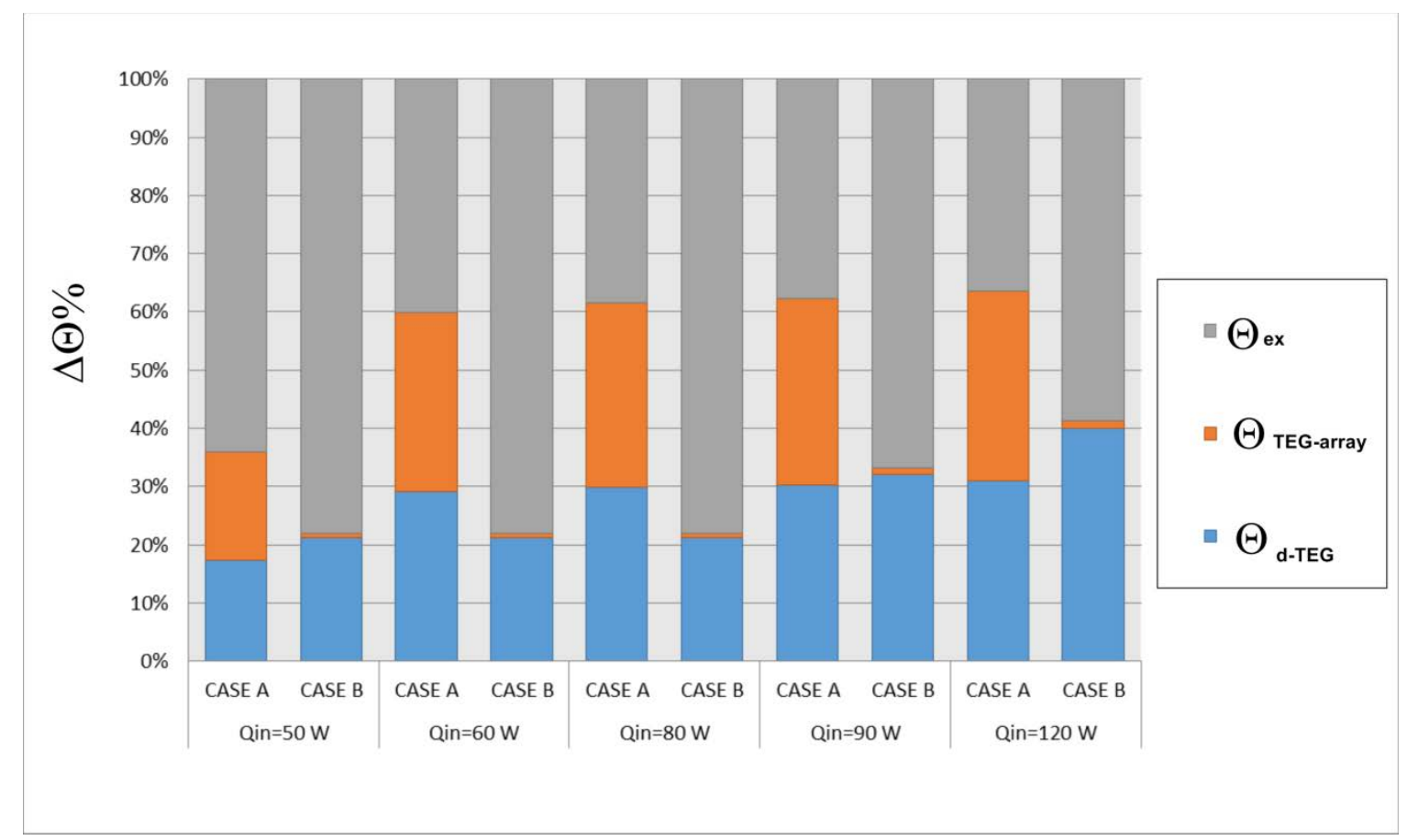

Figure 3-12 Comparison of the percentage ratio, $\Delta \Theta \%$

$\Delta \Theta_{\mathrm{ex}} \%$ has slightly decreased at $\mathrm{Q}_{\mathrm{in}, \mathrm{cr}}(90 \mathrm{~W})$ with the start of the fan and increased convection thermal resistance. Nevertheless, it still is responsible for almost $60 \%$ of the total thermal resistance. As case B has been able to reduce OTEG-array significantly, further improvement can be achieved by minimizing $\Theta_{e x}$ 


\section{NUMERICAL SIMULATION METHODS ON SELF-COOLING SYSTEM}

\subsection{Introduction}

Although there have been many studies focusing on the general modeling of TEGs coupled with fluid models [70][71][72][73][74][75][76]], there has only been very limited research with respect to numerically modeling TEG based selfcooling application[77]. There are some peculiar factors pertinent to the modeling of self-cooling incorporating TEG modules. Firstly, the system has an internal loop in power generation. TEG modules provide the power for the fan/pump that cools the modules.

Thus, an inlet condition for fluid flow into the cold side heat sink is coupled with the voltage and power generated from the modules. Secondly, TEG modules provide a variable voltage depending on temperature difference across the modules, thus, the fan/pump provide variable speed water/air flow (volume flow rate). Thirdly, as the TEG modules are placed between the heat source device

and heat sink, there is a need for numerical modeling that helps to study the minimization of thermal resistance while providing enough temperature difference to power the cooling systems.

Thus, in this study, a general numerical methodology has been proposed for the study of self-cooling application. The numerical computational strategy could be solved in any appropriate software or could be coded in a relevant programming 
language. However, the use of dedicated computational fluid dynamics (CFD) software with ability to integrate user defined functions ensures various complexities of heat sink and device set up could be readily handled in SFF and $\mathrm{SHT}$. The modeling strategy has been implemented in Finite Element model (FEM) and Finite volume method (FVM) models.

The numerical model is validated in two steps. In the first step, the numerical model is implemented in FEM model and validated against existing experimental data. In the second step, a finite volume model is implemented and validated with own experimental data from chapter 3.

\subsection{Numerical methodology}

\subsubsection{Voltage and power}

A general numerical methodology is developed which could be utilized to analyze TEG based self-cooling systems as shown in Figure 4-1. There are three submodels that constitute the general structure of the numerical methodology namely submodel: fluid flow (SFF), submodel: conjugate heat transfer (SHT) and submodel: electrical circuit (SEC).

Submodel fluid flow (SFF) represents three dimensional fluid flow analyses in the fan (pump) - heat sink model. There are some important characteristics to consider when pressure drop for self-cooling applications are considered. 


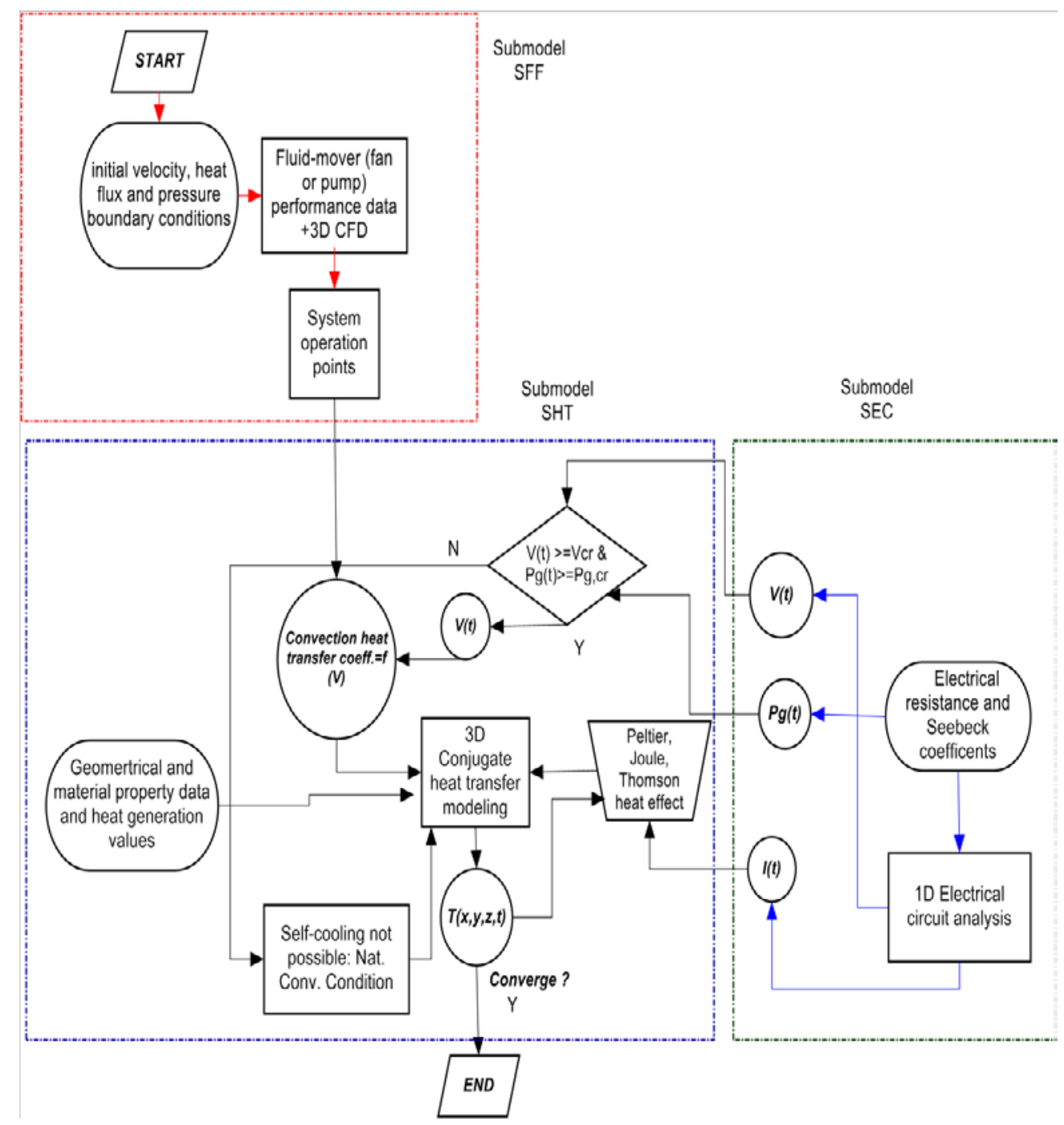

Figure 4-1 Numerical Methodology

The fan/pump operating points are defined at the intersection points between the fan/pump performance curves and system resistance curves. Fan/pump manufactures usually test and provide performance curves at a rated voltage. However, the characteristic of self-cooling applications is that the voltage 
supplied to the fan/pump is variable and could be different from rated voltage. It is, therefore, necessary to construct a pressure drop/ volume flow rate performance curve for the fan/pump at different voltages.

The pressure drop versus volume flow rate relation at a rated voltage of $12 \mathrm{~V}$ has been extracted from the data provided by the manufacturer[68]. Eq. 3.3.5 and 3.3.6 are used to construct a pressure drop versus volume flow rate curve at different voltage input to the fan.

Once the fan performance points at different voltage are derived using the relations mentioned above, the 3D fluid flow analysis is utilized to construct appropriate boundary conditions in two steps. In the first step, pressure drops at different flow rates for fan (pump)-heat sink model are simulated to construct the system operation curve. The operation curve is then super imposed on the fan (pump) performance curve to identify system operation points which are intersection points between the performance curves and operation curve.

It is thus possible to extract the volumetric flow relations as a function of input voltage to the fan (pump). In the second step, the volumetric flow conditions which represent the operation conditions of the system at variable voltages are then used as an input conditions to 3D fluid flow analysis to extract the average convection heat transfer coefficient ( $h_{\text {av }}$ ) for the particular fan (pump)-heat sink model. The values of $h_{a v}$ as a function of input voltage to the fan (pump) are used 
a convection boundary conditions for the subsequent model. For an input voltage or power less than starting voltage $\left(V_{c r}\right)$ or starting power $\left(P_{c r}\right)$ of the fan (pump), natural convection conditions apply.

Submodel: conjugate heat transfer (SHT) contains the components of the selfcooling device except the fluid domain. SHT has surface boundary conditions that are obtained from SFF. The heat generation rate the device is also an input parameter to the model. In SHT, the governing equations of conservation of energy are applied to calculate the temperature distribution in the whole device setup. These include heat source device, heat spreaders, TEG modules and parts of the heat sink.

There are also planar and volumetric heat generation rates inside the TEG module which are given by Peltier, Joule and Thomson heat effects. The heat generation values are the function of the current flow inside the TEG modules. Thus, it is also necessary to have submodel: electrical circuit (SEC) which calculates the current, voltage and power from TEG modules. SEC basically contains 1D equations which relate current and voltage as a function of temperature difference across the TEG modules.

Thus, we have a loop by which SHT supplies temperature values to SEC which in turn furnishes heating rates back to SHT. SEC also supplies the voltage value 
at each iteration and time step so that the appropriate boundary condition value is applied in SHT.

\subsection{Finite element model}

\subsubsection{Physical configuration of model}

The baseline simulation model is based on the configuration as used by the experimental work by Martinez et al[78]. This configuration is a single stage series type arrangement. It consists of an aluminum plate which is heated by two square heating elements placed inside the plate. TEG modules are wedged between the heated plate and cooling system. The cooling system consists of an aluminum extender and parallel-plate fin heat sink. A fan is placed on top of the finned heat sink to provide cooling.

\subsubsection{Equation based modeling in FEM model}

The numerical simulation is carried out using the commercial finite element method (FEM) based software package (COMSOL) [79]. Due to symmetry, 2D coupled simulation is made on one half part of the model. A weak form mathematical equation is used to represent the thermoelectric effect. It is an integral form equivalent to the original partial differential equation (PDE) and is derived by multiplying the PDE with a test function and integrating over the domain. A separate 3D full domain laminar flow simulation on the heat sink and 
fan is made to extract convection heat transfer coefficient $\bar{h}$ values at the fan operating conditions. The rest of the outer surface, which has a fixed convection heat transfer of $\bar{h}=8.8 \mathrm{~W} / \mathrm{m} 2 . \mathrm{K}$, is also derived from full domain simulation free convection between the surfaces and the surrounding air. The data is also validated by the experimental data.

Grid independence study was carried out to verify the grid independence of the solution obtained to assure the validity and accuracy of the results. A grid system 9662 element which is termed as normal grid as compared with finer grid of 11695 elements and coarser grid of 3906 elements. The relative error between the different grid sizes for local temperature and electric potential is found to be less than $0.01 \%$ which shows that the solution is grid size independent. The time taken for a single run on Intel $\AA^{\mathrm{X}} \mathrm{Xeon} \otimes \mathrm{CPU} @ 2.40 \mathrm{GHz}$ is recorded to be $3 \mathrm{~s}, 4$ $\mathrm{s}$ and $6 \mathrm{~s}$ for the fine grid, normal grid and coarse grid respectively.

\subsubsection{Validation of numerical model}

Figure 4-2 shows the comparison between present numerical simulation model and the experimental data by Martinez et al[78]. The dashed lines represent numerical simulation results while the experimental results are denoted by a triangle and square markers. It could be seen that the simulated results are in good agreement with an average error of $3 \%$ for $\Delta \mathrm{T}_{\mathrm{g} \text {-amb }}$ and around $6 \%$ for $\Delta T_{g-a m b}$. Thus, the $2 \mathrm{D}$ model is very effective in simulating the multiphysics 
problem with good accuracy and faster. The efficiency of the TEG, ПTEG from the numerical model is calculated to be $5.17 \%$ for the heating power input of $220 \mathrm{~W}$, as compared to the manufacturer's supplied data of the efficiency for Kyrotherm TGM-287-1.0-1.5 model of 5.0\% [at $\left.\Delta \mathrm{T}=170{ }^{\circ} \mathrm{C}\right]$.

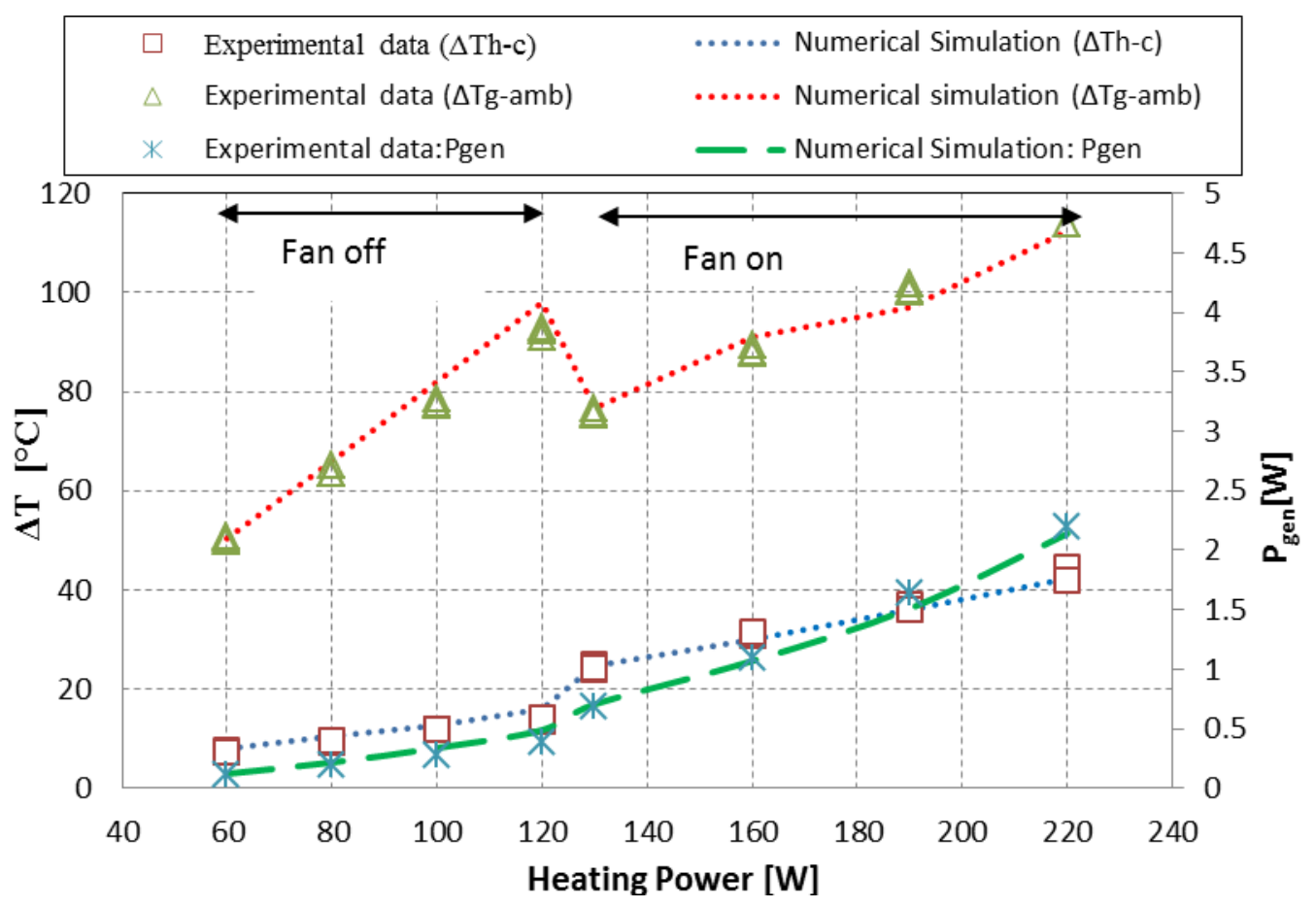

Figure 4-2 Comparison between numerical simulation model and experimental data

This shows that the numerical model is able to represent the efficiency of the physical model with almost $97 \%$ accuracy. 


\subsubsection{Parametric study using FEM model}

The design of the heat sink geometry with respect to self-cooling is one of the important parameters that could affect the performance of the entire system. For the baseline model (BM), the effects of fin density, i.e. the number of fins per unit length of fin $\left(\mathrm{N}_{\text {fin }}\right)$, and the effects of the height of fins $\left(\mathrm{H}_{\mathrm{fin}}\right)$, on the maximum temperature of the heated plate and power generated by the TEGs are studied.

\subsubsection{Effect of fin density ( $\left.N_{\text {fin }}\right)$}

Figure 4-3 shows the performance of the fan coupled with TEG system for different values of fin densities (The subscripts 1.5, 2.1, 2.6 represent $N_{\text {fin }}$ equal to 1.5 fins $/ \mathrm{cm}, 2.1$ fins $/ \mathrm{cm}$ and 2.6 fins $/ \mathrm{cm}$ respectively. The subscripts $4 \mathrm{~V}, 8 \mathrm{~V}$, $12 \mathrm{~V}$ represent the fan performance curve when the supplied voltage is equal to $4 \mathrm{~V}, 8 \mathrm{~V}$ and $12 \mathrm{~V}$ respectively). One unique feature of self-cooling system is the variation with temperature of the fan input power and voltage from TEG. Thus, a plot of fan performance curve at various voltages is made. This is adapted from manufacture data for the fan SUNON KDE1212PTB1 which is only given for 12 V. The fan was applied to derive the performance data at other voltages. The simulated fan operating performance is superimposed on the data to find the fan operating conditions. This also enables to derive the power consumption for the fan assuming an average fan efficiency of $25 \%$. This graph would be important to understand the ranges of geometrical and heating power combination which 
enables self-cooling to be effective. The fan performance data is also derived for different fin density. As $\mathrm{N}_{\text {fin }}$ is varied from 1.5 fins $/ \mathrm{cm}$ to 2.6 fins $/ \mathrm{cm}$, the fan performance data shifts to the left signifying more pressure loss per unit volume flow rate. This is due to the extra resistance to the air flow due to an increased number of fins per area. The power consumed by the fan also increases with increased fan density to counter the extra pressure losses. The power consumed by the fan increases by almost $136 \%$ at $12 \mathrm{~V}$ and $85 \%$ when operated at $8 \mathrm{~V}$.

However, the extra number of fins helps with heat dissipation reducing the increases in the maximum temperature of the heated plate, $\mathrm{Tg}$, as shown in Figure 4-4. The figure demonstrates that the value of $\mathrm{Tg}$ decreases by almost $4.34 \%$ when the Nfin increased from 1.5 fins $/ \mathrm{cm}$ to 2.6 fins $/ \mathrm{cm}$ at the heating power of $220 \mathrm{~W}$. This is due to a decrease in thermal resistance from $0.53{ }^{\circ} \mathrm{C} / \mathrm{W}$ to close to $0.45{ }^{\circ} \mathrm{C} / \mathrm{W}$. The highest increase in $\mathrm{Tg}$ is observed at the lower heat input of $130 \mathrm{~W}$ with an almost $5.8 \%$ increase as Nfin is increased to $2.6 \mathrm{fins} / \mathrm{cm}$. However, the temperature difference, $\Delta T h-c$ and the power generated by the TEG Pgen changed only slightly for an increases in fin density at a particular heating power. This is an interesting observation that for such type of arrangement where the heat sink is coupled with the heat source via the TEG. 


\subsubsection{Effect of fin height $\left(H_{\text {fin }}\right)$}

The height of the fin is varied from $23 \mathrm{~mm}$ to $50 \mathrm{~mm}$ for the same fin density of 32 fins/cm. It can be observed from Figure 4-5 (The subscripts 23,32,50 represent $\mathrm{H}_{\text {fin }}$ equal to $23 \mathrm{~mm}, 32 \mathrm{~mm}$ and $50 \mathrm{~mm}$ respectively) that the height of fin inversely affects the pressure drop per volume flow rate. As the $\mathrm{H}_{\text {fin }}$ is increased, the fan consumes less power. The power consumed by the fan decreased by $50 \%$ when the $\mathrm{H}_{\text {fin }}$ is doubled at $12 \mathrm{~V}$. The increase in pressure drop is less pronounced at the critical voltage of $4 \mathrm{~V}$. This is due to the low volume rate and pressure drop at low voltage operation.

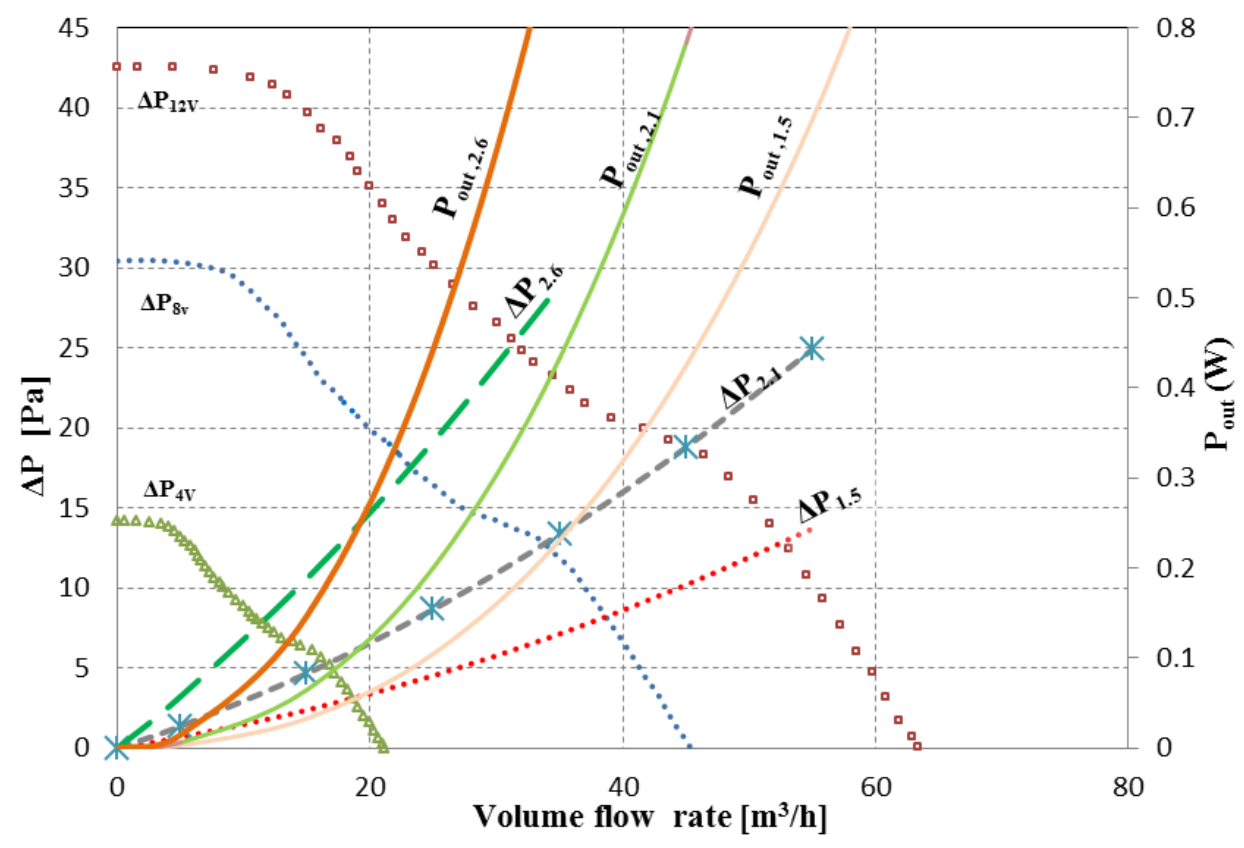

Figure 4-3 . Cooling fan performance and power consumed by the fan as a function of volume rate of air in the fan for different fin densities. 


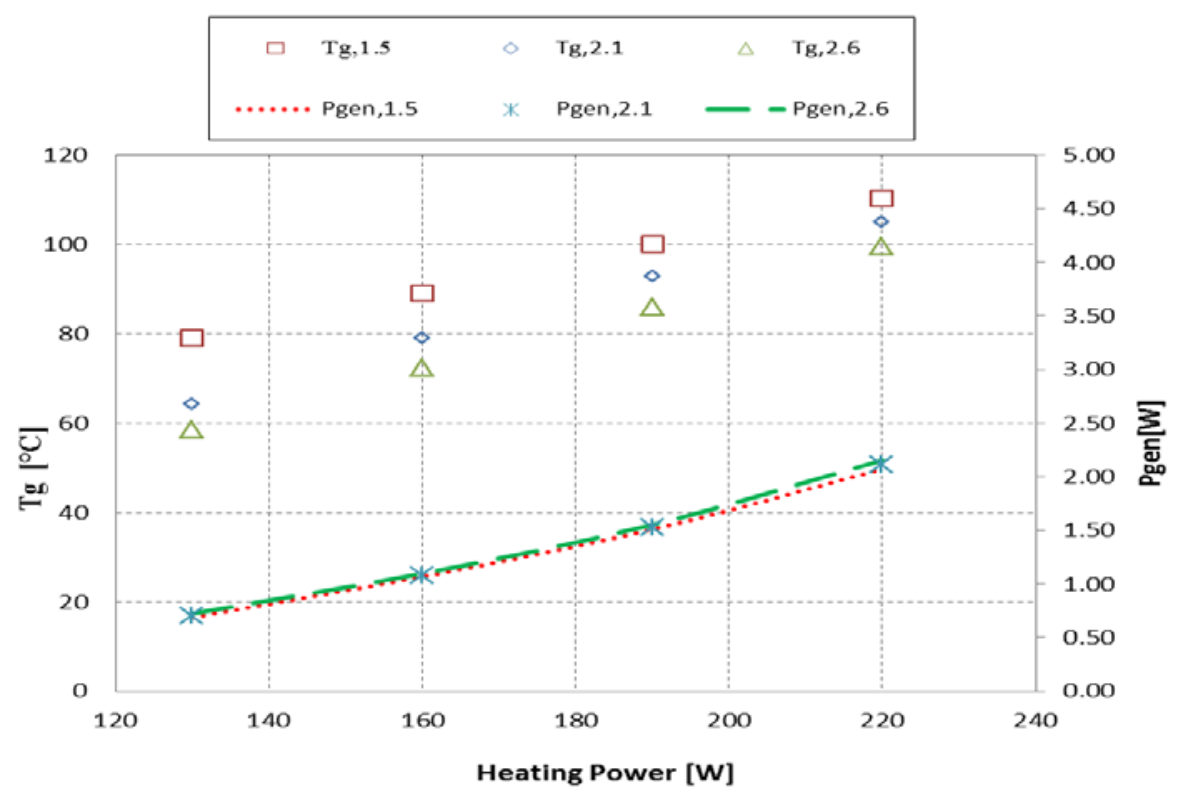

Figure 4-4 Maximum temperature of the heated plate, $\mathrm{Tg}$ and power produced by the TEG, Pgen as a function of heating power supplied to the heater.

Figure 4-6 indicated that the maximum temperature at the heated plate decreases when the height of the fin increases for the same heating power due to an increase in the heat dissipation area. The temperature decreased from almost $65{ }^{\circ} \mathrm{C}$ to a little over $50{ }^{\circ} \mathrm{C}$ with $27 \mathrm{~mm}$ increase in height from $23 \mathrm{~mm}$. At the heating power of $220 \mathrm{~W}, \mathrm{Tg}$ decreases by about $8 \%$ and $22 \%$ when the Hfin is increased from $23 \mathrm{~mm}$ to $32 \mathrm{~mm}$ and $50 \mathrm{~mm}$ respectively. However, the temperature difference along the TEG and power produced by TEG showed little variation as the $\mathrm{Hfin}$ is varied. However, the additional height has to be considered in terms of the space availability for the dissipater 


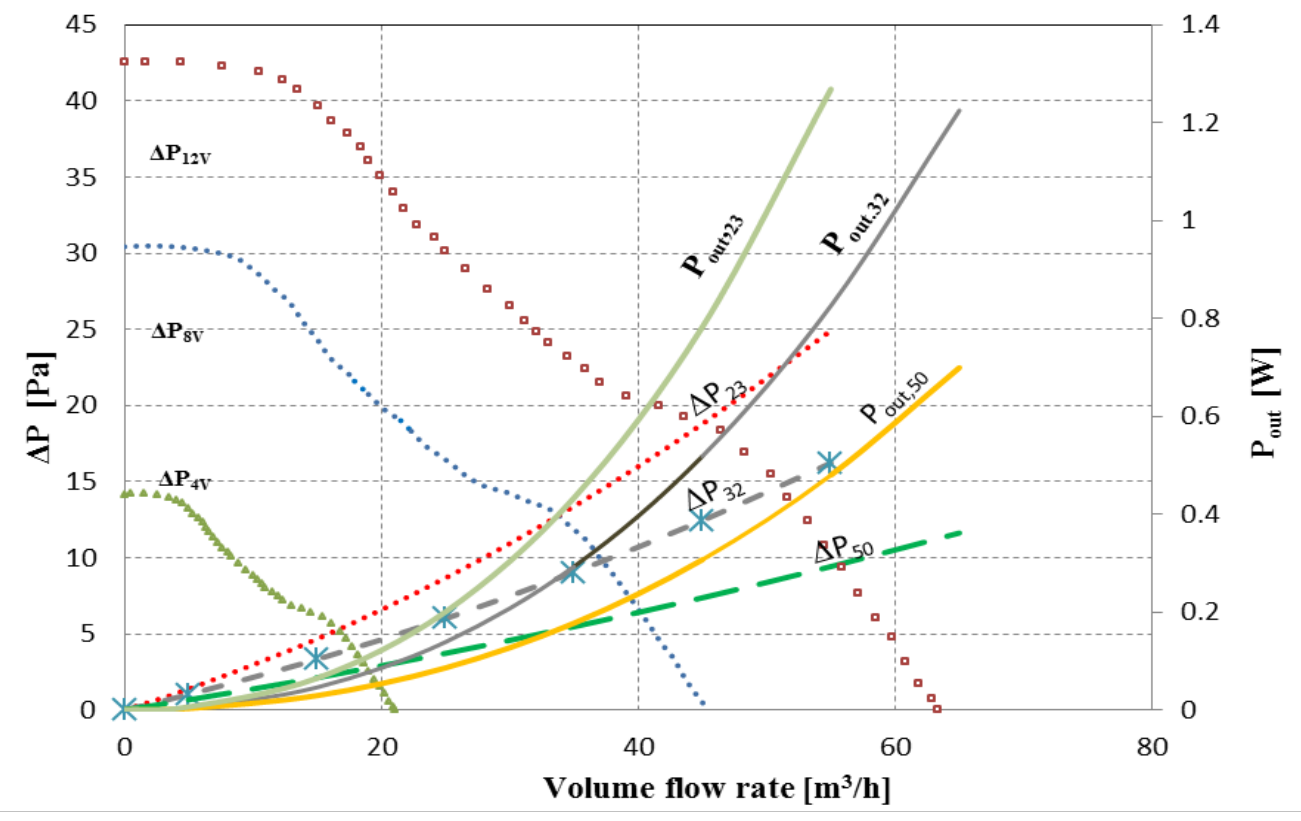

Figure 4-5 Cooling fan performance and power consumed by the fan as a function of volume rate of air in the fan for different fin densities.

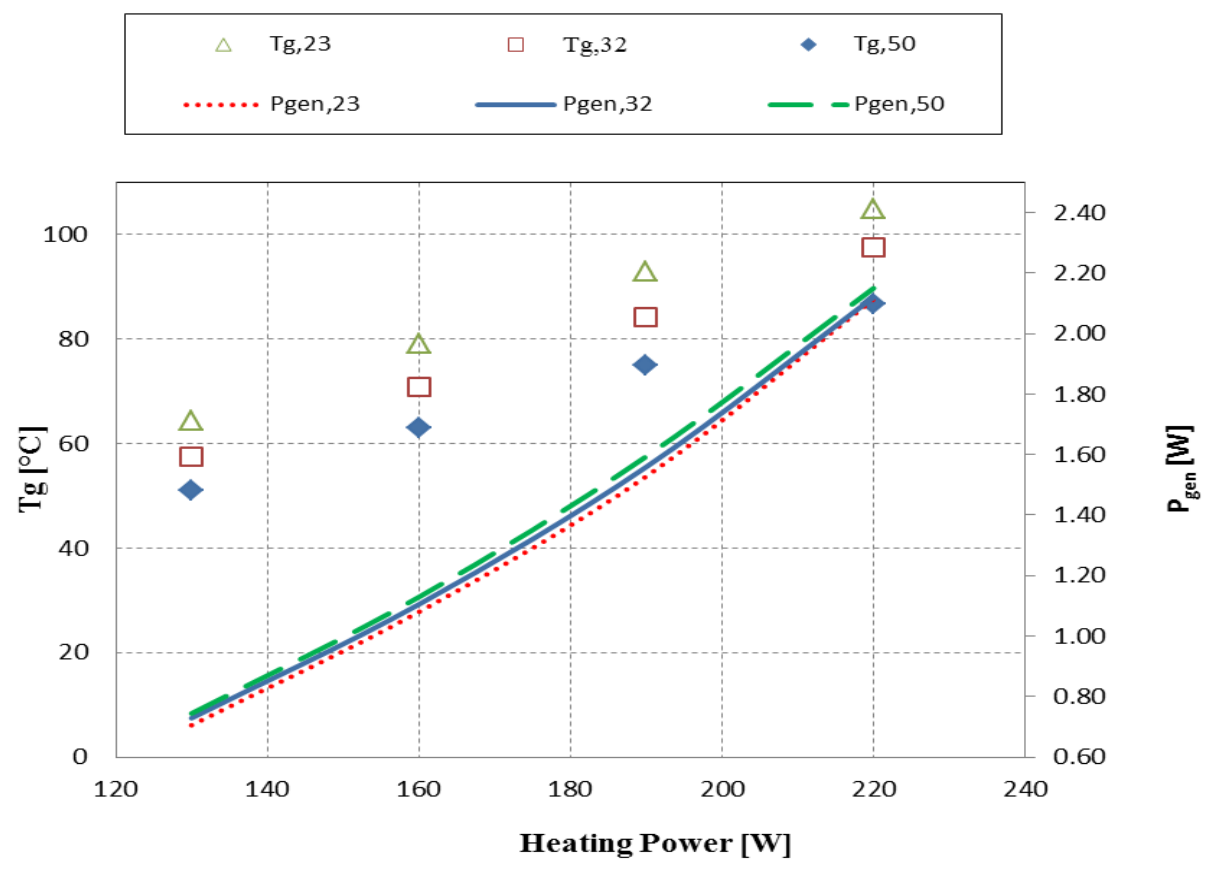

Figure 4-6 Maximum temperature of the heated plate and power produced by the TEG, $P_{g e n}$, as a function of heating power supplied to the heater. 


\subsection{Finite volume model}

The numerical methodology has been implemented using commercial CFD software ANSYS 14 [80] utilizing in-built fluid, heat transfer and electric current modules as well as user defined functions.

\subsubsection{User defined functions based modeling in FVM model}

In this section, a finite volume (FVM) based commercial CFD software (Fluent 14) [80] is used to implement the computational strategy. In Fluent, inbuilt CFD capabilities are integrated with User Defined Functions (UDF) written in ANSI-C. Two computational domains namely domain A and B are constructed.

Domain A represents SFF and domain B has both SHT and SEC integrated into the domain. For a comparison with experimental data, domain $A$ is used to identify the operational points of the fan-heat sink assembly for a given fan used in experiments. It supplies a surface boundary condition to domain B using an interpolating user defined function (UDF). Domain A contains the air domain and the heat sink only. The inlet velocity (volume flow rate) is varied as an input condition. The outlet condition from the domain is zero gage pressure boundary conditions. The governing equations for three dimensional, forced, steady state and incompressible fluid flow are solved. 
Domain B is comprised of the heat sink, extender, array of TEG modules, spreader, heat source heater and the plate.Fully coupled multiphysics modeling technique is used to model fluid flow, heat transfer and electrical subsystems in domain A and B. The governing equations for three dimensional, forced, transient incompressible fluid flow and heat transfer in the device, heat spreader, TEG, cold-side extender, dissipater and air subdomain are solved.

The general equations for continuity, momentum and energy are given as follows.

Continuity equation:

$$
\frac{\partial u}{\partial x}+\frac{\partial v}{\partial y}+\frac{\partial w}{\partial z}=0
$$

where $u, v$ and $w$ are the fluid velocity components in $x, y$ and $z$ directions respectively.

Momentum equations in $x, y$ and $z$ coordinate:

$$
\rho_{f}\left(\frac{\partial u}{\partial t}+u \frac{\partial u}{\partial x}+v \frac{\partial u}{\partial y}+w \frac{\partial u}{\partial z}\right)=-\frac{\partial P}{\partial x}+\mu_{f} \nabla^{2} u
$$




$$
\begin{aligned}
& \rho_{f}\left(\frac{\partial v}{\partial t}+u \frac{\partial v}{\partial x}+v \frac{\partial v}{\partial y}+w \frac{\partial v}{\partial z}\right)=-\frac{\partial P}{\partial y}+\mu_{f} \nabla^{2} v \\
& \rho_{f}\left(\frac{\partial w}{\partial t}+u \frac{\partial w}{\partial x}+v \frac{\partial w}{\partial y}+w \frac{\partial w}{\partial z}\right)=-\frac{\partial P}{\partial z}+\mu_{f} \nabla^{2} w
\end{aligned}
$$

where $P$ is the pressure field.

Energy equation

$$
\frac{\partial T}{\partial t}+u \frac{\partial T}{\partial x}+v \frac{\partial T}{\partial y}+w \frac{\partial T}{\partial z}=\frac{k_{f}}{\rho_{f} C_{p}} \nabla^{2} T
$$

The computational domains and boundary condition are depicted in Figure 4-7. For the numerical simulation, a uniform volumetric heat generation $\left(\dot{Q}_{i n}\right)$ is assumed in a heater. All surfaces of the heated plate except the side in contact with the spreader are assumed to be insulated. The ambient temperature is taken as $298 \mathrm{~K}$. Adiabatic boundary conditions were applied to TEG walls exposed to the environment.

A thermoelectric module consists of number of TEG modules connected in series by copper solder and contained between thermally conductive and electrically insulated ceramic plates. The simulation of numerous P-N pairs inside each module in an array would result in high computational complexity in terms of grid 
size as well as computational time [81]. Thus, the TEG module is modeled as TEG cuboid consisting of three parts as shown in Figure 4-8. The first part represents $\mathrm{P}$ and $\mathrm{N}$ semiconductor pellets with an air gap. It is modeled as a combined pellet-air gap cuboid by aggregating the properties of the $\mathrm{P}$ and $\mathrm{N}$ legs and the air gap in between using the weighted mean method. The thermal properties of aluminum, copper and ceramic at an average temperature of $350 \mathrm{~K}$ is shown in Table 1 [82].

Table 4-1 Physical properties of aluminum, copper and ceramic

\begin{tabular}{llllll}
\hline Physical properties Symbol Units Aluminum Copper & Ceramic
\end{tabular}

$\begin{array}{llllll}\text { Thermal conductivity } & \mathrm{k} & \mathrm{W} /(\mathrm{m} . \mathrm{K}) & 237 & 398 & 25.12 \\ & & & & & \\ \text { Electrical resistivity } & \mathrm{R} & \Omega \mathrm{m} & 2.82 \times 10^{-8} & 1.67 \times 10^{-8} & 5.03 \times 10^{10}\end{array}$

The thermal properties of Seebeck coefficient, thermal conductivity and for the $p$ $\mathrm{n}$ elements are assumed to isotropic and adapted from [69]

$$
\alpha_{p, n}=-99 \times 10^{-7} T_{m, a v}^{2}+9.30 \times 10^{-7} T_{m, a v}+220 \times 10^{-7}(V / K) \quad 4.4 .6
$$




$$
\begin{gathered}
k_{p, n}=4.23 \times 10^{-5} T_{m, a v}^{2}-2.77 \times 10^{2} T_{m, a v}+62.6 \times 10^{3}(\mathrm{~W} /(\mathrm{mK})) \quad 4.4 .7 \\
R_{p, n}=63 \times 10^{-12} T_{m, a v}^{2}+1.6 \times 10^{2} T_{m, a v}+5.11 \times 10^{3}(\Omega m) \quad 4.4 .8
\end{gathered}
$$

The second part is the copper conductors on both sides of the pellet-air gap cuboid. The copper conductors are also aggregated into a homogenous conductor cuboid by similar method used for the semi-conductor pellets. The third part is the cold and hot side ceramic plates. At the interface between the cold side ceramic plate and the base of the heat sink and hot side of ceramic plate and spreader, there exits an interfacial contact thermal resistance.

Thus, the thermal properties of the ceramic plates have been modified to account of the extra contact thermal resistance. The value for the contact thermal resistance has been estimated from experimental data. 


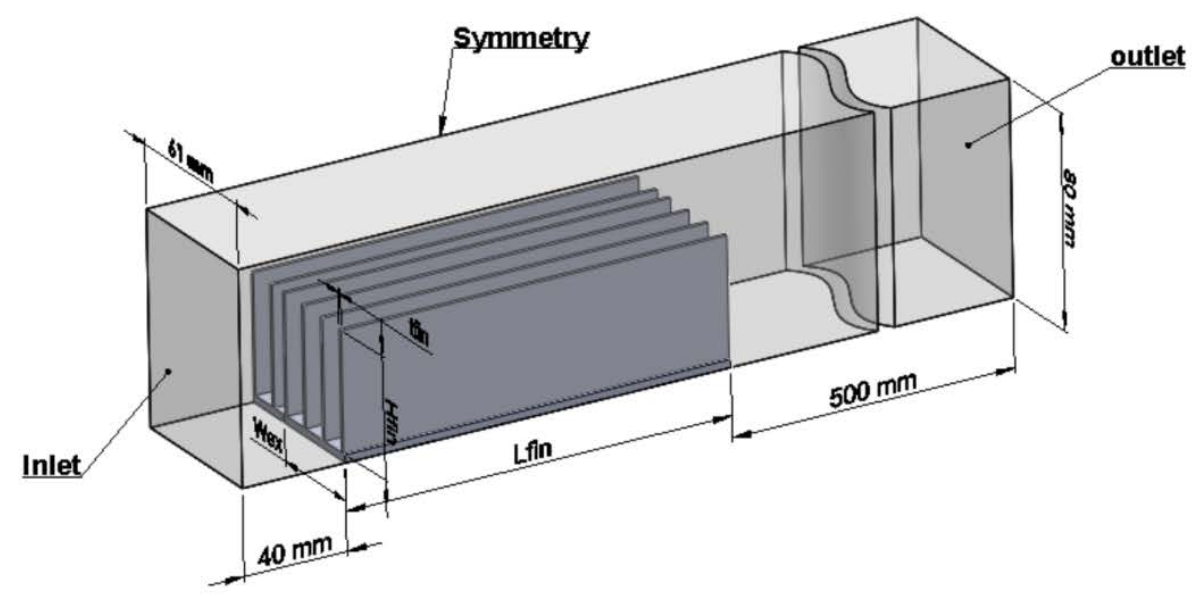

Domain A

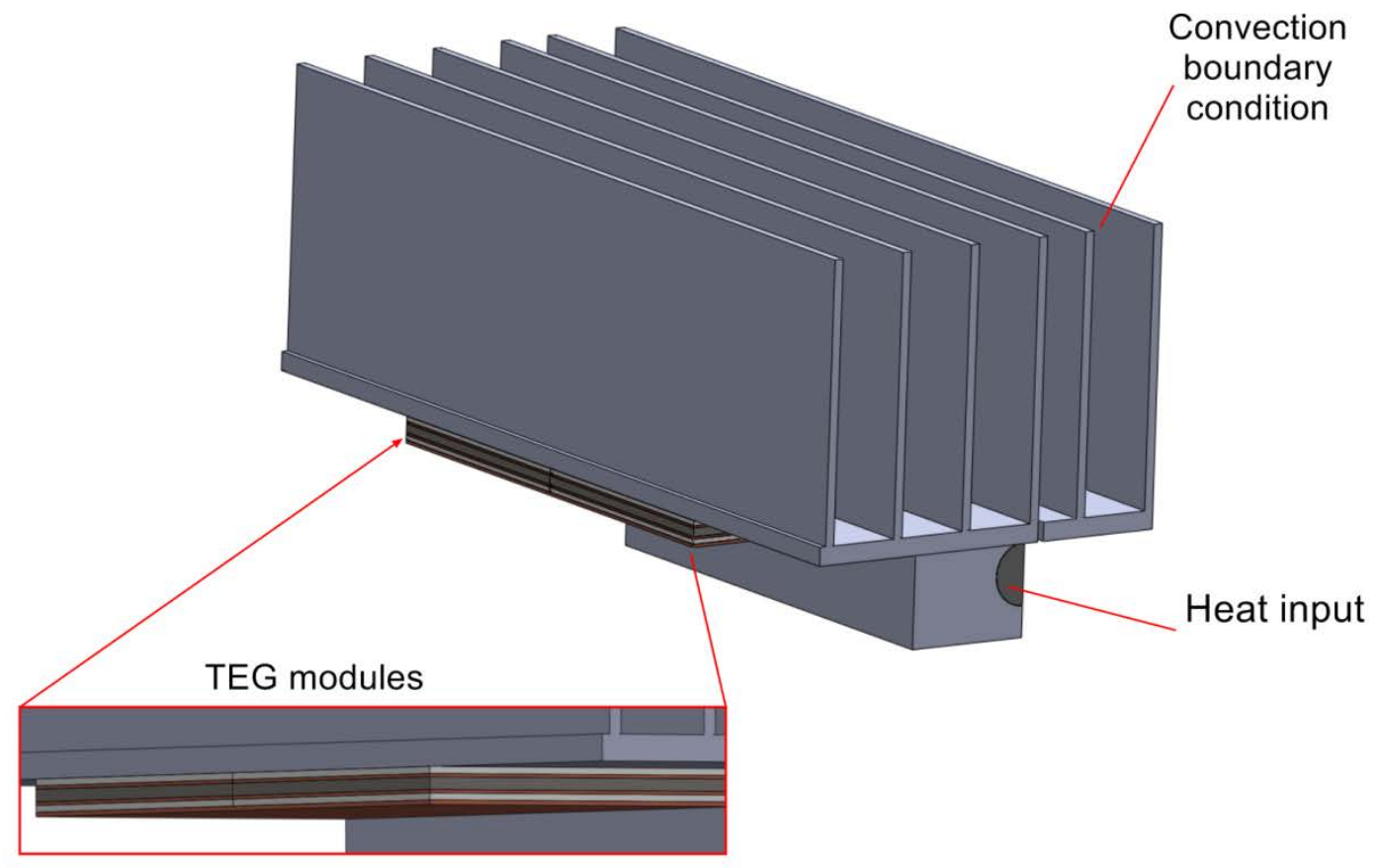

Domain B

Figure 4-7 Computational domain A and B in ANSYS 

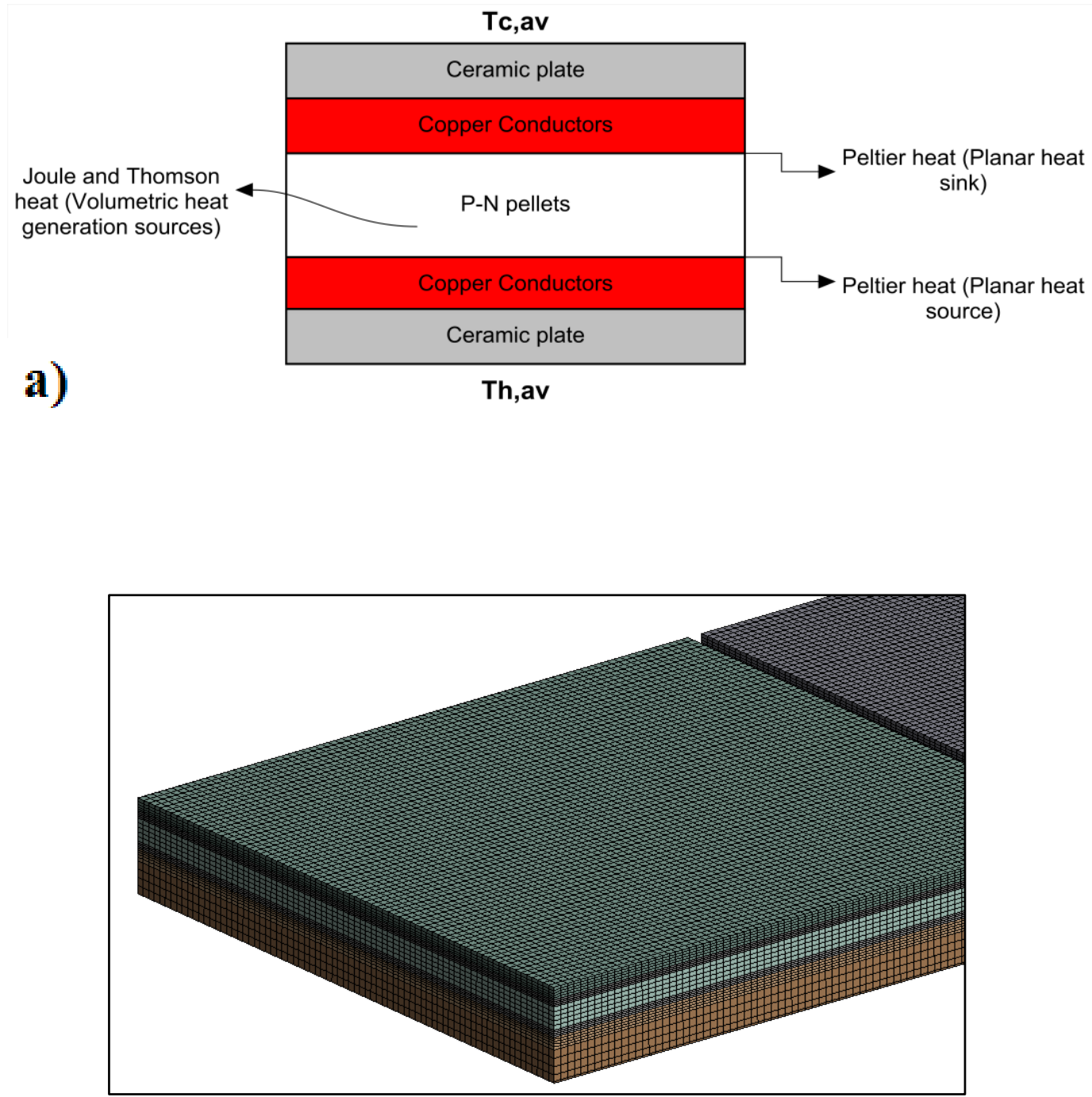

b)

Figure 4-8 a) Schematic diagram of numerical model b) computational grid of TEG module 
For an array of TEG modules connected in series to a load, total voltage at any given instant $t, V_{T}(\mathrm{t})$ and the current in the array, $I(t)$ are given as:

$$
V_{T}(t)=\sum_{j=1}^{n_{a}}\left(\left(T_{h, a v_{-} j}(t)-T_{c, a v_{-} j}(t)\right) \alpha_{M}\left(T_{m, a v}\right)-\left(m R_{e, T E G}\left(T_{m, a v}\right)+R_{e, l d}+R_{e, c}\right) I(t)\right.
$$

$$
I(t)=\frac{\sum_{j=1}^{n_{a}}\left(T_{h, a v_{-} j}(t)-T_{c, a v_{-} j}(t)\right) \alpha_{M}\left(T_{m, a v}\right)}{m R_{e, T E G}\left(T_{m, a v}\right)+R_{e, l d}+R_{e, c}}
$$

where $m$ is the number of modules in an array and $T_{h, a v j}(t)$ and $T_{c, a v \_j}(t)$ are the average hot side and cold side temperature of the jth TEG module at an instant $t$ respectively.

The fan supply power is also related to the fan supply voltage $V_{x}$ by the equation:

$$
P_{x V}=\frac{\left(V_{x}\right)^{2}}{R_{l d}}
$$

For the m number of modules connected in series electrically in an array, the average temperature of hot side and cold side of the jth TEG module could be calculated as: 


$$
T_{a v, j}=\frac{\left(\iint_{t_{0}}^{t_{1}} T(x, y, z, t) d x d y d z d t\right)_{j}}{A\left(t_{1}-t_{0}\right)}
$$

where $\left[\mathrm{t}_{0}, \mathrm{t}_{1}\right]$ is a time interval, $\Omega$ is the spatial derivative for the jth module. $T_{h, a v}$ and $T_{c, a v}$ are calculated at the hot and cold surface of the TEG module respectively.

Peltier heat at the interface of hot side of TEG and copper solder at a jth module in an array of TEG modules is given as:

$$
\left(q^{\prime \prime}(t)\right)_{j, \text { peltier,hot }}=\frac{I(t) T_{h, a v_{-} j}(t) N_{P}\left(\alpha_{P}-\alpha_{N}\right)}{A_{T E G, h}}
$$

where ATEG,h is the hot side area of TEG module

Peltier heat at the interface of cold side of TEG and copper solder is given as:

$$
\left(q^{\prime \prime}(t)\right)_{j, \text { peltier,cold }}=\frac{I(t) T_{c, a v_{-} j}(t) N_{P}\left(\alpha_{P}-\alpha_{N}\right)}{A_{T E G, c}}
$$

where $A_{T E G, c}$ is the cold side area of TEG module. 
The Joule and Thomson heat are modeled as volumetric heat generation in the P-N pellet region and could be written as:

$$
\left(q^{\prime \prime \prime}(t)\right)_{j, \text { joule }}=\frac{I(t)^{2} R_{e l, P N} N_{P}{ }^{2}\left(\alpha_{P}-\alpha_{N}\right)}{A_{T E G} L_{P N}}-\frac{I(t) N_{p} T \nabla \alpha\left(T_{h, a \nu_{-} j}-T_{c, a v_{-} j}\right)}{A_{T E G} L_{P N}}
$$

where first and second term in the equation are the Joule and Thomson heat respectively, LPN is the height of the P-N pellet region and $R_{e l, P N}$ is the electrical resistivity of $\mathrm{P}$ and $\mathrm{N}$ thermoelectric pairs

From the equation it could be seen that, the heat terms are a function of the current flowing inside the module and the current is again dependent on the temperature difference between the TEG module surfaces. Thus, it is important to employ a numerical methodology which iteratively calculates for the current and voltage inside the array. The capability of employing User Defined Functions (UDFs) written in ANSI C programming language in ANSYS Fluent (Appendix B) is employed to include the electrical and heat terms.

\subsubsection{Validation of the numerical model}

Figure 4-9 shows the fan performance data at varying voltage. The system operation curve for the heat sink geometry used in the experiment is superimposed on the fan performance data. The numerically simulated data is 
denoted by a full line while the experimental data is represented by dots. The system operation derived from CFD analysis has good correspondence with experimental data. The intersection between the fan performance data at a given voltage and system operation curve indicates the operation point of the fan at that voltage. Therefore, the graph is useful to simulate heat sink pressure drop for different geometries and analyze how the system operates from the lowest starting voltage to the high rated voltage of the fan. Figure 4-10 illustrates the relationship between average heat transfer coefficient and voltage input to the fan for the fan-heat sink model in Case A and B. It is evident that for the voltage less than $V_{c r}$, the fan does not rotate, and cooling off the device is via natural convection. Once the fan starts rotating, forced convection is the dominant means of heat dissipation from heat sink surfaces.

Figure 4-11 (a) and (b) indicate open circuit voltage and power generated by TEG modules per area of TEG arrays as a function of heat input into a device for case A and B respectively. The circular values indicate the experimental data while the lines show the simulated result. It could be observed that for the predicted results are in good agreement with experimental data (within 5\%). The results show that self-cooling starts when the heat input into the device is at least $60 \mathrm{~W}$ and $90 \mathrm{~W}$ for case $\mathrm{A}$ and $\mathrm{B}$ respectively. 


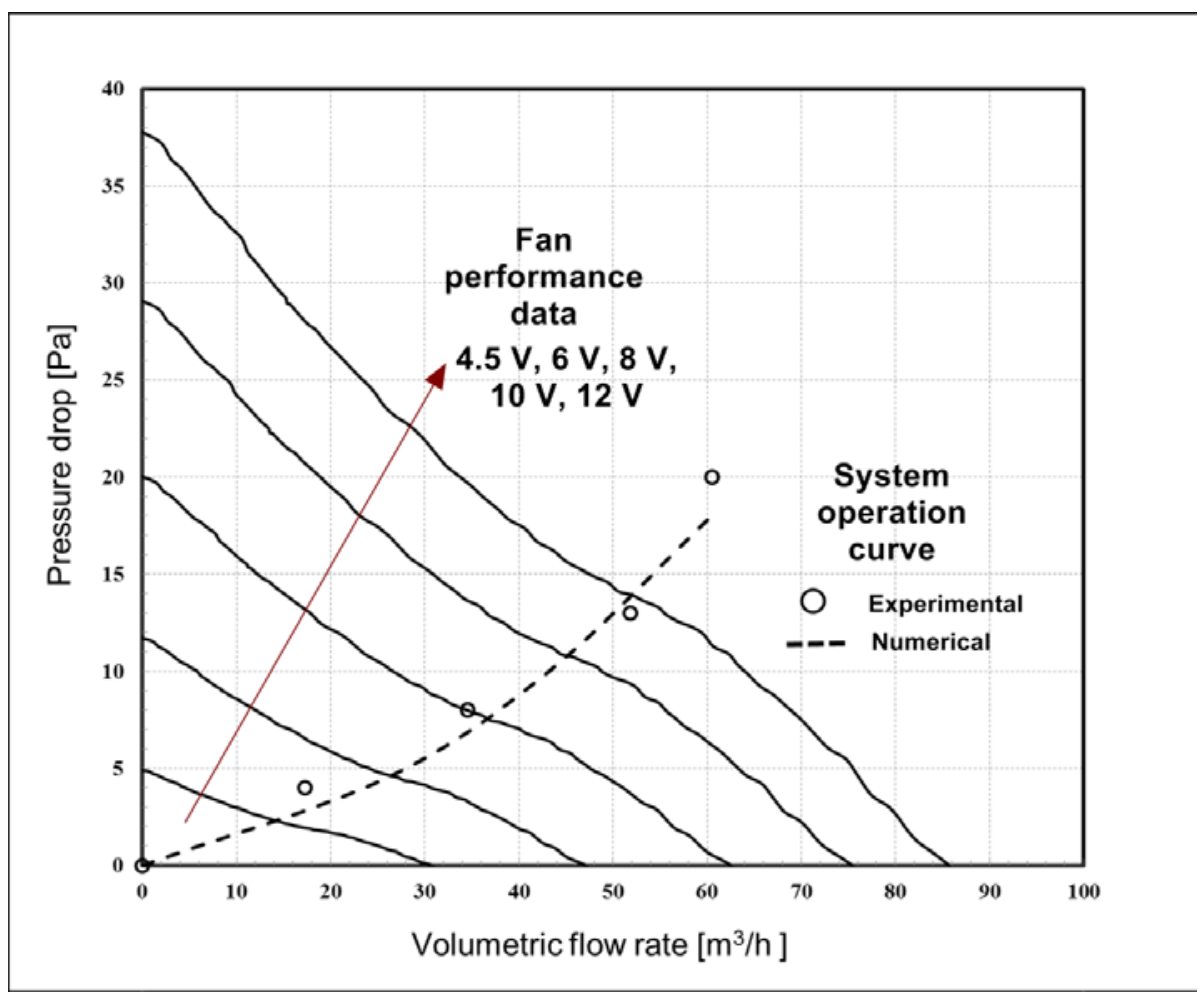

Figure 4-9 Fan performance data and system operation curve for case A and B

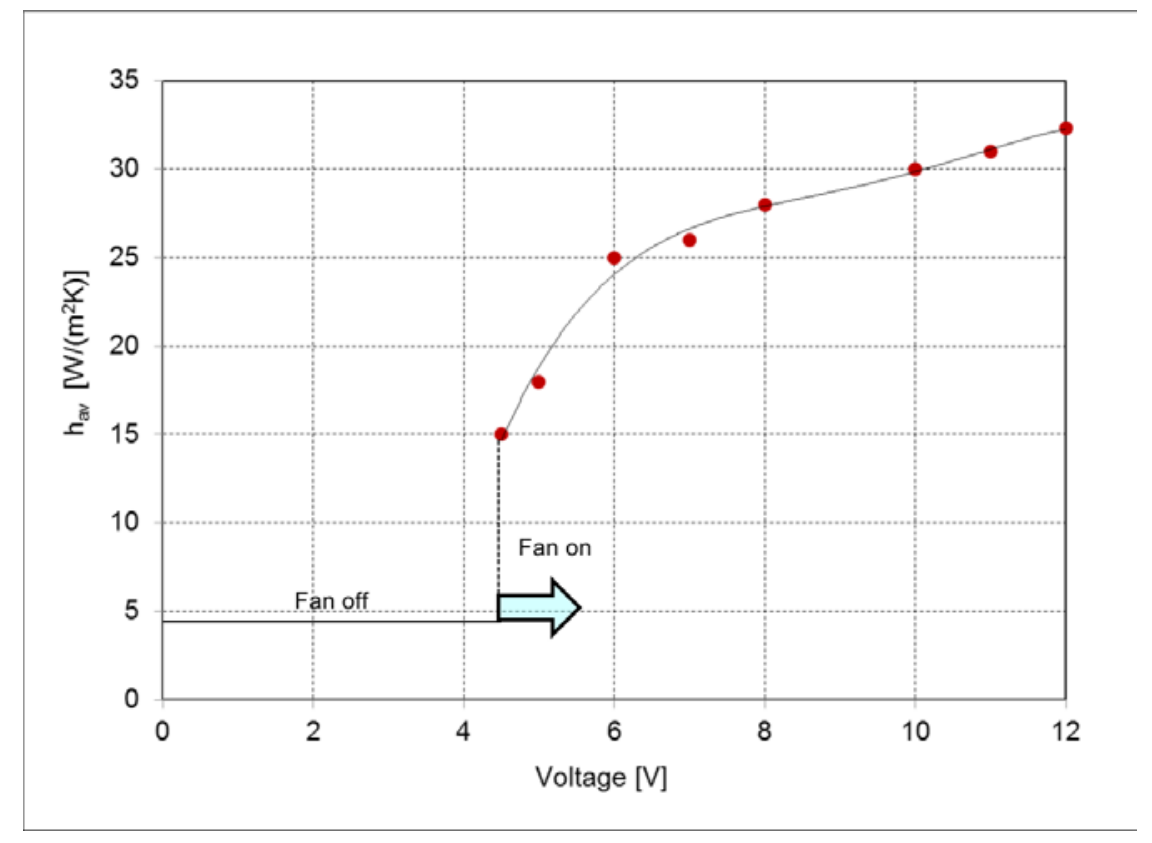

Figure 4-10 Average convection heat transfer coefficient ( $h_{\text {av }}$ ) as a function of voltage 
At the self-cooling zone, the voltage produced by TEG arrays is at least equal to the starting voltage of the fan used in the experiment $\left(\mathrm{V}_{\mathrm{cr}}=4.5 \mathrm{~V}\right)$. when the fan starts to rotate, the internal resistance of the fan suddenly increases from $20 \Omega$ to about $72 \Omega$.

This is marked by the jump in the fan supply voltage and power. Case A produces more total array voltage (38\%) and power per area of TEG array (34\%) than case B. This is, due to the presence of more TEGs in the array and the position of pair of TEGs in direct heat path in case A.

Figure 4-12a and $\mathrm{b}$ show the transient simulation and experimental data of voltage produced by array of modules $\left(\mathrm{V}_{\mathrm{T}}\right)$ and temperature difference between the device and environment $\left(\Delta \mathrm{T}_{\text {de }}\right)$ for $\mathrm{Q}_{\text {in }}=120 \mathrm{~W}$ in Case $\mathrm{B}$ respectively. The numerical simulation has been able to accurately predict both voltage and temperature difference within $5 \%$ error margin. In the experiments, the fan starts to turn at 5 minutes after the heat input is applied at which instant the voltage reaches a value equivalent to $V_{c r}$ as has been precisely simulated.

After the onset of the forced convection from the fan, the rate of change of temperature starts to decrease and the system achieves a steady state after 20 minutes. The temperature doesn't show any appreciable change and the selfcooling has been able to control the temperature effectively. 


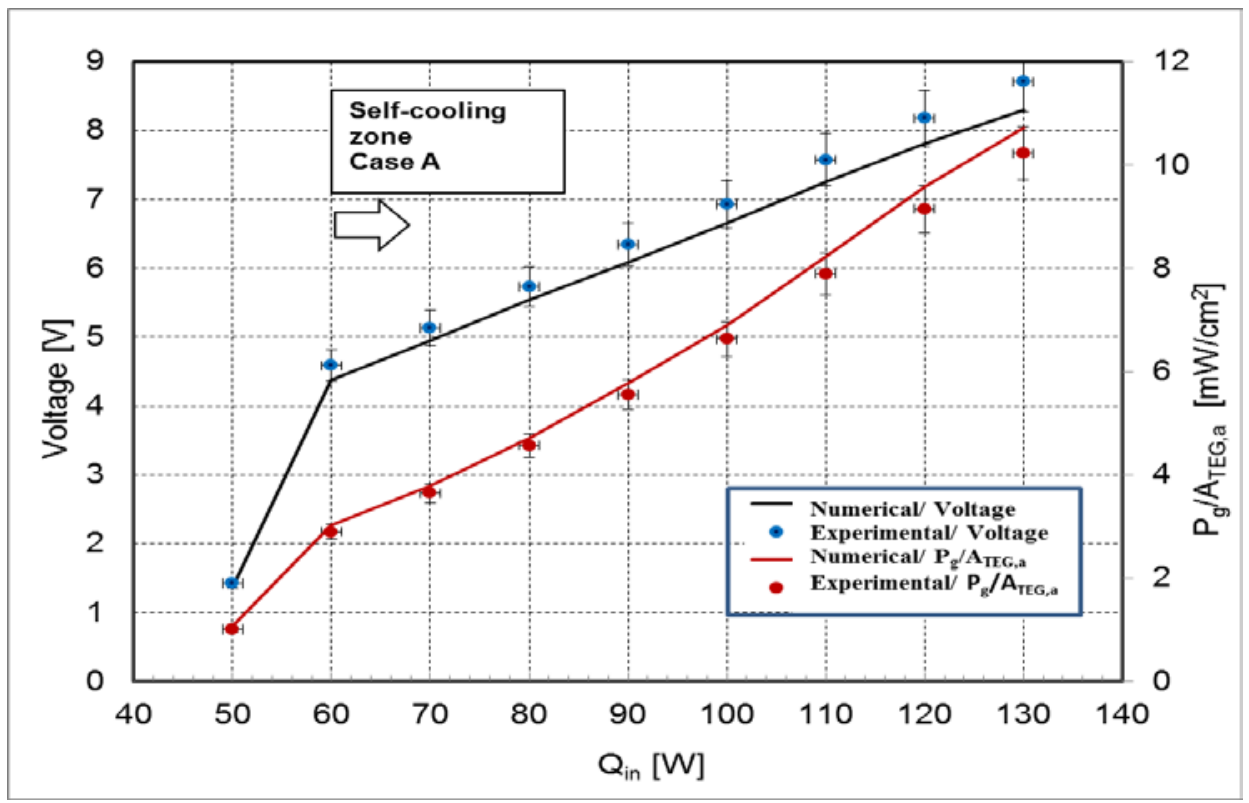

a)

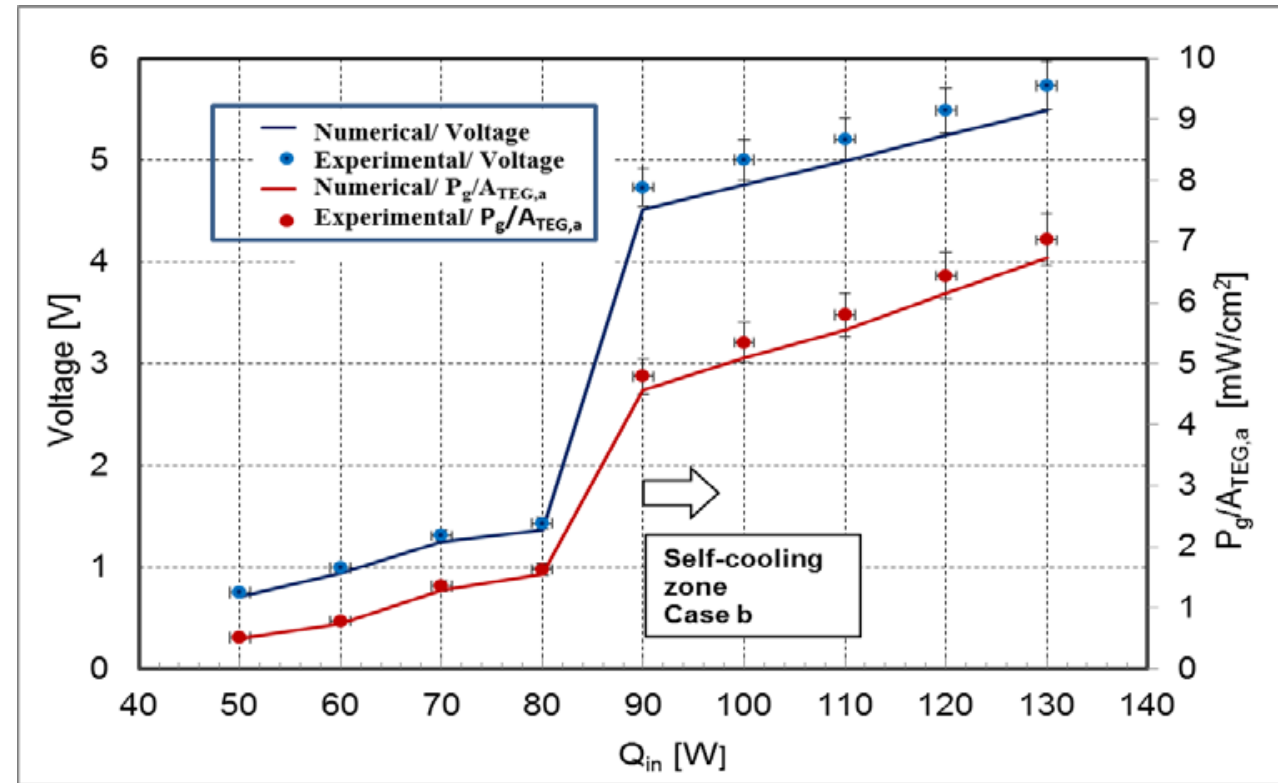

b)

Figure 4-11 a) Total array voltage ( $\mathrm{V}$ ) and Power produced by TEG arrays per area of TEG arrays (PTEG/ATEG,a) for $a$ ) Case $A$ and $b$ ) Case $B$ 


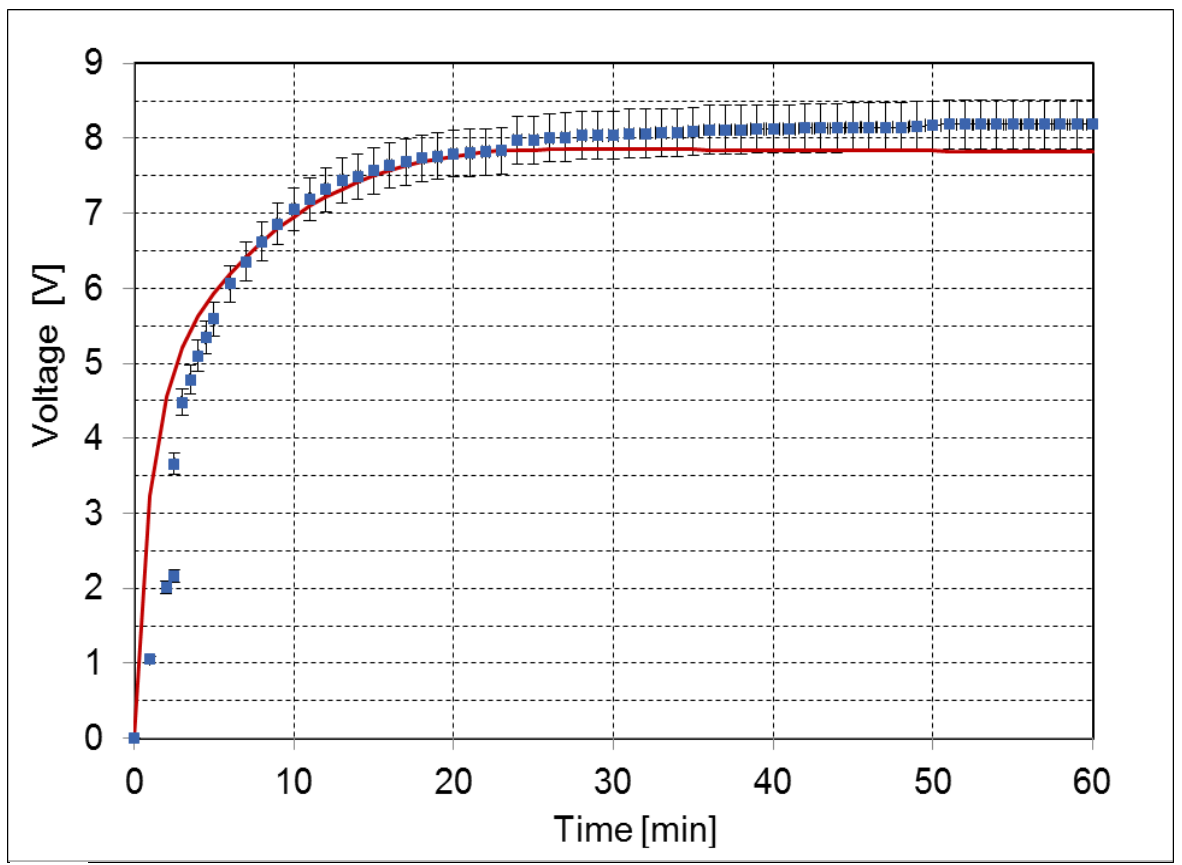

a)

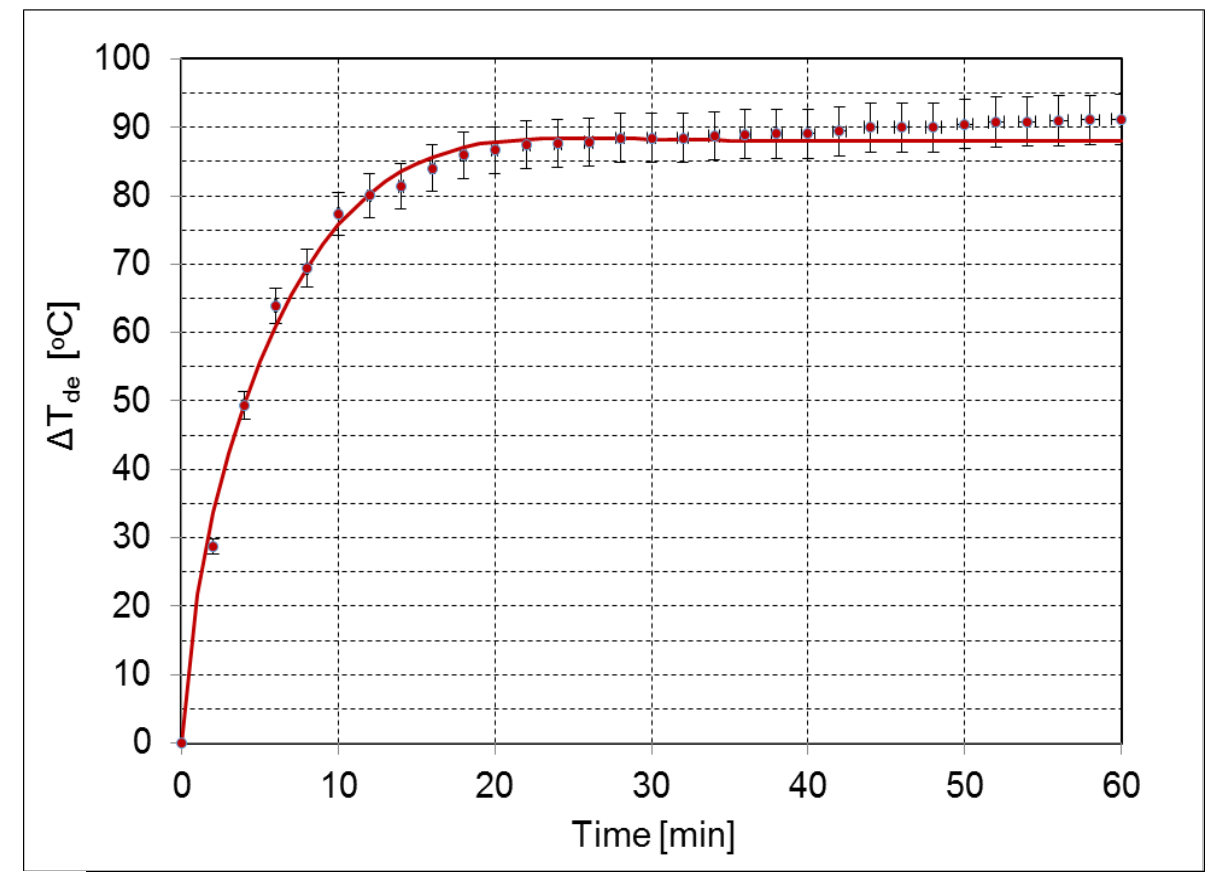

b)

Figure 4-12 Transient simulation of a) voltage supplied to fan b) temperature difference between device and ambient temperature $(\Delta T d e)$ for Qin $=120 \mathrm{~W}$ in Case A 
Figure 4-13 show the transient simulation and experimental data of voltage produced by array of modules $\left(\mathrm{V}_{\mathrm{T}}\right)$ and temperature difference between the device and environment $\left(\Delta \mathrm{T}_{\mathrm{de}}\right)$ for $\mathrm{Q}_{\mathrm{in}}=120 \mathrm{~W}$ in Case $\mathrm{B}$ respectively. The numerical simulation has been able to accurately predict both voltage and temperature difference within $5 \%$ error margin. In the experiments, the fan starts to turn at 5 minutes after the heat input is applied at which instant the voltage reaches a value equivalent to $V_{c r}$ as has been precisely simulated.

Figure 4-14 present the variation of $\Delta T_{\text {de }}$ (difference between temperature of device and environment) as a function of heat input into the device for case A and $\mathrm{B}$. The numerical result has been able to predict the experimental data to good accuracy (within 5\%).

A sharp drop in temperature is observed at $\mathrm{Q}_{\text {in }}=60 \mathrm{~W}$ and $90 \mathrm{~W}$ for case $\mathrm{A}$ and $B$ respectively due to the onset of self-cooling. In general, although case $B$ produces less total array voltage and power per area of TEG, the temperature of the device has been able to be reduced as compared to case A.This is due the presence of less resistance in direct heat path in case A. The numerical result has been able to capture the phenomenon accurately. 

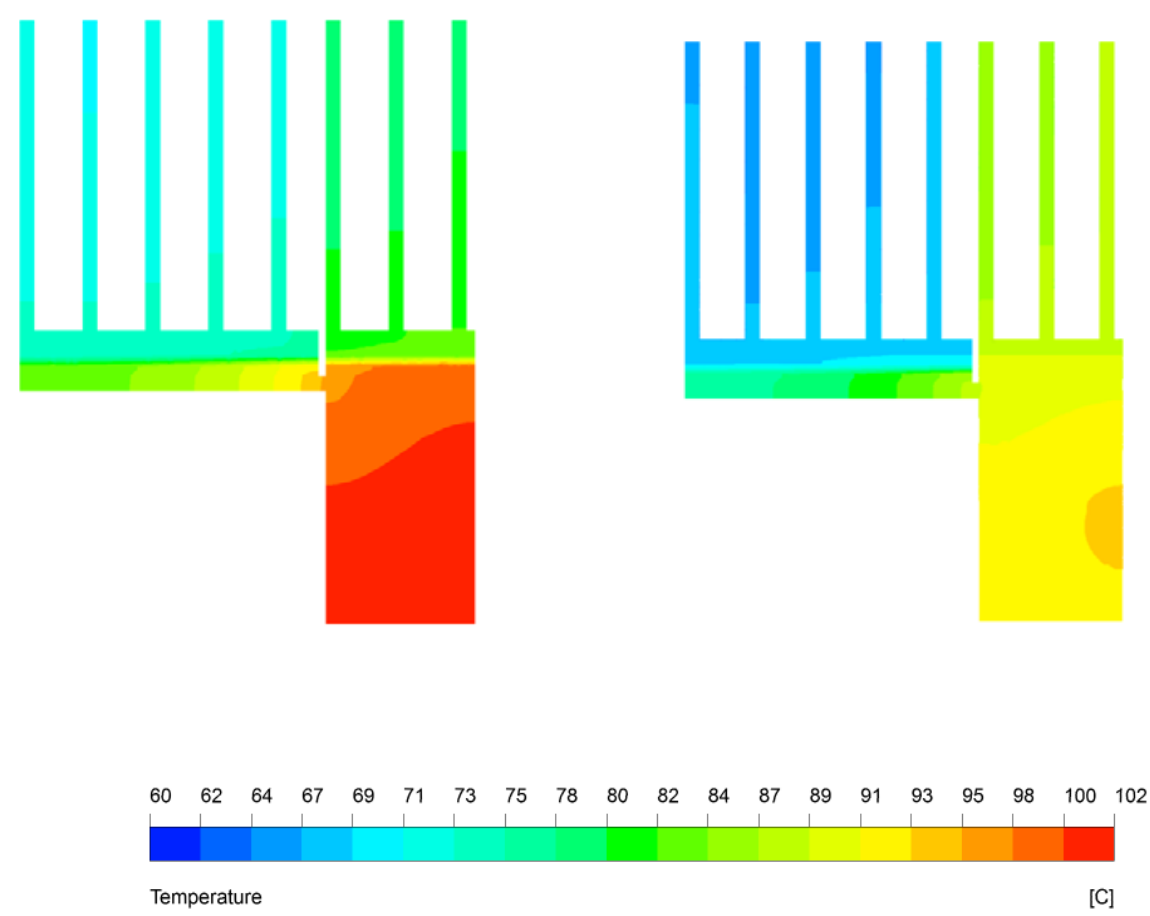

Figure 4-13 Temperature distribution $Q=120 \mathrm{~W}$ a) case $\mathrm{A}$ b) case $\mathrm{B}$ after 60 minutes

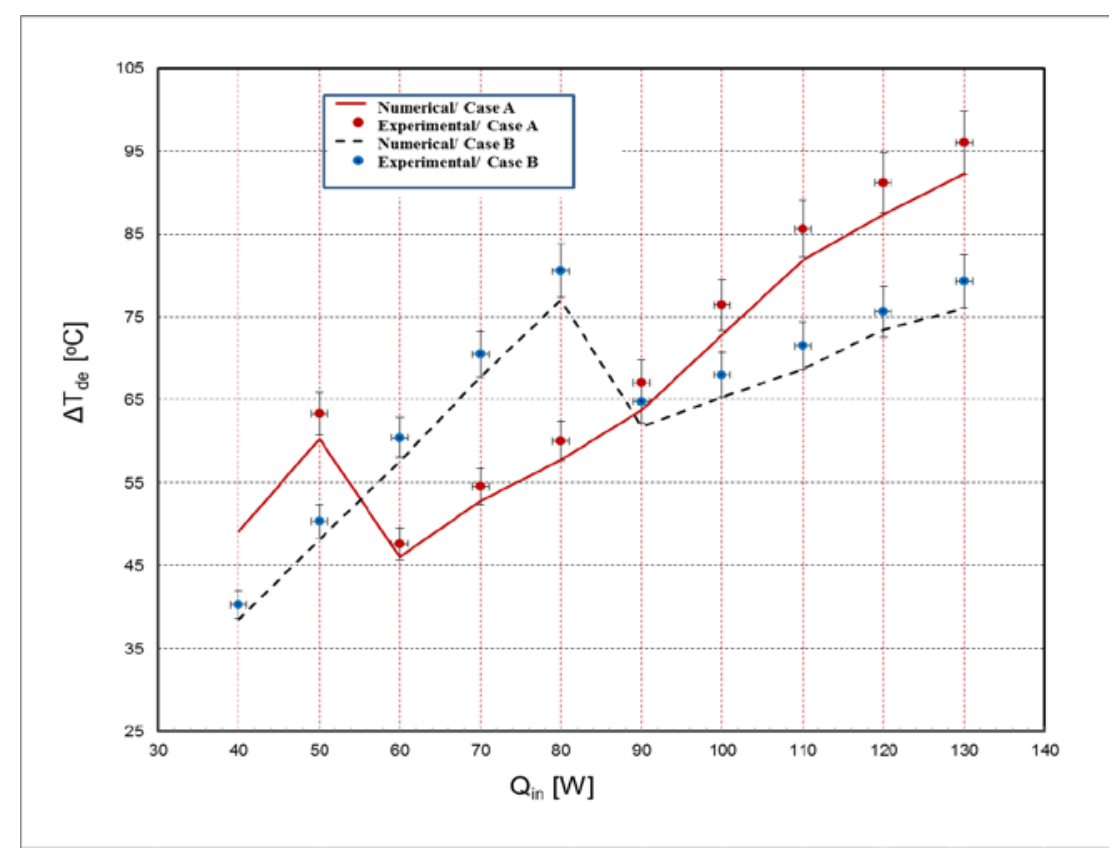

Figure 4-14 Temperature difference between hot and cold side of TEG modules $\left(\Delta T_{d e}\right)$ 


\subsubsection{Parametric study using FVM model}

In the following sections, the FVM model is used for parametric study of the effects of load resistance, fin height and fin density.

\subsubsection{Effect of load resistance}

The electrical resistance of the fan $\left(R_{L}\right)$ affects the amount of power consumed by the fan. The effect of $R_{L}$ on $P\llcorner$ for case $A$ and $B$ is shown in. Figure 4-15 .Each TEG module used in the experiments and simulation has an internal electrical resistance (RTEG) of $10 \Omega$. Thus, for case $A$, the system has maximum $P\llcorner$ at when $R\llcorner$ is equal to $60 \Omega$ which is equivalent to the combined electrical resistance of the six TEGs connected in series. This is due to matched load condition which produces maximum power[69]. It can be inferred that $P\llcorner$ could decrease by as much as $50 \%$ when a fan with $10 \Omega$ is used for heat input of 130 W compared to the maximum point of operation. The fan used in the experiment has an internal resistance of $72 \Omega$ producing a power close to the maximum possible power. For case B, the four TEGs have a cumulative RTEG of $40 \Omega$. Thus, the system produces $10 \%$ less power when connected to the same fan as Case A. It is therefore important to match the electrical resistance of TEG arrays with the fan used in self-cooling applications. 


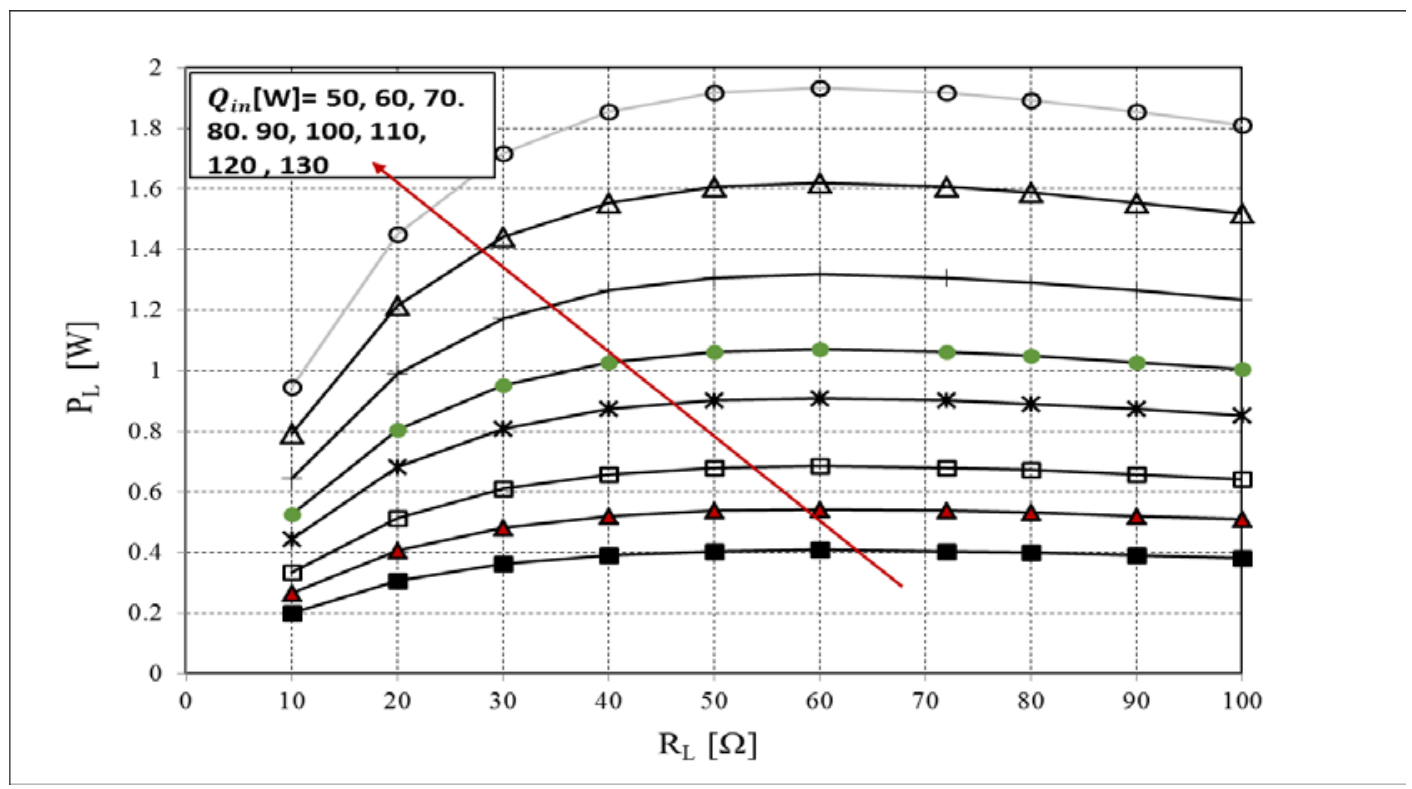

a)

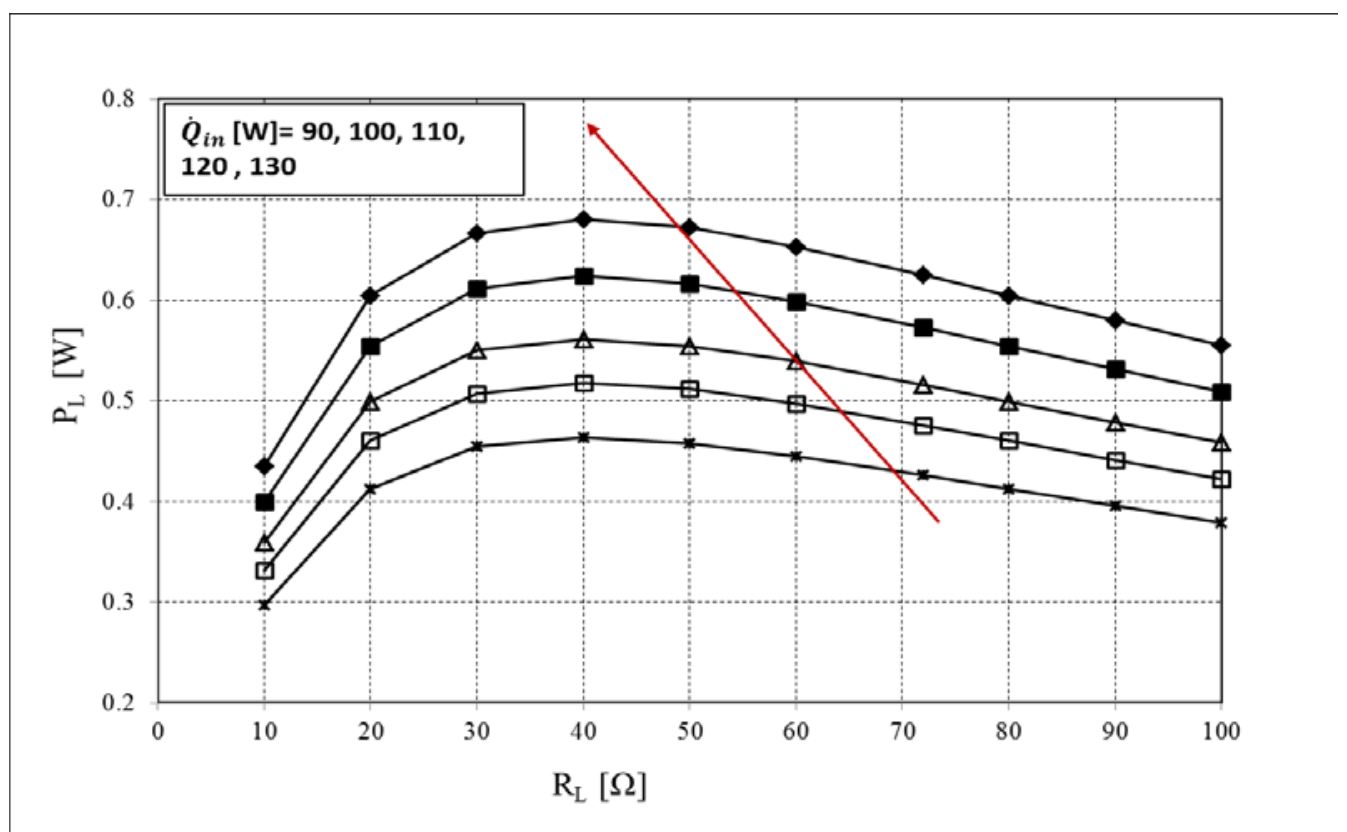

b)

Figure 4-15 Effect of load electrical resistance $($ Rel,L) a) case A b) case B 


\subsubsection{Effect of fin height $\left(H_{\text {fin }}\right)$}

Figure 4-16 illustrates the numerical study of the variations of power supplied to the fan per area of TEG array ( $\left.P_{\text {fan }} / A_{T E G, a}\right)$, net power generated by TEG array $\left(P_{\text {net }} / A_{T E G, a}\right)$ and overall device to environment thermal resistance $\left(\Theta_{\mathrm{th}, \mathrm{T}}\right)$ as a function of height of the fin $\left(\mathrm{H}_{\text {fin }}\right)$ in the heat sink. As the fin height is increased from 20 to $60 \mathrm{~mm}$, the total area of the heat sinks is enlarged by about $167 \%$. Thus, the thermal resistance is decreased by almost $50 \%$ due to the availability of more area for heat transfer.

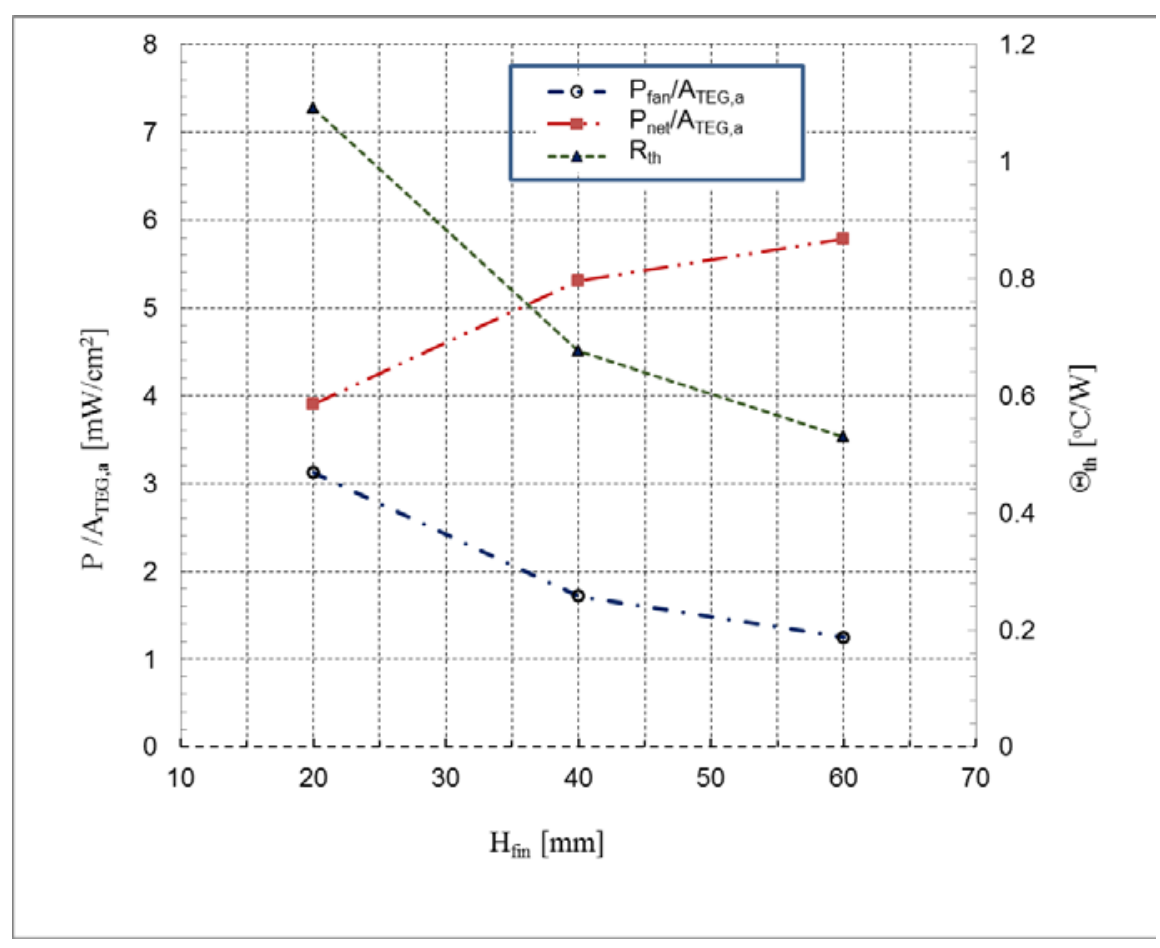

Figure 4-16 Variation of $\mathrm{P}_{T E G} / \mathrm{A}_{\mathrm{TEG}, \mathrm{a}}$ and total thermal resistance $\left(\Theta_{\text {th }}\right)$ as a function of fin height $\left(\mathrm{H}_{\text {fin }}\right)$ 
In addition the pumping power decreases slightly due to decreased friction losses. The velocity of the air in the channel is decreased resulting in lower losses at higher fin heights. Thus, the net power generated by TEG module has also slightly risen.

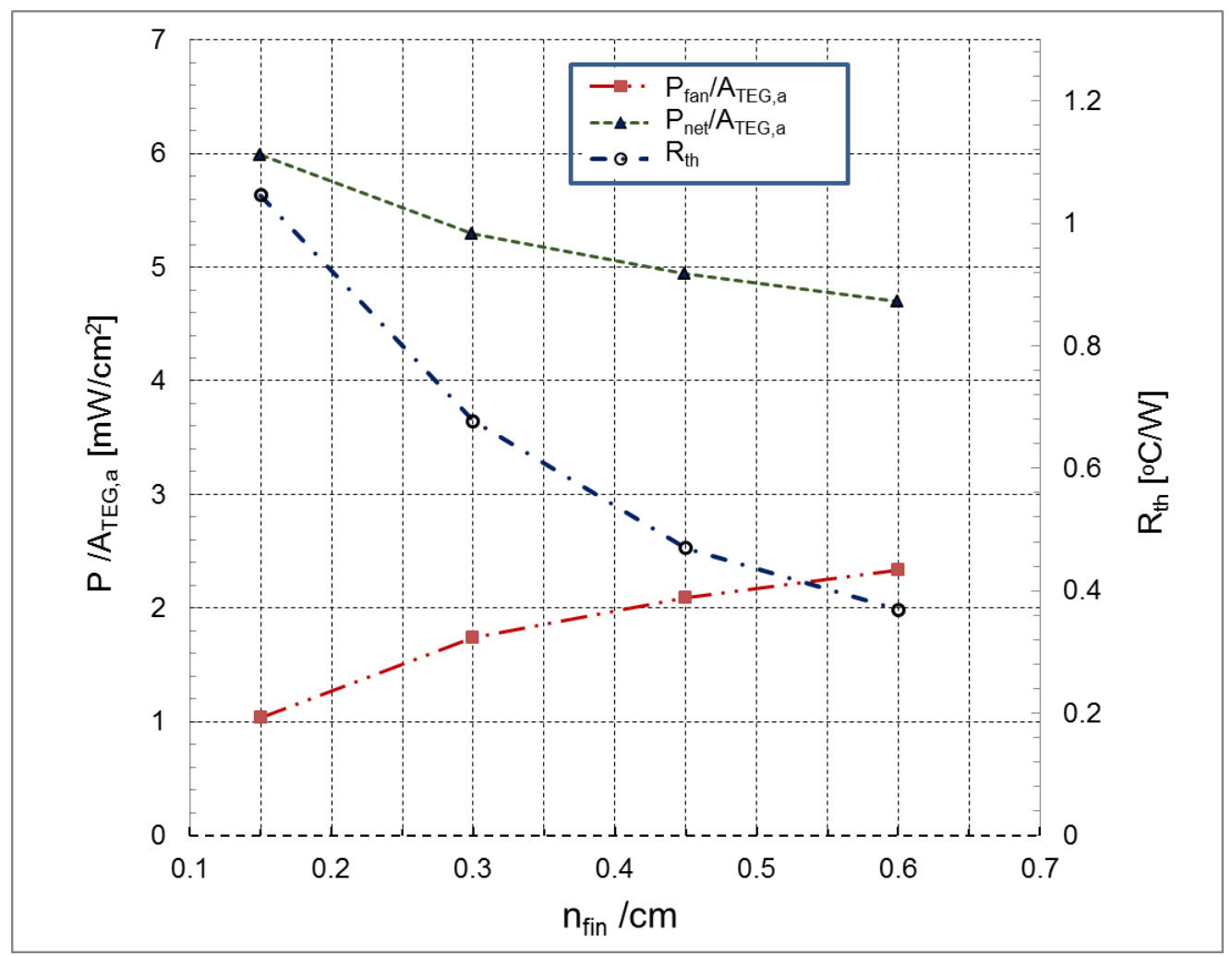

Figure 4-17 Variation of PtEG/Ateg,a and total thermal resistance $\left(\Theta_{\text {th }}\right)$ as a function of fin density $\left(\mathrm{H}_{\text {fin }}\right)$ 


\subsubsection{Effect of fin density $\left(N_{\text {fin }}\right)$}

In Figure 4-17 shows the numerical study of the effect of fin density ( $\left.\mathrm{n}_{\text {fin }}\right)$ on values of pumping power, net power and thermal resistance. As the fin density is

increased from 0.15 to 0.6 , the total area of dissipaters' increases by around $240 \%$ thereby decreasing the total thermal resistance by $70 \%$. However, as the fin density increases, the pressure drops in the dissipater rises requiring more power to be supplied to the fan. As a result, the net power produced by TEG array also declines as shown in Figure 4-17.

However, the system would still be able to provide self-cooling as long as a positive value of $P_{n e t}$ is achieved. Thus, the numerical simulation indicates that a more effective dissipater design in terms of increased surface area would make self-cooling more applicable for range of heat inputs. 


\section{NUMERICAL SIMULATION OF LIQUID COOLING SYSTEM}

\subsection{Introduction}

Previous researches on self-cooling systems mainly concentrated on fan based self-cooling systems. However, for cooling devices where there is a constraint in space or need for strict control of junction temperature, microfluidic system could be a better choice than fan based air cooled systems[83]. Micro heat sink based on microchannel heat sinks could remove high heat fluxes as a result of both expanded area and higher convection heat transfer coefficient (HTC) [84][85] . Nevertheless, fluid flow inside microchannel heat sink systems is associated with larger pressure losses and pumping power as compared to macro fluid systems[86][87]. Thus, they should be designed to operate at an optimum flow rate possible to minimize pumping power while reducing heat transfer resistance [88]. Wojitas et al [89] and Rezania et al [19] have demonstrated the design of compact and effective microchannel heat sink for TEG applications. The aim of their research was to enhance power production from TEG system by using microfluidic cooling with an emphasis on power scavenging from micro-devices.

The integrated microchannel-TEG system could also be applied for self-cooling of the device from which the energy is being extracted. There are, however, two major factors to consider when TEG based self-cooling application system is extended from the concept of TEG power enhancement using microfluidic 
cooling. Firstly, in the experiment [90], the pump power referred to the power needed to move the coolant fluid inside the microchannel. Although, this is the major power loss in the microchannel heat sink based systems, there are also pressure losses in the fluid conduits and secondary heat sink. Therefore, the pump power should consider this effects if the system is to be self- sustaining.

Secondly, for self-cooling application, the junction temperature at the heat source power device is critical For instance, the power consumption for intel based microprocessors follow Moors law [91] and with increasing heat flux the control of operating temperature is of paramount importance and usually has to be kept at the certain threshold level as specified by industrial standards. However, the need for heat management system could be extended to other parts of electronic devices such as memory chips, Integrated circuits (ICs), power converters and switches. Thus, the requirement for allowable maximum junction temperature $\left(T_{j}\right)$ is one of the factors that determine the appropriate ranges of flow rates and heat flux at a power device. For this study, the heat management strategy target is set at maintaining the maximum junction temperature at or below $85^{\circ} \mathrm{C}$.

In this section, the use liquid microchannel for self-cooling of electronic devices is proposed. A numerical model consisting of an integrated microchannel heat sink and TEG module (Figure 5-1) has been made to study the power generated by the TEG module and the power requirement for moving coolant fluid for range of heat flux input. The model has been validated against existing literature on power 
generation and coolant pumping power. The feasibility of self-cooling in terms of net power and control of the junction temperature of heat source device is investigated.

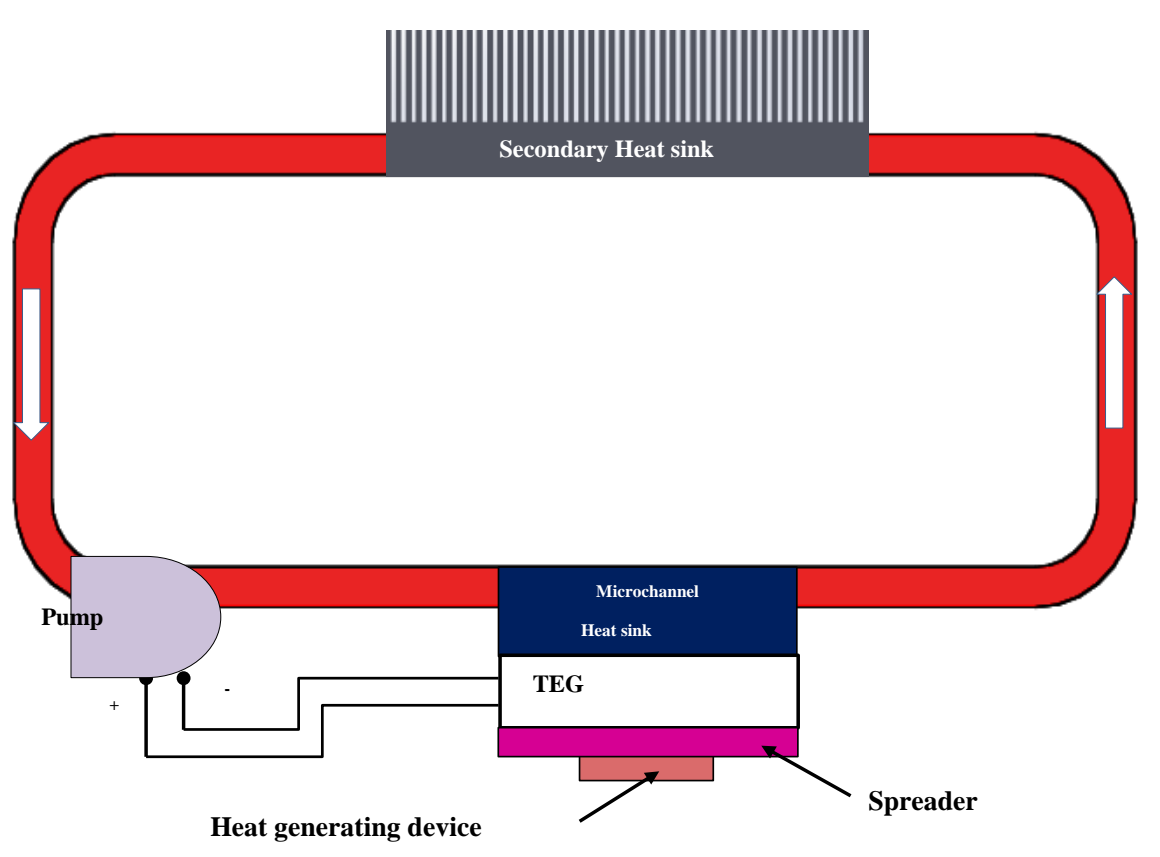

Figure 5-1. A schematic diagram of a microchannel heat sink based self-cooling system

\subsection{Governing equations}

A TEG module generates electric potential $\left(V_{s b}\right)$ when a temperature difference is maintained between the hot and cold sides of the TEG and its magnitude is proportional to the difference in Seebeck coefficient $(\alpha)$ of the two pairs of TEG elements. The pairs of the TEG elements are designated as $p$ and $n$ elements. The power generated from TEG module can be expressed as Eq.5.2.1: 


$$
P_{g e n}=V_{s b} R_{e q}=\left[\frac{2 n_{p n}\left(\alpha_{p}-\alpha_{n}\right)\left(T_{h s}-T_{c s}\right)}{R_{e, p}+R_{e, m}}\right]^{2} R_{e, p}
$$

where $T_{h s}$ and $T_{c s}$ are the cold and hot side temperature of the TEG module, $\alpha_{P}$ and $\alpha_{N}$ are the Seebeck coefficients of the $p$ and $n$ elements respectively and $n_{p n}$ is the number of $p$ and $n$ elements in a TEG module and $R_{e q}, R_{e p}$, and $R_{e, m}$ are the equivalent total electrical resistance, external electric resistance (pump) and the internal TEG module electric resistance respectively. The electrical resistance model consisting of electrical resistances inside TEG module and the load is depicted in Figure 5-2a.

The pumping power required to circulate the cooling medium is given as:

$$
P_{\text {pump }}=\dot{V} \Delta P_{T}
$$

where $\dot{V}$ the volumetric flow rate and $\Delta P_{T}$ is the total pressure loss in the system.

The flow inside microchannel heat sink with an inlet and outlet plenum has sudden expansion from inlet port to the inlet plenum and sudden contraction from outlet plenum to the outlet port. There is pressure loss associated with the flow inside the microchannels. The change of cross-section from inlet plenum into the microchannels and from the microchannels to the outlet plenum also results in pressure losses. In addition, there are pressure losses in fluid conduits, losses 
associated with internal hydraulic resistance in the pump and air-side secondary heat sink.

A single phase flow could be divided into two regions of fluid flow regime. At the inlet condition, the fluid has a developing velocity boundary layer up to the region where the wall effects are dominating resulting in fully developed velocity profile. For laminar flow, the hydrodynamic entrance length, $L_{e n t}$, can be formulated as:

$$
L_{\text {ent }}=0.05 \operatorname{Re}_{D} D_{h}
$$

where $D_{h}$ is the hydraulic diameter of channel and $R e_{D}$ is Reynolds number based on hydraulic diameter. The pressure drop at the developing region $\Delta P_{\text {ent }}$ is given by the Blasius solution:

$$
\Delta P_{e n t}=\frac{2.66\left(\rho_{f} U_{a v}\right)^{1.5}}{\rho_{f} D}\left(\mu_{f}\left(L_{\min }\right)^{0.5}\right)
$$

where $\rho_{f}$ and $\mu_{f}$ are the density and dynamic viscosity of the fluid and $L_{\min }$ is the minimum of $L_{\text {ent }}$ or $L$ (full length of the channel) and $U_{a v}$ is the average velocity. For fully developed flow, pressure drop for a flow inside a channel is given by the equation:

$$
\Delta P_{l}=4 f\left(\frac{L-L_{e n t}}{D_{h}}\right)\left(\frac{\rho_{f} U_{a v}{ }^{2}}{2}\right)
$$


where $f$ is the fanning friction factor. The equivalent hydraulic resistances are given in Figure 5-2b and described in Eq.5.2.6.

$$
R_{h y, T}=R_{h y, p}+R_{h y, c}+R_{h y, m f}+R_{h y, x}
$$

where $\Delta P_{T}$ is the total pressure head. The equivalent total hydraulic resistance $R_{h y, T}$ in Eq.5.2.6) is the sum of the hydraulic resistances inside the pump ( $\left.R_{h y, p}\right)$, the fluid conduits $\left(R_{h y, c}\right)$, inside the microchannel fins $\left(R_{h y, m f}\right)$ and the air-side hydraulic resistance $\left(R_{h y, x}\right)$. The net power in the system, $P_{\text {net }}$ is the difference between the power generated by TEG module $\left(P_{\text {gen }}\right)$ and the pumping power $\left(P_{\text {pump }}\right)$ as expressed in Eq.5.2.7:

$$
P_{\text {net }}=P_{\text {gen }}-P_{\text {pump }}
$$

For a viable self-cooling application, $P_{\text {net }}$ must be positive so that no external power is necessary to run the cooling system. The performance of self-cooling system could also be described in a thermal model using the total junction to ambient thermal resistance between the heat source and the ambient, $R_{\Theta-a:}$

$$
\Theta_{\Theta, a}=\frac{T_{j}-T_{a}}{Q}
$$


where $T_{j}$ and $T_{a}$ are the junction and ambient temperatures. The junction to ambient thermal resistance, $\Theta_{-j a}$ is the sum of all thermal resistances between the junction temperature of the heat source electronic device and the ambient air as described in Figure 5-2c and Eq.5.2.9:

$$
\Theta_{j a}=\Theta_{j c}+\Theta_{s}+\Theta_{M}+\Theta_{x}+\Theta_{f}+\Theta_{a}
$$

where $\Theta_{j c}, \Theta_{s}, \Theta_{M}, \Theta_{X}, \Theta_{f}, \Theta_{a}$ are the thermal resistance in the electronic device, thermal spreader, TEG module, heat sink, fluid and ambient air respectively.

\subsection{Numerical parameters and modeling}

Figure 5-3 shows the main modeling configurations used in the current numerical study. The first configuration in Figure 5-3b which is based on the experiment by [90] is used to validate the numerical model and model Figure $5-3 \mathrm{c}$ is for later analysis of application of self-cooling.

As shown in the schematics of the self-cooling system, Figure 5-1 and numerical model (Figure 5-3c and d), a microchannel heat sink is used to cool an electronic device. TEG module is assembled between the microchannel heat sink and the device and provides the electrical energy to run a pump. A spreader is utilized to conduct heat from a heat source to the hot side of the TEG module. 
a)

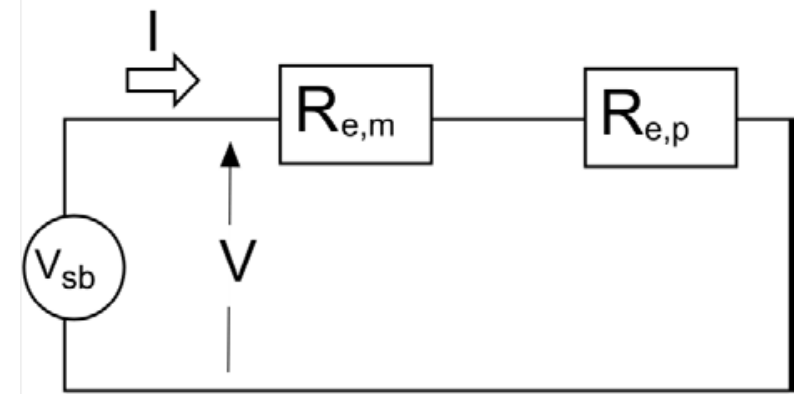

b)
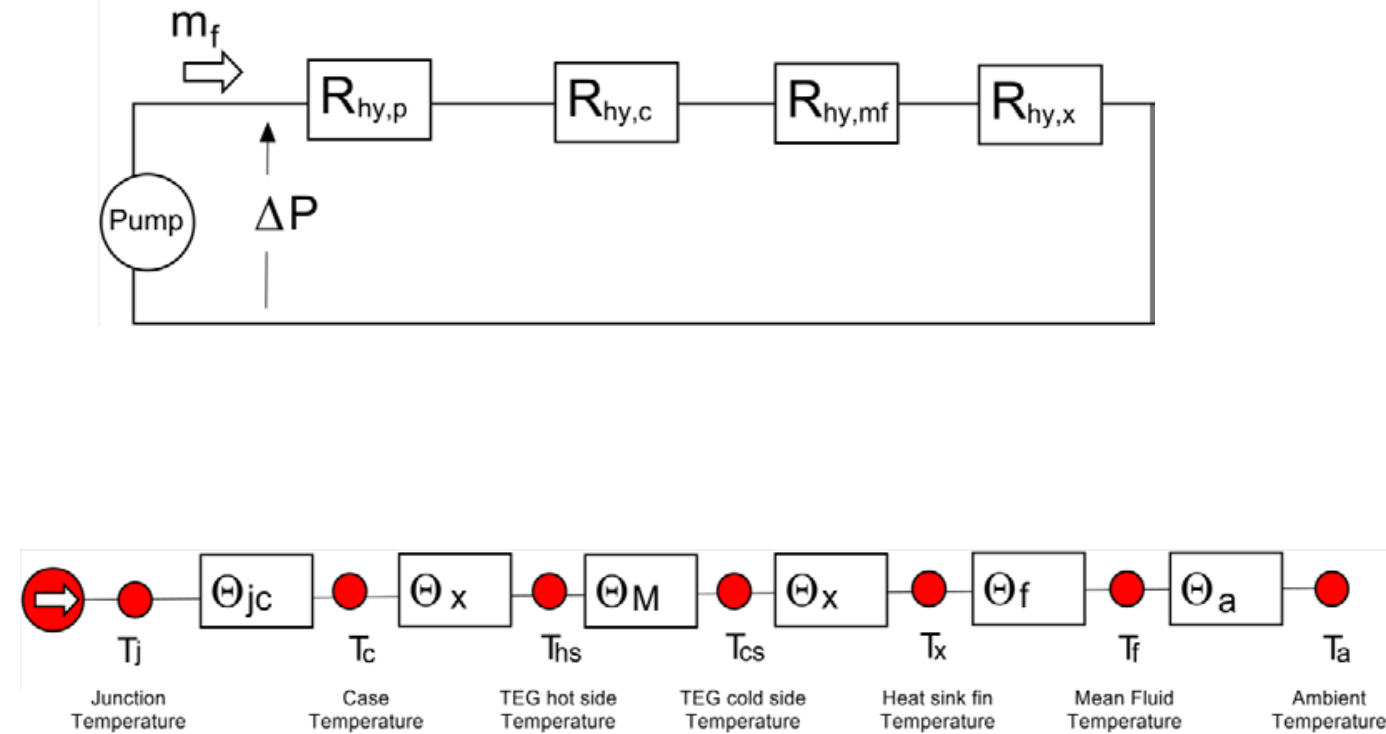

c)

Figure 5-2 Equivalent a) Electrical resistance model of TEG and Pump b) hydraulic resistance model c) thermal resistance model

The following assumptions were made to simplify the modeling of the system and focus on the important system parameters:

(1) Fluid flow is laminar and the fluid is single phase liquid water

(2) Three dimensional and steady state heat transfer and fluid flow 
(3) Temperature independent properties in heat generating device, TEG, microchannel heat sink and fluid

(4) Uniform heat generation in the heat generating device

(5) All surfaces exposed to the surroundings are insulated

For numerical modeling, the governing equations for three dimensional, forced steady state incompressible fluid flow and heat transfer in electronic device, thermal spreader, microchannel fins and water subdomain are solved. The equations for continuity, momentum and energy equations for the fluid (water) can be written as:

Continuity equation:

$$
\frac{\partial u}{\partial x}+\frac{\partial v}{\partial y}+\frac{\partial w}{\partial z}=0
$$

where $u, v$ and $w$ are the fluid velocity components in $x, y$ and $z$ directions respectively.

Momentum equations in $x, y$ and $z$ coordinate:

$$
\begin{aligned}
& \rho_{f}\left(u \frac{\partial u}{\partial x}+v \frac{\partial u}{\partial y}+w \frac{\partial u}{\partial z}\right)=-\frac{\partial P}{\partial x}+\mu_{f} \nabla^{2} u \\
& \rho_{f}\left(u \frac{\partial v}{\partial x}+v \frac{\partial v}{\partial y}+w \frac{\partial v}{\partial z}\right)=-\frac{\partial P}{\partial y}+\mu_{f} \nabla^{2} v
\end{aligned}
$$




$$
\rho_{f}\left(u \frac{\partial w}{\partial x}+v \frac{\partial w}{\partial y}+w \frac{\partial w}{\partial z}\right)=-\frac{\partial P}{\partial z}+\mu_{f} \nabla^{2} w
$$

where $P$ is the pressure field

Energy equation

$$
u \frac{\partial T}{\partial x}+v \frac{\partial T}{\partial y}+w \frac{\partial T}{\partial z}=\frac{k_{f}}{\rho_{f} C_{p}} \nabla^{2} T
$$

The energy equation for solid subdomains (device, thermal spreader, microchannel fins) can be written as:

$$
\nabla^{2} T=0
$$

In the experimental work by [90], a microchannel with twenty rectangular crosssection was fabricated. In their study, a coolant flow rate with Reynolds number (Re) between 63 and 1473 is circulated inside a microchannel using an external pump. The pressure drop across the microchannel heat sink, and the temperature variation of the heat sink and TEG were also recorded.

For the numerical modeling validation with [90] in model Figure 5-3b, a heat flux boundary condition is applied at the bottom of the heat sink to represent a heat generating device. The heat flux at the base of the microchannel heat sink is varied equivalent to $\Delta T_{\text {teg }}$ (temperature difference between the hot and cold sides 
of the TEG) of $10 \mathrm{~K}, 20 \mathrm{~K}, 40 \mathrm{~K}, 60 \mathrm{~K}$ and $80 \mathrm{~K}$ respectively. The coolant flow rate is specified using a uniform velocity inlet condition at the inlet port of the microchannel and a pressure outlet condition at the outlet port. The inlet velocity at the inlet port is varied from 0.059 to $1.27 \mathrm{~m} / \mathrm{s}$ corresponding to flow rate of 0.07 to $1.5 \mathrm{l} / \mathrm{min}$. The fluid inlet temperature was also specified at the inlet port. Adiabatic boundary conditions were applied to the microchannel outside walls.

To investigate the application of self-cooling to microprocessor and similar electronic devices in model (Figure 5-3c and d), the size of the microchip die is set at a foot print area of $15 \times 15 \mathrm{~mm}^{2}$ and $0.5 \mathrm{~mm}$ thickness. A uniform volumetric heat generation is assumed in a microchip device and the heat flux from the chip to the cold heat sink is varied from $15-35 \mathrm{~W} / \mathrm{cm}^{2}$. The coolant flow rate has bounds from $0.007 \mathrm{l} / \mathrm{min}$ to the maximum of $2 \mathrm{l} / \mathrm{min}$ which corresponds to Reynolds number from 69 to 1988 . Thus, the flow remains in laminar region for the whole range of flow rates modeled. As the size of the microchip is less than the TEG module/heat sink assembly, a copper spreader is used. The interior walls between the heat sink, TEG and the heat generating device are set as coupled walls. 


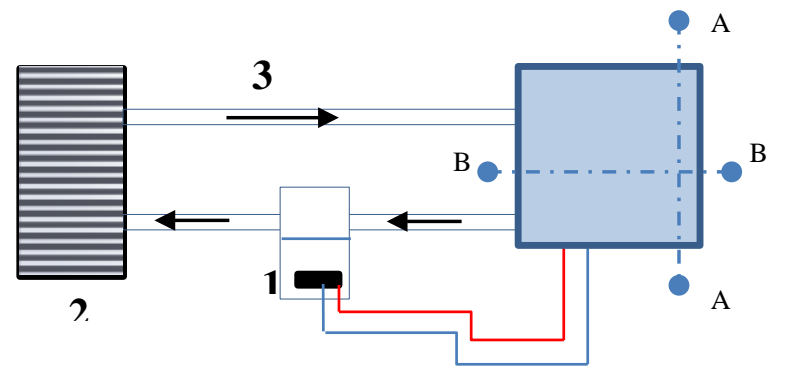

\begin{tabular}{|l|l|}
\hline 1 & Pump \\
\hline 2 & Air-side heat sink \\
\hline 3 & Fluid Conduit \\
\hline 4 & Microchannel Heat sink \\
\hline 5 & TEG \\
\hline 6 & Spreader \\
\hline 7 & Electronic device (Microchip) \\
\hline
\end{tabular}

a) Top view of the system set up

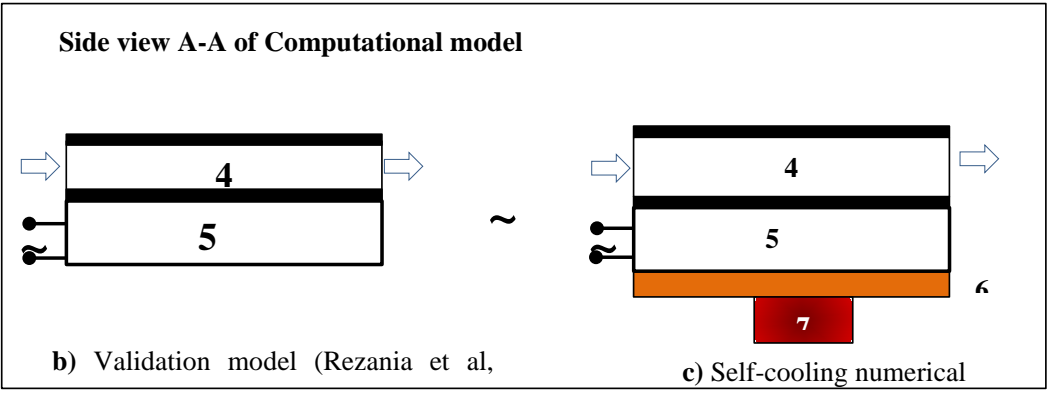

b) Validation model (Rezania et al,

c) Self-cooling numerical

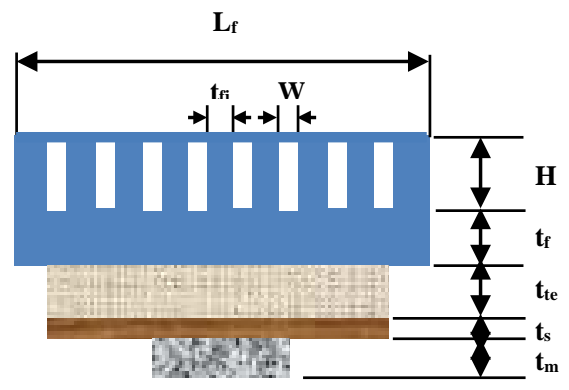

d) Side view B-B of self-cooling

Figure 5-3. Computational model parts and configurations

The major geometrical and simulation parameters are summarized in 


\begin{tabular}{|c|c|}
\hline TEG footprint & $56 \times 56 \mathrm{~mm}^{2}$ \\
\hline TEG thickness, teg & $4.8 \mathrm{~mm}$ \\
\hline Microchannel footprint & $76 \times 56 \mathrm{~mm}^{2}$ \\
\hline Fin height, $\mathrm{H}_{\text {fin }}$ & $1.4 \mathrm{~mm}$ \\
\hline Fin thickness, $t_{\text {fin }}$ & $0.7 \mathrm{~mm}$ \\
\hline Fin base thickness, $\mathrm{t}_{\mathrm{fb}}$ & $1 \mathrm{~mm}$ \\
\hline Channel width, $W_{\text {ch }}$ & $1.4 \mathrm{~mm}$ \\
\hline Fluid density (water) & $998 \mathrm{~kg} / \mathrm{m}^{3}$ \\
\hline Inlet flow rate & $0.059-1.5 \mathrm{l} / \mathrm{min}$ \\
\hline Fluid temperature & $300 \mathrm{~K}$ \\
\hline Numerical model B & \\
\hline Microchip footprint & $15 \times 15 \mathrm{~mm}^{2}$ \\
\hline Microchip thickness, $t_{m}$ & $0.5 \mathrm{~mm}$ \\
\hline Spreader foot print & $56 \times 56 \mathrm{~mm}^{2}$ \\
\hline Spreader thickness, tsp & $1 \mathrm{~mm}$ \\
\hline
\end{tabular}

The Aluminum heat sink and microchip device have $k_{s}=202.4 \mathrm{~W} / \mathrm{m} . \mathrm{K}$ and 137 $\mathrm{W} / \mathrm{m} . \mathrm{K}$ respectively. Numerical simulations were conducted using the commercial Finite volume method (FVM) CFD software ANSYS Fluent ${ }^{\circledR}($ Version 14.5)[80]. A 
second order upwind scheme was used to convert governing equations of momentum and energy to algebraic models by integration of the governing equation and discretizing the applicable formulas based on the conservation of the quantities on the control volume. The Semi Implicit Method for Pressure Linked Equations (SIMPLE) algorithm was used for pressure-velocity coupling. The convergence criteria for scaled residuals were $10^{-4}$ for continuity and velocity balance and $10^{-6}$ for energy balance.

\subsection{Results and discussion}

\subsubsection{Grid dependence study}

Computational model of structured hexahedral grids of 93325, 265776, and 700149 elements designated as grid 1, 2 and 3 respectively were generated for grid dependence study (Figure 5-4a). The variation of temperature along the length of micro channel and the total energy conservation of the system were compared between the different grid sizes.

A dimensionless channel length ratio $(D C L)$ (Eq.5.4.1) is defined to represent temperature distribution along the length of channel \#6. The channels have been designated from number 1 to 10 for half part of the microchannel heat sink starting from left side as shown in Figure 5-4b. 


$$
D C L=\frac{x_{l}}{L_{c h}}
$$

where $x_{l}$ is the length along the channel and $L_{c h}$ is the total length of the channel.
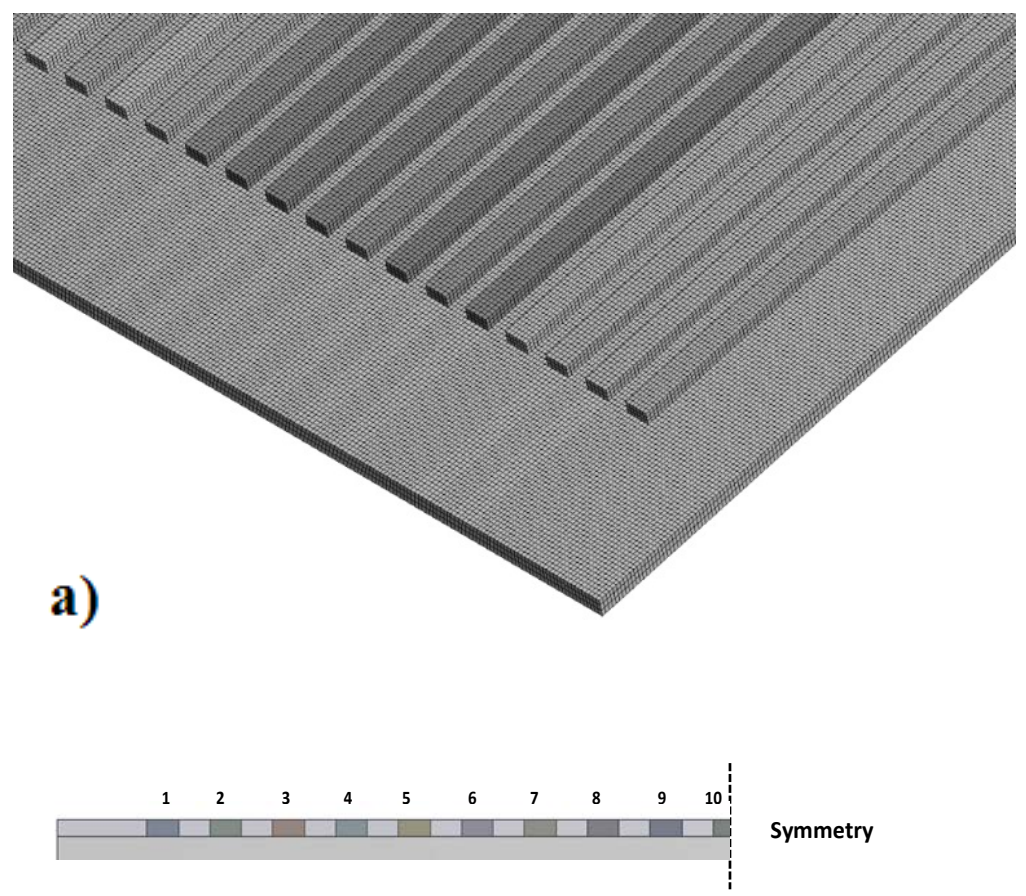

\section{b)}

Figure 5-4. a) Computational grid b) Channel designation for half part of the microchannel heat sink

The simulation result for the variation of temperature along the length of channel for coolant flow rate of $0.076 \mathrm{I} / \mathrm{min}$ and $\Delta T_{\text {teg }}=10 \mathrm{~K}$ is reveled in Figure $5-5$.

It shows that the maximum variation of temperature between the three grid sizes is less than $0.06 \%$ for channel \# 6 indicating that the solution is grid independent for the grid sizes tested. For the total energy balance, the comparison between 
heat applied at the heat sink and the heat absorbed by the coolant fluid was made using Eq.5.4.2. The relative error in energy balance $\Delta Q \%$ is defined as:

$$
\Delta Q \%=\left\|\frac{Q_{\text {flow }}-q_{h s}^{\prime \prime} \times A_{\text {hs }}}{Q_{\text {flow }}} \times 100\right\|
$$

where the heat absorbed by the coolant fluid, $Q_{\text {flow }}$ is given by:

$$
Q_{\text {flow }}=\rho_{f} \dot{V}_{p} \Delta T_{c h, a v}
$$

where $\Delta T_{c h, a v}$ is the average temperature difference between the inlet port and outlet port of the microchannel heat sink. As indicated in Table 5-2, the relative error is not more than $3.79 \%$ for grid 2 and the accuracy changed only by $0.5 \%$ for grid 1 . Thus grid 2 with element size of 265776 is adopted throughout the simulation.

Table 5-2 Comparison between heat applied to the heat sink and heat absorbed by the fluid for different grid sizes for $\Delta$ Tteg $=40^{\circ} \mathrm{C}$

\begin{tabular}{rccc}
\hline \multicolumn{1}{c}{$\dot{V}$} & & $\| \frac{Q_{\text {flow }}-q_{\text {hs }}^{\prime \prime} \times A_{\text {hs }}}{Q_{\text {flow }}} \times 100$ & \\
{$[$ l/min $]$} & Mesh 1 & Mesh 2 & Mesh3 \\
\hline 0.075 & 4.23 & 4.13 & 4.70 \\
0.5 & 5.37 & 5.29 & 9.00 \\
1 & 1.22 & 1.34 & 2.43 \\
1.5 & 4.21 & 4.38 & 10.10 \\
\hline Average & 3.77 & 3.79 & 6.56 \\
\hline
\end{tabular}




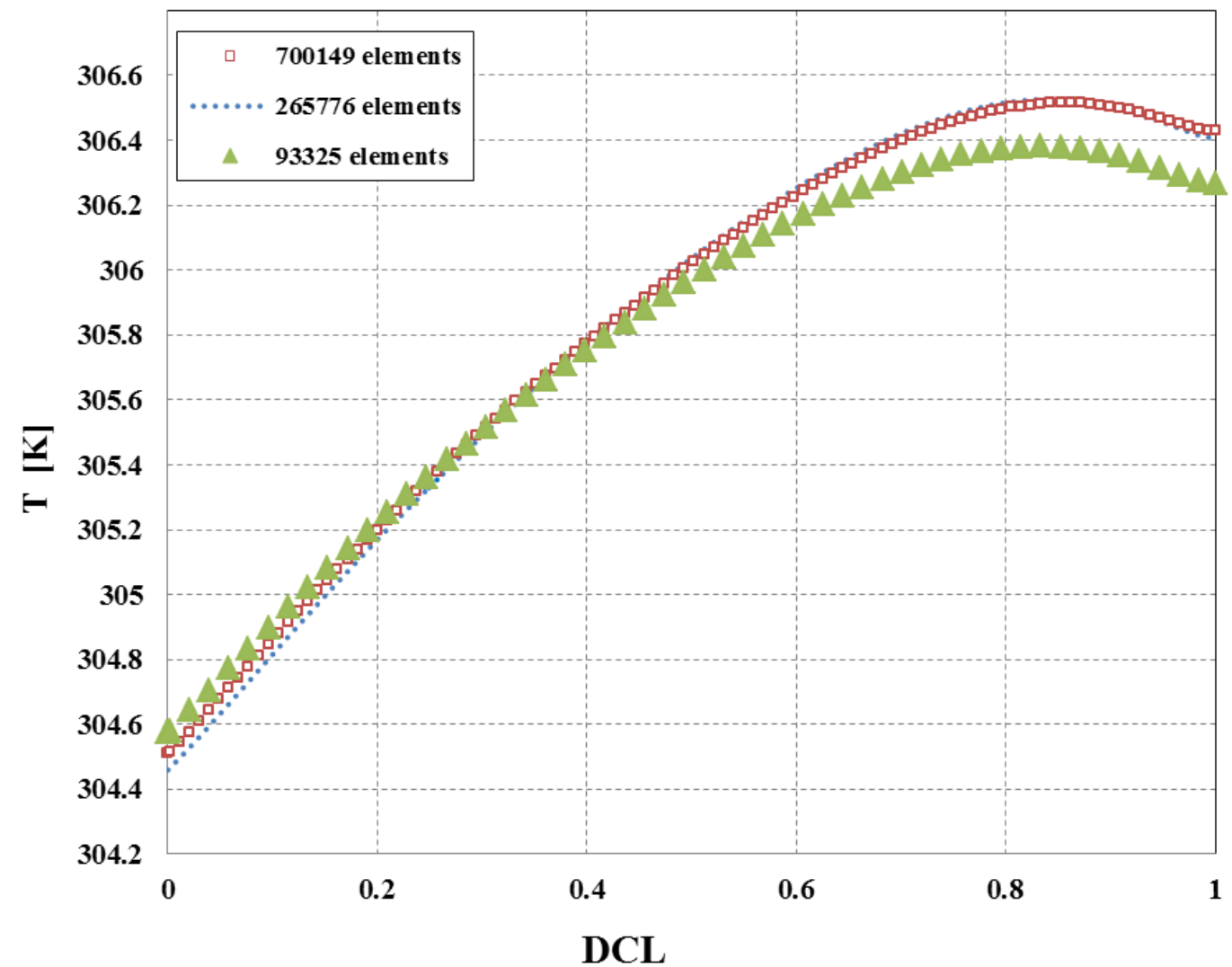

Figure 5-5. The variation of temperature as a function of $D C L$ for channel \#6

\subsubsection{Comparison with experimental data}

The numerical model has been validated against the experimental data (Rezania et al[90]) by comparing pressure and temperature fields. The schematic of the validation model is depicted in Figure 5-3b. The pressure drop across the microchannel was modeled for coolant flow rates in the range of 0.07 and 1.5 I/min. The numerical model revealed a variation of pressure drop with channel location due to the differing flow field in the channels. For the U-type 
configuration of inlet/outlet $(\mathrm{I} / \mathrm{O})$ ports, more pressure drop on the microchannels was also recorded in the experiments in the microchannels close to the heat sink than in the middle of the heat sink. On their numerical study of the effect of $\mathrm{I} / \mathrm{O}$ arrangement on heat sink performance, Chein and Chen [92], have also observed similar effect. Channel \#2 which is close to the heat sink side had the on average more pressure drop (close to $1.55 \mathrm{kPa}$ ) as compared to channel \#10 $(0.77 \mathrm{kPa})$ for the flow rate between 0.07 and $1.5 \mathrm{l} / \mathrm{min}$.

As Figure 5-6 shows, the average pressure loss has been predicted well by the numerical model as compared to the experimental results [90]. At higher volume flow rate, the deviation between numerical results and experimental data was more $(7.6 \%)$ as compared to the lower flow rates. This could be due to an exponential surge in pressure drop reported for the experimental data for channel \#2 at high flow rates.

The change of temperature in the coolant flow for different heat fluxes was also modeled and compared with experimental data as shown in Figure 5-7. There is generally a good correspondence between simulation and experimental results for all the flow rates and temperature differences across TEG $\left(\Delta T_{\text {teg }}\right)$. The coolant fluid temperature demonstrated little variation with flow rate for lower $\Delta T_{\text {teg. }}$ This is due to a higher impact of thermal resistance of the heat sink on coolant temperature at higher $\Delta T_{\text {teg }}$ as has been reported by Wojitas et al [89]and Rezania et al [90]. 


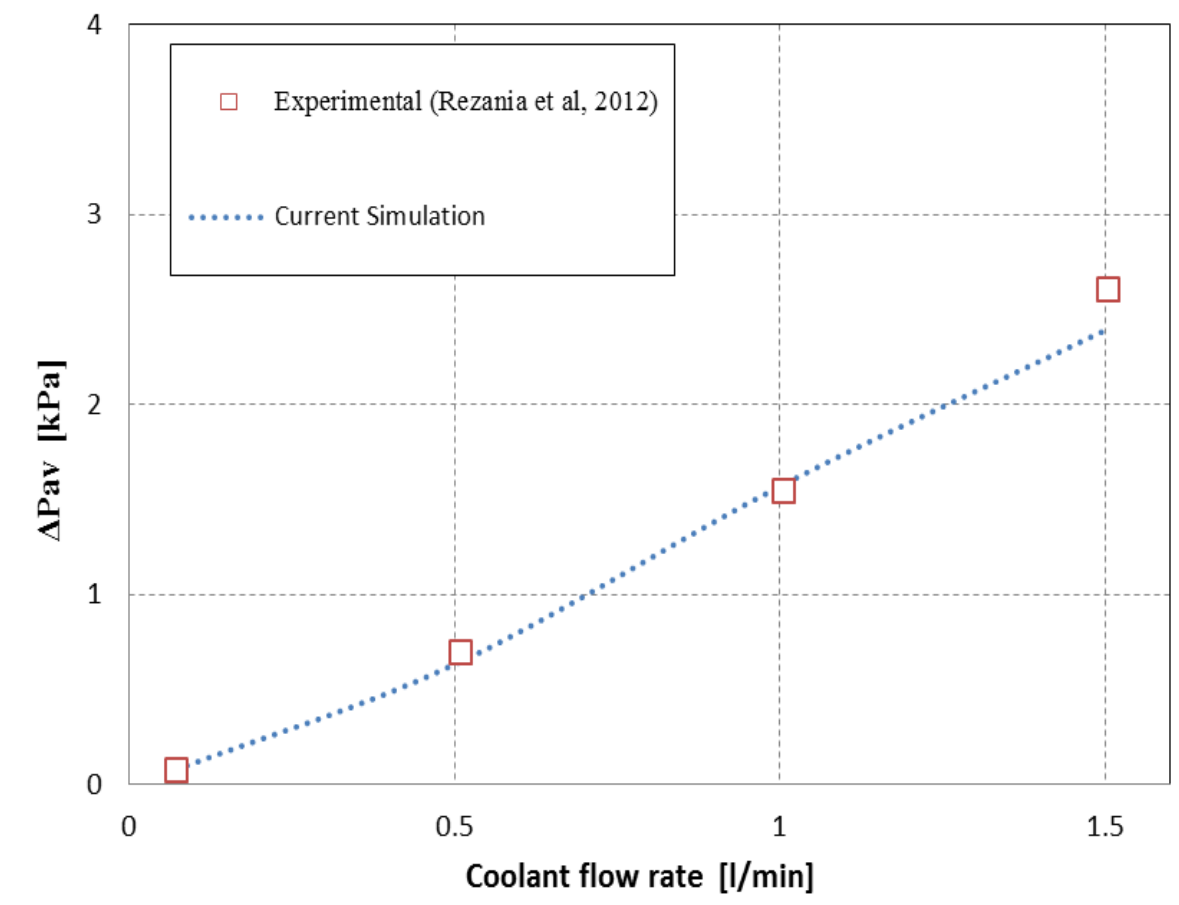

Figure 5-6. Average pressure loss $\left(\Delta P_{a v}\right)$ for the microchannel as a function of coolant flow rate

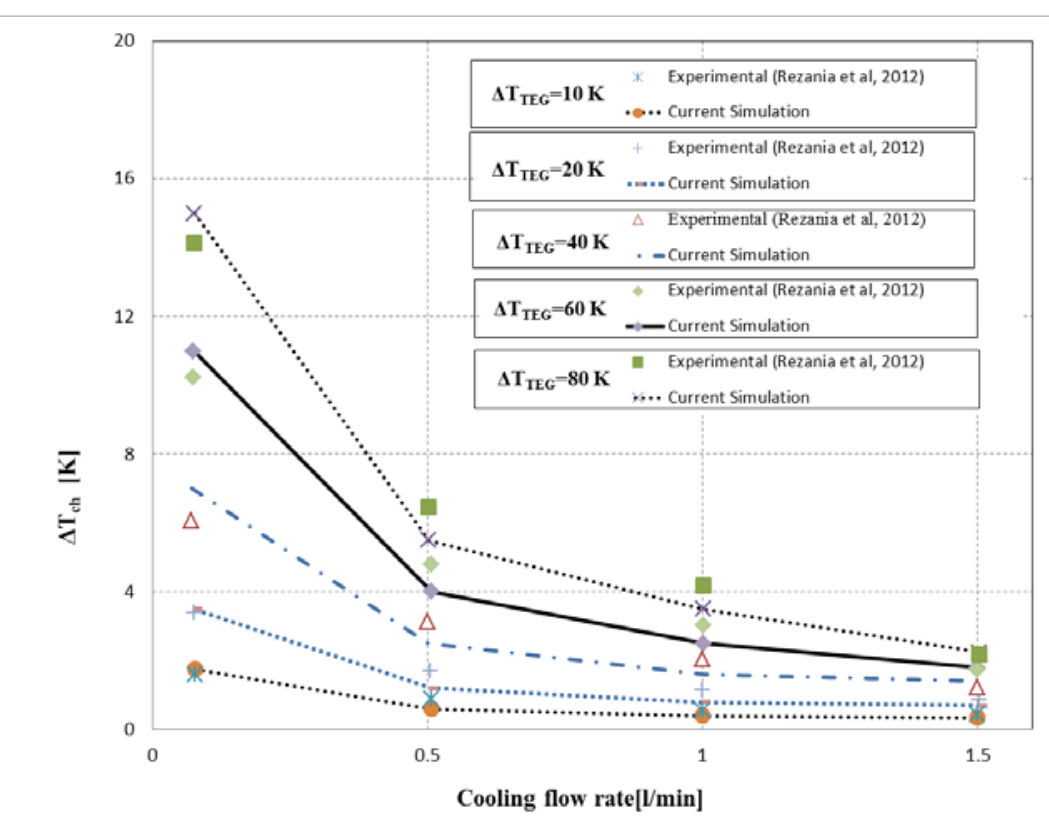

Figure 5-7. Comparison between numerical modelling and experimental data for the coolant temperature difference in channel \#6 between the inlet and outlet port as a function of cooling flow rate 
The numerical model has slightly underestimated the change in coolant flow temperature for lower flow rates and $\Delta T_{\text {teg }}>40 \mathrm{~K}$ as compared to the experimental data. Nevertheless, at lower coolant flow rates and higher heat fluxes, the coolant flow temperature rises at higher rates and the trend has been well simulated by the numerical model.

The net power of the system which is the difference between power generated by the TEG module and the pumping power requirement has been compared between numerical and experimental results. The numerical model has predicted the net power to a very good accuracy as could be observed from Fig 5-6 to 5-8. The raise in coolant flow rate results in lowered temperature at the cold end of the TEG module increasing the power generation rate. However, the pumping power also rises with increased coolant flow rate.

The net power is negative for higher flow rates and lower heat flux (or lower $\left.\Delta T_{\text {teg }}\right)$ which means the system produces less power from the TEG module than it consumes for pumping the coolant fluid. This is due to the combined effect of increased pumping power at higher flow rates and drop in $\Delta T_{\text {teg }}$ as a result of low hot side temperature.

On the other hand, low flow rates at high temperature also tend to decrease the net power owing to higher thermal resistance of the heat sink. Interestingly and as has been pointed out by [90], there is an optimum flow rate which maximizes 
the net power for a certain $\Delta T_{\text {teg. }}$ It can be inferred that a flow rate of $0.5 \mathrm{l} / \mathrm{min}$ maximizes the net power for the range of flow rate and $\Delta T_{\text {teg }}$ simulated and compared with experimental data.

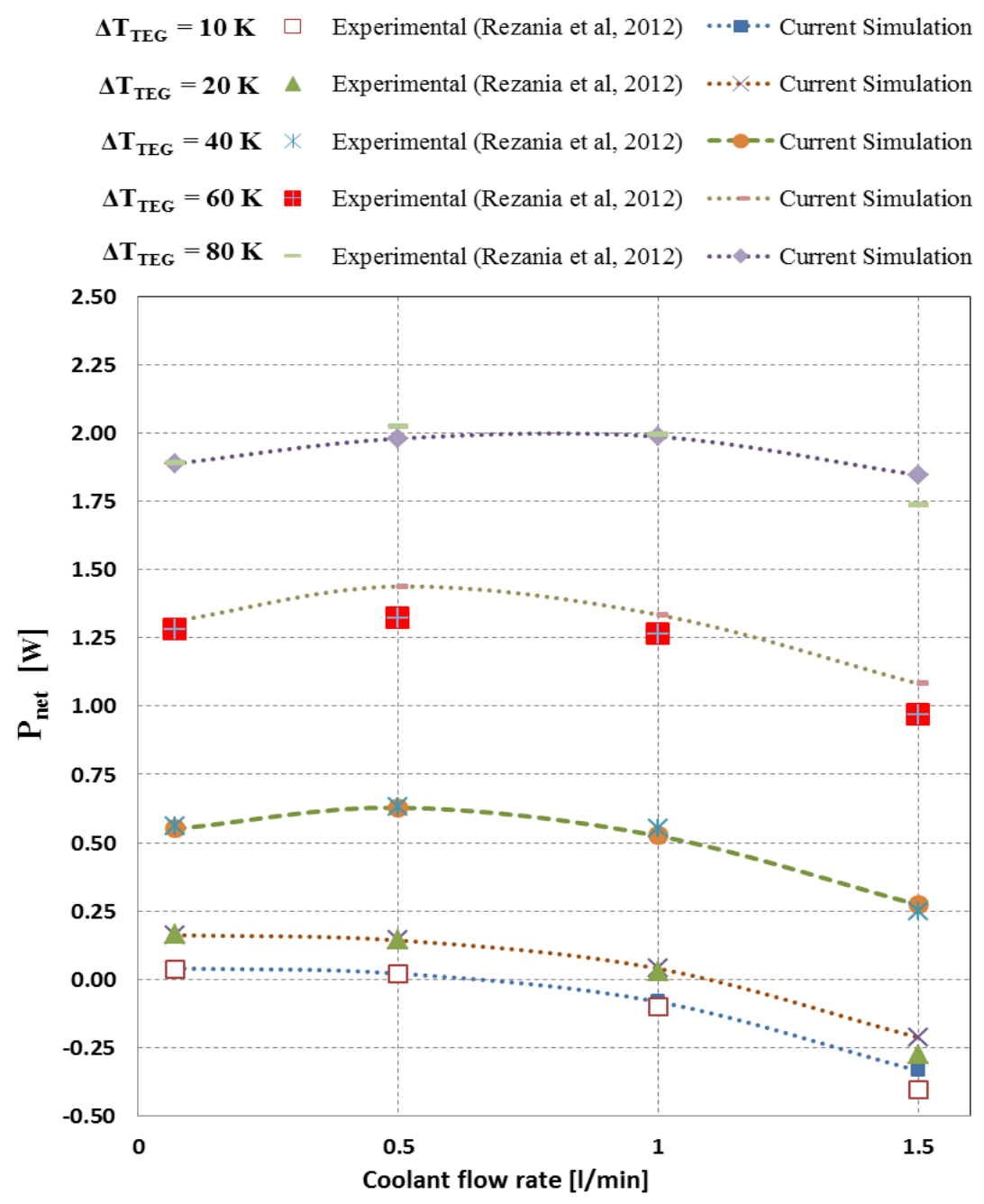

Figure 5-8. The variation of net power with coolant flow rate for different $\Delta T_{\text {teg }}$ 
As expected, a higher temperature difference results in increased net power for a certain coolant flow rate as the TEG generated power is directly proportional to the temperature difference across the TEG,

\subsubsection{Self-cooling application}

The variation of TEG power, pumping power and net power with coolant flow rate has been investigated and the results are shown in Figure. 5-9 for heat flux of 20 $\mathrm{W} / \mathrm{cm}^{2}$. The heat dissipation rate increases with elevated flow rate which could potentially result in lower cold side temperature at the TEG and hence higher $\Delta T_{\text {teg. }}$. For a rise in flow rate from $0.07 \mathrm{l} / \mathrm{min}$ to $0.05 \mathrm{l} / \mathrm{min}$, a $12 \%$ hike in $\Delta T_{\text {teg }}$ is observed. However, from $0.05 \mathrm{l} / \mathrm{min}$ to $1 \mathrm{l} / \mathrm{min}$, the rate of increase of $\Delta T_{\text {teg }}$ was down to only $3 \%$. Similarly, TEG power increased by only $7 \%$ as the flow rate is augmented from $0.5 \mathrm{l} / \mathrm{min}$ to $1 \mathrm{l} / \mathrm{min}$ which is less than (26\% rise) as compared to for a change in flow rate from $0.07 \mathrm{l} / \mathrm{min}$ to $0.5 \mathrm{l} / \mathrm{min}$. On the other hand, fluid pumping power shows an exponential relation with coolant flow rate. The fluid pumping power could go up by as much as $200 \%$ for $0.5 \mathrm{l} / \mathrm{min}$ increase from 1 I/min. Thus, as indicated by Figure. 5-9, the net power from the system grew to the maximum value as the flow rate was raised from $0.07 \mathrm{l} / \mathrm{min}$ to $0.5 \mathrm{l} / \mathrm{min}$ during which the rate of increase of TEG power was more than the pumping power. But after reaching a maximum value at $0.5 \mathrm{l} / \mathrm{min}$, the net power fell exponentially and even achieving negative value at elevated flow rate of $2 \mathrm{l} / \mathrm{min}$. From self-cooling point of view, special consideration must be given to the optimum value of flow 
rate which results in the maximum net positive power. It is also of interest to analyze rate of increase of net power with a rise in heat flux from the heat source device.

As indicated in Figure 5-10a, the net power from the system increases with the heat flux from the device for a given coolant flow rate. With a constant thermal resistance at the heat sink, the hot side temperature of the TEG rises with an increased heat flux resulting in higher power generation.

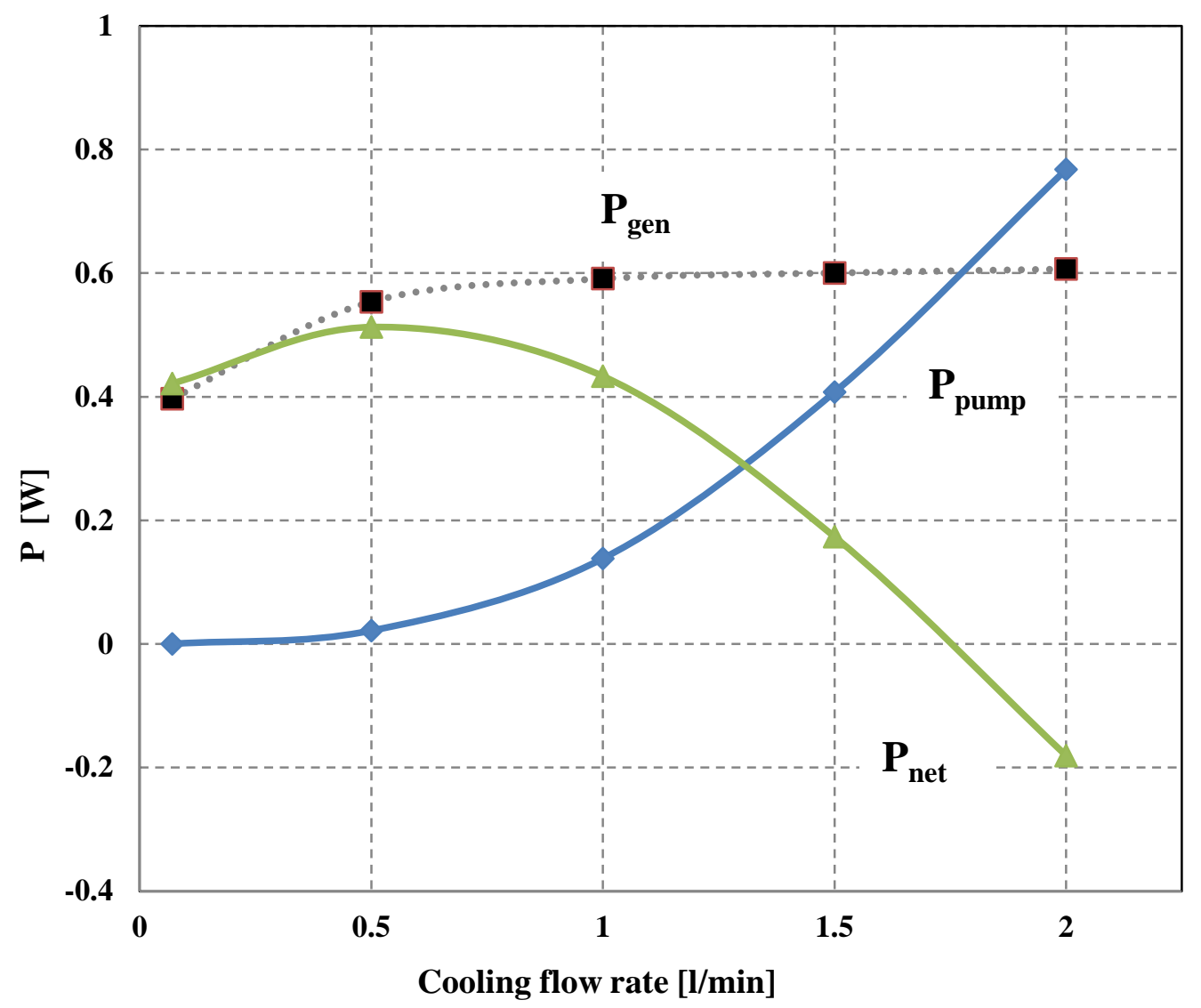

Figure. 5-9. Maximum generated power $\left(P_{\text {gen }}\right)$, pumping power $\left(P_{\text {pump }}\right)$ and net power (Pnet) for $\boldsymbol{q}^{\prime \prime}=20 \mathrm{~W} / \mathrm{cm}^{2}$ 
Figure $5-10 \mathrm{~b}$ shows that the junction temperature has not been greatly affected by the coolant flow rate. In this study, it is calculated that the heat sink thermal resistance is only around $10 \%$ of the total thermal resistance and only changes by around $15 \%$ for an increment of cooling flow rate from $0.5 \mathrm{l} / \mathrm{min}$ to $2 \mathrm{l} / \mathrm{min}$ (Figure. 5-11).

Wojitas et al[89] and Rezania and Rosendhal[93] also noted that the relatively low thermal resistance at the microchannel heat sink compared to the total thermal resistance could be the reason for the minimal effect of the coolant flow rate on the hot side temperature of TEG module.

Nevertheless, an increased flow rate entails a surge in pumping power .It could be inferred from Figure 5-10 $\mathrm{a}$ and $\mathrm{b}$ that for heat flux $15 \mathrm{~W} / \mathrm{cm}^{2}$ and $20 \mathrm{~W} / \mathrm{cm}^{2}$, the junction temperature has been kept below $85{ }^{\circ} \mathrm{C}$ for flow rate between 0.5 $\mathrm{l} / \mathrm{min}$ to $2 \mathrm{l} / \mathrm{min}$. But for the lower heat flux of $15 \mathrm{~W} / \mathrm{cm}^{2}$, the net power is not enough for self-cooling application due to more pumping power requirement than the TEG generated power. 

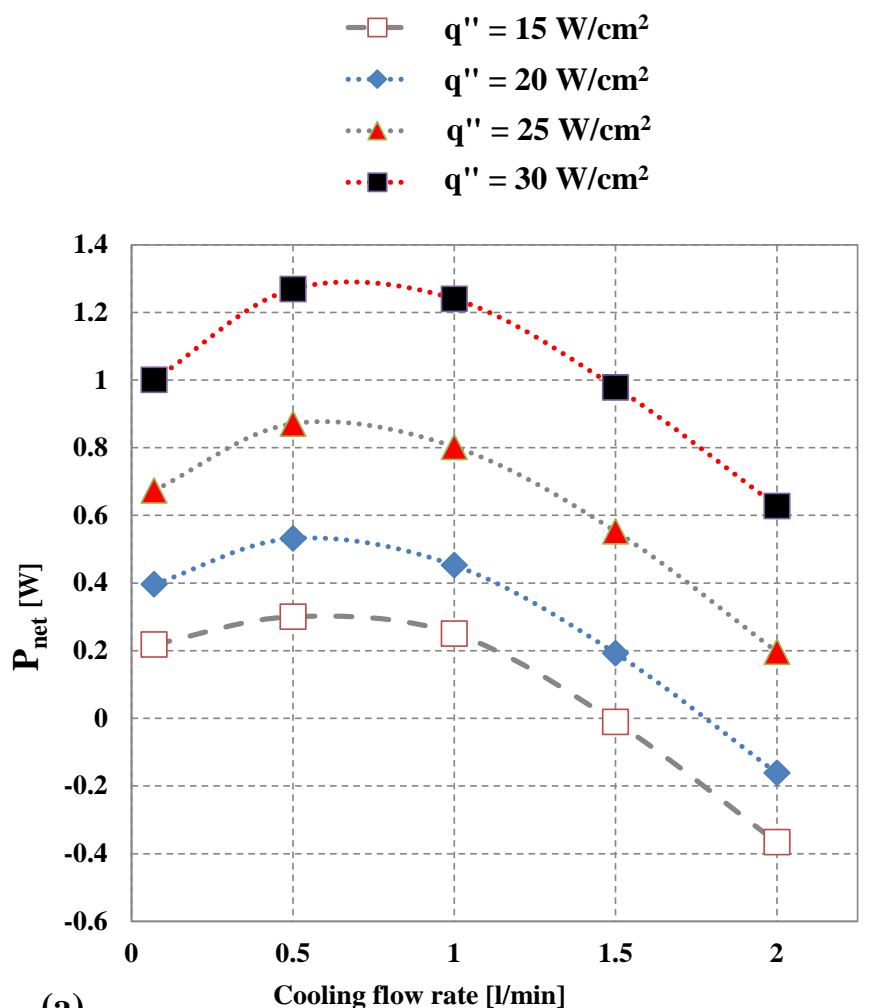

(a)

Cooling flow rate $[1 / \mathrm{min}]$

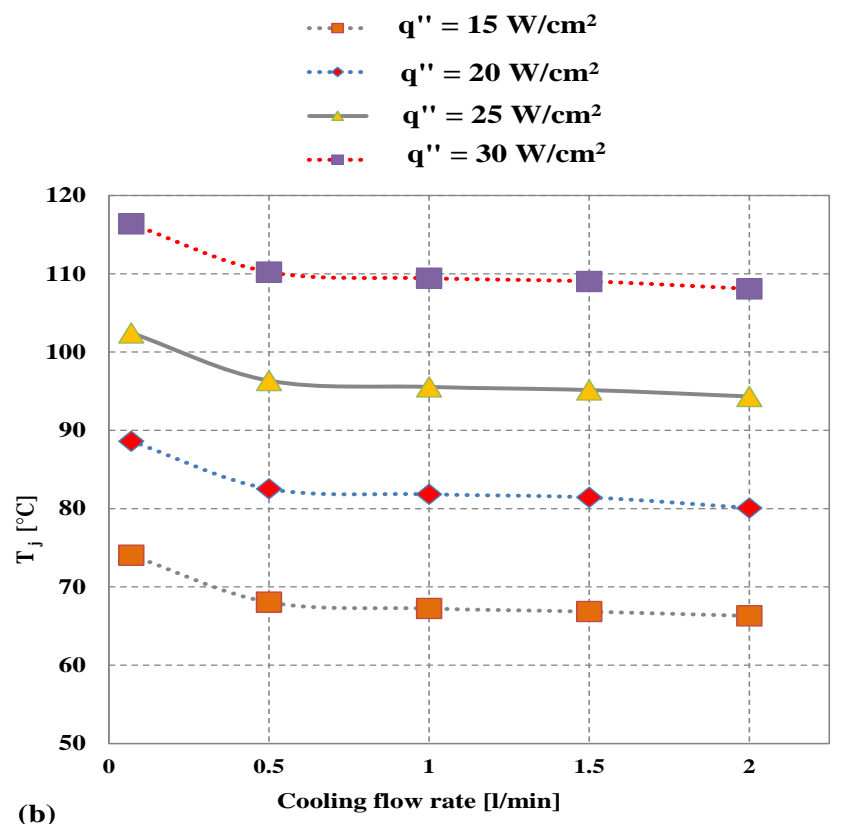

Figure 5-10. Variation of net power and junction temperature with heat flux and cooling flow rates 


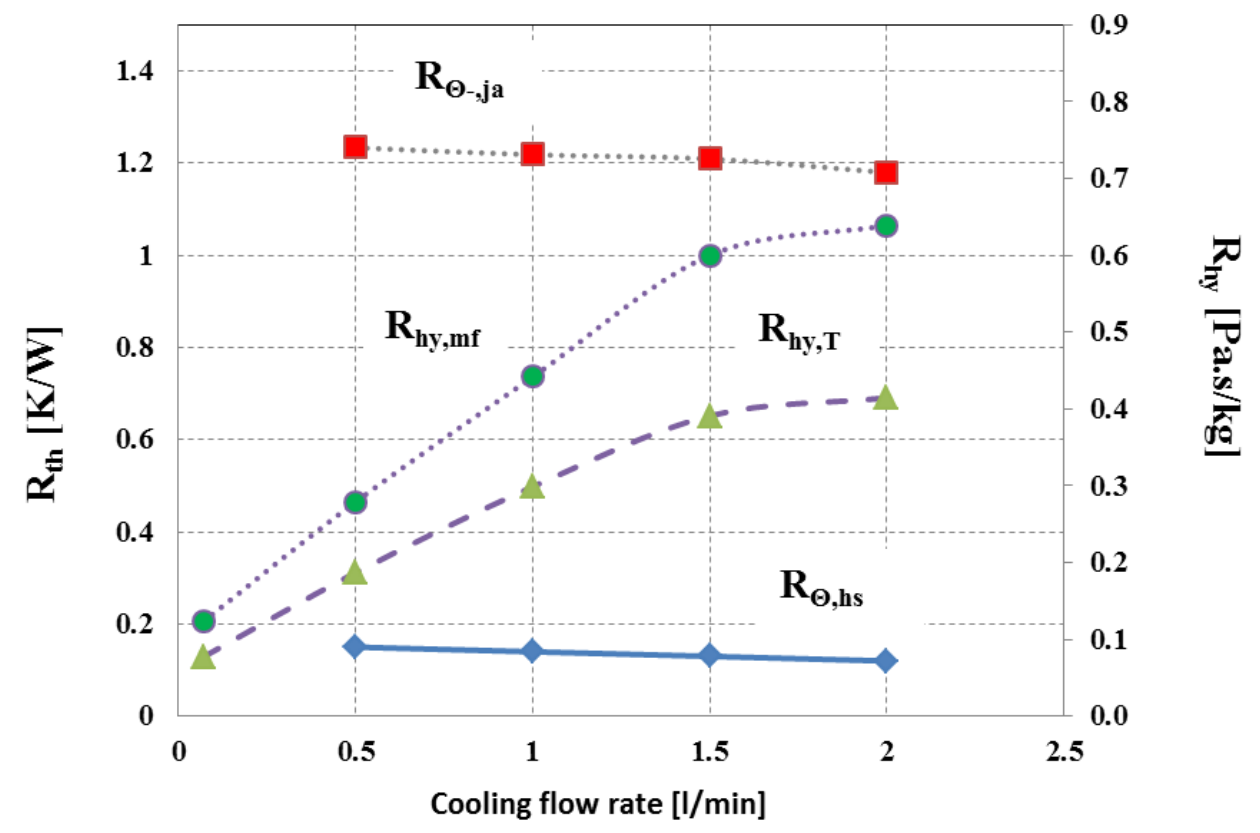

Figure. 5-11. Variation of thermal and hydraulic resistance with cooling flow rate

The system, however, could potentially be self-cooling for heat flux and flow rate range of $15 \mathrm{~W} / \mathrm{cm}^{2}$ to $20 \mathrm{~W} / \mathrm{cm}^{2}$ and 0.075 to $1 \mathrm{l} / \mathrm{min}$ respectively. The maximum net power for junction temperature of less than $85^{\circ} \mathrm{C}$ is achieved at $0.5 \mathrm{l} / \mathrm{min}$. The performance of self-cooling system is also modeled by comparing its performance with commercially available micro pumps. A Micro centrifugal liquid pump ( Model M400S from TCS Micropumps Ltd )was selected for the comparison and the performance data was extracted from the manufacturer provided data sheet[94]. As could be inferred from Figure. 5-12, the pressure loss for the modeled flow rate is significantly less than the pressure difference that could be provided by the pump. 


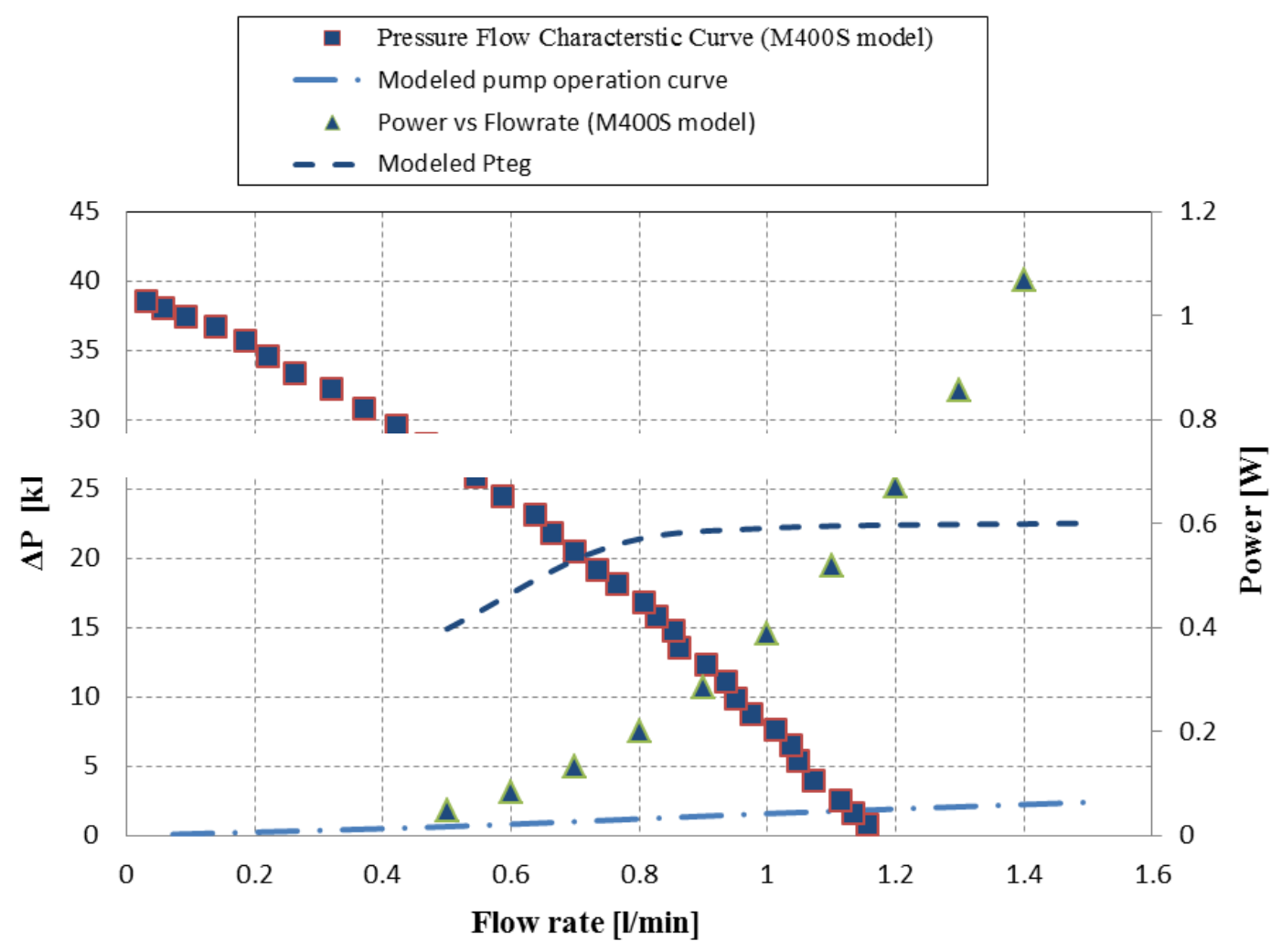

Figure. 5-12. Comparison of modeled Pressure flow characteristic and power requirement with commercial micropump

Thus, the pump could easily furnish the necessary pressure to move the coolant through the cold heat sink. For the power requirement by the pump, the TEG module can provide enough power up to flow rate of $1.2 \mathrm{l} / \mathrm{min}$ for the type of pump modeled. Thus, self-cooling has a potential for providing power to cool devices at an optimum junction temperature provided that a proper matching of cooling flow rate is made to the heat flux from the heat source electronic device. 


\section{DYNAMIC MODELING AND ANALYSIS OF SELF-COOLING SYSTEM}

\subsection{Introduction}

In this section, a compact one-dimensional dynamic model of self-cooling system is developed. The model is constructed by representing the system using thermal elements of thermal resistance and capacitance. In the first stage, each body is depicted using its equivalent thermal capacitance and temperature. The model is then modified by using segmented sub-bodies to represent each body thereby improving the accuracy of the model. The model is then implemented in MATLAB/Simulink software and the results are compared against experimental and three dimensional numerical models.

The main types of modeling thermoelectric generation could be divided into two major types. The first type is design model which constitutes a complete or partial representation of the geometry of the physical model. The second type is a dynamic model which represents the whole system in terms of electrical equivalent model. The model for the first type of modeling strategy has already been shown in previous chapters. In this chapter, a dynamic model for thermoelectric generation based self-cooling system is developed. The model has thermal and electrical subparts. The thermal model is represented using thermal elements. The two most important passive thermal elements which could be used to depict the dynamic behavior of thermal systems are thermal 
capacitance and thermal resistance. Both thermal elements are normally associated with spatially distributed systems but could also be used to describe dynamic behavior in thermal systems using the lumped model approach.

\subsubsection{Thermal capacitance}

For a system which does not exhibit change of phase and within a reasonable range of temperature, the net heat flow into a body at time $t$ is given as:

$$
q_{\text {net }}(t)=q_{\text {in }}(t)-q_{\text {out }}(t)
$$

While the net heat supplied to the body from time to is:

$$
q_{\text {net }}(\lambda)=\int_{t_{0}}^{t_{1}}\left(q_{\text {in }}(\lambda)-q_{\text {out }}(\lambda)\right) d \lambda
$$

Assuming that the temperature change in a body without a phase change is directly proportional to the heat supplied to the body in time range $\lambda$, the proportionally constant is given by thermal capacitance $\mathrm{C}$

$$
C=\frac{T(t)-T\left(t_{0}\right)}{\int_{t_{0}}^{t_{1}}\left(q_{\text {in }}(\lambda)-q_{\text {out }}(\lambda)\right) d \lambda}
$$

where the unit of $C$ is $\mathrm{J} / \mathrm{K}$. 
Rearranging the terms and differentiating Eq. 6.1.3, the rate of temperature change $(\dot{\theta})$ is expressed as function of the instantaneous net heat into the body by:

$$
\dot{\theta}=\frac{1}{C}\left[q_{\text {in }}(t)-q_{\text {out }}(t)\right]
$$

\subsubsection{Thermal resistance}

For a heat flow from one point to another point in a body, the heat flow $q(t)$ could be described by the temperature change between the two point and thermal resistance in the path between the points as:

$$
q(t)=\frac{1}{\Theta}\left[T_{1}(t)-T_{2}(t)\right]
$$

Where $\mathrm{R}$ has a unit of $(\mathrm{K} / \mathrm{W})$

\subsubsection{Thermal sources}

A thermal source could be termed as heat source or sink depending on whether the thermal source adds or removes heat from a body respectively. 


\subsection{Electric circuit analogy for lumped body analysis}

In a lumped body thermal analysis, the thermal properties of body are assumed to be represented by a single value. This type of approximation is more applicable when the conduction of heat inside the body (high thermal conductivity) is of higher magnitude or faster as compared to heat loss from the body. The Biot number (Bi) which is defined as the ratio of conductive heat resistance to the convective heat resistance across the body boundaries is usually utilized to analyze if the lumped body approximation is valid. Usually a body or part of a body with Biot number less than 0.1 can be approximated with lumped body analysis.

\subsubsection{Heat source device}

The three dimensional heat equation inside the heat source device in Cartesian coordinate could be given by:

$$
d m C \frac{d T}{d t}=k\left(d A_{x} \frac{d^{2} T}{d x^{2}}+d A_{y} \frac{d^{2} T}{d y^{2}}+d A_{z} \frac{d^{2} T}{d z^{2}}\right)+\dot{q}_{\text {in }} \quad 6.2 .1
$$

The equation could be simplified by representing the direction which is in contact with the TEG module using conduction resistance and surfaces exposed to the atmosphere by convection resistance. 
For the body represented by Figure. $6-1$, the following assumptions could be made.

a) The temperature of the device is represented by $T_{d}$ which is the average temperature of the device

b) The body is connected to the hot side of TEG one side where conduction heat transfer takes place and the equation for conduction in x-direction is given by:

$$
q_{x}=k d A_{x} \frac{d^{2} T}{d x^{2}}
$$

c) The lateral sides exposed to the ambient air at $T_{\text {env }}$ and heat is lost convectively to the surrounding environment

$$
q_{y}=k d A_{x} \frac{d^{2} T}{d y^{2}}=h A_{y}\left(T-T_{e n v}\right)
$$




$$
q_{z}=k d A_{z} \frac{d^{2} T}{d z^{2}}=h A_{z}\left(T-T_{e n v}\right)
$$

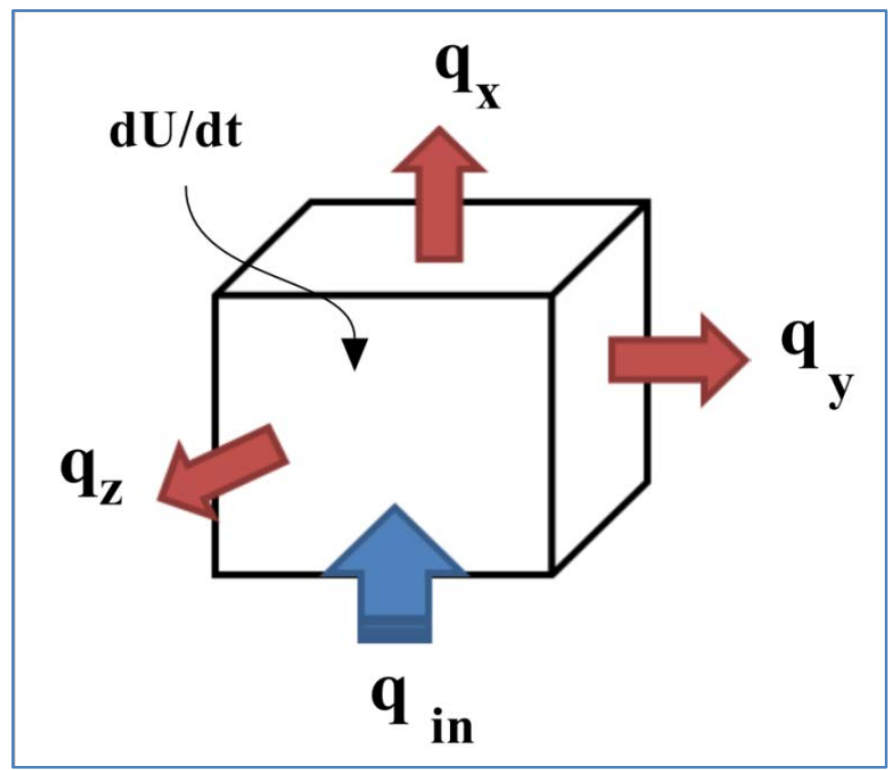

Fiqure. 6-1 heat transfer in heat source

Based on the assumptions made above, an equivalent thermal circuit diagram based on electrical circuit analogy could be made as shown in Figure. 6-2. 


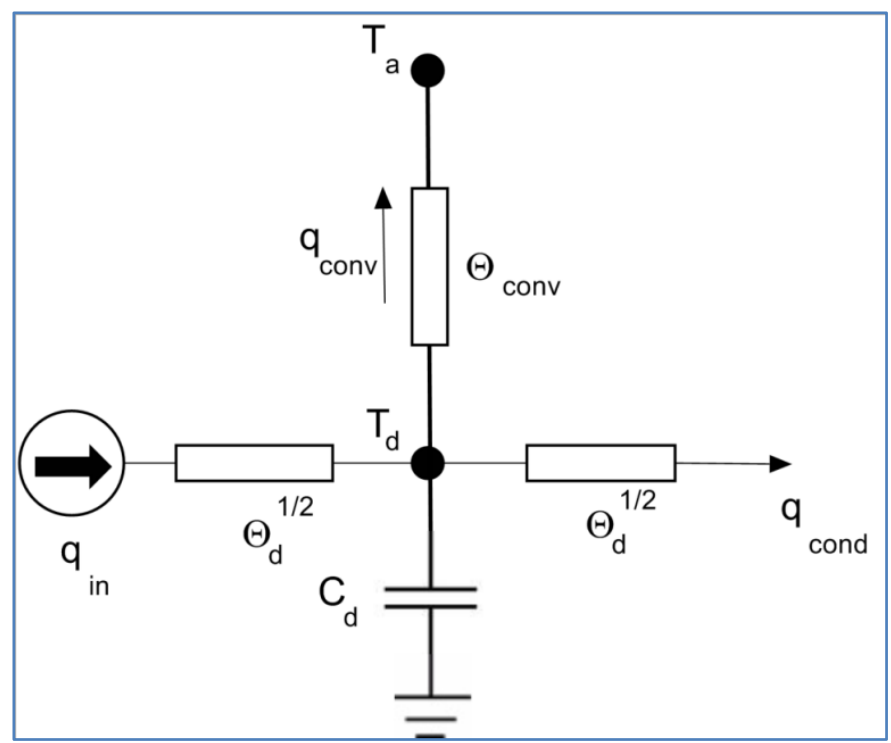

Figure. 6-2 Electrical circuit analogy of heated device

The heat input to the device is given as $\mathbf{q}_{\text {in. }}$ The change of internal energy, $d U / d t$ can be represented by using heat capacity $C_{d}$ which is expressed as

$$
C_{d}=\frac{q_{\text {net }}}{\dot{\theta}_{d}}
$$

where $\dot{\theta}_{d}$ is the rate of temperature change and $\mathrm{q}_{\text {net }}$ the instantaneous net heat inside the device.

The convection thermal resistance on the sides exposed to the ambient air is given by:

$$
\Theta_{\text {conv }}=\frac{1}{h A_{L}}
$$


Where $A_{L}$ is the total area of lateral sides exposed to the atmosphere.

As the average temperature of the device, $T_{d}$ is considered to act the center of the device, the conduction thermal resistance is divided into two equal parts, $\Theta_{d}^{1 / 2}$, each representing half part of the device.

The conduction thermal resistance is given as:

$$
\Theta_{d}^{1 / 2}=\frac{L_{d}^{1 / 2}}{k_{d} A_{d}}
$$

Where $L_{d}^{1 / 2}$ and $A_{d}$ is the length of half part of the device in the direction and area of TEG module respectively and are in the direction where the device conducts heat to the TEG module. The heat conducted to the TEG module is given as qcond.

\subsubsection{Thermoelectric module (lumped model)}

A thermoelectric module consists of number of TEG modules connected in series by copper solder and contained between thermally conductive and electrically insulated ceramic plates. The simulation of numerous P-N pairs inside each module in an array would result in high computational complexity in terms of grid size as well as computational time [81]. Thus, the TEG module is modeled as TEG cuboid consisting of three parts as shown in Figure. 6-3. The first part 
represents $\mathrm{P}$ and $\mathrm{N}$ semiconductor pellets with an air gap and copper conductors. The second and third parts are the top and bottom ceramic plates.

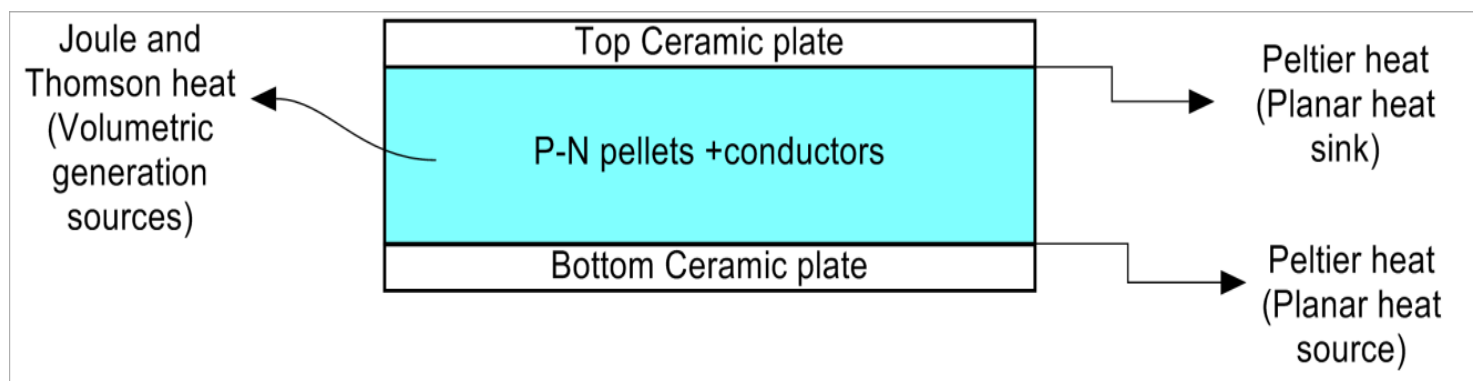

Figure. 6-3 TEG module compact model

The electrical circuit analogy of the TEG module has also three parts. The first part represents the bottom ceramic plate (Figure. 6-4) which is in contact with the heat source device. As the average temperature of the bottom ceramic plate, Ths is considered to act the center of the ceramic. It also represents the hot side temperature of the TEG module. The conduction thermal resistance is divided into two equal parts, $\Theta_{c e r}^{1 / 2}$, each representing half part of the ceramic plate. 


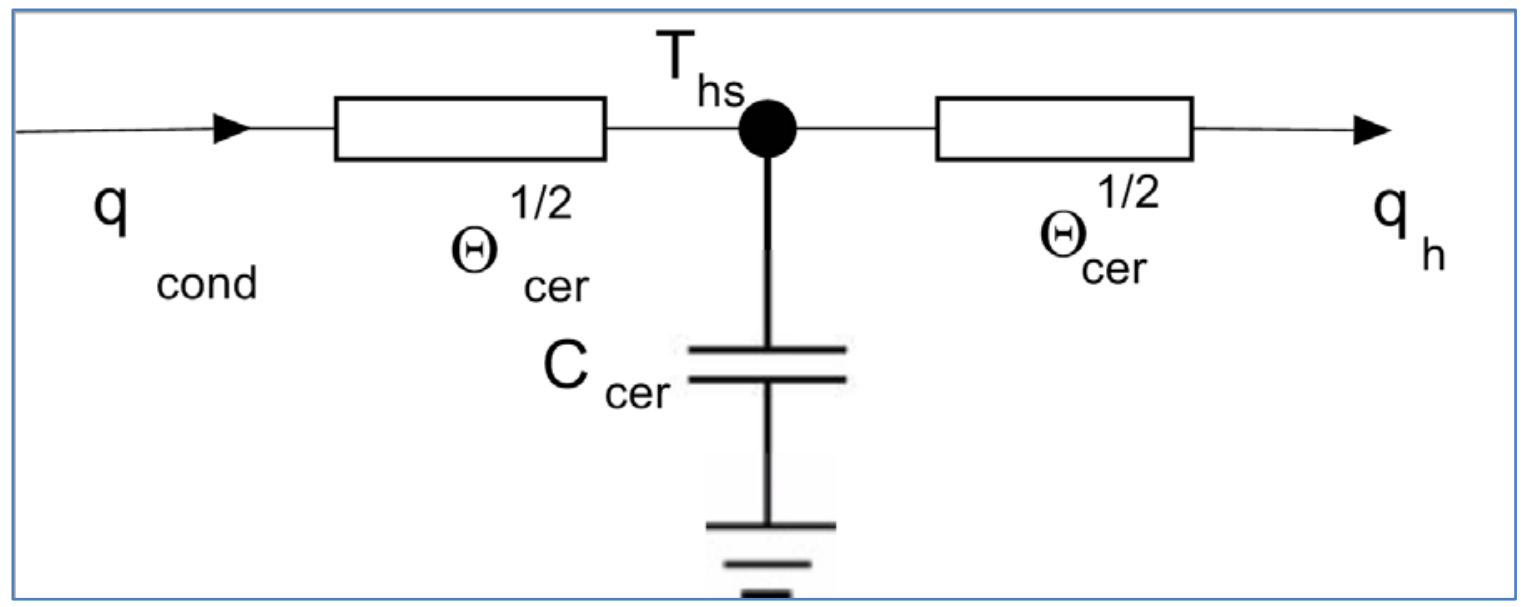

Figure. 6-4 Electrical circuit analogy of bottom ceramic plate

The conduction thermal resistance is given as:

$$
\Theta_{c e r}^{1 / 2}=\frac{L_{c e r}^{1 / 2}}{k_{c e r} A_{c e r}}
$$

where $L_{c e r}^{1 / 2}$ and Acer is the length of half part of the ceramic plate in the direction and area of TEG module respectively and are in the direction where the ceramic plate conducts heat to the TEG module containing the P-N pellets and conductors. The heat conducted to the TEG module is given as $q_{\mathrm{h}}$.

For a typical TEG module of area $40 \mathrm{~mm} \times 40 \mathrm{~mm} \times 0.77 \mathrm{~mm}$, the lateral area is much smaller than the area of the two ends, thus convection heat transfer from lateral sides is considered to be negligible. The heat capacity of the ceramic plate is given as $\mathrm{C}_{\text {cer. }} \mathrm{A}$ thermoelectric module consists of number of thermoelectric pairs connected in series by copper solder and contained between thermally 
conductive and electrically insulated ceramic plates. The simulation of hundreds of $\mathrm{P}-\mathrm{N}$ pairs and copper solders inside each module in an array would result in very high computational complexity. Thus, the elements are all aggregated into TEG cuboid and thermal properties for the aggregated cuboid could be derived. Chen and Snyder [81] described a method to extract key parameters from manufactures datasheets. They showed that the compact model has good accuracy as compared to three dimensional full resolutions.

The heat transfer inside the TEG modules at the hot side could be described as:

$$
q_{h}=I T_{h} \alpha_{m}+\frac{\left(T_{h s}-T_{c s}\right)}{\Theta_{T E G, T}}-\frac{R_{T E G}}{2} I^{2}
$$

And at the cold side of the modules:

$$
q_{c,}=I T_{c} \alpha_{m}+\frac{\left(T_{h s}-T_{c s}\right)}{\Theta_{T E G, j}}+\frac{R_{T E G}}{2} I^{2}
$$

where $\alpha_{m}$ is the Seebeck coefficient for a TEG module. The first terms in Eq.6.2.9 $\mathrm{IT}_{\mathrm{h}, \mathrm{jam}}$ and Eq. 6.2.10 IT $\mathrm{T}$,jam are the Peltier heat terms at the hot side and cold side of TEG module respectively. They are represented by $\mathrm{q}_{\mathrm{ph}}$ and $\mathrm{q}_{\mathrm{pc}}$ in Figure 6-5. 


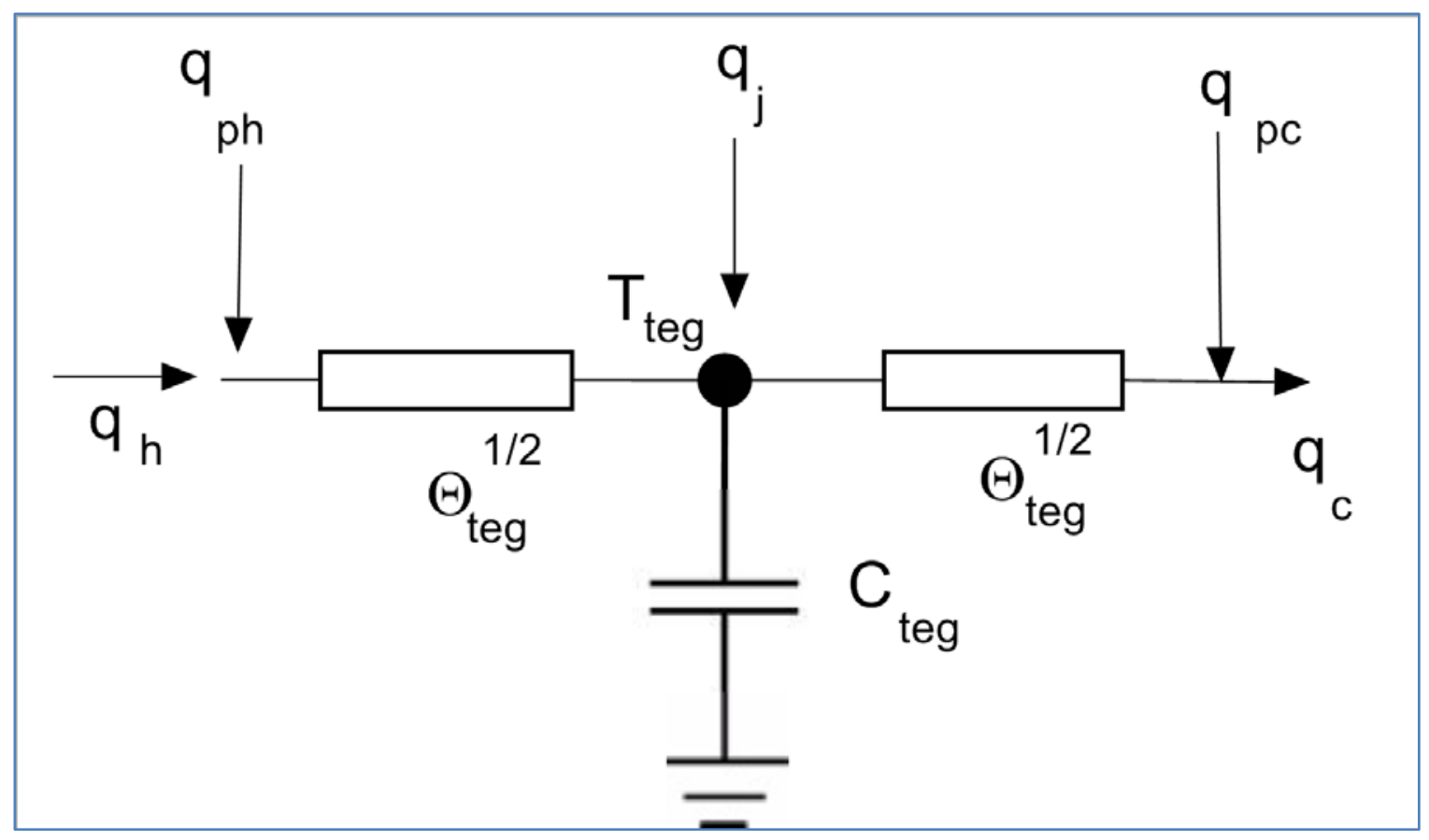

Figure. 6-5 Electrical circuit analogy of TEG module (P-N pellets and conductor)

Second term in both equations is the conduction heat terms and the last term represent Joule heating inside the modules which is indicated by $q_{j}$ in Figure 6-5. The manufactures data sheet provides the hot side ( $\left.T_{h s}\right)$ and cold side ( $\left.T_{c s}\right)$ temperature of the TEG module for a given $\mathrm{q}_{\mathrm{h}}$ and the resulting current $(\mathrm{I} L)$ when TEG module is connected to a load with matching electrical resistance.

The Seebeck coefficient can be determined using the relation:

$$
\alpha_{m}=\frac{V_{o c}}{T_{h s}-T_{c s}}
$$


Where the value of open circuit voltage for a given $T_{h s}$ and $T_{c s}$ is provided in the datasheets. Thus, the total conduction thermal resistance of TEG module including the ceramic plates could be found using the relation:

$$
\Theta_{T E G, T}=\frac{T_{h s}-T_{c s}}{q_{h}-\alpha_{m} I_{L} T_{h s}+\frac{I_{L}^{2} R_{e}}{2}}
$$

The total conduction thermal resistance consists of the top and bottom ceramic plates in addition to the thermoelectric cuboid containing P-N pellets and copper solder which are connected in series thermally. Thus, the thermal resistance of thermoelectric cuboid is given by:

$$
\Theta_{\text {teg }}=\Theta_{T E G, T}-2 * \Theta_{c e r}
$$

The equivalent conduction thermal coefficient kteg could also be defined as:

$$
k_{\text {teg }}=\frac{L_{\text {teg }}}{\Theta_{\text {teg }} A_{\text {teg }}}
$$

The conduction thermal resistance is given as:

$$
\Theta_{\text {tegr }}^{1 / 2}=\frac{L_{\text {teg }}^{1 / 2}}{k_{\text {tegr }} A_{\text {teg }}}
$$


Where $L_{t e g}^{1 / 2}$ and $A_{\text {teg }}$ is the length of half part of the ceramic plate in the direction and area of TEG module respectively and are in the direction where the ceramic plate conducts heat to the TEG module containing the P-N pellets and conductors. The heat conducted to the TEG module is given as $q_{\mathrm{h}}$.

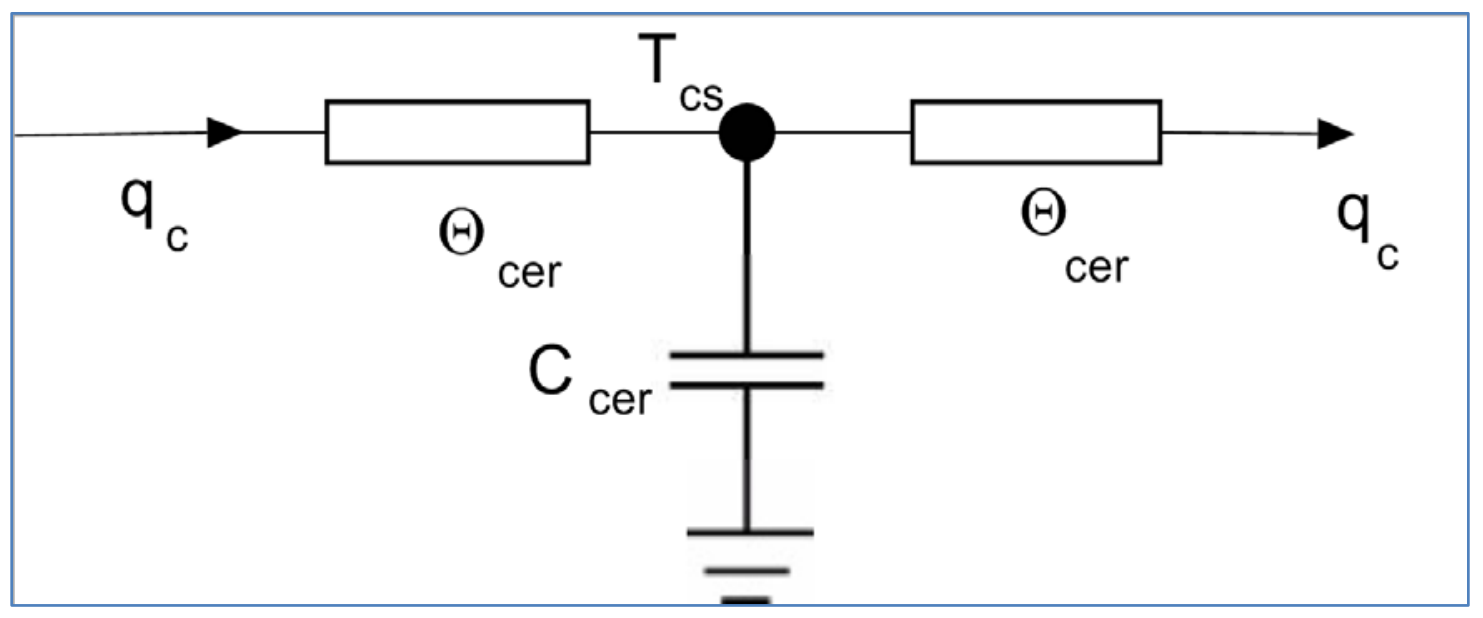

Figure. 6-6 Electrical circuit analogy of top ceramic plate

The top ceramic plate (Figure. 6-6) has an average temperature of $T_{c s}$ and conducts the heat to the cold heat sink. The equation and parameters are equivalent to the bottom ceramic plate.

\subsubsection{Cold side heat sink}

Cold side heat sink removes the heat from the cold side of the TEG module into the surrounding environment. It consists of number of fins (microchannels) in 
different of arrangement and geometries. It could also be represented by an equivalent conduction thermal resistance from base to tip $\left(\Theta_{x}\right)$ and convective thermal resistance of $\Theta_{\text {conv. }}$ The average temperature of the heat sink is given by Tx. The electrical circuit analogy for cold side heat sink is depicted in Figure. 6-7.

\subsubsection{Discretization of model}

The dynamic model is developed by discretizing the system in space and time. Each body is discretized into $\Omega$ parts. The value of $\Omega$ for each body could be made variable depending on the computational resources and the amount of resolution required in each body making the whole system. The volume of each discretized part could be calculated by:

$$
\Delta V_{x}=\frac{A L}{\Omega}
$$

Where $A$ is the frontal area on the heat path and $L$ is the total length of the body. 


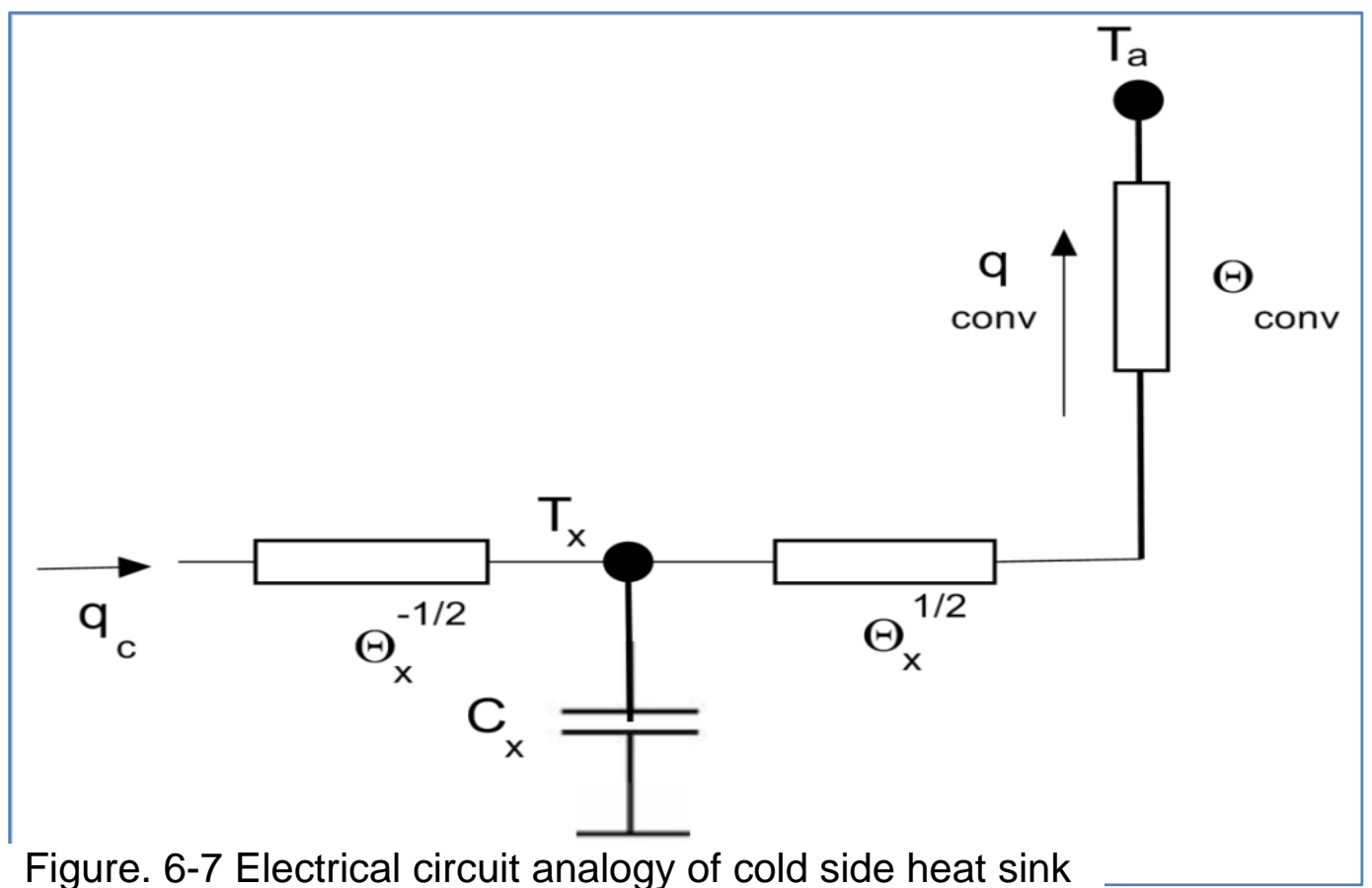

\subsubsection{Heat source device}

The heat source device (Figure. 6-8) is discretized into $\mathrm{n}$ equal parts with temperatures of $T(j)_{d}$ and thermal capacity $C(j)_{d}$ for $0 \leq j \leq n$. Each part has a pair of conduction thermal resistances, $\Theta(j)_{d}^{1 / 2}$ interacting with neighboring parts of the body. In addition, every part has convection thermal resistance, $\Theta(j)_{\text {conv }}$ to represent heat loss the surrounding atmosphere through lateral sides. For total length of $L_{d}$, each part has a length of $L_{d} / n$ and equal area of $A_{d}$. The body has heat input $\left(q_{i n}\right)$ and conducts heat $\left(q_{\text {cond }}\right)$ to the bottom ceramic plate side of TEG module. 


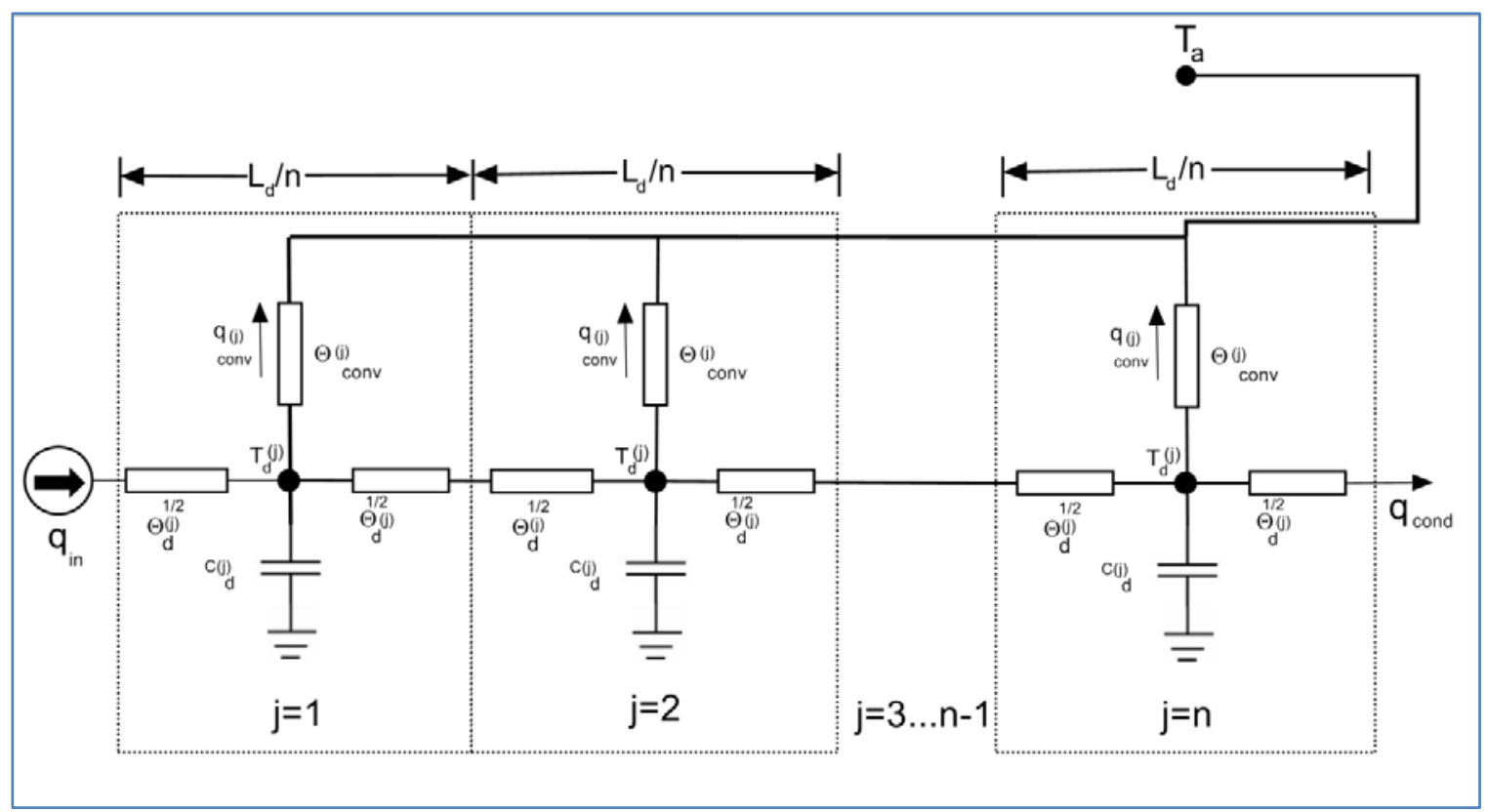

Figure. 6-8 Discretized form of heat source device

\subsubsection{Bottom ceramic plate of TEG module}

Heat is conducted ( $q_{\text {cond }}$ ) to the bottom ceramic plate from the heat source device. For the total ceramic length $L_{c e r}$, the body is divided into $L_{c e r} / n$ parts. Each part (

Figure. 6-9) has a temperature of $T(j)_{c e r}$ and the temperature of the part neighboring the device has a temperature of $\mathrm{T}(0)_{\mathrm{cer}}=\mathrm{T}_{\mathrm{hs}}$. 


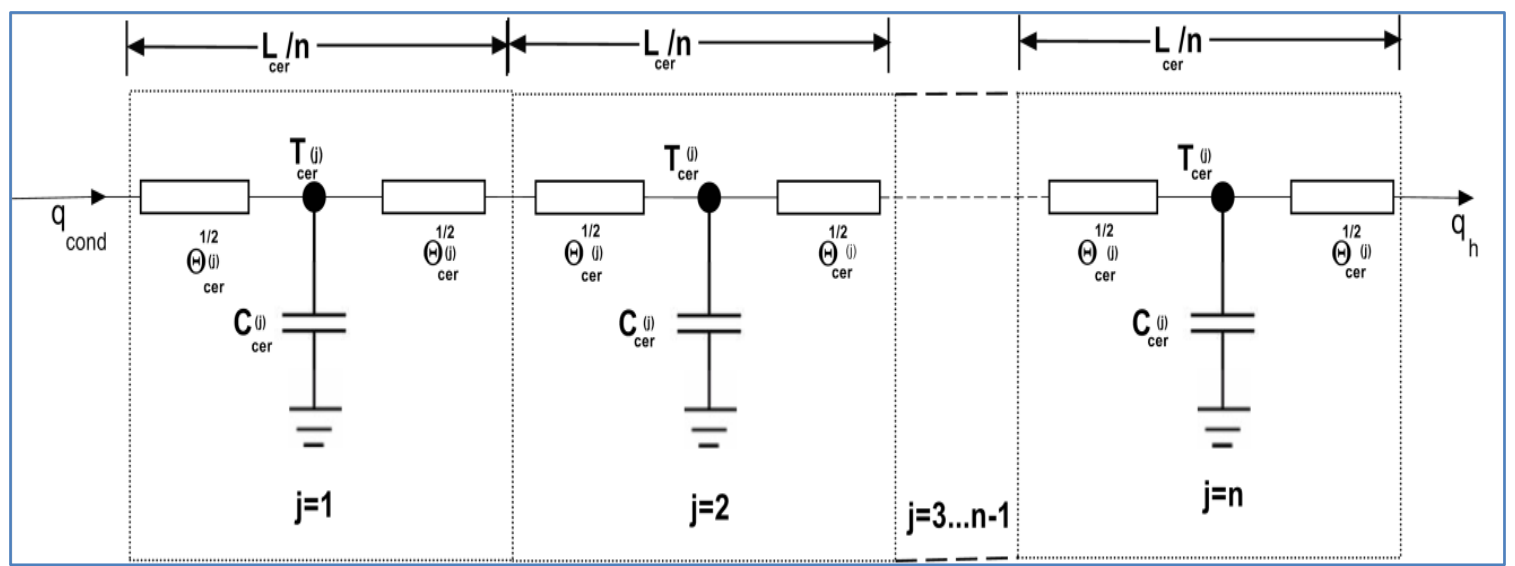

Figure. 6-9 Discretized form of bottom ceramic plate

\subsubsection{TEG cuboid (P-N pellets and conductor)}

The bottom side of ceramic plate conducts heat to the side of TEG cuboid representing P-N pellets and conductors. On the hot side of TEG cuboid (Figure. 6-10), a planar heat term of Peltier heat ( $\mathrm{qph}_{\mathrm{ph}}$ is applied. Each part of TEG cuboid has a temperature of $T(j)$ teg and a volumetric heat generation term of $q_{J} / n$ representing Joule heating.

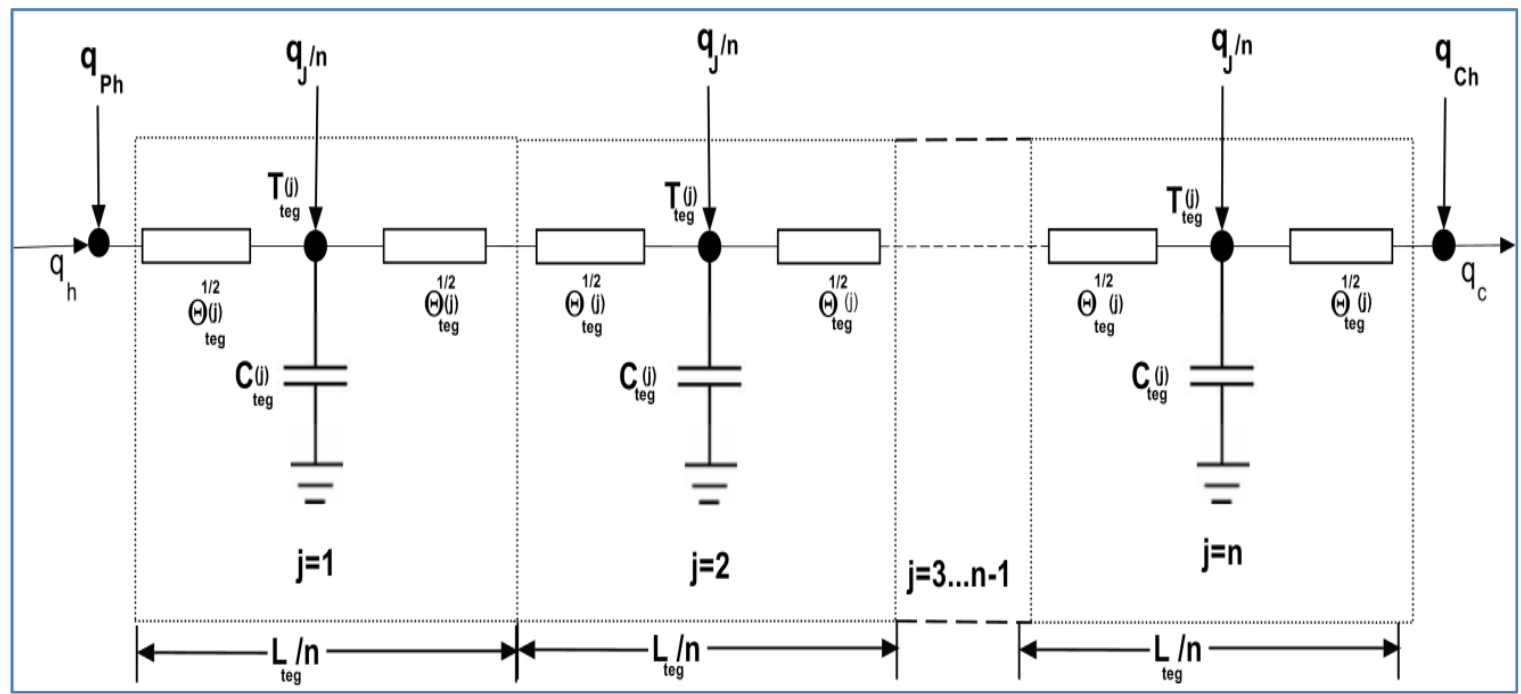


Figure. 6-10 Discretized form of TEG module (P-N pellets and conductor)

\subsubsection{Top ceramic plate of TEG module}

As Figure. 6-11 indicates, the discretization of top insulation plate of TEG module is equivalent to bottom plate. The TEG cuboid conducts heat into the top ceramic plate which in turn transmits the heat to the bottom side of the cold side heat sink. The temperature of the part neighboring the cold side heat sink has a temperature of $T(n)_{\text {cer }}=T_{\text {cs }}$.

\subsubsection{Cold side heat sink}

After the cold side heat sink is represented by equivalent body with conduction and convection terms, it could then be divided into $\mathrm{n}$ parts to account for temperature variation along the length of the heat sink (Figure. 6-12). The cold side heat sink convects the heat to the surrounding environment via convection heat transfer. The convection resistance term is represented by $q_{c o n v, x .}$ 


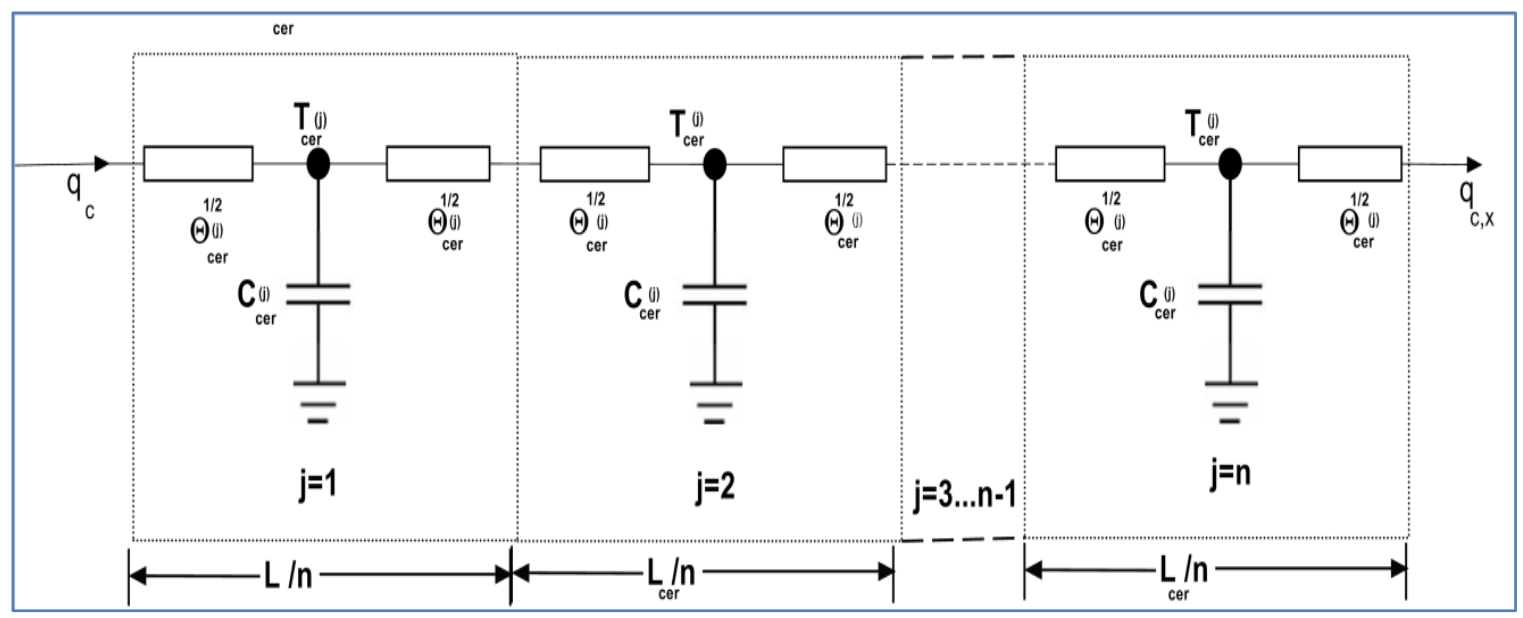

Figure. 6-11 Discretized form of top ceramic plate

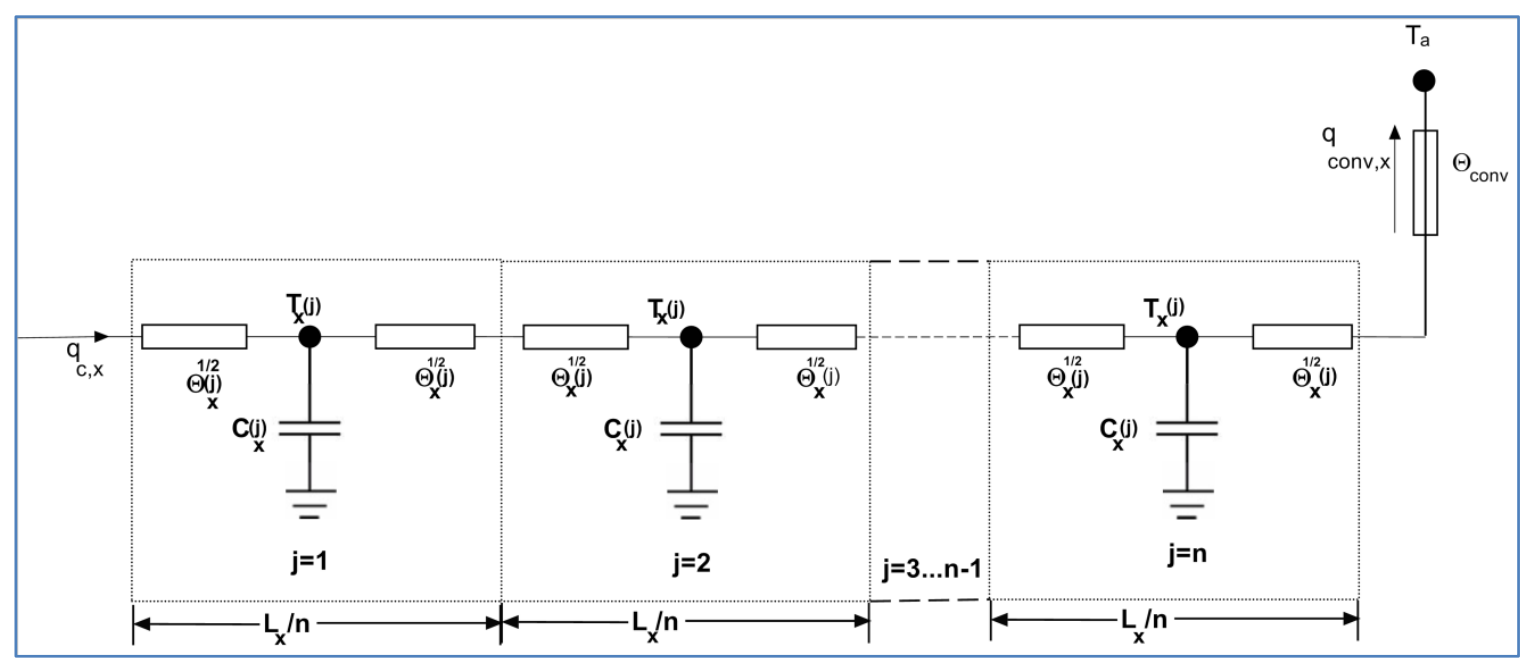

Figure. 6-12 Discretized form of cold side heat sink

\subsection{Modeling electrical system of TEG module}

The basic modeling of TEG module entails determining the hot and cold side temperature of TEG module as inputs to the model (Figure. 6-13). Thus the TEG electrical model is coupled with the thermal model to derive temperature of the TEG module at every time step/iteration. The electrical model has voltage and 
current as an output. The heat terms (Peltier, Joule and Thomson) are functions of current which calls for the electrical output to be coupled with the thermal model.

For an array of TEG modules connected in series to a load, total voltage at any given instant t, VT (t) and the current in the array, I(t) are given as:

$$
V_{T}(t)=\sum_{j=1}^{N_{m}}\left(\alpha_{m}(t)\left(T_{h s}(t)-T_{c s}(t)\right)-\left(N_{m} R(t)_{e, T E G}+R_{e, l d}+R_{e, c}\right) I(t)\right.
$$

$$
I(t)=\frac{\sum_{j=1}^{N_{m}} \alpha_{m}(t)\left(T_{h s}(t)-T_{c s}(t)\right)}{N_{m} R(t)_{e, T E G}+R_{e, l d}+R_{e, c}}
$$

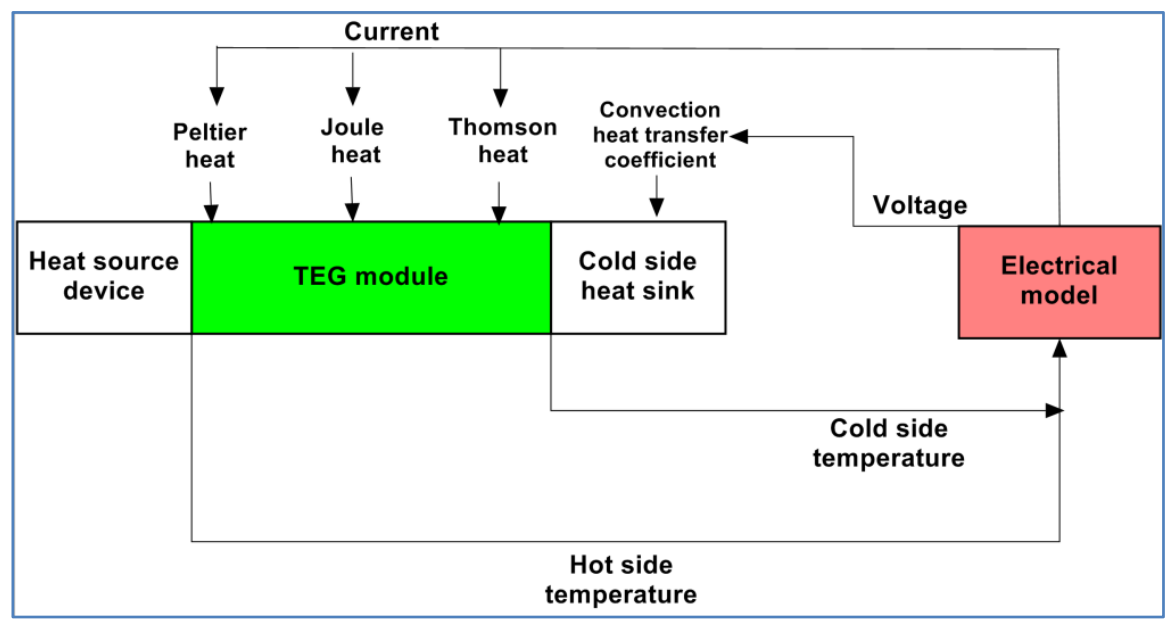

Figure. 6-13Coupling of electrical and thermal model 
where $\mathrm{Nm}$ is the number of modules in an array and $\alpha(t)$ and $R(t) e$,TEG are the Seebeck coefficient and electrical resistance of TEG module at an instant $t$ respectively. Different methodologies can be employed to determine the properties of TEG module like Seebeck coefficient, thermal and electrical resistance.

The first method to determine the properties of the module is by aggregating the properties of P-N legs.

The Seebeck coefficient for a module ( $a \mathrm{~m}$ ) could be calculated as:

$$
\alpha_{m}=n_{p, n}\left(\alpha_{p}-\alpha_{n}\right)
$$

where $n_{p, n}$ is the number of thermoelectric pairs in the module and ap and an are the Seebeck coefficient of the P-doped and N-doped legs respectively.

The thermal resistance for thermoelectric P-type leg $\left(\Theta_{\mathrm{th}, \mathrm{P}}\right)$ and $\mathrm{N}$-type leg $\left(\Theta_{\mathrm{th}, \mathrm{n}}\right)$ is given as:

$$
\begin{gathered}
\Theta_{t h, p}=\frac{L_{p}}{A_{p} k_{p}} \\
\Theta_{t h, n}=\frac{L_{n}}{A_{n} k_{n}}
\end{gathered}
$$


Where Lp, Ln, Ap, An, kp, kn are the lengths, cross-sectional areas and thermal conductivity of the P-type and N-type legs respectively.

As a module consists of the legs connected in parallel thermally, the total electrical resistance of a module from the legs is given as:

$$
\Theta_{t h, p n}=\left[n_{p, n}\left(\frac{A_{p} k_{p}}{L_{p}}+\frac{A_{n} k_{n}}{L_{n}}\right)\right]^{-1}
$$

As there are also air gaps between the legs, the thermal resistance from air gaps can be approximated as:

$$
\Theta_{t h, a}=\frac{L_{a, a v}}{A_{a} k_{a}}
$$

Where $L_{a, a v}$ and $k_{a}$ are the average length of air gap and thermal conductivity of air and the area of the total air gap in a module Aa can be expressed as:

$$
A_{a}=A_{m}-n_{p, n}\left(A_{p}+A_{n}\right)
$$

where Am is the total area of TEG module and 


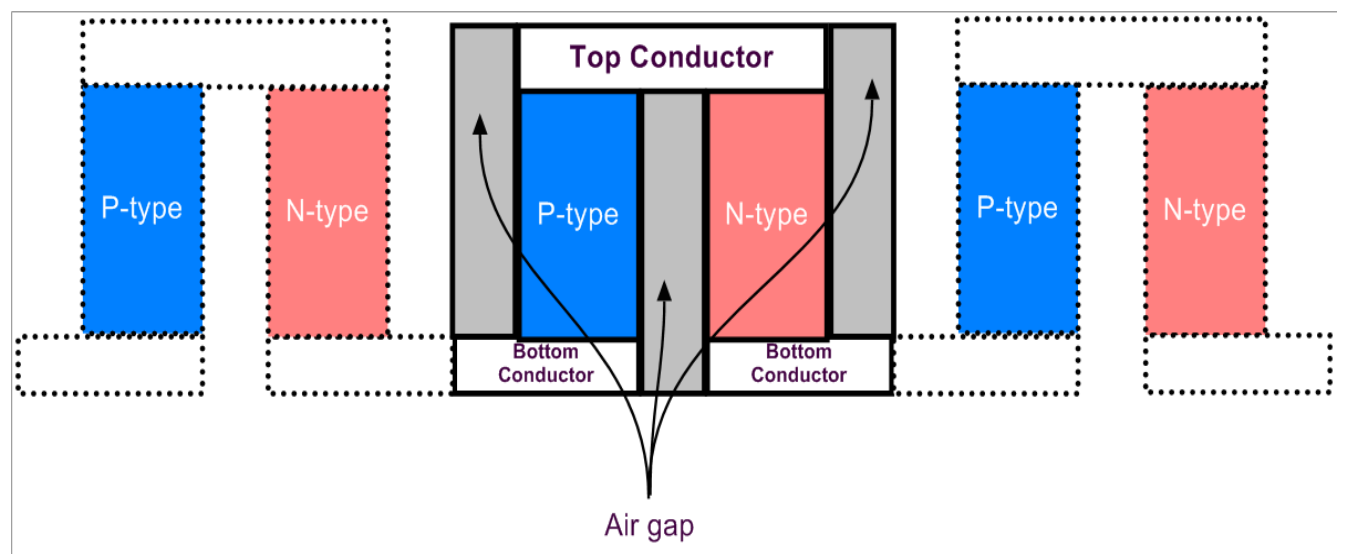

Figure. 6-14 $\mathrm{P}$ and $\mathrm{N}$ type legs with air gap

As the P-Type and N-type elements and the air gap are connected in parallel thermally (Figure. 6-14), the total thermal resistance for a TEG module ( $\left.\Theta_{\mathrm{th}, \mathrm{TEG}}\right)$ can be calculated as:

$$
\Theta_{t h, T E G}=\left[\frac{1}{R_{t h, p n}}+\frac{1}{R_{t h, a}}\right]^{-1}
$$

The electrical resistance for thermoelectric P-type leg $\left(R_{e l, P}\right)$ and $N$-type leg $\left(R_{e l, n}\right)$ is given as:

$$
R_{e l, p}=\frac{L_{p}}{A_{p} \sigma_{p}}
$$




$$
R_{t h, n}=\frac{L_{n}}{A_{n} \sigma_{n}}
$$

where $L p, L n, A p, A n, \sigma_{p}, \sigma_{n}$ are the lengths, cross-sectional areas and electrical conductivity of the P-type and N-type legs respectively.

For the copper conductors, the electrical resistance is given as:

Top conductor:

$$
R_{e l, c u_{-} T}=\frac{L_{c u_{-} T}}{A_{c u_{-} T} \sigma_{c u}}
$$

Bottom conductors (pair of bottom legs):

$$
R_{e l, c u_{-} B}=\frac{2 * L_{c u_{-} B}}{A_{c u_{-} B} \sigma_{c u}}
$$

As the $\mathrm{P}$ and $\mathrm{N}$-type legs and conductor are connected in series electrically, the total electrical resistance for a module Rel,TEG is given as:

$$
R_{e l, T E G}=n_{p, n}\left(R_{e l, p}+R_{e l, n}+R_{e l, c u_{-} T}+R_{e l, c u_{-} B}\right) \quad 6.3 .14
$$

Second method is deriving the parameters from manufactures data sheet. TEG module manufactures provide some data that described the performance of the modules. It basically involves the hot side (Ths) and cold side temperature (Tcs) 
and open circuit voltage (Voc) for the test conditions. Thus, the Seebeck coefficient can be found using:

$$
\alpha_{m}=\frac{V_{o c}}{T_{h s}-T_{c s}}
$$

The manufactures also provided the current at matched load $(\mathrm{I} L)$ and the heat input at the hot side of TEG module $\left(q_{h}\right)$. Thus, total thermal resistance for TEG module, Rth,TEG can be determined as:

$$
\Theta_{t h}=\frac{T_{h s}-T_{c s}}{q_{h}-\alpha_{m} I_{L} T_{h s}+0.5 I_{L}^{2} R_{e l, T E G}}
$$

The electrical resistance $R_{e l, T E G}$ is provided in the datasheet.

\subsection{Dynamic model implementation in MATLAB/Simulink}

The dynamic model is constructed with Simscape (incorporated as a toolbox in Simulink) which is a physical modeling tool by Mathworks[95]. In Simscape, physical components and relationships are constructed using blocks. The equations representing the block/system are solved. 
A Simscape model also constitutes connections between blocks to define the relation between the models. The connections are bi-directional allowing the flow of signal in both directions.

Heat transfer modes are defined in terms of thermal elements which represent conduction, convection and radiation heat modes. The conduction block represents a simplification of the differential form of Fourier's Law of thermal conduction which is given as:

$$
\vec{q}_{\text {cond }}=-k \nabla T
$$

where $\vec{q}$ is heat flux density and $\nabla T$ is the temperature gradient.

The equation can be integrated over a heat conduction surface to get:

$$
\frac{\partial Q}{\partial t}=-k \int_{A} \nabla T \cdot d A
$$

In Simscape, the integrated form of Eq. 6.4.2 is used in one dimensional form and for a homogenous material between two points with temperature difference of $\Delta T$ :

$$
\frac{\Delta Q}{\Delta t}=-k A \frac{\Delta T}{\Delta x}
$$


where $\Delta x$ is the distance between the two points.

The convective block represents convection heat transfer as described by Newton's law of cooling which describes convection heat transfer between two bodies:

$$
Q_{\text {conv }}=h A\left(T_{A}-T_{B}\right)
$$

where $\mathrm{h}$ represents the convection heat transfer coefficient.

A Simscape language is utilized to create bodies (components), define nodes, variables, parameters and equations describing the system. The components have inlet/outlet ports to send and receive variables or signals. The first kinds of ports are termed as physical conserving ports which carry variables. They have a bidirectional connection and carry same value if directly connected. The second types of ports are physical signal ports and transmit physical signal between the components.

The sample workspace for the Simscape modeling a TEG based self-cooling system is represented on Figure. 6-15. The main subsystems are the thermal model and electrical model. 


\subsubsection{Thermal model}

The thermal system consists of a heat source device, TEG model and cold side heat sink model. The device has been segmented in 5 parts (Figure. 6-16a) to increase the accuracy of the models. As the number of parts represented by a single temperature and heat capacity is increased, the accuracy of the model is also augmented. The decision on the number of parts to use depends on the analysis of the Biot number.

Each part has an inlet port to receive heat from adjacent part and outlet port to conduct heat out of the part. It has also connection to convectively lose heat from lateral sides. The detail of each part is again shown in Figure. 6-16b. The TEG module is represented in Figure. 6-17. It is segmented in to number of parts. Each part has an inlet and outlet port for heat conduction. 


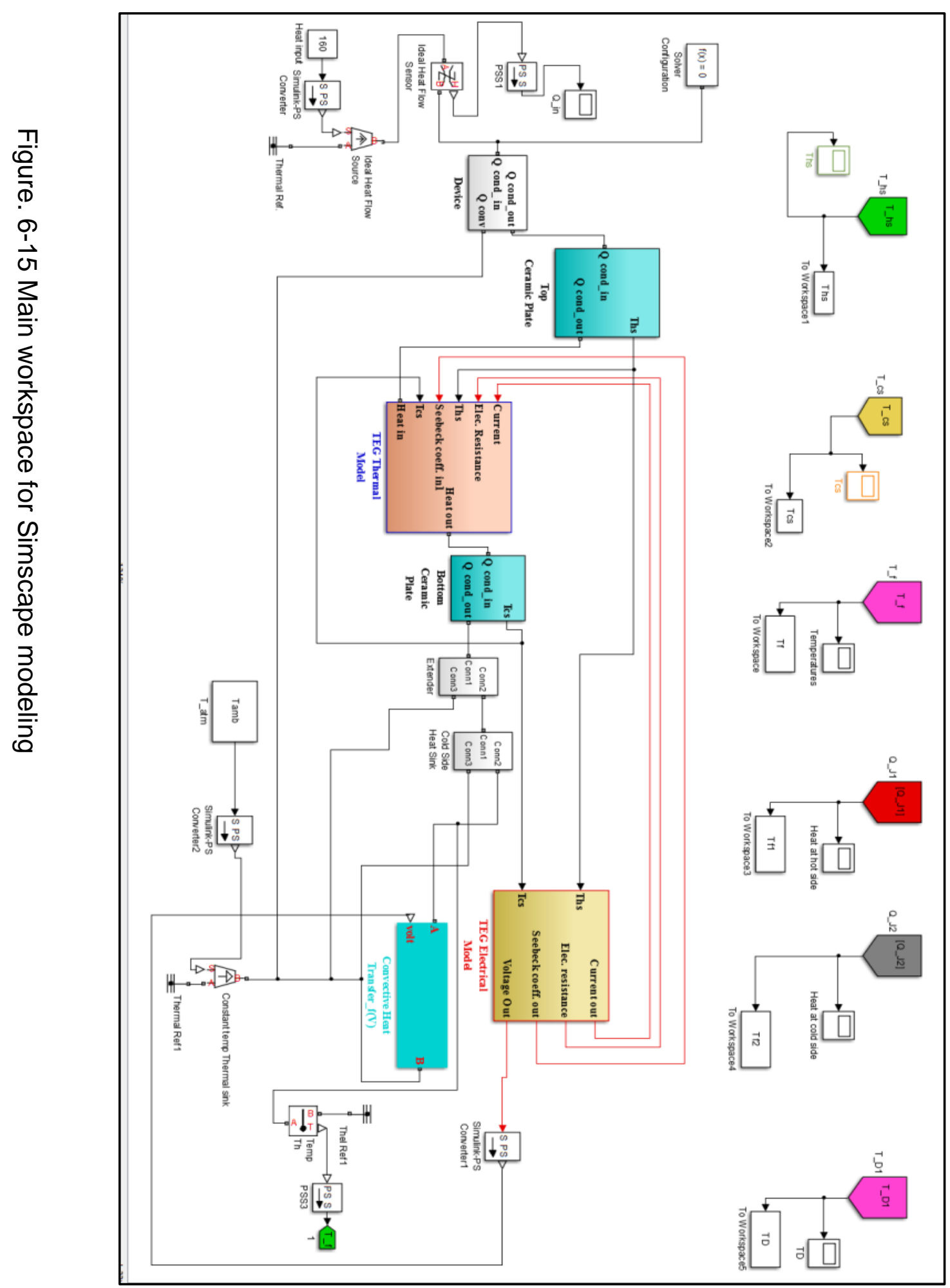


Heat transferred by conduction from adjacent part. In addition, volumetric heat generation is included in each part. A joule heat function receives current and electrical resistance the TEG electrical model and calculates joule heat which then fed into each part. Thomson heat also could be similarly defined and included in the model.

Peltier heat terms are included on either end of TEG model. Peltier functions receive the hot side and cold side temperatures from the thermal model as well as the value of current and Seebeck coefficient from the electrical model.

\subsubsection{Electrical model}

The electrical model in Figure. 6-18 consists of components that represent voltage, current and power output from TEG modules. The values of current at each time step is coupled with the thermal model to calculate the Joule, Peltier and Thomson heat terms.

\subsubsection{Coupling of Cold side heat sink with Electrical model}

For self-cooling system, it is necessary to couple the cold heat sink with the electrical model. This is done by defining a convection heat transfer block with receives voltage signal from electrical system. As the relation between fan input 
voltage and convection heat transfer can be defined as shown in previous section, it is possible to enter this relation using Simscape language.
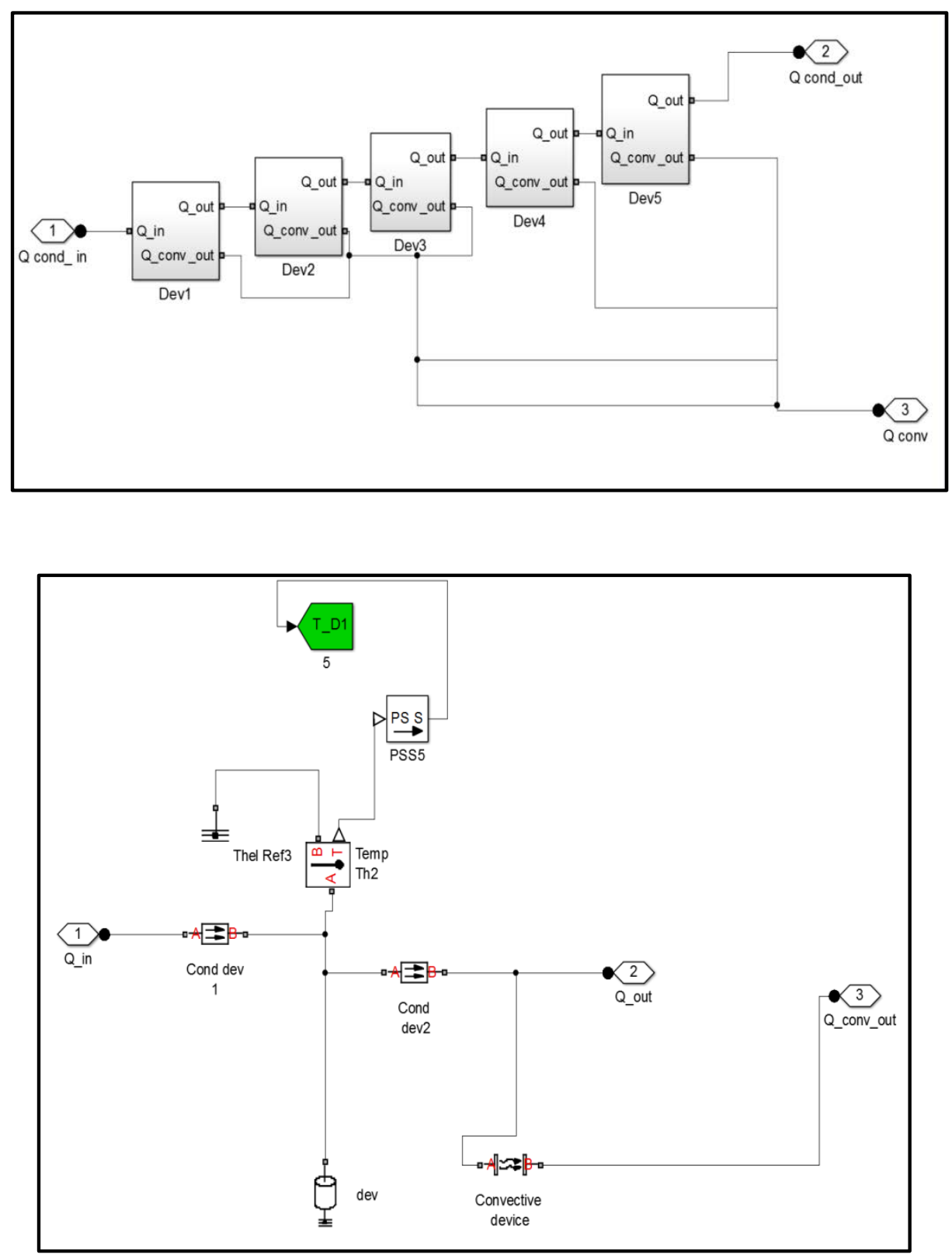

Figure. 6-16 a) Device subsystem divided in to 5 subsystems (Dev1-Dev 5) b) Details of one of the parts of the device (Dev1) 


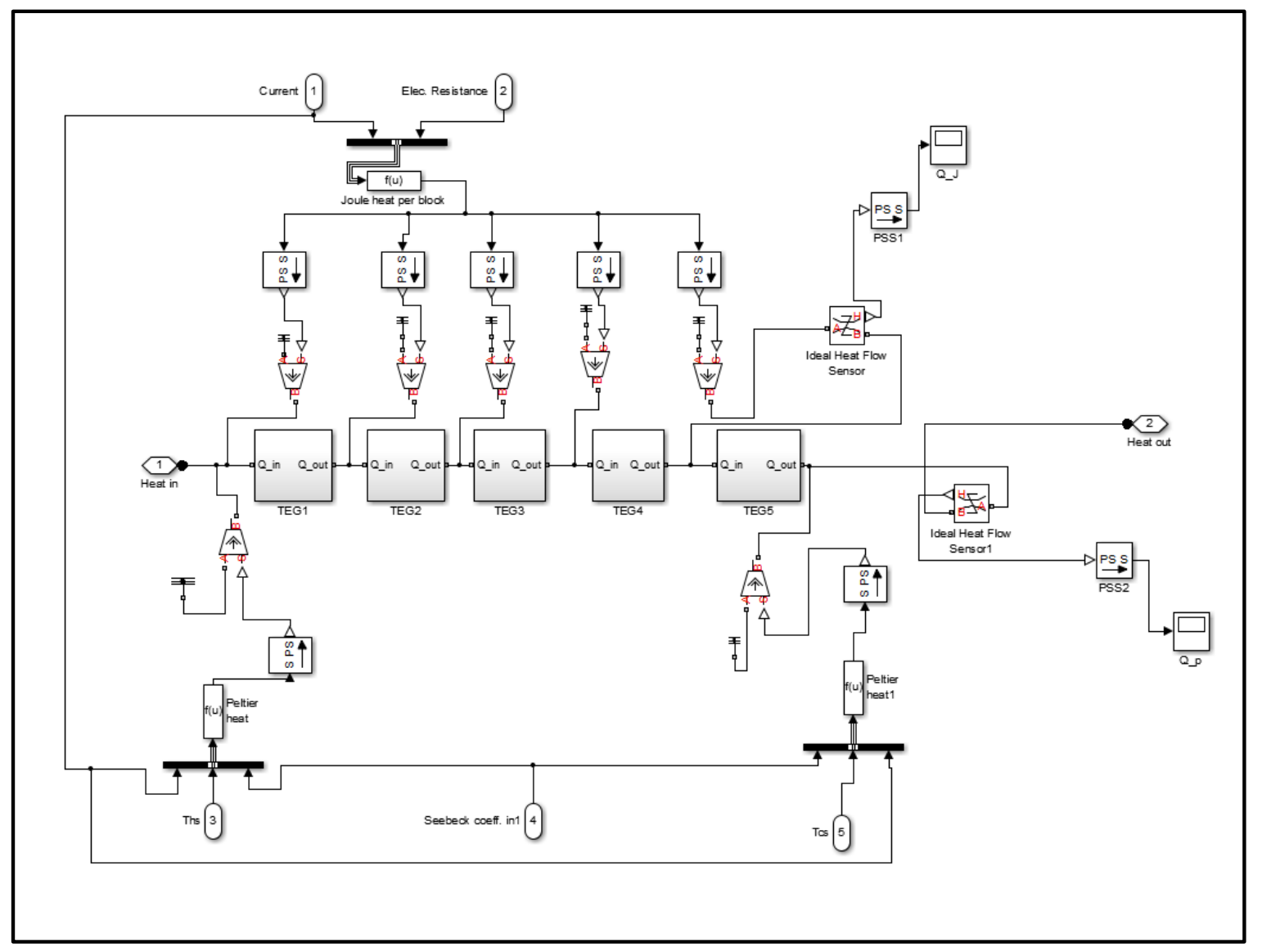

Figure. 6-17 TEG thermal subsystem

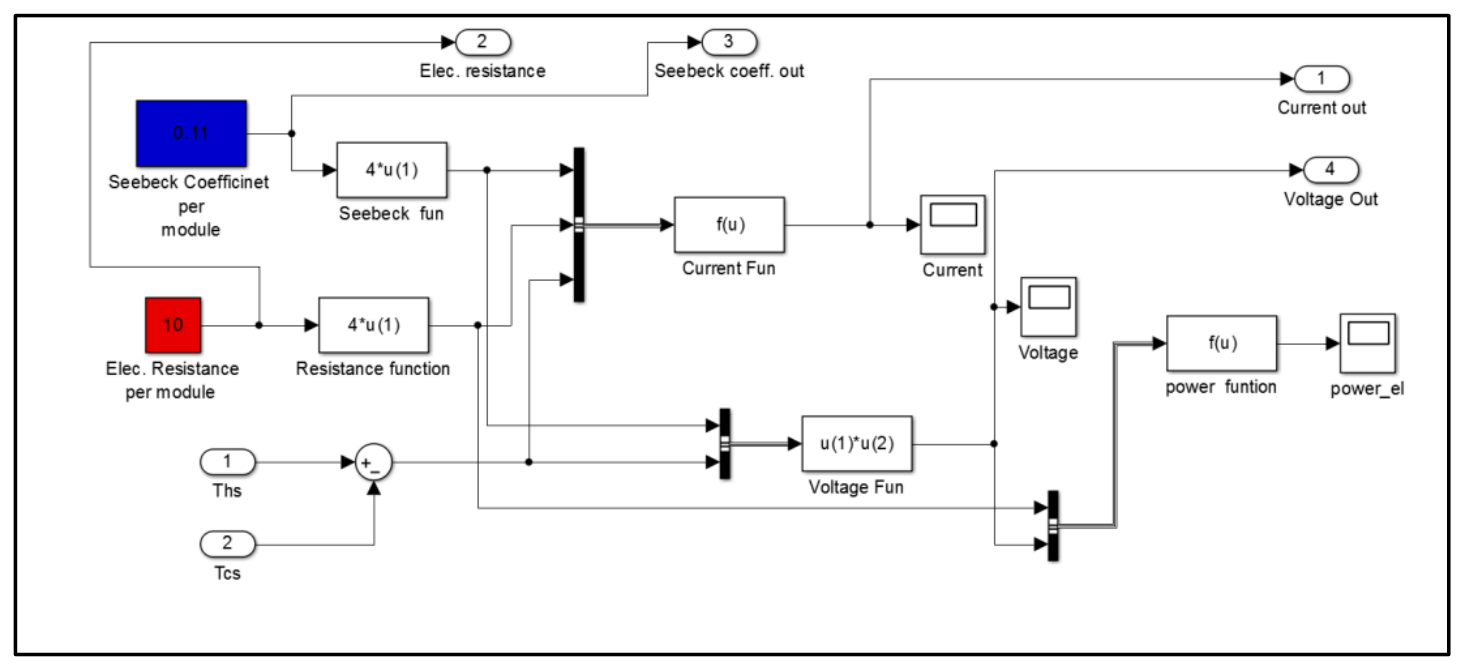

Figure. 6-18 Electrical model 


\subsection{Result and Discussion}

Thus, the Simscape model is implemented in MATLAB R2013a Simscape[95]. A personal computer with Intel $₫$ Xenon $\circledast$ CPU processor with speed of $2.40 \mathrm{GHz}$ is used for the simulation. The running time for the most of the simulation has been in order of few seconds. The results of the model is validated by comparing it using experimental data from [78]. The temperature of the device with respect to the surrounding environment $\left(\Delta T_{d e}\right)$ for transient heating for 160 minutes is compared against the experimental data in Figure. 6-19. The dynamic model has been able represent the data to a good accuracy. The running time was few seconds which make the dynamic modeling a fast and accurate method to simulate how the system dynamically performs.

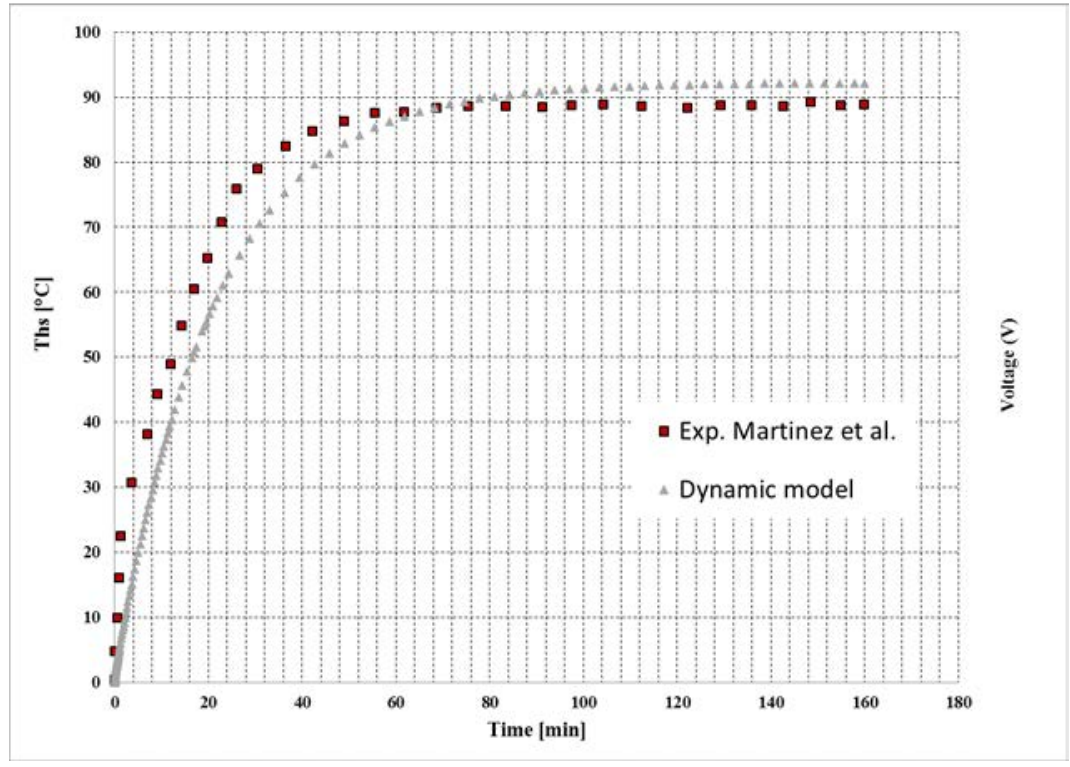

Figure. 6-19 Comparison of Dynamic model result for $\Delta T_{\text {de }}\left(T_{d}-T_{\text {env }}\right)$ with experimental data [3] 
The dynamic model has also been validated by comparison to our experiments results which have been described in detail in previous chapters. It can be inferred from Figure. 6-20 that the model has only slightly overestimated the temperature in case B for heat input of $120 \mathrm{~W}$. The average error was less than 3\%. Similarly, for case A, the model is compared (Figure. 6-21) against experimental data for $\mathrm{Q}_{\mathrm{in}}=80 \mathrm{~W}$ and the model has been able to predict the transient temperature variation with time with good accuracy (within 3.5\% error).

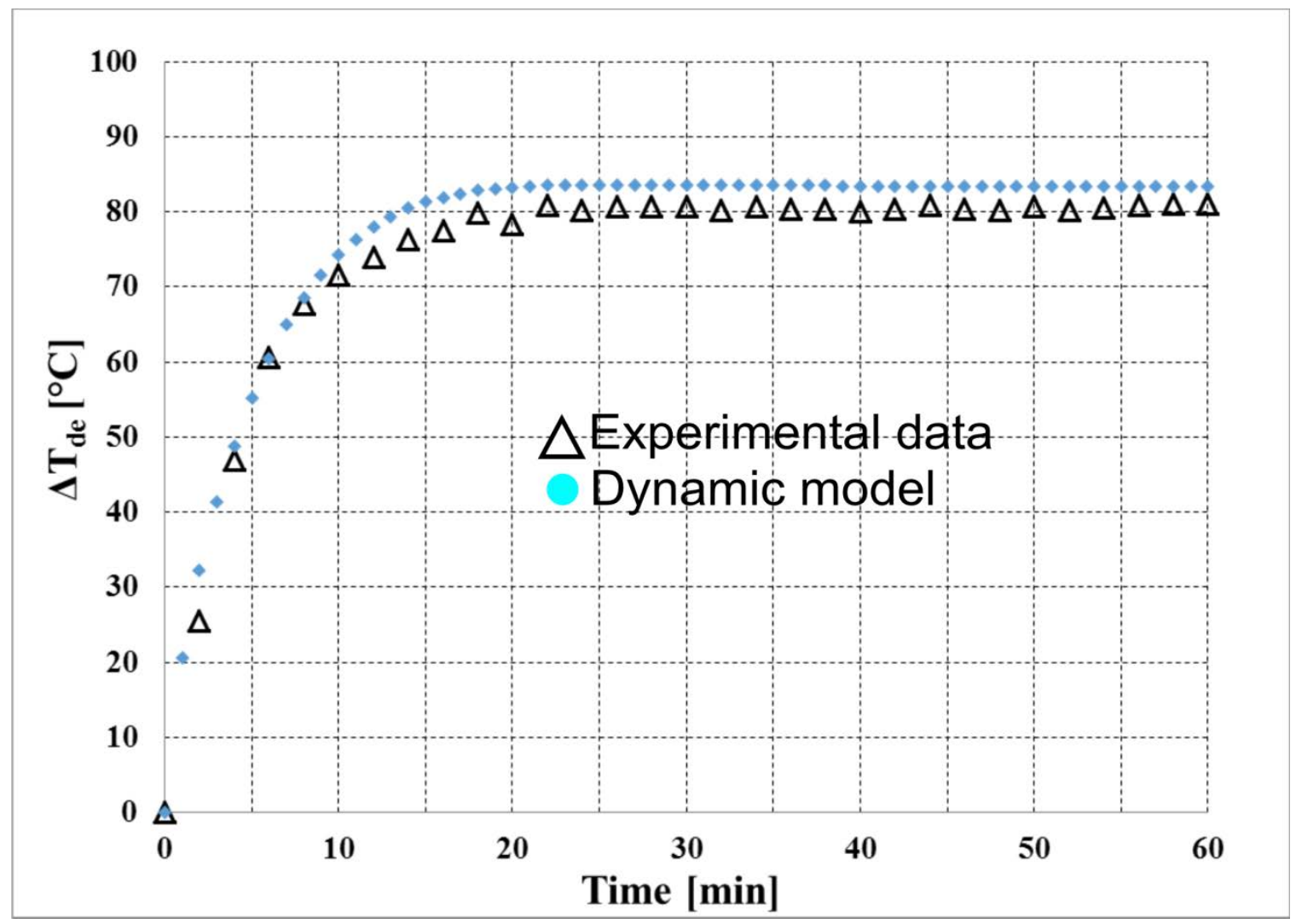

Figure. 6-20 Comparison of Dynamic model result for $\Delta T_{\text {de }}$ ( $\left.T_{d-}-T_{\text {env }}\right)$ with experimental data for Case $B$ and $Q_{\text {in }}=120$ 


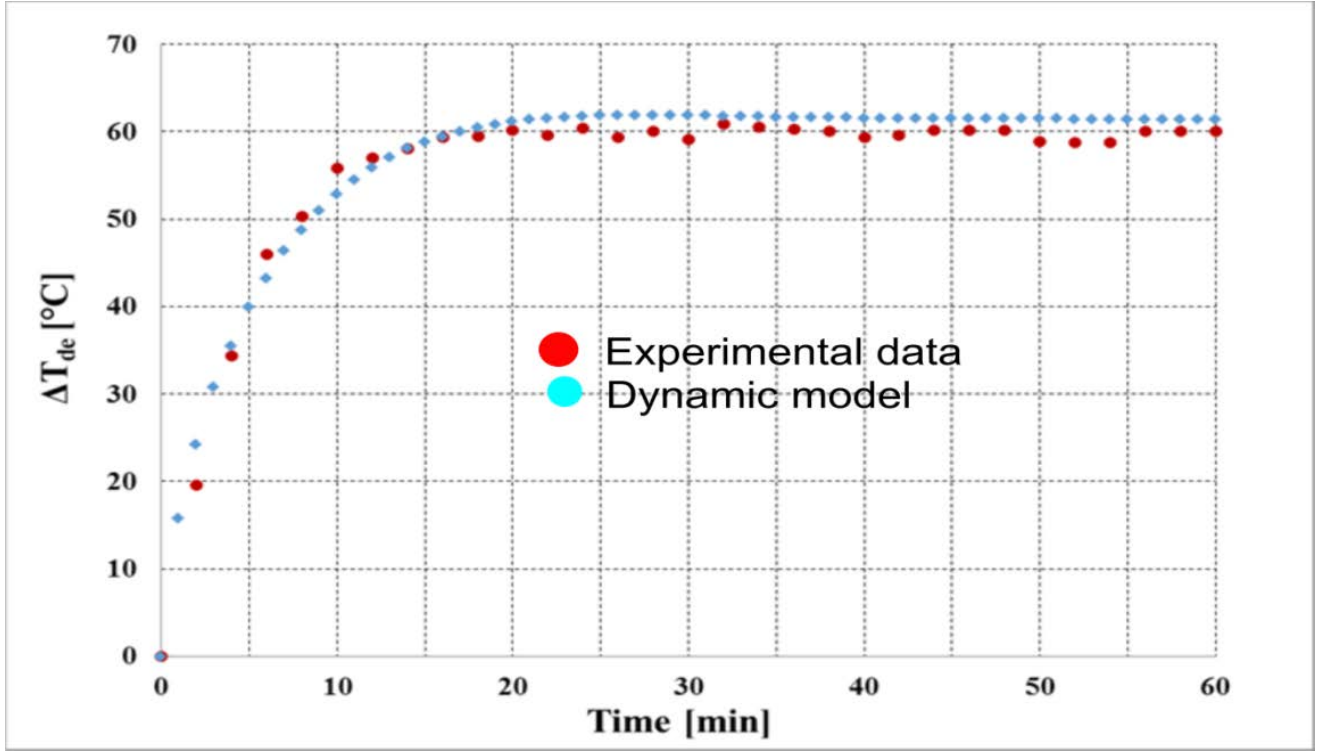

Figure. 6-21 Comparison of Dynamic model result for $\Delta T_{\text {de }}$ ( $T_{d}-T_{\text {env }}$ ) with experimental data for Case $A$ and $Q_{i n}=80 \mathrm{~W}$

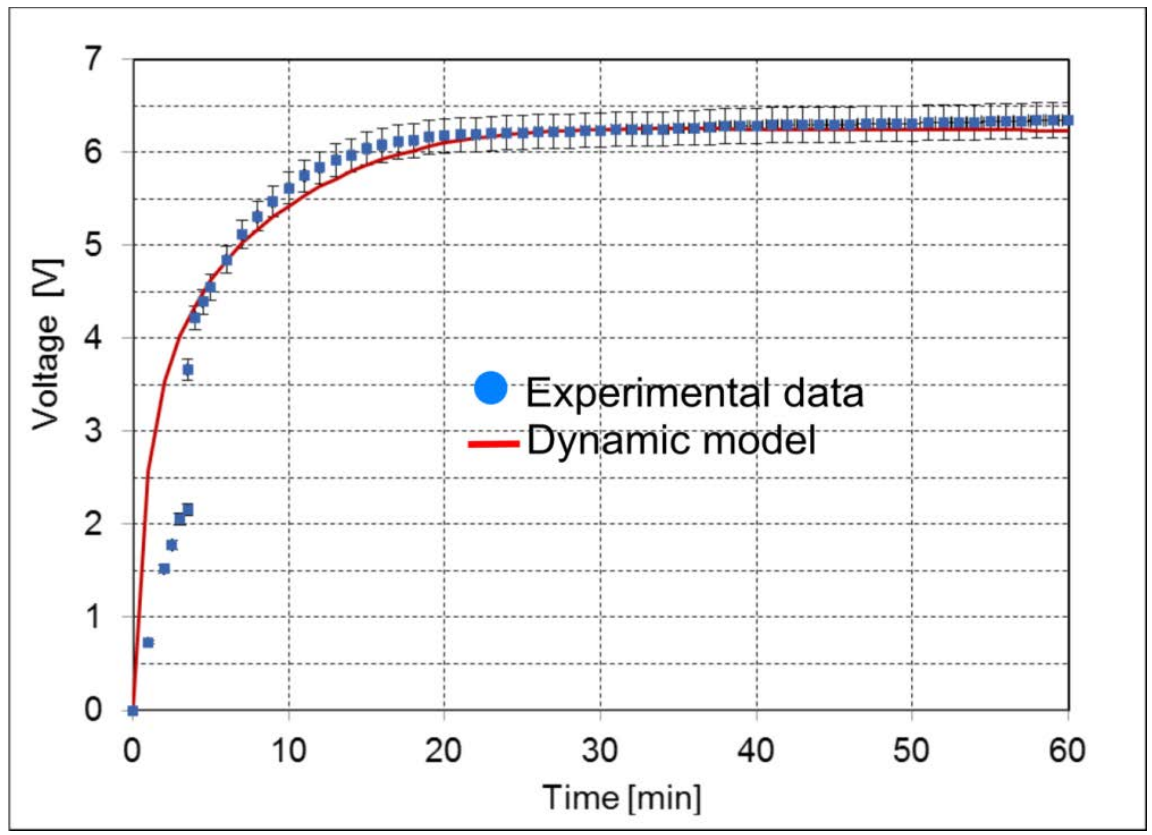

Figure 6-22 Transient variation of voltage for Case A in Qin=80 W 
The comparison of modeled transient variation of voltage produced by TEG with experimental data is shown in Figure 6-22. The model has captured the variation of voltage with time within an error of less than $3 \%$.

Thus, it has been shown that the model has the capability to capture the dynamic performance of a self-cooling device to a good accuracy with minimal computational resources 


\section{CONCLUSION}

In section 7.1 , summary of the dissertation is discussed which is flowed by suggestions for future studies in section 7.2.

\subsection{Summary of Dissertation}

In this dissertation, a full analysis tool for self-cooling system is developed using first principles of thermodynamics. Starting from first law of thermodynamics and second law of thermodynamics, energy and entropy equation for the smallest unit of thermoelectric generator unit (thermoelectric couples) are developed. The analysis is extended to TEG module consisting of multiple thermoelectric couples. Multiple TEG modules are also connected in series electrically and in parallel thermally to form an array of TEG modules.

The basic models are developed with increasing resolution in terms of the general thermodynamic model analysis. The first model assumes a Carnot engine, which is followed by endoreversible heat engine. Finally, an irreversible real engine model which contains TEG module as a heat engine is studied. The three tier study provides an important insight into comparison of TEG module based heat engine with theoretical general heat engine models. The irreversible heat engine has been coupled with the load (fluid mover) to study power consumption at the load and provide insights to self-cooling potential. The 
effects of varying cold side heat exchange and size of heat engine are studied. A comprehensive tool which helps identify the range of operation points for selfcooling is also developed.

Important conclusions from the study are:

- Carnot heat engine and endoreversible heat engine models provide a general insights into the maximum possible heat transfer and power and temperature at the surfaces of heat engine. However, both models have poor correlation with an irreversible TEG based heat engine due to the inherent low heat to power potential of TEG module and internal irreversibility.

- More heat is transferred in the array due to lower thermal resistance resulting in lower hot side temperature of TEG module. The average temperature difference across the TEG array is however reduced due to lower thermal resistance. The open circuit voltage increases due to an increase in the total number of thermoelectric couples in the array, however the total value is less than the combined effect if each module was operated separately. The power output only increases slightly due to an increase in open circuit voltage but also there is also a counter effect of decreasing current which lessens the magnitude of increase. 
- For certain configuration of cold-side heat exchange of self-cooling system, there is a maximum value of volume of fluid flow inside the fins of the heat exchanger which produces zero net power. If the volume flow rate is increased beyond this point, the system produces less power than it consumes, thus self-cooling is not possible.

- The other limiting factor is the system operation points at different voltages. For self-cooling to be viable, the voltage provided by TEG modules to the fluid mover should be able to correspond to the system operation points at a particular voltage.

- The voltage provide by the TEG modules should also be more than the starting voltage of the fluid mover. Thus, a "self-cooling window" could be defined to identify system operation points for a particular configuration of self-cooling system which provide a viable autonomous self-cooling system.

- The analysis tool could be used as an important early design tool and has been coded with Python to facilitate the ease of use. It could be used for parametric study and as well as an initial setup for optimization study.

Experiments have also been conducted to study the application of thermoelectric generators based self-cooling of devices. It has been shown that 
a self-sustaining cooling system could be achieved by scavenging the heat from the device to run a cooling system via a fan in parallel plate-fin cold heat sink.

Within the investigated operating ranges, it is found that:

- The base line model (case A) has provided forced convection cooling after generating electrical power that is a capable of running a fan. It has been able to reduce the device temperature by as much as $20 \%$.

- But Case A performs relatively poorly as compared to natural convection (case C) when the fan is not moving due to the presence of TEG modules in the primary heat path.

- By substituting part of TEG modules with a conductor in self-cooling case B, a $40 \%$ reduction in device temperature has been achieved as compared to natural convection. Moreover, the system, unlike case $A$, has similar performance with natural convection case C even when the fan is not moving.

- Thermal resistance analysis has shown convection thermal resistance plays the major part in case B and improvement in the design of cold-side heat sink is expected to further develop the performance of self-cooling.

In summary, self-cooling has been shown to have a potential to replace the use of external power sources to cool heat generating devices. As increased temperature difference results in increased cooling up to the maximum capacity 
of the fan, heat driven self-cooling has a capability to scale up automatically with increased heat input.

In this study, a general numerical methodology for the study of TEG based selfcooling is also developed. The methodology has three major parts. The fluid flow domain which is used to the study the effect of a variable speed fan and on the heat transfers and derive a relation of convection heat transfer coefficient with input voltage. The temperature and electric field variation is then studied using a coupled approach using an input from the fluid domain as boundary condition.

Experiments have been conducted on two types of self-cooling arrangements to measure the device temperature, voltage and power produced by TEG modules. It has been shown that the computational model is able to predict the experimental data with good accuracy (within 5\% error). A parametric study on the effect of electrical resistance of load $\left(R_{L}\right)$, height of fin $\left(H_{\text {fin }}\right)$ and fin density $\left(\mathrm{n}_{\text {fin }}\right)$ has been made. The power for self-cooling could be maximized by proper matching of number of TEG modules to the fluid mover (fan or pump) in terms of electrical resistance. An increase in $\mathrm{H}_{\text {fin }}$ decreases the fan power consumption and thermal resistance. It has also been shown that although an increase in $\mathrm{n}_{\text {fin }}$ results in rise in fan power consumption, there is a marked increase in net power and decreases in thermal resistance. 
The ability of a TEG-micro channel heat sink combined system to produce a net power and enable self-cooling of device is considered. A numerical model has been developed and three-dimensional governing equation for heat transfer and fluid flow are solved using finite volume approach. The results are compared with published experimental data and the model has been to predict the results with good accuracy. Detailed results for pressure drop, temperature and velocity field has been obtained.

The net power from the system for different set of combination of heat flux and coolant flow rate is considered, it has been found out there is a certain range of coolant flow rate and heat flux combination which could result in net positive power and optimum junction temperature at the heat generating device. Due to the low thermal resistance of microchannel heat sink system, it has been found out that self-cooling could be achieved with lower flow rates (less pumping power) for certain heat flux rate. Thus, for certain applications, it could be feasible to cool the devices without needing any external power systems if the systems are designed in such a way that the minimum flow rate is obtained that controls the temperature as well as results in lower pumping power.

A dynamic model has been developed that could be used to study the performance of TEG based system. The aim of model is to construct a fast and accurate dynamic model which requires less computational resources in terms of size and running time. Thus, the model is constructed by systematically 
representing each part thermal elements and connecting the parts using the electrical analogy. To increase the accuracy of the model, test of Biot number could be made to determine the number of sub-parts needed to represent a physical object. The methodology also employs thermal and electrical model for TEG module which are coupled with temperature and current signals. The cold side heat sink is also coupled with the electrical model.

The model could be implemented in any appropriate software like MATLAB/Simulink, Dymola or SPICE. In this study, Simscape toolbox in MATLAB/Simulink is used to demonstrate how the model works. The results from the model been compared with experimental data and it observed that the model can represent the experimental data to very good accuracy. In addition, the model runs in few seconds and requires minimal computational resources.

\subsection{Future work}

The following are some suggestions for future work in this field:

1) One of the bottlenecks for self-cooling application is the efficiency of TEG modules. If the efficiency of the TEG modules to produce more voltage and electric power is improved, self-cooling application could be widely applicable. Thus, a study which improves the efficiency of the TEG modules especially in terms of new materials would be important. 
2) The study could also include the application for self-cooling to new methods of cooling systems. Liquid cooling in microchannels could be extended to cases of two phase liquids, nano-fluid and liquid metals. However, this needs to be studied in detail as the effects on an increased pumping power, corrosion and other long term effects should be considered.

3) Miniaturization of TEG modules could also aid in better integration of modules into smaller heating devices. Therefore, further investigation on the application of micro-TEG modules for self-cooling application could be done.

4) It is also crucial to study how self-cooling systems could be designed for high heat flux applications. The cooling methods applicable to high heat flux would require more power for the fluid mover, thus it is vital to look at ways to increase the put from TEG modules that enables the generation of adequate power to run the fluid movers.

5) The numerical methodology could be expanded for different cooling methods to increase its applicability 


\section{REFERENCES}

[1] A. Date, A. Date, C. Dixon, A. Akbarzadeh, Progress of thermoelectric power generation systems: Prospect for small to medium scale power generation, Renew. Sustain. Energy Rev. 33 (2014) 371-381. doi:10.1016/j.rser.2014.01.081.

[2] X. Gou, H. Xiao, S. Yang, Modeling, experimental study and optimization on low-temperature waste heat thermoelectric generator system, Appl. Energy. 87 (2010) 3131-3136. doi:10.1016/j.apenergy.2010.02.013.

[3] X. Liu, C. Li, Y.D. Deng, C.Q. Su, An energy-harvesting system using thermoelectric power generation for automotive application, Int. J. Electr. Power Energy Syst. 67 (2015) 510-516. doi:10.1016/j.ijepes.2014.12.045.

[4] N.M. Khattab, E.T. El Shenawy, Optimal operation of thermoelectric cooler driven by solar thermoelectric generator, Energy Convers. Manag. 47 (2006) 407-426. doi:10.1016/j.enconman.2005.04.011.

[5] N. Zhu, T. Matsuura, R. Suzuki, T. Tsuchiya, Development of a Small Solar Power Generation System based on Thermoelectric Generator, 2013 Int. Conf. Altern. Energy Dev. Ctries. Emerg. Econ. 2013 AEDCEE. 52 (2014) 651-658. doi:10.1016/j.egypro.2014.07.121.

[6] Y. Shi, Y. Wang, Y. Deng, H. Gao, Z. Lin, W. Zhu, et al., A novel selfpowered wireless temperature sensor based on thermoelectric generators, Energy Convers. Manag. 80 (2014) 110-116. doi:10.1016/j.enconman.2014.01.010.

[7] D. Champier, J.P. Bédécarrats, T. Kousksou, M. Rivaletto, F. Strub, P. Pignolet, Study of a TE (thermoelectric) generator incorporated in a multifunction wood stove, Energy. 36 (2011) 1518-1526. doi:10.1016/j.energy.2011.01.012.

[8] J.-P. Fleurial, P. Gogna, B.C. Li, S. Firdosy, B.J. Chen, C.-K. Huang, et al., Waste Heat Recovery Opportunities for Thermoelectric Generators, (2009). 
[9] J.P. Fleurial, A. Borshchevsky, T. Caillat, R. Ewell, New materials and devices for thermoelectric applications, in: Energy Convers. Eng. Conf. 1997 IECEC-97 Proc. 32nd Intersoc., 1997: pp. 1080-1085 vol.2. doi:10.1109/IECEC.1997.661920.

[10] T.M. Tritt, Thermoelectric Phenomena, Materials, and Applications, Annu. Rev. Mater. Res. 41 (2011) 433-448.

[11] R.D. Abelson, Thermoelectrics Handbook, CRC Press, Boca Raton, FL, 2006.

[12] S. LeBlanc, Thermoelectric generators: Linking material properties and systems engineering for waste heat recovery applications, Sustain. Mater. Technol. 1-2 (2014) 26-35. doi:10.1016/j.susmat.2014.11.002.

[13] G. Crabtree, N. Lewis, Solar energy conversion, Physictoday. (2007) 37. doi:http://dx.doi.org/10.1063/1.2718755.

[14] L.E. Bell, Cooling, heating, generating power, and recovering waste heat with thermoelectric systems., Science. 321 (2008) 1457-1461. doi:10.1126/science.1158899.

[15] J. LaGrandeur, Worldwide funding of Thermoelectrics, (2009).

[16] J. Fairbanks, Automotive Thermoelectric Generators and HVAC, (2013).

[17] T. Hendricks, N. Karri, T. Hogan, C. Cauchy, New Perspectives in Thermoelectric Energy Recovery System Design Optimization, J. Electron. Mater. 42 (2013) 1725-1736. doi:10.1007/s11664-012-2406-x.

[18] X. Liu, Y.D. Deng, K. Zhang, M. Xu, Y. Xu, C.Q. Su, Experiments and simulations on heat exchangers in thermoelectric generator for automotive application, Appl. Therm. Eng. 71 (2014) 364-370. doi:10.1016/j.applthermaleng.2014.07.022. 
[19] C.-T. Hsu, G.-Y. Huang, H.-S. Chu, B. Yu, D.-J. Yao, Experiments and simulations on low-temperature waste heat harvesting system by thermoelectric power generators, Appl. Energy. 88 (2011) 1291-1297. doi:10.1016/j.apenergy.2010.10.005.

[20] T. Kuroki, K. Kabeya, K. Makino, T. Kajihara, H. Kaibe, H. Hachiuma, et al., Thermoelectric Generation Using Waste Heat in Steel Works, J. Electron. Mater. 43 (2014) 2405-2410. doi:10.1007/s11664-014-3094-5.

[21] Y. Deng, W. Zhu, Y. Wang, Y. Shi, Enhanced performance of solar-driven photovoltaic-thermoelectric hybrid system in an integrated design, Sol. Energy. 88 (2013) 182-191. doi:10.1016/j.solener.2012.12.002.

[22] W. He, Y. Su, Y.Q. Wang, S.B. Riffat, J. Ji, A study on incorporation of thermoelectric modules with evacuated-tube heat-pipe solar collectors, Renew. Energy. 37 (2012) 142-149. doi:10.1016/j.renene.2011.06.002.

[23] H.A. Sodano, G.E. Simmers, R. Dereux, D.J. Inman, Recharging Batteries using Energy Harvested from Thermal Gradients, J. Intell. Mater. Syst. Struct. 18 (2007) 3-10. doi:10.1177/1045389X06063906.

[24] G. Chen, Theoretical efficiency of solar thermoelectric energy generators, J. Appl. Phys. 109 (2011) 104908.

[25] A. Muhtaroglu, A. Yokochi, A. von Jouanne, Integration of thermoelectrics and photovoltaics as auxiliary power sources in mobile computing applications, J. Power Sources. 177 (2008) 239-246. doi:10.1016/j.jpowsour.2007.11.016.

[26] K.J. Kim, F. Cottone, S. Goyal, J. Punch, Energy scavenging for energy efficiency in networks and applications, Bell Labs Tech. J. 15 (2010) 7-29. doi:10.1002/bltj.20438.

[27] T.M. Tritt, H. Böttner, L. Chen, Thermoelectrics: Direct Solar Thermal Energy Conversion, MRS Bull. 33 (2008) 366-368. doi:10.1557/mrs2008.73. 
[28] Thermoelectric Generators for Cathodic Protection, (n.d.). http://www.farwestcorrosion.com/fwst/dcpower/global01.htm.

[29] J.P. Carmo, L.M. Goncalves, J.H. Correia, Thermoelectric Microconverter for Energy Harvesting Systems, Ind. Electron. IEEE Trans. On. 57 (2010) 861-867. doi:10.1109/TIE.2009.2034686.

[30] Z. Wang, V. Leonov, P. Fiorini, C. Van Hoof, Realization of a wearable miniaturized thermoelectric generator for human body applications, EUROSENSORS XXII 2008. $156 \quad$ (2009) 95-102. doi:10.1016/j.sna.2009.02.028.

[31] T. Torfs, V. Leonov, R.F. Yazicioglu, P. Merken, C. Van Hoof, R.J.M. Vullers, et al., Wearable Autonomous Wireless Electro-encephalography System Fully Powered by Human Body Heat, in: Sens. 2008 IEEE, 2008: pp. 1269-1272. doi:10.1109/ICSENS.2008.4716675.

[32] V. Leonov, B. Gyselinckx, C. van Hoof, T. Torfs, R.F. Yazicioglu, R.J.M. Vullers, et al., Wearable self-powered wireless devices with thermoelectric energy scavengers, in: Integr. Issues Miniaturized Syst. - MOMS MOEMS ICS Electron. Compon. SSI 2008 2nd Eur. Conf. Exhib. On, 2008: pp. 1-8.

[33] T. Torfs, V. Leonov, C. Van Hoof, B. Gyselinckx, Body-Heat Powered Autonomous Pulse Oximeter, in: Sens. 2006 5th IEEE Conf. On, 2006: pp. 427-430. doi:10.1109/ICSENS.2007.355497.

[34] J.A. Paradiso, T. Starner, Energy scavenging for mobile and wireless electronics, Pervasive Comput. IEEE. 4 (2005) 18-27. doi:10.1109/MPRV.2005.9.

[35] K. Yazawa, G.L. Solbrekken, A. Bar-Cohen, Thermoelectric-powered convective cooling of microprocessors, Adv. Packag. IEEE Trans. On. 28 (2005) 231-239. doi:10.1109/TADVP.2005.846854.

[36] G.L. Solbrekken, K. Yazawa, A. Bar-Cohen, Heat driven cooling of portable electronics using thermoelectric technology, Adv. Packag. IEEE Trans. On. 31 (2008) 429-437. doi:10.1109/TADVP.2008.920356. 
[37] A. Martínez, D. Astrain, A. Rodríguez, Experimental and analytical study on thermoelectric self cooling of devices, PRES 2010. 36 (2011) 5250-5260. doi:10.1016/j.energy.2011.06.029.

[38] Yu Zhou, S. Paul, S. Bhunia, Harvesting Wasted Heat in a Microprocessor Using Thermoelectric Generators: Modeling, Analysis and Measurement, in: 2008: pp. 98-103. doi:10.1109/DATE.2008.4484669.

[39] C.A. Gould, N.Y.A. Shammas, S. Grainger, I. Taylor, Thermoelectric cooling of microelectronic circuits and waste heat electrical power generation in a desktop personal computer, Microtechnology Therm. Probl. Electron. 176 (2011) 316-325. doi:10.1016/j.mseb.2010.09.010.

[40] Robel Kiflemariam, Cheng-Xian Lin, Rouhollah Moosavi, Numerical simulation, parametric study and optimization of thermoelectric generators for self-cooling of devices, in: 11th AIAAASME Jt. Thermophys. Heat Transf. Conf., American Institute of Aeronautics and Astronautics, 2014. http://dx.doi.org/10.2514/6.2014-3123 (accessed July 20, 2014).

[41] R. Kiflemariam, C.-X. Lin, Numerical Simulation and Parametric Study of Heat-Driven Self-Cooling of Electronic Devices, J. Therm. Sci. Eng. Appl. 7 (2015) 011008-011008. doi:10.1115/1.4028906.

[42] R. Kiflemariam, C.-X. Lin, Numerical simulation of integrated liquid cooling and thermoelectric generation for self-cooling of electronic devices, Int. J. Therm. Sci. 94 (2015) 193-203. doi:10.1016/j.ijthermalsci.2015.02.012.

[43] R.Y. Nuwayhid, F. Moukalled, N. Noueihed, On entropy generation in thermoelectric devices, Energy Convers. Manag. 41 (2000) 891-914. doi:10.1016/S0196-8904(99)00164-8.

[44] F.L. Curzon, B. Ahlborn, Efficiency of a Carnot engine at maximum power output, Am. J. Phys. 43 (1975) 22-24.

[45] A. De Vos, Efficiency of some heat engines at maximum-power conditions, Am. J. Phys. 53 (1985) 570-573. 
[46] A. De Vos, P. van der Wel, The efficiency of the conversion of solar energy into wind energy by means of Hadley cells, Theor. Appl. Climatol. 46 (1993) 193-202. doi:10.1007/BF00865706.

[47] R.Y. Nuwayhid, F. Moukalled, The effect of planet thermal conductance on conversion of solar energy into wind energy, Renew. Energy. 4 (1994) 53-58. doi:10.1016/0960-1481(94)90064-7.

[48] S.. Kaushik, S. Kumar, Finite time thermodynamic analysis of endoreversible Stirling heat engine with regenerative losses, Energy. 25 (2000) 989-1003. doi:10.1016/S0360-5442(00)00023-2.

[49] I. Tlili, Finite time thermodynamic evaluation of endoreversible Stirling heat engine at maximum power conditions, Renew. Sustain. Energy Rev. 16 (2012) 2234-2241. doi:10.1016/j.rser.2012.01.022.

[50] L. Chen, F. Sun, C. Wu, Thermo-economics for endoreversible heatengines, Appl. $\quad$ Energy. $81 \quad$ (2005) 388-396. doi:10.1016/j.apenergy.2004.09.008.

[51] Y. Ust, A. Safa, B. Sahin, Ecological performance analysis of an endoreversible regenerative Brayton heat-engine, Appl. Energy. 80 (2005) 247-260. doi:10.1016/j.apenergy.2004.04.009.

[52] O. Bautista, F. Méndez, J.G. Cervantes, An endoreversible three heat source refrigerator with finite heat capacities, Energy Convers. Manag. 44 (2003) 1433-1449. doi:10.1016/S0196-8904(02)00141-3.

[53] J.M. Gordon, The impact of heat transfer mode on the efficiency of heat engines operating at maximum power, in: Ninth Heat Transf., Hemisphere Publishing, Jerusalem, 1990: pp. 329-334.

[54] W.Y. Lee, S.S. Kim, Finite time optimization of a rankine heat engine, Energy Convers. Manag. 33 (1992) 59-67. doi:10.1016/01968904(92)90147-O. 
[55] C. Wu, Power optimization of a finite-time Carnot heat engine, Energy. 13 (1988) 681-687. doi:10.1016/0360-5442(88)90099-0.

[56] S. Lee, S. Song, V. Au, Moran, Constriction/spreading resistance model for electronic packing, in: 1995: pp. 109-206.

[57] P. Teertstra, M.M. Yovanovich, J.R. Culham, T. Lemczyk, Analytical forced convection modeling of plate fin heat sinks, in: Semicond. Therm. Meas. Manag. Symp. 1999 Fifteenth Annu. IEEE, 1999: pp. 34-41. doi:10.1109/STHERM.1999.762426.

[58] J.R. Culham, M.M. Yovanovich, S. Lee, Thermal modeling of isothermal cuboids and rectangular heat sinks cooled by natural convection, Compon. Packag. Manuf. Technol. Part IEEE Trans. On. 18 (1995) 559-566. doi:10.1109/95.465153.

[59] M. Ekpu, R. Bhatti, M.I. Okereke, S. Mallik, K. Otiaba, The effect of thermal constriction on heat management in a microelectronic application, Microelectron. J. 45 (2014) 159-166. doi:10.1016/j.mejo.2013.10.011.

[60] M. Grujicic, C.L. Zhao, E.C. Dusel, The effect of thermal contact resistance on heat management in the electronic packaging, Appl. Surf. Sci. 246 (2005) 290-302. doi:10.1016/j.apsusc.2004.11.030.

[61] S. Song, M.M. Yovanovich, F.O. Goodman, Thermal Gap Conductance of Conforming Surfaces in Contact, J. Heat Transf. 115 (1993) 533-540. doi:10.1115/1.2910719.

[62] W.M. Kays, A.L. London, Compact Heat Exchangers, 2nd ed., McGrawHill, 1984.

[63] D. Copeland, Optimization of parallel plate heatsinks for forced convection, in: Semicond. Therm. Meas. Manag. Symp. 2000 Sixt. Annu. IEEE, 2000: pp. 266-272. doi:10.1109/STHERM.2000.837093.

[64] Specfication of Generating Thermoelectric Modules TGM-287-1.0-1.5, (n.d.). http://kryothermtec.com/assets/dir2attz/ru/TGM-287-1.0-1.5.pdf. 
[65] Fan Specfication document, (n.d.).

[66] H. Coleman, W. Steele, Experimentation, Validation, and Uncertainty Analysis for Engineers, 3rd Edition, Wiley, New York, 2009.

[67] F.M. White, Fluid mechanics, 5th ed, McGraw-Hill, New York, 2003.

[68] Fan Performance Data, (2014). www.sunon.com.

[69] D.M. Rowe, Thermoelectrics Handbook: Macro to Nano, CRC press, Boca Raton, FL, 2005.

[70] M. Chen, L.A. Rosendahl, T. Condra, A three-dimensional numerical model of thermoelectric generators in fluid power systems, Int. J. Heat Mass Transf. 54 (2011) 345-355. doi:10.1016/j.ijheatmasstransfer.2010.08.024.

[71] X. Gou, H. Xiao, S. Yang, Modeling, experimental study and optimization on low-temperature waste heat thermoelectric generator system, Appl. Energy. 87 (2010) 3131-3136. doi:10.1016/j.apenergy.2010.02.013.

[72] X.-D. Wang, Y.-X. Huang, C.-H. Cheng, D. Ta-Wei Lin, C.-H. Kang, A three-dimensional numerical modeling of thermoelectric device with consideration of coupling of temperature field and electric potential field, AsiaPac. Forum Renew. Energy 2011. 47 (2012) 488-497. doi:10.1016/j.energy.2012.09.019.

[73] D.N. Kossyvakis, C.G. Vossou, C.G. Provatidis, E.V. Hristoforou, Computational analysis and performance optimization of a solar thermoelectric generator, Renew. Energy. 81 (2015) 150-161. doi:10.1016/j.renene.2015.03.026.

[74] S. Kim, Analysis and modeling of effective temperature differences and electrical parameters of thermoelectric generators, Spec. Issue Adv. Sustain. Biofuel Prod. Use - XIX Int. Symp. Alcohol Fuels - ISAF. 102 (2013) 14581463. doi:10.1016/j.apenergy.2012.09.006. 
[75] G. Wu, X. Yu, A holistic 3D finite element simulation model for thermoelectric power generator element, Energy Convers. Manag. 86 (2014) 99-110. doi:10.1016/j.enconman.2014.04.040.

[76] M. Zhou, Y. He, Y. Chen, A heat transfer numerical model for thermoelectric generator with cylindrical shell and straight fins under steadystate conditions, Appl. Therm. Eng. 68 (2014) 80-91. doi:10.1016/j.applthermaleng.2014.04.018.

[77] A. Martínez, D. Astrain, A. Rodríguez, Dynamic model for simulation of thermoelectric self cooling applications, Energy. 55 (2013) 1114-1126. doi:10.1016/j.energy.2013.03.093.

[78] A. Martínez, D. Astrain, A. Rodríguez, Experimental and analytical study on thermoelectric self cooling of devices, PRES 2010. 36 (2011) 5250-5260. doi:10.1016/j.energy.2011.06.029.

[79] COMSOL Multiphysics Version 4.2, (2011).

[80] ANSYS Fluent theory and user guide, Release 14, (2011.).

[81] M. Chen, G.J. Snyder, Analytical and numerical parameter extraction for compact modeling of thermoelectric coolers, Int. J. Heat Mass Transf. 60 (2013) 689-699. doi:10.1016/j.ijheatmasstransfer.2013.01.020.

[82] F. James, A. William, Material science and engineering handbook, CRC press, 2001.

[83] B. Agostini, M. Fabbri, J.E. Park, L. Wojtan, J.R. Thome, B. Michel, State of the Art of High Heat Flux Cooling Technologies, Heat Transf. Eng. 28 (2007) 258-281. doi:10.1080/01457630601117799.

[84] D.B. Tuckerman, R.F.W. Pease, High-performance heat sinking for VLSI, Electron Device Lett. IEEE. 2 (1981) 126-129. doi:10.1109/EDL.1981.25367. 
[85] I. Hassan, P. Phutthavong, M. Abdelgawad, MICROCHANNEL HEAT SINKS: AN OVERVIEW OF THE STATE-OF-THE-ART, Microscale Thermophys. Eng. 8 (2004) 183-205. doi:10.1080/10893950490477338.

[86] S.G. Kandlikar, High Flux Heat Removal with Microchannels-A Roadmap of Challenges and Opportunities, Heat Transf. Eng. 26 (2005) 5-14. doi:10.1080/01457630591003655.

[87] A. Rezania, L.A. Rosendahl, Evaluating Thermoelectric Power Generation Device Performance Using a Rectangular Microchannel Heat Sink, J. Electron. Mater. 40 (2011) 481-488. doi:10.1007/s11664-011-1622-0.

[88] N. Wojtas, L. Rüthemann, W. Glatz, C. Hierold, Optimized thermal coupling of micro thermoelectric generators for improved output performance, Renew. Energy. 60 (2013) 746-753. doi:10.1016/j.renene.2013.06.031.

[89] N. Wojtas, E. Schwyter, W. Glatz, S. Kühne, W. Escher, C. Hierold, Power enhancement of micro thermoelectric generators by microfluidic heat transfer packaging, Sel. Pap. 16th Int. Conf. Solid-State Sens. Actuators Microsyst. 188 (2012) 389-395. doi:10.1016/j.sna.2011.12.043.

[90] A. Rezania, L.A. Rosendahl, S.J. Andreasen, Experimental investigation of thermoelectric power generation versus coolant pumping power in a microchannel heat sink, Int. Commun. Heat Mass Transf. 39 (2012) 10541058. doi:10.1016/j.icheatmasstransfer.2012.07.010.

[91] G.E. Moore, Progress in digital integrated electronics, in: Electron Devices Meet. 1975 Int., 1975: pp. 11-13.

[92] R. Chein, J. Chen, Numerical study of the inlet/outlet arrangement effect on microchannel heat sink performance, Int. J. Therm. Sci. 48 (2009) 16271638. doi:10.1016/j.jithermalsci.2008.12.019.

[93] A. Rezania, L.A. Rosendahl, Thermal effect of a thermoelectric generator on parallel microchannel heat sink, 7th Bienn. Int. Workshop "Advances Energy Stud. 37 (2012) 220-227. doi:10.1016/j.energy.2011.11.043. 
[94] TCS Micropumps Ltd, M400 Series - Data Sheet, (2011).

[95] Simscape/Simulink toolbox: User's Guide, (2014). 


\section{APPENDIX A}

A computer code to analyze TEG based heat driven self-cooling system

The code is based on the algorithm developed using finite time thermodynamics principles. The code is written in both Python and MATLAB codes.

Python code:

__author__ = 'robel kiflemariam'

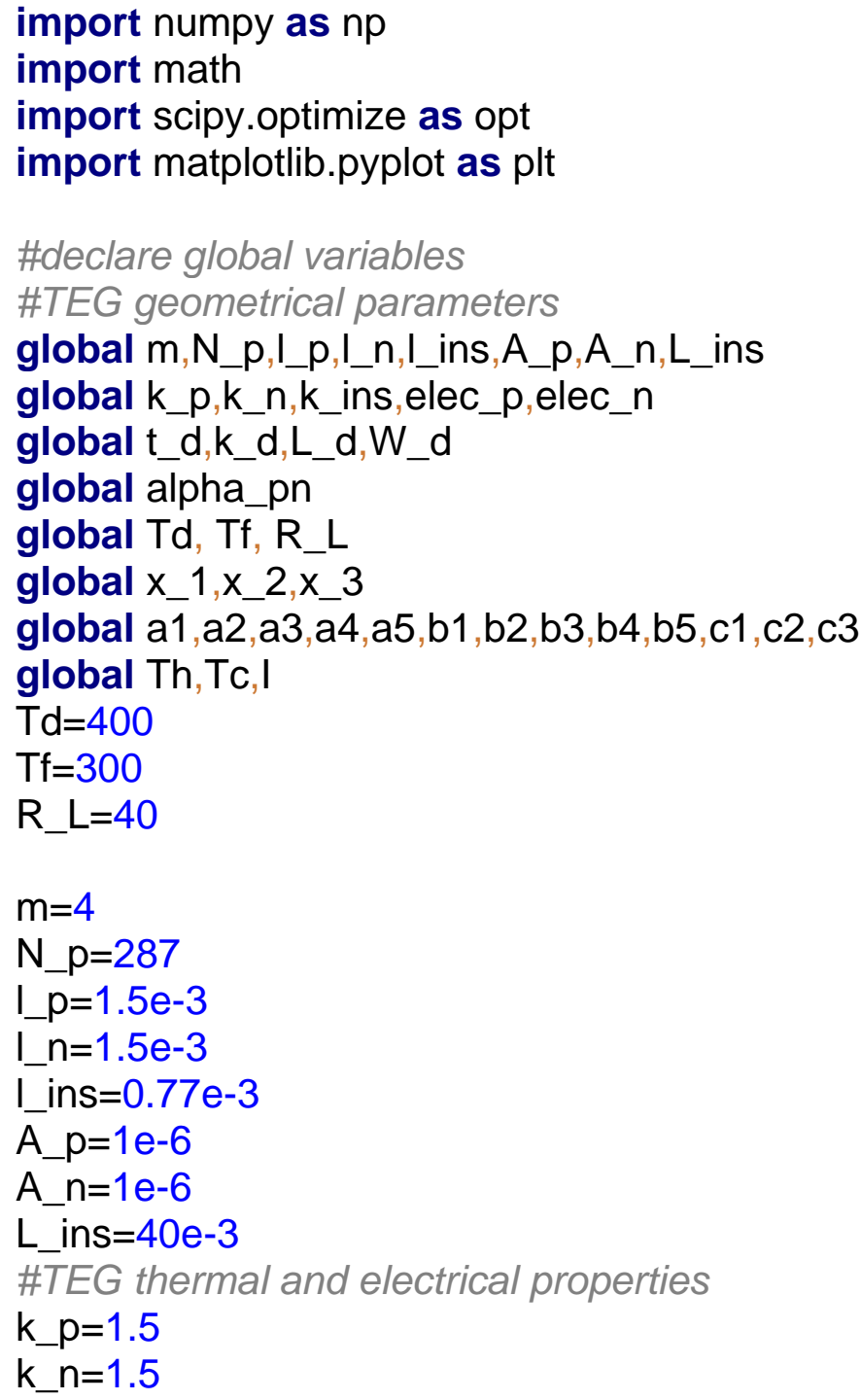




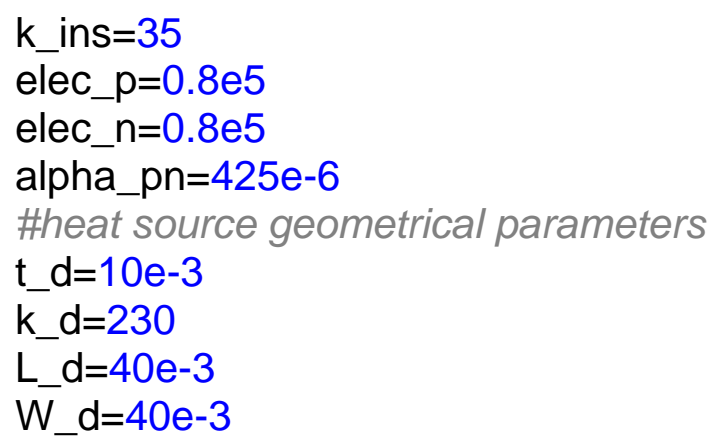

\#Function calculates the conductance of TEG array

def $k$ th_array $\left(m, k \_p, k \_n, k \_\right.$ins, I_p,I_n,I_ins, A_p, A_n,L_ins, N_p):

N_p_a $=m * N \_p$

A_ins_a $=m^{*} \operatorname{pow}\left(L_{-}\right.$ins, 2$)$

$R$ _th_p=I_p/(A_p*k_p)

R_th_n=I_n/(A_n*k_n)

$R$ th_ins $=2 *$ I_ins/(A_ins_a*k_ins)

R_th_pn_array $=\left(R \_\overline{t h} p^{\star} R \_\right.$th $\left.\_n\right) /\left(N \_p \_a *\left(R \_t h \_p+R \_t h \_n\right)\right)$

R_th_TEG_array $=\bar{R}$ _th_pn_array+R_th_ins

K_th_TEG_array_l=pow(R_th_TEG_array, -1$)$

return(K_th_TEG_array_I)

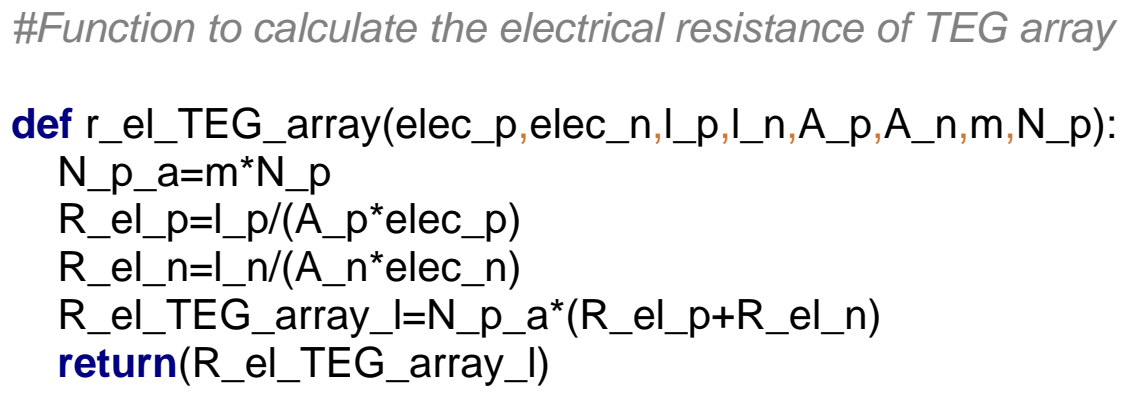




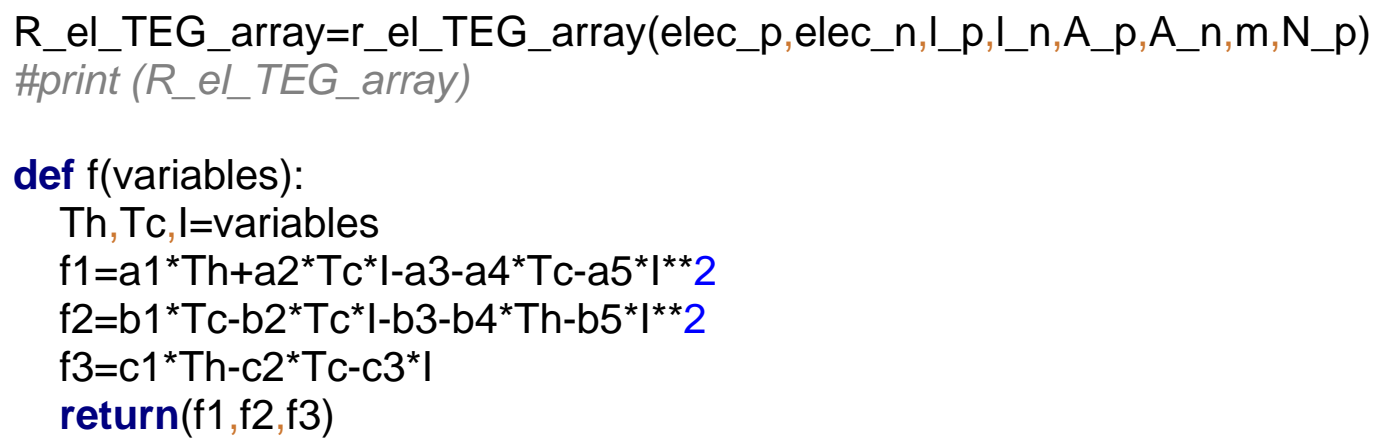

\#Cold side heat sink parameters

global t_b,H_f,t_f,rho_f,mew_f,Cp_f,k_b,k_f,b,W,W_fi,L

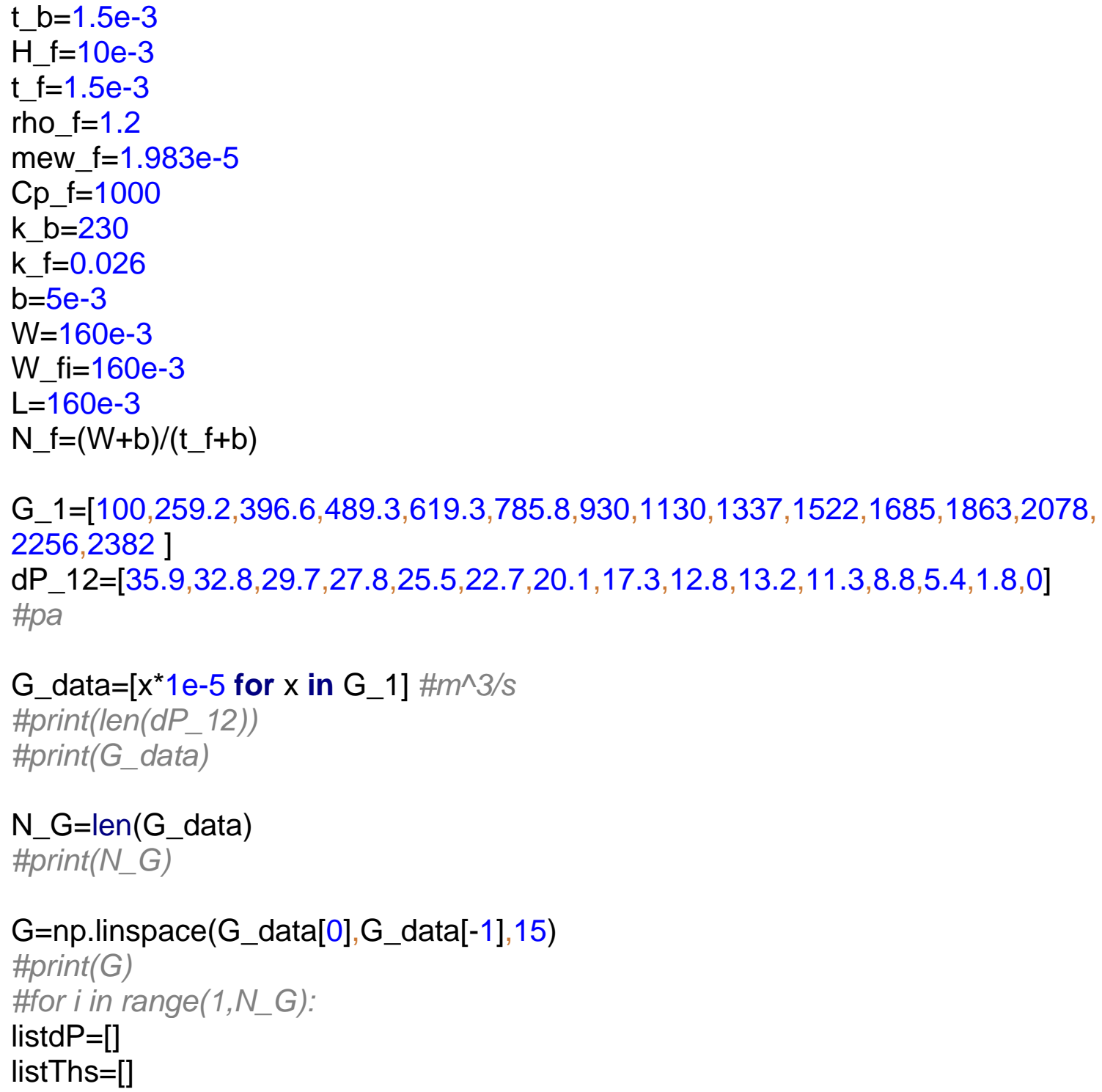




$$
\begin{aligned}
& \text { listTcs }=\square \\
& \text { listl }=[] \\
& \text { listV_oc= } \square \\
& \text { listV }=[] \\
& \text { listqh }=[] \\
& \text { listqc }=[ \\
& \text { listpower }=[] \\
& \text { listEff }=\square \\
& \text { listDT }=[]
\end{aligned}
$$

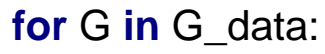

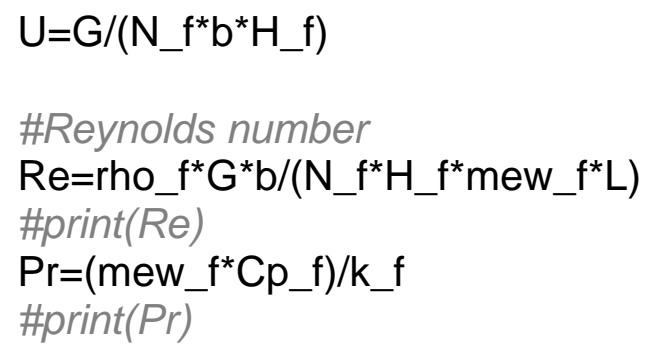

$\mathrm{Nu}=\left(\left(1 / \mathrm{np}\right.\right.$. power $\left.\left(\left(0.5^{\star} \operatorname{Re}^{\star} \mathrm{Pr}\right), 3\right)\right)+\left(1 /\left(0.644^{\star} n p\right.\right.$. sqrt $(\operatorname{Re})^{\star} \mathrm{np}$. power $(\operatorname{Pr}, 0.33)^{\star} \mathrm{np} . \mathrm{sqr}$ $\left.\mathrm{t}(1+3.65 / \mathrm{np} . \mathrm{sqrt}(\mathrm{Re}))))^{\star \star} 3\right)^{\star \star}-0.33$

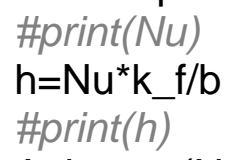

A_2 $=\mathrm{L} * \mathrm{~W}$

A_1 $1=4 *$ L_ins ${ }^{\star *} 2$

gamma $=\left(\left(3.14^{\star \star} 1.5\right) / n p . \operatorname{sqrt}\left(A \_2\right)+(\text { np.sqrt(A_1) })^{\star \star}-1\right)$ omega_1=gamma ${ }^{*} k b^{*} A \_2^{*} R \_c o n v^{*} n p . t a n h\left(g a m m a^{\star} t b\right)$ omega=omega_1/(1+omega_1)

R_sp_b=omega ${ }^{\star}\left(1 /\left(2^{*} k \text { b }{ }^{\star} n p . s q r t\left(A \_1\right)\right)\right)^{\star}\left(\left(1-n p . s q r t\left(A \_1\right) / n p . s q r t\left(A \_2\right)\right)\right)^{\star *} 1.5$ R_sp_a $=\left(\left(n p . s q r t\left(A \_2\right)-n p . s q r t\left(A \_1\right)\right)^{\star}\right.$ omega $) /\left(k \_b{ }^{\star} n p . s q r t\left(3.14^{\star} A \_2^{\star} A \_1\right)\right)$

R_sp=R_sp_b+R_sp_a

R_CX=R_conv+R_sp

$U \bar{A} \_C X=\bar{R} \_C X^{* *}-\overline{1}$ 


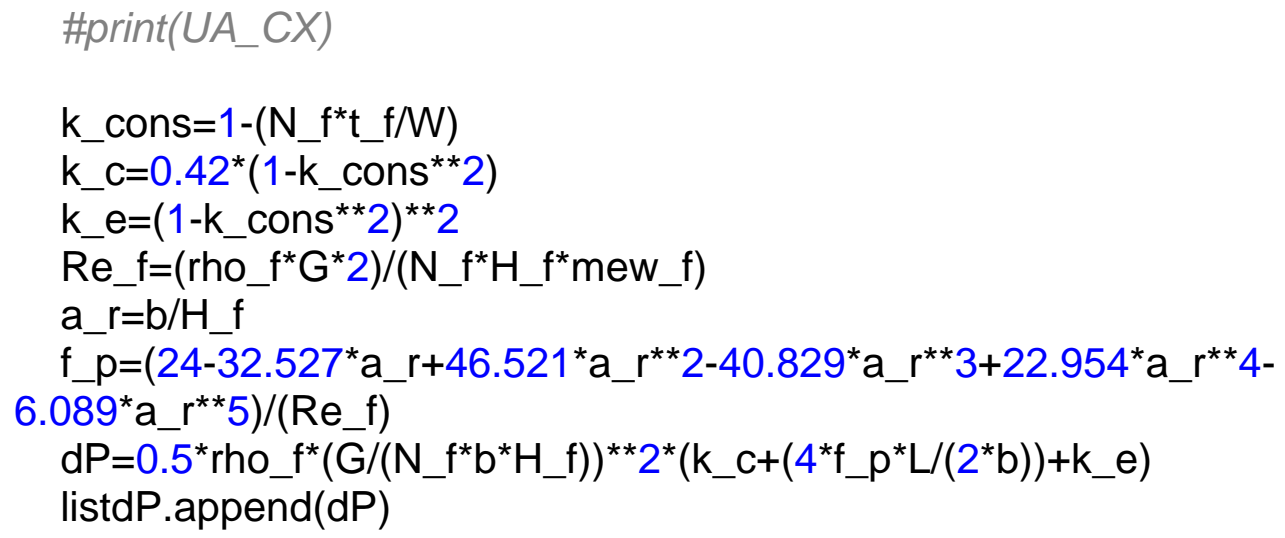

def $f($ variables):

Ths, Tcs, I=variables

f1 $=a 1^{*}$ Ths $+a 2 *$ Ths*|-a3-a4*Tcs-a5*|**2

f2=b1*Tcs-b2*Tcs*|-b3-b4*Ths-b5*|**2

$\mathrm{f} 3=\mathrm{c} 1^{*}$ Ths $-\mathrm{c} 2^{*} \mathrm{Tcs}-\mathrm{c} 3^{\star}$ |

return $(\mathrm{f} 1, \mathrm{f} 2, \mathrm{f} 3)$

Ths, Tcs, I=opt.fsolve $(\mathrm{f},(300,200,0.6))$

listThs.append(Ths)

listTcs.append(Tcs)

listl.append(I)

V_oc=alpha_TEG_array*(Ths-Tcs)

$\mathrm{V}=\mathrm{V}$ oc $/ 2$

q_h $=U A \_H X^{*}(T d-T h s)$ 


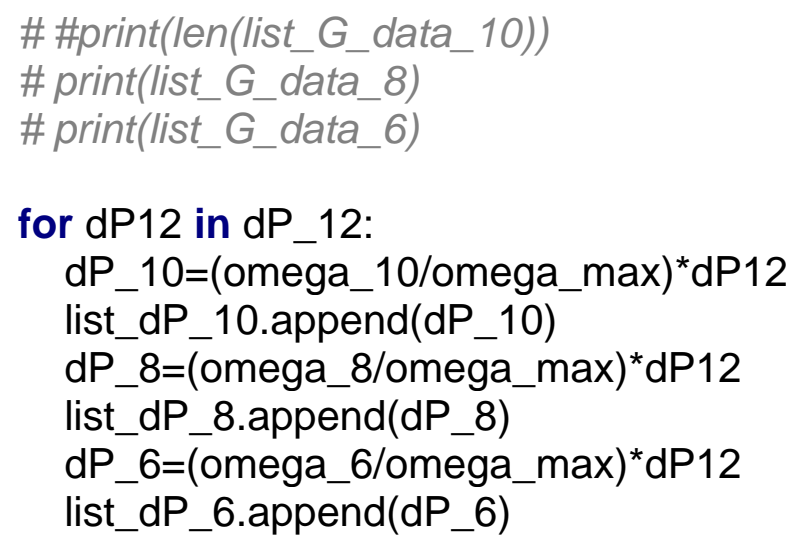

\#fit with a regression line $12 \mathrm{~V}$

G_data_coeff=np.polyfit(G_data,dP_12,4) \#calculates the coeff. of the regression line

poly_dP_12=np.poly1d(G_data_coeff)

y_dP_12=poly_dP_12(G_data)

\#fit with a regression line $10 \mathrm{~V}$

G_data_coeff_10=np.polyfit(G_data,list_dP_10,4) \#calculates the coeff. of the regression line

poly_dP_10=np.poly1d(G_data_coeff_10)

y_dP_10=poly_dP_10(G_data)

\#fit with a regression line $8 \mathrm{~V}$

G_data_coeff_8=np.polyfit(G_data,list_dP_8,4) \#calculates the coeff. of the regression line

poly_dP_8=np.poly1d(G_data_coeff_8)

y_dP_8=poly_dP_8(G_data)

\#fit with a regression line $6 \mathrm{~V}$

G_data_coeff_6=np.polyfit(G_data,list_dP_6,4) \#calculates the coeff. of the regression line

poly_dP_6=np.poly1d(G_data_coeff_6)

y_dP_6=poly_dP_6(G_data)

\#

coeff_sys=np.polyfit(G_data,listdP,4) \#calculates the coeff. of the regression line poly_dP_sys=np.poly1d(coeff_sys)

y_dP_sys=poly_dP_sys(G_data)

\#plt.plot(G_data,y_dP_sys, 'o')

\#print(poly_dP_sys)

\#print(poly_dP_12) 


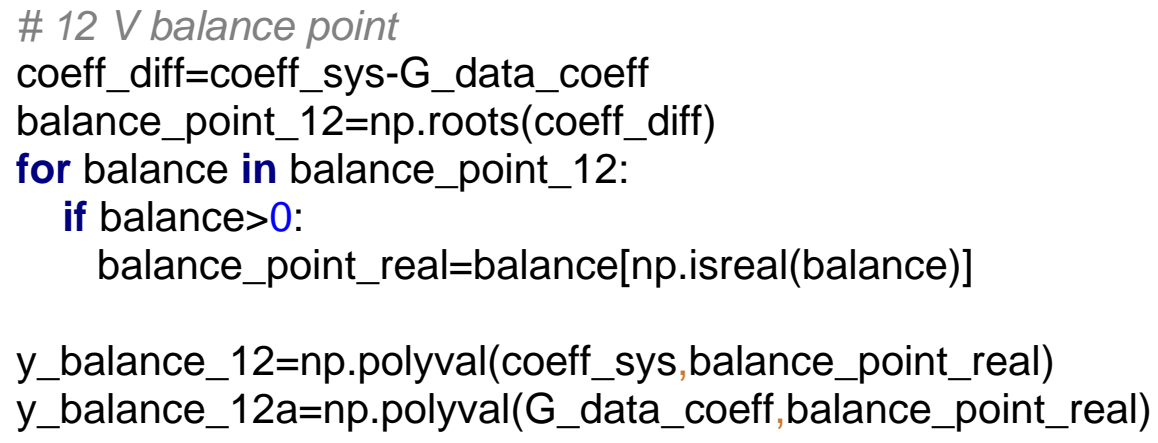

\section{\#10 V balance point}

coeff_diff_10=coeff_sys-G_data_coeff_10 balance_point_10=np.roots(coeff_diff_10)

for balance in balance_point_10:

if balance $>0$ :

balance_point_real_10=balance[np.isreal(balance)]

y_balance_10=np.polyval(coeff_sys,balance_point_real_10)

y_balance_10a=np.polyval(G_data_coeff, balance_point_real)

\section{\#8 $\vee$ balance point}

coeff_diff_8=coeff_sys-G_data_coeff_8 balance_point_8=np.roots(coeff_diff_- 8 )

for balance in balance_point_8:

if balance $>0$ :

balance_point_real_8=balance[np.isreal(balance)]

y_balance_8=np.polyval(coeff_sys,balance_point_real_8)

y_balance_8a=np.polyval(G_data_coeff,balance_point_real)

\section{\#6V balance point}

coeff_diff_6=coeff_sys-G_data_coeff_6 balance_point_6=np.roots(coeff_diff_- 6 )

for balance in balance_point_6:

if balance $>0$ :

balance_point_real_6=balance[np.isreal(balance)]

y_balance_6=np.polyval(coeff_sys,balance_point_real_6)

y_balance_6a=np.polyval(G_data_coeff,balance_point_real_6) 


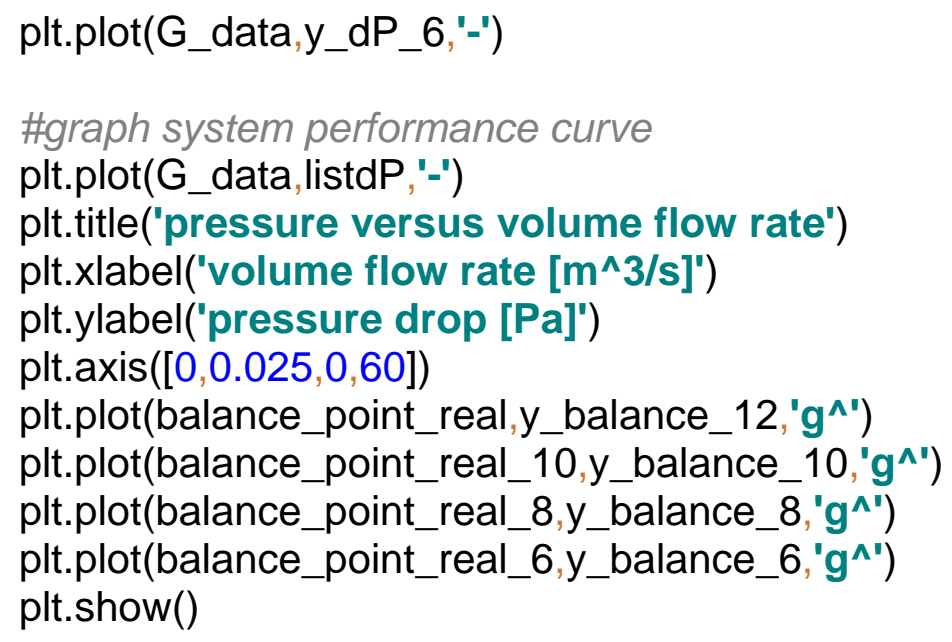




\section{APPENDIX B}

//C based program for User defined functions (UDF)//

//implemented inside ANSYS Fluent//

\#include"udf.h"
real Current;
real Current_flux_h;
real Current_flux_c;

DEFINE_ADJUST(average_temp, domain)

\{

real tavg_T;

real tavg_B;

real area_tot_h;

real area_tot_c;

real tavg;

real temp;

real area;

int ID T;

int ID_B;

real V_OC;

/*FILE *fp;*/

Thread *t, *t1;

face_t $f$;

Domain *d;

$\mathrm{d}=$ Get_Domain(1);

ID_T $=\overline{20}$;

ID_B $=26$;

$\mathrm{t}=$ Lookup_Thread(d,ID_T);

t1=Lookup_Thread(d,ID_B);

tavg_T=0;

tavg_B $=0$;

area $=0$;

area_tot_h=0;

area_tot_c $=0$;

$\mathrm{V} \_\mathrm{OC}=0$; 


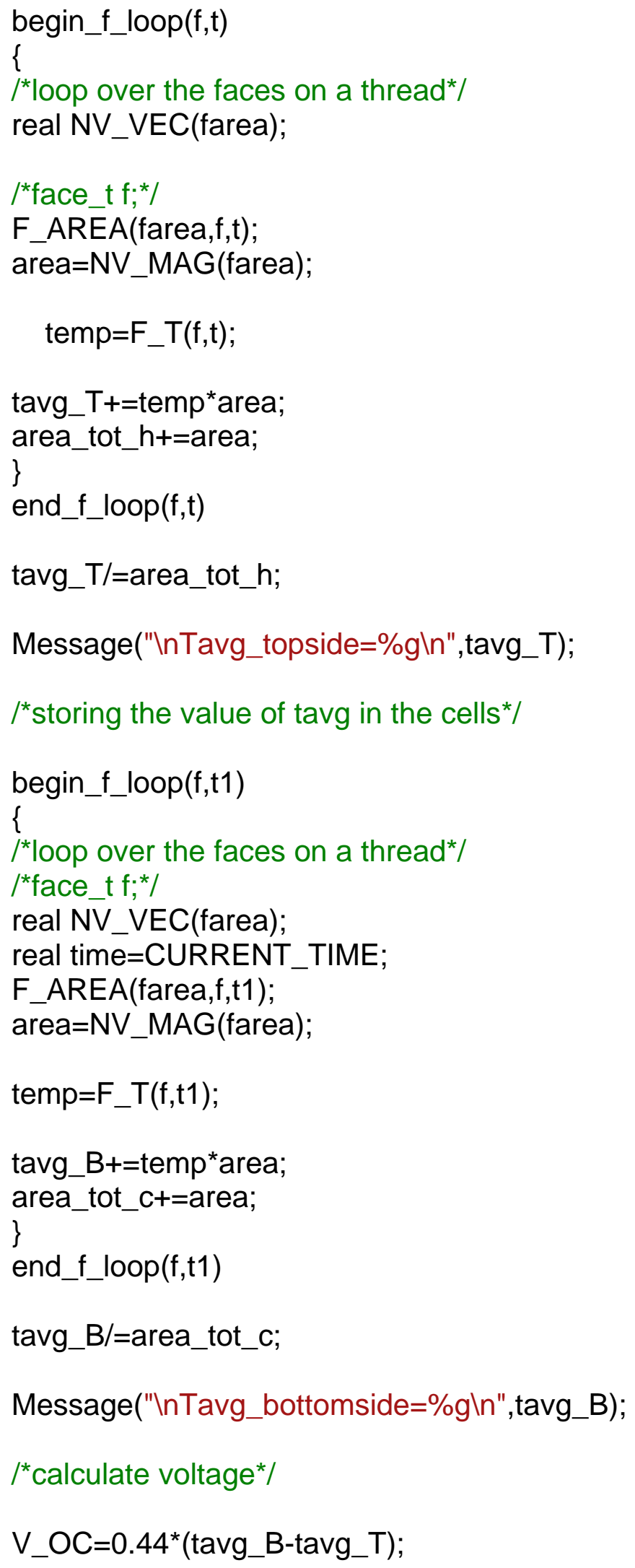




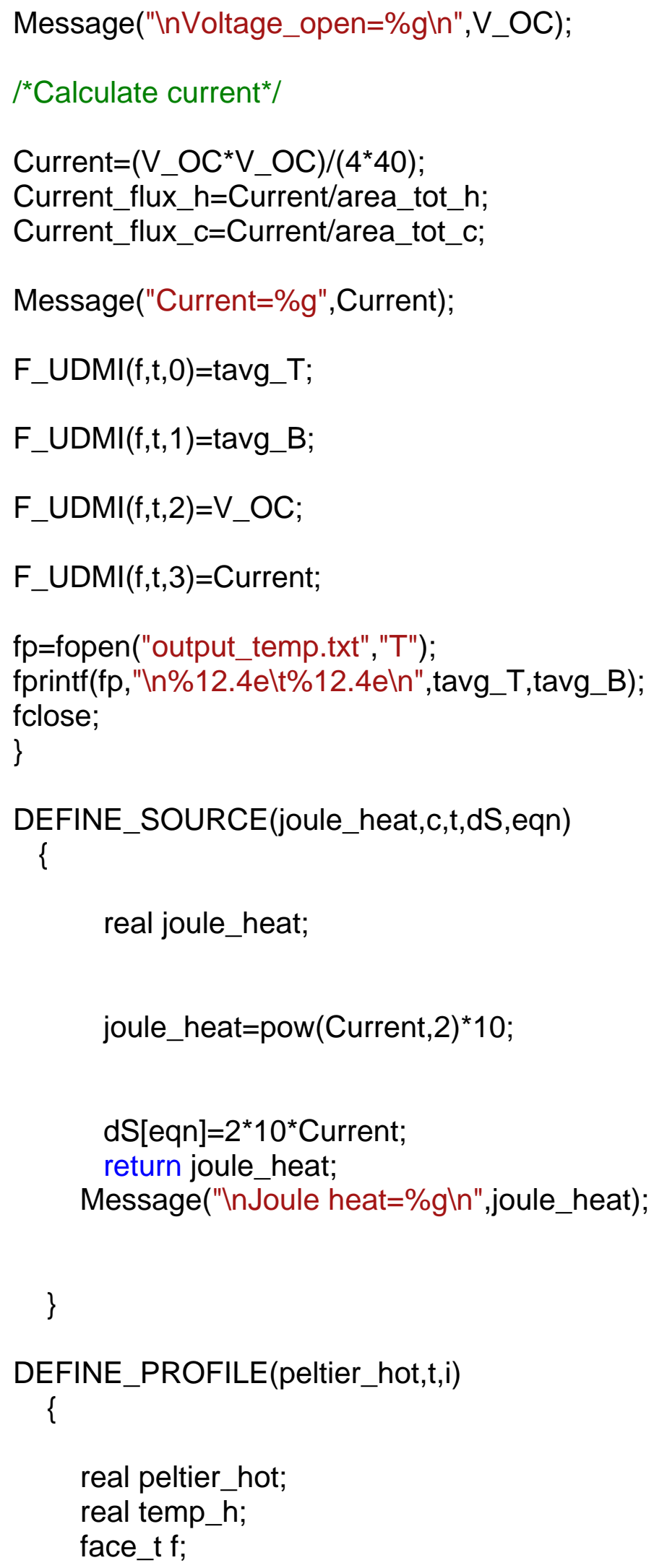


begin_f_loop(f,t)

\{

temp_h=F_T(f,t);

peltier_hot $=0.11^{\star}$ Current_flux_h ${ }^{\star}$ temp_h ;

F_PROFILE $(f, t, i)=$ peltier_hot;

\}

end_f_loop(f,t)

Message("Inpeltier hot $=\% g \mid n "$,peltier_hot);

F_UDMI (f,t,4)=peltier_hot;

\}

\section{DEFINE_PROFILE(peltier_cold,t,i)}

\{

real peltier_cold;

real temp_c;

face_t f;

begin_f_loop(f,t)

\{

temp_c $=F \_T(f, t)$;

peltier_cold $=0.11^{\star}$ Current_flux_c*temp_c;

F_PROFILE $(f, t, i)=$ peltier_cold;

\}

end_f_loop $(\mathrm{f}, \mathrm{t})$

Message("Inpeltier cold =\%gln",peltier_cold);

F_UDMI $(f, t, 5)=$ peltier_cold;

\}

DEFINE_PROFILE(fan_curve,t,i)

\{ 


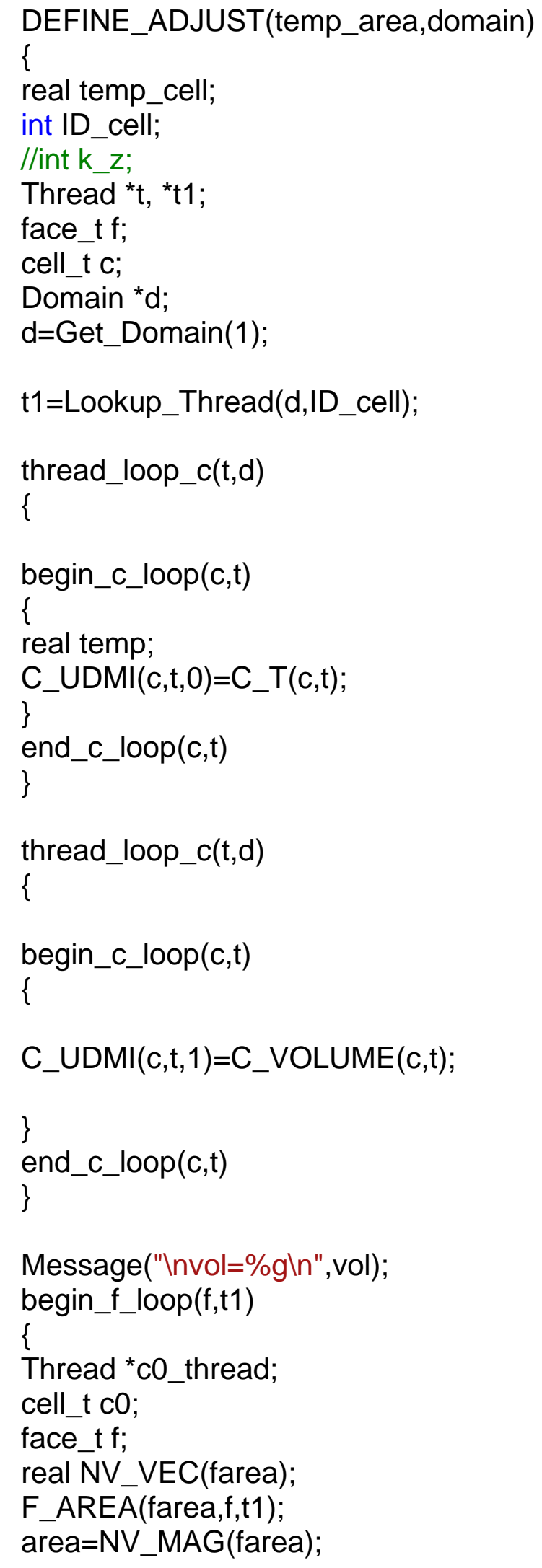




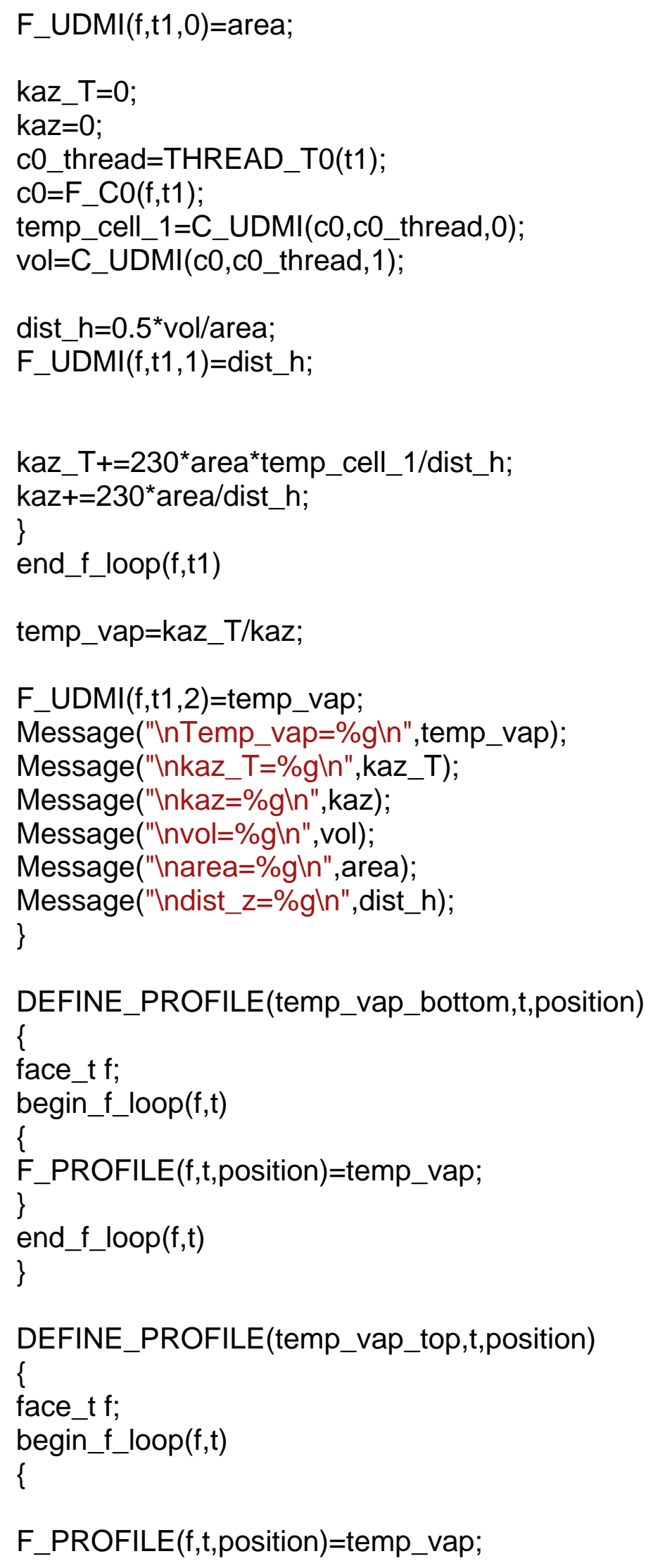


\}

end_f_loop $(f, t)$

\} 
VITA

ROBEL KIFLEMARIAM

1999-2003

BSc. Mechanical Engineering

University of Asmara

Asmara, Eritrea

2011-2012

MSc. Mechanical Engineering

Florida International University

Miami, Florida

2012-2015

Doctoral Candidate

Florida international University

Miami, Florida

\section{PUBLICATIONS AND PRESENTATIONS}

R. Kiflemariam, C.-X. Lin, Numerical simulation of integrated liquid cooling and thermoelectric generation for self-cooling of electronic devices, Int. J. Therm. Sci. 94 (2015) 193-203.

R. Kiflemariam, C.-X. Lin, Numerical Simulation and Parametric Study of HeatDriven Self-Cooling of Electronic Devices, J. Therm. Sci. Eng. Appl. 7 (2015) 011008-011008.

R. Kiflemariam, C.-X. Lin, Numerical simulation on thermoelectric generator based self-cooling application, submitted to Int. J. Therm. Sci.

R. Kiflemariam, C.-X. Lin, R. Mossavi, Experimental investigation on thermoelectric generator based self-cooling application, submitted to App.Eng.

R. Kiflemariam, C.-X. Lin, Computational Models for performance prediction of a hybrid PV/T module, 1st Thermal and Fluid Engineering Summer Conference, TFESC August 9-12, 2015, New York City, USA. 
R. Moosavi, H. Saffari, C. X. Lin, R. Kiflemariam, CFD Simulation of velocity and void fraction distribution of two-phase flows (air-water) in vertical helical coils, 1stThermal and Fluids Engineering Summer Conference, August 9-12, 2015, New York, USA.

R. Kiflemariam, F. Zevallos, C.-X. Lin, Numerical Simulation and Parametric Analysis of Multistage Ionic Wind Pump for Enhanced Air-Cooling, in: IMECE2014, Montreal, Quebec, Canada, 2014. doi:1115/IMECE2014-38368.

F. Zevallos, C.-X. Lin, R. Kiflemariam, A Computational Model for Performance Prediction of a Hybrid PV/T Module, in: IMECE2014, ASME, Montreal, Quebec, Canada, p. 9.doi:10.1115/IMECE2014-38569.

R. Kiflemariam, C.-X. Lin, R. Moosavi, Numerical simulation, parametric study and optimization of thermoelectric generators for self-cooling of devices, in: 11th AIAAASME Jt. Thermophys. Heat Transf. Conf., American Institute of Aeronautics and Astronautics, 2014.

R. Moosavi, S. A. Gandjalikhan Nasab, R. Kiflemariam, C.-X. Lin, The effect of step length and inclined angle of backward facing step on heat transfer characteristics, in: 11th AIAAASME Jt. Thermophys. Heat Transf. Conf., American Institute of Aeronautics and Astronautics, 2014.

R. Moosavi, H. Saffari, R. Kiflemariam, C.-X. Lin, Length of the Entrance, Fully developed and hydraulic characteristics of bubbly flows in helical coils, in: 11th AIAAASME Jt. Thermophys. Heat Transf. Conf., American Institute of Aeronautics and Astronautics, 2014.

R. Kiflemariam, C.-X. Lin, CFD Modeling of Pressure Coefficients for a Natural Ventilation Study in an Experimental Low Rise Building, in: IMECE2013, ASME, San Diego, California, USA, 2013. 